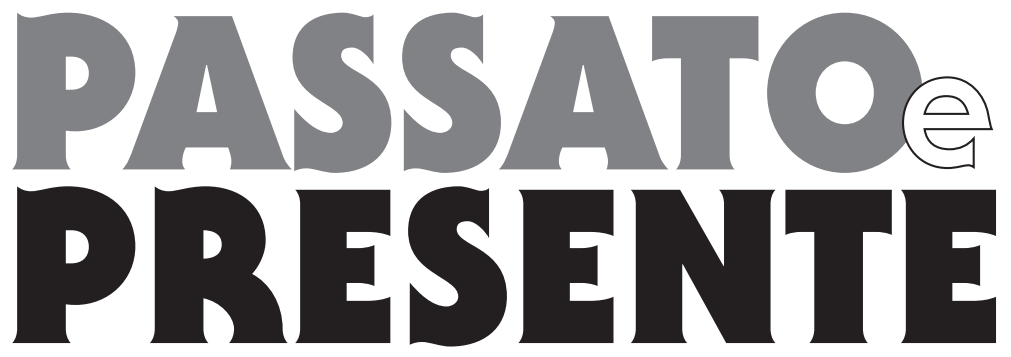

Numero 88

gennaio-aprile 2013

anno XXXI

\title{
FrancoAngel
}




\section{«Passato e presente»}

Rivista di storia contemporanea

Pubblicazione quadrimestrale

\section{Direzione}

Michele Battini, Bruno Bongiovanni, Massimo Cattaneo, Giovanni De Luna, Paul Ginsborg, Giovanni Gozzini, Ilaria Porciani, Anna Rossi-Doria, Gianpasquale Santomassimo,

Luciano Segreto, Francesca Tacchi, Stuart Woolf

Aldo Agosti, Simonetta Soldani coordinatori

Gabriele Turi direttore

Corrispondenti

Roberto Bianchi, Giovanni Borgognone, Carolina Castellano, Valeria Galimi, Wilko Graf von Hardenberg, Stefano Petrungaro, Marcella Simoni, Francesca Zantedeschi, Gilda Zazzara

Redazione

Francesca Tacchi (responsabile), Matteo Mazzoni

Direttore responsabile

Gabriele Turi

Corrispondenza e libri vanno inviati a:

Gabriele Turi - "Passato e presente" - Dipartimento di studi storici e geografici, via S. Gallo 10, 50129 Firenze

pep@unifi.it

http://www.francoangeli.it

Tutti i testi presentati alla rivista sono sottoposti a peer review da parte di referees. Gli articoli pubblicati sono presenti nei principali registri di catalogazione.

Amministrazione, distribuzione, abbonamenti:

FrancoAngeli srl, viale Monza 106, 20127 Milano, tel. 02/2837141

Ufficio abbonamenti: fax 02/2895762

e-mail: riviste@francoangeli.it - http://www.francoangeli.it

Abbonamenti: Per conoscere il canone d'abbonamento corrente, consultare il nostro sito (www.francoangeli.it), cliccando sul bottone "Riviste", oppure telefonare al nostro Ufficio Riviste (02/2837141) o, ancora, inviare una e-mail (riviste@francoangeli.it) indicando chiaramente il nome della rivista

Il pagamento può essere effettuato tramite:

versamento sul c.c.p. n. 17562208 intestato a FrancoAngeli srl, Milano, oppure bonifico intestato a FrancoAngeli srl, Milano c/o Intesa Sanpaolo IT32D0306901601100000061992

L'abbonamento verrà attivato non appena giunta la notifica dell'avvenuto pagamento del canone

Autorizzazione del Tribunale di Firenze n. 3040 del 21 maggio 1982 - quadrimestrale - Poste Italiane spa - Sped. in Abb. Post. - D.L. 353/2003 (conv. in L. 27/02/2004 n. 46) art. 1, comma 1, DCB Milano Copyright 2013 by FrancoAngeli srl - Stampa: Tipomonza, via Merano 18, Milano

I quadrimestre 2013 - Finito di stampare nel febbraio 2013 


\title{
Sommario
}

\author{
Editoriale
}

5

Eric Hobsbawm, un maestro e un amico

Aldo Agosti

Discussioni

13 Volksgemeinschaft tra storiografia e memoria interventi di Michael Wildt, Hans Mommsen, Ulrich Herbert (a cura di Paolo Fonzi)

\section{SAGGi}

$37 \quad$ Combattenti solitari.

Lavoratori tedeschi e ungheresi in epoca postcomunista Eszter Bartha

57 Città nuove, colonizzazione e impero. Il caso di Fertilia Marcel Farinelli

\section{STORICI CONTEMPORANEI}

83 Ricerca storica e cittadinanza attiva: intervista a Eric Foner Daria Frezza e Alessandra Lorini

\section{MASS MEDIA}

111 "Spara e prega!".

Il cinema israeliano a trent'anni dalla guerra del Libano Marcella Simoni

\section{FONTI}

$131 \quad$ Alla Moneda con Salvador Allende Gennaro Carotenuto

\section{RECENSIONI}

153 Né Est né Ovest: verso una nuova storia del '900 europeo? Marco Bresciani

\section{SCHEDE}

$163 \quad$ Fumetti nella storia, storia nei fumetti a cura di Roberto Bianchi 


\section{Contents}

\section{EDITORIAL}

Eric Hobsbawm, a mentor and a friend

Aldo Agosti

Debates

13 Volksgemeinschaft between historiography and memory Michael Wildt, Hans Mommsen, Ulrich Herbert (edited by Paolo Fonzi)

$37 \quad$ Lonely fighters.

EsSAYs

German and Hungarian workers in post-communism period Eszter Bartha

57 New towns, colonization and Empire. The case of Fertilia Marcel Farinelli

CONTEMPORARY HISTORIANS

83 Historical research and active citizenship: interviewing Eric Foner Daria Frezza and Alessandra Lorini

111 Israeli cinema on Lebanon war Marcella Simoni

\section{Sources}

At Moneda with Salvador Allende Gennaro Carotenuto

Reviews

Neither East nor West: Towards a New History of Twentieth

153 Century Europe?

Marco Bresciani

SHORT REVIEWS

163 Comics in history, history in comics edited by Roberto Bianchi 


\title{
Eric Hobsbawm, un maestro e un amico
}

\author{
Aldo Agosti
}

Eric Hobsbawm, a mentor and a friend. There are many reasons why Eric Hobsbawm has become arguably the most respected historian in the world, recognised if not endorsed on the political right as well as on the left, and one of the few historians of any era to enjoy genuine national and world renown. He was unrivalled both in his knowledge of historic detail and in his extraordinary powers of synthesis, and endowed with an uncommon facility of expression, a lively style and an ability to synthesize complex events, that made his works known in wide circles of non-specialists. At the same time, his widening fame as a historian was accompanied by a growing reputation as a sharp commentator of his times. Till the end he has been remained loyal to a critical but uncompromising interpretation of Marxism, which he used to read the developments of the current economic crisis. One of the first to have discovered Gramsci outside Italy, from the 1970s onward Hobsbawm, who never refused his imprint as an antifascist turned into a Communist, claimed to have become a "spiritual member" of the Italian Communist Party. His influence on the renewal of historical studies in Italy has been remarkable, and left an important mark also in «Passato e presente».

Key words: Eric Hobsbawm, marxism, Gramsci, historiography

Parole chiave: Eric Hobsbawm, marxismo, Gramsci, storiografia

L'ultimo scritto di Eric Hobsbawm, pubblicato dal «Guardian» il 21 settembre, appena dieci giorni prima della sua morte, è l'obituary di una sociologa britannica, studiosa del lavoro e delle relazioni industriali, Dorothy Wedderburn, scomparsa a 87 anni. Nelle righe piene di simpatia e di ammirazione che Hobsbawm le dedica ci sono due passi che meritano di essere citati e che suonano forse meglio in inglese: il primo ricorda la Wedderburn come «a socialist $[\ldots]$, enemy of self-advertisement and an untypical member of the community of "the great and and the good"». Il secondo ne parla come di una donna che «remained steadily, if sometimes sceptically, loyal to the labour movement and the left». È chiaro che risuonano in queste parole echi autobiografici. Se a nessuno verrebbe in mente di negare che per tutta la

«Passato e presente», a. XXXI (2013), n. 88 
sua lunga vita Hobsbawm rimase «tenacemente, anche se talvolta in modo scettico», fedele al movimento operaio e alla sinistra, potrebbe non sembrare corretto definirlo nemico del self-advertisement, perché anche prima della seconda metà degli anni '90, quando raggiunse un'enorme notorietà internazionale, fu sempre interessato al dialogo con storici della sua e di più giovani generazioni, non lesinò le interviste a riviste di storia o a riviste militanti di cultura e di politica; non si sottrasse mai alla responsabilità e anche al gusto di dire la sua sulle questioni riguardanti il mestiere dello storico, ma anche sull'attualità. $\mathrm{Ma}$ in realtà Eric restava una persona schiva, anche se estremamente disponibile verso gli altri, e particolarmente verso le persone più giovani di lui, con un'attenzione mai condiscendente o paternalistica verso i loro interessi e i loro progetti. Non era comunque certo di self-advertisement che aveva bisogno. E meno ancora negli ultimi vent'anni della sua vita, quando divenne probabilmente lo storico più autorevole e più famoso del mondo, anzi - fra gli storici - uno dei pochissimi, o forse il solo, a godere di una fama nazionale e internazionale indiscussa. Il giorno in cui le agenzie battevano la notizia della sua morte, il $1^{\circ}$ ottobre 2012 , una ricerca del suo nome su Google dava la cifra astronomica di 7.780 .000 risultati, quasi dieci volte tanti quelli (820.000) di uno storico, Fernand Braudel, che certo ha contribuito non meno di lui a fornire un senso alla lettura del mondo in cui viviamo. Due settimane dopo, il 15 ottobre, il numero rimaneva ancora altissimo (3.710.000).

Le ragioni di questa straordinaria notorietà sono di natura diversa, e su ciascuna varrebbe la pena di soffermarsi più a lungo. La prima è che la storia scritta da Hobsbawm è affascinante oggi più che mai per due motivi: perché è globale, rivolta cioè a cercare su scala planetaria, e senza limitazioni eurocentriche, i segni delle trasformazioni che scandiscono il tempo; e perché è capace di leggere i nessi tra i diversi campi dell'agire umano, di spiegare una particolare moda artistica o anche un fenomeno di cultura popolare di massa in una rete di relazioni, rivelatrici dello spirito del tempo. La sua passione per il jazz, o il suo interesse giovanile per il fenomeno del millenarismo e del ribellismo, la sua curiosità per i common people, e per gli uncommon people tra loro, non sono curiosità eccentriche, ma frutto di questa concezione della storia, da lui ben descritta in una lezione tenuta al Birkbeck College di Londra nel 1979:

Dal 1950 abbiamo attraversato forse le trasformazioni sociali e culturali più massicce che siano mai esistite a memoria d'uomo, e pochi dubiteranno che esse derivino dagli sviluppi economici e tecnico-scientifici. Pochi dubiteranno che esse siano in qualche misure interconnesse [...]. Ma qual è esattamente il rapporto tra questa trasformazione fondamentale e il rapido declino della popolazione rurale, escluse alcune parti dell'Africa e dell'Asia, la crisi della chiesa cattolica romana, la nascita del rock and roll, il tracollo del movimento comunista mondiale, il disfacimento del matrimonio e dei modelli familiari tradizionali in Occidente, la bancarotta dell'avanguardia artistica, l'interesse degli scienziati per lo sviluppo storico dell'universo, il declino dell'etica puritana del lavoro e del regime parlamentare, e l'insolito spazio 
dedicato alle arti persino in giornali come il Financial Times? E quali sono le interconnessioni fra tutti questi aspetti? Tali domande sono di enorme interesse e importanza e di difficoltà altrettanto enorme. Tuttavia gli storici devono provare di nuovo ad affrontarle. Andranno più avanti di Montesquieu e dovrebbero andare più avanti di Marx (De historia, Rizzoli, Milano 1997, p. 86).

Una seconda ragione è che Hobsbawm, come ha ricordato il suo allievo e amico Donald Sassoon, eccelleva in quel genere che i francesi chiamano haute vulgarisation: un termine che soprattutto in passato faceva storcere il naso a molta parte dell'accademia, ma del quale lui era personalmente orgoglioso. Gli sarebbe piaciuto l'epitaffio che gli ha dedicato il segretario del Labour Party, Ed Miliband: «ha messo centinaia di anni di storia alla portata di centinaia di migliaia di persone, ha portato la storia fuori dalla torre d'avorio e dentro la vita della gente» («The Guardian», $1^{\circ}$ ottobre 2012).

Una terza ragione è che, come ha scritto Giovanni De Luna, «Hobsbawm con il suo lavoro ha disegnato una sorta di mappa che ci consente di percorrere gli intricati itinerari della storia dell'umanità negli ultimi due secoli», coniando categorie e concetti che sono diventati altrettanti punti obbligati del dibattito storiografico («La Stampa», 2 ottobre 2012). Uno di questi, in particolare, è quello che gli ha fatto raggiungere l'apice della sua fama: il «secolo breve», sottotitolo del libro The Age of Extremes, che è diventato in molte delle 37 lingue in cui è stato tradotto più conosciuto del titolo. La periodizzazione a cui questo corrisponde (1914-1991) è sicuramente per alcuni aspetti discutibile, ma ha una forza di attrazione non dissimile da quella, da lui stesso magistralmente spiegata in un libro quasi altrettanto celebre, che acquisiscono le tradizioni "inventate". Anche chi non la condivide è stato costretto a farci i conti, perché davvero il corso degli eventi successivi al suo termine ad quem ha segnato l'inizio di un'altra storia, davvero quello si è rivelato un punto di svolta decisivo nella dislocazione degli equilibri internazionali, economici non meno che politici. Così l'autobiografia che Hobsbawm ha pubblicato pochi anni dopo, nel 2002 - una delle più avvincenti e affascinanti autobiografie di storico che siano mai state scritte - ha finito per sovrapporre gli «anni interessanti» da lui vissuti proprio con il «secolo breve» che ha "inventato", con un effetto di ricaduta impressionante sulla popolarità di entrambe le opere.

Ma Hobsbawm non sarebbe stato Hobsbawm se, superati ampiamente gli ottant'anni, non avesse avuto la capacità di sopravvivere al suo «secolo breve» e di applicare la profondità del suo ingegno di storico all'interrogazione del presente e del futuro, adempiendo con rigore a quella funzione che, per lui, era stato il merito maggiore di Gramsci: «averci insegnato che lo sforzo per trasformare il mondo non solo è compatibile con una riflessione storiografica originale, sottile e lucida ma è impossibile senza di essa» (Come cambiare il mondo. Perché riscoprire l'eredità del marxismo, Rizzoli, Milano 2011, p. 343). Lo ha fatto, come ha osservato Gianpasquale Santomassimo, passando gli ultimi anni della sua vita «a discutere, limare, correggere la sua 
interpretazione, alla luce dei nuovi avvenimenti che cambiavano il quadro del mondo descritto nell'ultimo capitolo, La frana, che seguiva improvvisamente l'età dell'oro dell'Occidente» («il manifesto», 2 ottobre 2012, p. 11).

Questo è stato vero fino all'ultimo. Lo si è potuto constatare il 4 luglio 2012, quando Eric e sua moglie Marlene hanno dato una festa a Londra, per il $95^{\circ}$ compleanno di lui, $1^{\prime} 80^{\circ}$ di lei e le loro nozze d'oro. L'indomito anticonformismo di entrambi faceva capolino già nella mail in cui l'annunciavano agli invitati, tenendo ironicamente a sottolineare che la coincidenza con la data della festa nazionale degli Stati Uniti non era voluta. La festa è riuscita benissimo, anche se fra i partecipanti aleggiava la sottile malinconia associata all'idea del commiato. Hobsbawm appariva fragile e provato, ma la sua lucidità mentale era assolutamente intatta. Fece, con voce chiara e non flebile, un discorso che ripercorreva le tappe della sua vita e del suo lavoro, soffermandosi soprattutto sul tema degli affetti familiari e dell'amicizia. E poi con un'impennata della sua proverbiale ironia, aggiunse che accettava la sua vecchiaia perché non poteva fare altrimenti, ma anche - con un sorriso appena accennato che illuminò il suo sguardo ancora limpido - perché gli riservava qualche soddisfazione, come quella di vedere il «Financial Times» riconoscere che occorreva guardare a Marx per capire come ha funzionato e funziona il mondo. Così, ancora una volta, confermava di essere anche lui, e più di chiunque altro, veramente «an untypical member of the community of the great and the good»: un marxista non pentito.

L'ultima parte della vita di Hobsbawm è infatti caratterizzata da una riflessione costante sulla crisi che a diverse riprese, e con particolare intensità dopo il 2007, ha scosso l'economia capitalistica: una crisi che lo ha indotto a guardare a Marx, come ha notato Sassoon, non come al teorico della rivoluzione mondiale e del ruolo egemone del proletariato, ma al pensatore capace di anticipare le grandi linee dell'ultima (ci guarderemo bene dal definirla «suprema») forma che il capitalismo moderno ha assunto, quella che è invalso l'uso di definire "globalizzazione" e che con ogni evidenza attraversa una fase di forte tensione. A 94 anni ha pubblicato quello che è per ora il suo ultimo libro (sappiamo però che ne aveva in preparazione un altro, che uscirà nel 2013, una raccolta di saggi su temi prevalentemente culturali): Come cambiare il mondo. Perché riscoprire l'eredità del marxismo, una raccolta di scritti, alcuni dei quali inediti, in cui rivendica ancora una volta l'attualità del pensatore di Treviri: «Non possiamo prevedere le soluzioni ai problemi che il mondo deve affrontare nel XXI secolo, ma se si vuole avere una chance di successo bisogna porsi le stesse domande che si pose Marx, anche se non si vogliono accettare le risposte dei suoi vari discepoli» (Come cambiare il mondo, p. 13).

È stato osservato che Hobsbawm «ha raggiunto l'apice della sua reputazione nel momento in cui le idee socialiste e i progetti che avevano animato gran parte dei suoi scritti per più di mezzo secolo erano storicamente allo sbando» («Mainstream», 6 ottobre 2012). L'osservazione è solo in parte fondata, perché la produzione di Hobsbawm si era nutrita di ben altra sostanza 
che di elegiache rappresentazioni del socialismo, realizzato o da realizzarsi. È vero però che egli era consapevole che era (temporaneamente?) scomparsa la fiducia condivisa dai protagonisti delle grandi rivoluzioni dell'età contemporanea che fosse possibile cambiare l'ordine sociale esistente e sostituirlo con uno migliore. Scrisse di questo spesso e con grande lucidità, facendo ampie concessioni più al pessimismo della ragione che all'ottimismo della volontà: nella sua Marx Memorial Lecture del 1978, intitolata significativamente The Forward March of Labour Halted?, sorprese i suoi ascoltatori (e poi i lettori dell'ampia raccolta di saggi pubblicata sotto quel titolo) lasciando trasparire la crescente convinzione che i movimenti operai europei avessero cessato di svolgere il ruolo di agenti della trasformazione che il marxismo aveva loro assegnato. Ultimamente, sembrava essere diventato ancora più pessimista: «La sinistra non ha più niente da dire, non ha un programma da proporre disse in un'intervista rilasciata all' «Espresso» e uscita il 16 maggio 2012 Quel che ne rimane rappresenta gli interessi della classe media istruita, e non sono certo centrali nella società».

Tuttavia, negli ultimi anni, è apparso chiaro che la gravissima crisi e il declino dei movimenti operai organizzati non inficiano la validità e la preveggenza di molti aspetti dell'analisi di Marx sui meccanismi dell'economia capitalistica. Hobsbawm, che non aveva mai rinunciato a sottolinearle, ha potuto prendersi una rivincita non di poco conto sul pensiero unico dominante e anche vedere convalidata a posteriori la propria tesi che troppi critici del $\mathrm{Se}$ colo breve avevano liquidato superficialmente come wishful thinking: quella che il capitalismo si fosse riformato e "incivilito" grazie alla concorrenza del socialismo, e che in quella concorrenza avesse trovato la spinta per garantire a buona parte del mondo - per oltre un quarto di secolo - un forte sviluppo economico e una diminuzione delle diseguaglianze sociali. Egli ha così potuto scrivere in un'intervista del 10 aprile 2009 al «Guardian»: «l'impotenza [...] imprigiona sia coloro che credono in un capitalismo di mercato, puro e senza Stato, sia coloro che credono in un socialismo pianificato, incontaminato dalla ricerca privata del profitto. Gli uni e gli altri hanno fallito. Il futuro, come il presente e il passato, appartiene alle economie miste in cui il pubblico e il privato, in un modo o nell'altro, si intreccino. Ma come? Questo è il problema di tutti oggi, ma lo è particolarmente per la sinistra».

Marxista non pentito, dunque, anche se sempre aperto a cercare nel pensiero di Marx domande e risposte che andassero oltre Marx stesso. Ma anche comunista non pentito: un tratto della sua identità che ha fatto scorrere fiumi d'inchiostro per anni e riempito molte pagine di giornale anche in occasione della sua morte. Non torneremo qui su questo aspetto, avendone parlato ampiamente in un profilo che «Passato e presente» gli ha dedicato nel 2011 (n. 82). Due punti soltanto meritano, per ragioni diverse, di essere toccati. Il primo è che, anche se non ha mai rinnegato la sua appartenenza comunista, anzi l'ha rivendicata, su di essa Hobsbawm ha avuto espressioni autocritiche anche severe, spesso ignorate da chi lo ha sempre rimproverato della sua presunta "complicità". Il secondo punto è che il comunismo di Hobsbawm era qualco- 
sa di particolare. Lo era sempre stato, per la verità: se non altro nel senso che aveva molto marginalmente intaccato il rigore critico della sua analisi storica, tanto che volutamente a lungo si era tenuto lontano dalla storia dei movimenti comunisti del ' 900 e delle società socialiste; ma ancor più lo divenne dopo il 1956, quando, come egli stesso ha scritto, si considerò soprattutto un «membro spirituale» del Pci. E sul legame di Hobsbawm con l'Italia, con la cultura storica italiana è giusto spendere ancora qualche parola.

A Eric l'Italia piaceva, come in generale gli piacevano i paesi latini: in Italia veniva spesso, e volentieri. Mi confessò che fra le centinaia d'inviti per dibattiti e conferenze che gli giungevano ogni anno, aveva un occhio di riguardo per quelli provenienti dal Bel Paese. Gli piaceva il nostro cibo, moltissimo la nostra opera lirica: in Italia sua moglie Marlene aveva vissuto diversi anni e conservava molti amici. Gli piaceva, negli anni '60 e ' 70 , il clima di vivacità culturale che si respirava nella sinistra italiana, lo sforzo del Partito comunista di pensare in termini originali quella che allora si chiamava «la fuoriuscita dal capitalismo». Nel 1975, alla vigilia delle elezioni del 15 giugno 1976 che segnarono l'apice della forza parlamentare del Pci, intervistò Giorgio Napolitano (di cui è sempre rimasto amico), e ne uscì un dialogo a tutto campo di grande respiro e interesse ancora attuale (G. Napolitano, Intervista sul Pci, a cura di E.J. Hobsbawm, Laterza, Roma-Bari 1975). Non c'è dubbio che in questo percorso di parziale "italianizzazione" di Hobsbawm - un termine che lo avrebbe fatto sorridere ma che non credo avrebbe respinto, salvo rifiutare la cittadinanza dell'Italia di Berlusconi - un ruolo importantissimo abbia avuto la scoperta di Gramsci, come emerge chiaramente dalla sua autobiografia e dagli stessi suoi primi scritti, e come è stato esplicitamente riconosciuto in un suo intervento del 2007:

Rimasi colpito quasi immediatamente non tanto dall'approccio politico di Gramsci, che peraltro all'epoca era molto originale per un marxista, ma soprattutto dal suo approccio alla storia delle classi subalterne, alla storia delle classi popolari. Sotto certi riguardi i miei primi scritti storici erano paralleli a quelli di Gramsci in questa direzione. [...] Il mio rapporto personale con Gramsci è stato, in un certo senso, fondativo: Gramsci è una delle maggiori fonti di ispirazione del mio lavoro di storico. Allo stesso tempo egli rappresenta anche una essenziale fonte di ispirazione delle mie idee sulla politica, perché Gramsci è stato uno dei pochissimi, forse l'unico tra i marxisti e i comunisti, a scoprire che l'oggetto della politica non è soltanto la questione di come prendere il potere e mantenerlo, ma che c'è invece molto altro oltre a questo (intervista raccolta da Giorgio Baratta, in www.millepiani.net/archivesfilosofici/ 2007/05/05/gramsci_grazie_ai_quaderni_sono_uno_storico_eric_j_hobsbawm.html).

A Gramsci, anzi a "Nino" (così gli si rivolge, come facevano i famigliari e gli amici più stretti), ha indirizzato nel 2007 una commovente lettera, registrata in video da Giorgio Baratta, in cui, curiosamente ma non troppo, insiste molto sulla "sardità" di Gramsci, che dichiara di avere capito attraverso l'intensa frequentazione di un'altra regione "periferica", il Galles. Desta qualche sorpresa, ma non c'è ragione di non crederci, che Hosbawm abbia 
sentito parlare per la prima volta di Gramsci, come racconta, da alcuni «dei molti soldati britannici che tornarono in patria dopo aver combattuto la guerra in Italia, ove avevano sentito parlare di lui: [...] da uomini come il poeta Hamish Henderson, ottimo scrittore, gran bevitore, scozzese, che fu tra i primi a tradurre le Lettere dal carcere, e da diverse altre persone, che mi sollecitarono a prendere personalmente contatto con i suoi testi». In ogni caso, e percorrendo il cammino in senso inverso, è probabile che gli scritti di Hobsbawm siano stati uno dei tramiti della diffusione di Gramsci nel mondo, specialmente in quello anglofono. Ricorrendo di nuovo a quella spia molto rudimentale che è il numero di segnalazioni su Google (una spia peraltro che Eric non disdegnava di usare spesso), non è probabilmente un caso, ma l'effetto di un reciproco trascinamento, che alla cifra sopra richiamata di 7.780.000 di citazioni per Hobsbawm la sera della sua morte corrisponda, nello stesso momento, un numero molto maggiore del consueto di citazioni di Gramsci (5.650.000, rispetto a 3.840 .000 di quindici giorni dopo).

Il terreno su cui Hobsbawm ha incontrato l'Italia, dunque, è stato quello arato dal pensiero di Gramsci (del Gramsci dei Quaderni del carcere, perché non pare fosse molto interessato al Gramsci ordinovista e leninista) e reso attraente (dal punto di vista di un marxista critico) dalla presenza di un partito comunista anomalo, una sorta di ibridazione fra comunismo e socialdemocrazia che sembrava capace di valorizzare gli aspetti migliori di ciascuno di loro e di ciascuno in grado di esorcizzarne (o di nascondere) le debolezze. A sua volta, il terreno su cui la cultura storica italiana ha scoperto Hobsbawm è stato più variegato e composito e forse, comprensibilmente, diverso per ciascuno.

Mi è già capitato di richiamare l'influenza che ebbe su un'intera generazione di storici italiani il suo saggio tradotto nel 1973 su «Quaderni storici», Dalla storia sociale alla storia della società: nel quale tracciava una sorta di mappa che conserva ancor oggi quasi intatta la sua capacità di orientare la ricerca, individuando come terreni di approfondimento una serie di temi o complessi di questioni al confine tra diverse scienze sociali. Ma quello scritto (e in generale il suo lavoro) non si limitarono ad esercitare una grande forza di seduzione su quella generazione di storici che, secondo la celebre espressione di Nicola Gallerano, «si erano addormentati storici politici e si erano svegliati storici sociali»: non tutti gli storici politici degli anni ' 70 , particolarmente quelli del movimento operaio, entrarono in letargo. E alcuni irrobustirono il loro lavoro con tonificanti iniezioni di storia sociale: tenendo in particolare considerazione l'insegnamento di Hobsbawm, dal quale poterono attingere preziosi spunti sia per una storia politica rinnovata, sia per una storia sociale non svuotata dei suoi aspetti politici.

Un riflesso indiretto ma significativo dell'incontro fra Hobsbawm e la cultura storica italiana furono le ambiziose imprese editoriali di Einaudi di cui fu, insieme ad altri studiosi di rilievo, animatore intellettuale: prima la Storia del marxismo in quattro volumi, pubblicata fra il 1978 e il 1982: nella quale scrisse personalmente numerosi saggi, della cui solidità e longevità era evidentemente e a giusta ragione convinto, tanto da ripubblicarli tutti trent'anni 
dopo in Come cambiare il mondo; poi, meno noto forse della precedente ma in realtà molto importante per la vera e propria sfida intellettuale che si dimostrava in grado di raccogliere, il gigantesco quinto volume della Storia d'Europa (L'età contemporanea, secoli XIX-XX), curato insieme a Paul Bairoch e uscito nel 1996.

È abbastanza evidente che di questo incontro resta una traccia anche in «Passato e presente». Nell'impostazione della rivista, fin dalla fondazione ormai trent'anni fa, erano presenti molti stimoli riconducibili all'impronta di Hobsbawm, anche se non solo alla sua: quelli che un osservatore intelligente del panorama delle riviste italiane fra gli anni '70 e gli anni '90, Leonardo Rapone, ha egregiamente riassunto come segue:

allargamento della periodizzazione [...] risalendo all'indietro dal Novecento alla duplice rivoluzione della fine del XVIII secolo; apertura al dibattito storiografico internazionale e alle novità metodologiche che ne erano emerse [...]; valorizzazione della storia sociale, nel rifiuto però di una gerarchia delle forme della conoscenza storica; la capacità di connettere criticamente l'oggetto della ricerca ai processi storici costitutivi dell'età contemporanea, rifuggendo dall'assolutizzazione del fatto e da approcci fortemente ideologici (L'età contemporanea, «Studi storici», 53, 2012, n. 2, pp. 326-27).

Non fosse che per questo, «Passato e presente» ha un debito inestinguibile di gratitudine verso Eric Hobsbawm: al quale si aggiunge quello di averlo avuto fra i membri del suo comitato scientifico, qualche volta perfino fra $\mathrm{i}$ suoi collaboratori (anche con un'intervista che ci rilasciò in occasione del suo $80^{\circ}$ compleanno). Per cui si può forse concludere questo ricordo, non facile da scrivere per chi gli è stato anche personalmente molto legato, volgendo al maschile le parole da lui usate per salutare Dorothy Wedderburn: «For his survivors, his greatest gift was friendship». 


\title{
Volksgemeinschaft tra storiografia e memoria
}

\author{
a cura di Paolo Fonzi
}

\begin{abstract}
Volksgemeinschaft between historiography and memory. In recent years a debate has been taking place among historians of Nazism about the role played by the concept of Volksgemeinschaft (Popular community) in the Third Reich. Though historians agree on the general relevance of this ideal for the National Socialist regime, the debate revolves around the question, whether this concept can be also useful employed for understanding material, political and social processes. Three German historians - Michael Wildt, Hans Mommsen and Ulrich Herbert - present their argumentations in favour or against the use of the concept of Volksgemeinschaft in this sense. Their stances imply different evaluations of how extensive and intense was the participation of the ordinary Germans in Nazi policy.
\end{abstract}

Key words: National socialism, consent, social history of Germany, political violence Parole chiave: nazionalsocialismo, Terzo Reich, consenso, storia sociale della Germania, violenza politica

\section{Paolo Fonzi}

Se il dibattito tra strutturalisti e intenzionalisti che ha impegnato gli storici del nazionalsocialismo a partire dagli anni '70 è considerato da molti un relitto del passato, da alcuni anni all'interno degli studi sul Terzo Reich si delinea una nuova spaccatura. Questo nuovo dibattito verte sulla valutazione di una parola che racchiude tutti i contenuti programmatici che il Terzo Reich iscrisse sulle sue bandiere: Volksgemeinschaft. In italiano questo termine viene spesso tradotto «comunità di popolo» $\mathrm{o}$ 《comunità popolare», ma ogni traduzione comporta inevitabilmente una perdita di senso, perché in tedesco la parola fa risuonare simultaneamente una storia, una polisemia, una serie di termini oppositivi. Per buona parte questa difficoltà sta nel primo termine del composto, Volk (popolo), che in tedesco ha sempre rimandato a un'unità profonda ed essenziale, con una pregnanza che i corrispettivi termini in altre lingue non hanno. Meno complessa la traduzione del secondo termine, Gemeinschaft (comunità), che una tradizione sociolo-

«Passato e presente», a. XXXI (2013), n. 88 
gica recepita a livello internazionale ha reso più comprensibile al di fuori dell'area di lingua tedesca.

Il concetto di Volksgemeinschaft designava in sintesi il progetto sociale del nazionalsocialismo, la trasformazione della società in comunità, la ritrovata unità della nazione sottratta alla decadenza moderna. Il Vokabular des Nationalsozialismus del 1998, che illustra le più significative espressioni del linguaggio politico del Terzo Reich, la definisce una «comunità di vita (Lebensgemeinschaft) che scaturisce da una comunità di sangue, di destino, e da una comunità di fede nazionalsocialista, in cui classi, partiti, contrapposizioni di ceto e interessi individuali devono esser superati in nome dell'utile collettivo di tutti i compagni del popolo».

Non sempre connotato in senso razzista, il termine - la cui prima attestazione nota è in una traduzione tedesca del 1791 di uno scritto di John Locke - era scarsamente diffuso nell'800. Solo dopo il 1914 e in modo significativo dopo il 1933 crebbe il ricorso a questo concetto, soprattutto da parte della destra politica. Il Vokabular individua tre aree principali in cui si può suddividere il campo semantico del termine Volksgemeinschaft nell'accezione nazionalsocialista: esso indica una «comunità di sangue definita razzialmente», una «comunità sociale solidale tra lavoratori intellettuali e manuali, che non conosce contrapposizione d'interessi e che si materializza in particolare nella comunità d'impresa costituta dai capi d'impresa e dal seguito (Gefolg$s c h a f t) »$, infine un nuovo termine giuridico, ovvero la comunità che in ultima analisi costituisce la fonte del diritto secondo il principio: «diritto è ciò che giova al popolo» 1 .

Nessuno storico nega che l'idea di Volksgemeinschaft sia un elemento portante della storia del Terzo Reich. Ciò che da alcuni anni è oggetto di discussione su riviste scientifiche e in monografie è il valore che questo concetto deve avere nell'interpretazione generale del fenomeno: si trattò di pura propaganda o di realtà? $\mathrm{E}$ in che senso va intesa questa realtà? In quale misura l'adesione alla promessa di realizzare la Volksgemeinschaft influenzò il comportamento della popolazione tedesca e quanto estesa fu questa adesione?

Un nutrito gruppo di storici sostiene la necessità di porre tale concetto al centro dell'interpretazione della società tedesca durante il nazionalsocialismo. Per illustrare la loro posizione si può partire da un piccolo volume edito nel 2009 da Michael Wildt e Frank Bajhor, una sorta di manifesto di questa corrente $^{2}$. Nell'introduzione i curatori chiariscono che la Volksgemeinschaft non va intesa come realtà sociale tout court quanto piuttosto come «promessa» (Verheissung). Non per questo, però, si deve negare che tale promessa avesse effetti sociali "reali" e decisivi nella storia del regime. Il concetto di Volksge-

${ }^{1}$ C. Schmitz-Berning, Vokabular des Nationalsozialismus, De Gruyter, Berlin 1998, p. 654.

2 F. Bajhor-M. Wildt (hrsg.), Volksgemeinschaft. Neue Forschungen zur Gesellschaft im Nationalsozialismus, Fischer Taschenbuch Verlag, Frankfurt am Main 2009. Il volume contiene gli interventi presentati alla sezione dello Historikertag del 2008, organizzata dagli stessi Bajohr e Wildt. 
meinschaft aveva il pregio di prestarsi a multiformi interpretazioni e quindi di racchiudere un progetto sociale complessivo incardinato su due dimensioni parallele e inscindibili: da un lato una tendenza all'uguaglianza in senso verticale, cioè la promessa del superamento di ogni barriera nella società razzialmente purificata, dall'altro un processo di violenta differenziazione orizzontale fatto di esclusione e violenza verso i Gemeinschaftsfremde, i soggetti estranei alla comunità. A questo progetto i tedeschi aderirono in forme diverse ma comunque in modo massiccio, partecipando alla sua realizzazione non solo come esecutori ma anche come volenterosi carnefici. Questa interpretazione ha suscitato notevoli reazioni critiche e negli ultimi 3-4 anni si è aperta un vera e propria discussione tra i maggiori storici del nazionalsocialismo ${ }^{3}$.

La principale critica mossa agli esponenti della corrente della Volksgemeinschaft è di prestare credito alla vuota propaganda egualitarista del regime. Inoltre, si rimprovera loro di leggere la società tedesca in chiave monista, sottovalutando l'autonomia di istituzioni e nicchie sociali rispetto alla penetrazione del regime, insomma di riassumere il Terzo Reich in una sola parola, Volksgemeinschaft, che, in definitiva, sarebbe sinonimo di consenso ${ }^{4}$. Gli esponenti della corrente rispondono a questa critica sottolineando che, come strumento di analisi della società tedesca, la Volksgemeinschaft va intesa in senso prasseologico, è cioè un concetto programmatico che vive solo nella sua realizzazione e appropriazione da parte degli attori sociali 5 . In questa appropriazione si manifesterebbero l'irriducibile complessità del sociale e le multiformi esperienze dei tedeschi durante il nazionalsocialismo. I tre interventi che seguono intendono offrire al lettore italiano un quadro del ventaglio di posizioni espresse nel dibattito in corso: Michael Wildt è il più noto esponente della corrente della Volksgemeinschaft, Ulrich Herbert ne è un critico moderato, Hans Mommsen in diverse occasioni ha attaccato duramente questo approccio. Prima di "dar loro la parola" svilupperò alcune considerazioni introduttive.

La questione della Volksgemeinschaft si pone su due piani fortemente intrecciati: da un lato il piano prettamente scientifico, dall'altro la Vergangenheitsaufarbeitung (elaborazione del passato) e il problema dell'identità nazionale tedesca. Come detto, alla valutazione del concetto di Volksgemeinschaft è legata la questione del consenso dei tedeschi verso il nazionalsocialismo. Nei primi decenni dopo la fine della seconda guerra mondiale la storio-

${ }^{3}$ Cfr. D. Schmiechen-Ackermann (hrsg.), «Volksgemeinschaft»: Mythos, wirkungsmächtige soziale Verheißung oder soziale Realität im «Dritten Reich»? Zwischenbilanz einer kontroversen Debatte, Schöningh, Paderborn 2012. Nel marzo 2010 si è tenuta presso il Deutsches Historisches Institut di Londra una conferenza internazionale sul tema, i cui atti sono in corso di pubblicazione.

${ }^{4}$ I. Kershaw, «Volksgemeinschaft». Potenzial und Grenzen eines neuen Forschungskonzepts, «Vierteljahrshefte für Zeitgeschichte», 2011, n. 1, pp. 1-17.

5 M. Wildt, «Volksgemeinschaft». Eine Antwort auf Ian Kershaw, «Zeithistorische Forschungen/Studies in Contemporary History», 2001, n. 1 (www.zeithistorische-forschungen.de/ 16126041-Wildt-1-2011). 
grafia tedesca non ha attribuito al concetto di Volksgemeinschaft alcuna sostanza di realtà. La rimozione delle responsabilità dei tedeschi comuni e l'auto-vittimizzazione collettiva erano il carattere distintivo della memoria del nazionalsocialismo nella Bundesrepublik degli anni '50. Per questo le letture del nazionalsocialismo di quel periodo mettevano in primo piano gli aspetti repressivi del regime e la sua presa totalitaria sulla società. In quegli anni parlare di Volksgemeinschaft attribuendole più di un valore meramente propagandistico era, sottolinea Norbet Frei, un tabù ${ }^{6}$. La storiografia della Ddr, pur utilizzando criteri interpretativi nettamente diversi, tendeva ugualmente a mettere in ombra l'adesione dei tedeschi al regime. Negli anni '60 e '70 la società tedesco-occidentale cominciò a confrontarsi in modo più aperto con il proprio passato, eppure anche nella storiografia rimaneva un forte sospetto verso l'ideologia e i miti "positivi” del Terzo Reich.

In quegli stessi anni si svolse un importante dibattito sul rapporto tra nazionalsocialismo e classe operaia. Nella sua opera maggiore del 1975, Arbeiterklasse und Volksgemeinschaft ${ }^{7}$, Timothy Mason sosteneva che la classe operaia in fondo era rimasta estranea alle promesse del regime, praticando forme di opposizione sotterranee e prive di diretta espressione politica. Dietro a tutto questo vi era la discussione degli anni '60 sul rapporto tra modernizzazione e nazionalsocialismo. Ralf Dahrendorf e David Schoenbaum lanciarono allora una tesi molto discussa secondo cui il nazionalsocialismo aveva avuto effetti modernizzatori, tra i quali l'accresciuta mobilità e un maggiore egualitarismo sociali ${ }^{8}$. Questa proposta interpretativa provocò una levata di scudi da parte di molti storici. «A ben guardare - scriveva Heinrich August Winkler recensendo il libro di Mason - le prove più decisive della Volksgemeinschaft nazionalsocialista sono per lo più quelle che dovrebbero rendere scettici proprio sociologi come Dahrendorf: cioè le testimonianze degli stessi nazionalsocialisti» 9 .

Solo negli anni '80 la storiografia iniziò a dissodare il terreno della "storia dell'esperienza" dei tedeschi nel Terzo Reich e gli effetti di consenso che tale esperienza aveva sviluppato. Molti studi di quegli anni ${ }^{10}$, ad esempio le ricerche di storia orale dirette da Lutz Niethammer ${ }^{11}$, corressero decisamente

${ }^{6}$ N. Frei, Volksgemeinschaft. Erfahrungsgeschichte und Lebenswirklichkeit der Hitler-Zeit, in Id., 1945 und wir. Das dritte Reich im Bewusstsein der Deutschen, Beck, München 2005, pp. $107-28$

7 T.W. Mason, Arbeiterklasse und Volksgemeinschaft: Dokumente und Materialien zur deutschen Arbeiterpolitik 1936-1939, Westdeutscher Verlag, Opladen 1975.

8 R. Dahrendorf, Gesellschaft und Demokratie in Deutschland, Piper, München 1965; D. Schoenbaum, Hitler's Social Revoution. Class and Status in Germany 1933-1939, Doubleday, New York 1966.

9 H.A. Winkler, Vom Mythos der Volksgemeinschaft, «Archiv für Sozialgeschichte», 17 (1977), pp. 484-85.

10 Cfr. U. Herbert, Arbeiterschaft im "Dritten" Reich. Zwischenbilanz und offene Fragen, «Geschichte und Gesellschaft», 3 (1989), pp. 320-60.

${ }^{11}$ L. Niethammer (hrsg.), Lebensgeschichte und Sozialkultur im Ruhrgebiet 1930 bis 1960, v. 1, "Die Jahre weiß man nicht, wo man die heute hinsetzen soll", Dietz, Berlin 1983. 
l'immagine tradizionale secondo cui la classe operaia si era mantenuta distante dal regime. Accanto a fattori che inducevano gli operai a mantenere un'effettiva distanza (controllo sociale e poliziesco, politica dei salari) vi erano forti elementi produttori di consenso (eliminazione della disoccupazione, consolidamento della posizione sociale di alcuni settori della popolazione lavoratrice, successi in politica estera). Sotto il nazismo si dissolse progressivamente il tradizionale contesto sociale operaio che negli anni '20 aveva tenuto insieme "quotidiano" e coscienza politica. Per questo molti settori della classe operaia divennero vulnerabili alle promesse di mobilità e avanzamento sociale del regime. Sebbene le barriere sociali non fossero state cancellate, esse determinarono sempre meno l'esperienza e la percezione di sé dei tedeschi. Sulla base di queste indagini, dunque, gli storici misero sempre più in luce come il progetto nazionalsocialista fosse stato un significativo collante sociale e avesse sviluppato una forte identificazione con il regime.

Negli stessi anni nella storiografia tedesca si verificò una forte svolta epistemologica attraverso la diffusione della storia del quotidiano e la parallela, importante attività delle Geschichtswerkstätte, associazioni dedite all'elaborazione della storia "dal basso" diffusesi nella Brd degli anni '80. Il maggiore esponente della storiografia tedesca del quotidiano, Alf Lüdtke, ha analizzato in diversi saggi ${ }^{12}$ la società negli anni del Terzo Reich usando come strumento di lettura la categoria di «appropriazione» (Aneignung), l'elemento metodologico portante del concetto di quotidiano da lui proposto. Questo approccio mira a superare una concezione meramente verticale del potere, osservando come gli individui non eseguano e realizzino in modo automatico le prescrizioni implicite nella struttura sociale, ma nel realizzarle si approprino di tali strutture modificandole e, per così dire, "attualizzandole". Il concetto di appropriazione rimanda quindi a una visione dei rapporti sociali che si è affermata potentemente nella cultura europea e americana degli ultimi 20-30 anni e che ha trovato una sistematizzazione nel pensiero di Michel De Certeau e nel filone dei cultural studies.

Un mutamento complessivo nella percezione del nazionalsocialismo, dunque, è iniziato negli anni ' 80 e si è rafforzato secondo molti osservatori (soprattutto secondo gli stessi esponenti della corrente della Volksgemeinschaft) dopo la riunificazione tedesca. Il dibattito in corso è una chiara espressione di questa svolta, legata sia a una transizione generazionale che a un mutamento della "cultura della memoria". Non essendovi spazio per approfondire questo argomento mi limito a rilevare che negli studi sul Terzo Reich è venuta meno una visione generalmente accettata dalla storiografia precedente, secondo la quale il nazismo giunse al potere "dall'esterno" ed erose in un percorso "a stadi" le vecchie strutture socio-politiche fino a culminare negli anni

12 Per brevità rinvio al solo A. Lüdtke, The Appeal of Exterminating “Others": German Workers and the Limits of Resistance, «The Journal of Modern History», s.i. Resistance, 1992, pp. 46-67. 
della guerra nella loro completa "occupazione". Gran parte della storiografia recente, invece, mette in evidenza l'auto-mobilitazione di importanti settori dello stato e della società, la loro spontanea rifunzionalizzazione e convergenza verso gli obiettivi del regime. La radicalizzazione della politica nazionalsocialista che culminò nella Soluzione finale non è più dunque vista principalmente come il portato della lenta presa del partito sulla società o come un prodotto della "radicalizzazione cumulativa", quanto come il frutto del convergere della società verso il crimine.

Dal punto di vista della Vergangenheitsaufarbeitung queste nuove tendenze della ricerca sono parte di una cultura della memoria di cui si sono fatti interpreti i governi della "Repubblica di Berlino", in primo luogo quello rosso-verde ma anche, seppur con un certo spostamento di accenti, quelli successivi. L'idea portante della politica della memoria di questi governi è che al centro dell'identità nazionale della Germania riunificata vi debba essere un'inequivocabile ammissione di colpa della nazione dei persecutori. In questa ottica le vecchie interpretazioni del nazionalsocialismo sono accusate di aver, spesso involontariamente, attribuito la responsabilità al solo Hitler o ad anonime strutture sociali distogliendo lo sguardo dal comportamento dei tedeschi comuni. Su questa linea convergono larghe fette del mondo intellettuale tedesco di diverso colore politico, non senza però accesi conflitti di cui i dibattiti degli ultimi anni - da quello sui crimini della Wehrmacht alla recente disputa sulle tesi di Götz Aly - sono eloquente testimonianza.

\section{Michael Wildt, Una nuova prospettiva sulla società nazionalsocialista}

Il concetto di Volksgemeinschaft non è genuinamente nazionalsocialista. Esso, infatti, deve la sua fortuna alla prima guerra mondiale ${ }^{13}$. La frase pronunciata da Guglielmo II nel 1914, secondo cui da quel momento in poi egli non conosceva più partiti ma solo tedeschi, ebbe grande risonanza, perché seppe esprimere il desiderio di eguaglianza e di inclusione di molti tedeschi. Proprio gli ebrei e i socialdemocratici speravano di essere finalmente accettati come eguali dalla maggioranza della società per il loro comportamento patriottico. Nonostante si manifestassero ben presto crepe nella società di guerra, la Volksgemeinschaft rimase una formula potente che poteva sempre riallacciarsi al mito dell'unità dell'estate del 1914.

Già prima del 1933 la Volksgemeinschaft divenne in Germania, come rileva Hans-Ulrich Thamer, una «formula interpretativa dominante in ambito politico» ${ }^{14}$. I partiti liberali ne sottolineavano l'accezione di armonia sociale, in-

13 Cfr. S. Bruendel, Volksgemeinschaft oder Volksstaat. Die "Ideen von 1914" und die Neuordnung Deutschlands im Ersten Weltkrieg, Akademie Verlag, Berlin 2003; J. Verhey, Der "Geist von 1914" und die Erfindung der Volksgemeinschaft, Hamburger Edition, Hamburg 2000 .

${ }^{14}$ H.-U. Thamer, Volksgemeinschaft: Mensch und Masse, in R. van Dülmen (Hrsg.), Erfindung des Menschen. Schöpfungsträume und Körperbilder 1500-2000, Böhlau, Wien 1998, p. 
clusiva, «al di sopra delle classi». Per i socialdemocratici il concetto di classe lavoratrice si era allargato a quello di popolo dei produttori, che si contrapponeva ad una piccola e ingiustamente potente minoranza di capitalisti monopolistici e di grandi proprietari terrieri. E questa stessa minoranza, se si fosse dedicata a un vero e proprio lavoro, sarebbe potuta diventare parte di una Volksgemeinschaft socialista. Nei discorsi del presidente del Reich Friedrich Ebert la Volksgemeinschaft aveva un posto fisso e voleva dire inclusione di tutti i produttori.

Al contrario la destra politica, specialmente i nazionalsocialisti, interpretò la Volksgemeinschaft, nonostante tutta la retorica di inclusione, soprattutto nella sua dimensione di esclusione. In primo piano non vi era tanto la questione di chi vi appartenesse, quanto il problema di chi non avesse diritto di appartenervi, ovvero i cosiddetti "estranei alla comunità" - un'espressione che già di per sé li emarginava -, in prima fila gli ebrei. In ciò l'antisemitismo ebbe un ruolo decisivo ${ }^{15}$. «Può essere cittadino solo il compagno del popolo. Può essere compagno del popolo solo chi è di sangue tedesco, indipendentemente dalla confessione. Nessun ebreo quindi può essere compagno del popolo», stabiliva in modo chiaro e univoco il programma della Nationalsozialistische Deutsche Arbeiterpartei (Nsdap) del 1920.

La comunità era «l'idolo del secolo», notò già allora giustamente l'acuto osservatore Helmulth Plessner ${ }^{16}$. Dal XIX secolo il concetto di «comunità» costituiva il polo opposto del concetto di «società», facendosi espressione della critica alla rapidissima dinamizzazione e pluralizzazione dei rapporti sociali derivante dall'industrializzazione, dalla secolarizzazione, dall'orientamento verso il mercato e dal liberalismo politico. «La nostalgia di comunità nasce sempre dalla reazione contro un presente percepito come negativo. Così la realtà di questi modelli di comunità non va ricercata nel passato al quale questi di solito si riferiscono, ma nel presente» ${ }^{17}$. Si insiste sulla «comunità» sempre nella prospettiva della «società» moderna; essa è una parte costitutiva dell'auto-rassicurante "discorso della crisi" della modernità. Nessuno ha dato espressione scritta più eloquente a questa contrapposizione di Ferdinand Tönnies, il cui libro Gemeinschaft und Gesellschaft, apparso nel 1887, colpì il nervo del suo tempo e da allora in poi influenzò la discussione pubblica.

367; cfr. anche M. Wildt, Die Ungleichheit des Volkes. "Volksgemeinschaft" in der politischen Kommunikation der Weimarer Republik, in M. Wildt-F. Bajohr, Volksgemeinschaft cit., pp. 24-40.

15 Questo elemento è stato messo in evidenza precocemente da A. Barkai, The German Volksgemeinschaft from the Persecution of the Jews to the "Final Solution", in M. Burleigh (hrsg.), Confronting the Nazi Past. New Debates on Modern German History, Collins \& Brown, London 1996, pp. 84-97.

16 H. Plessner, Grenzen der Gemeinschaft. Eine Kritik des sozialen Radikalismus, (1924) Suhrkamp, Frankfurt am Main 2002, p. 28.

17 G. Raulet, Die Modernität der "Gemeinschaft”, in M. Brumlik-H. Brunkhorst (hrsg.), Gemeinschaft und Gerechtigkeit, Fischer, Frankfurt am Main 1993, p. 73. 
Nel concetto di «comunità» confluivano speranze di superamento dell'alienazione sia in senso rivoluzionario che restauratore. Quest'ambivalenza tra il ripristinare ciò che sembrava perduto e il realizzare nel futuro l'ordine sociale che appariva auspicabile era inerente fin dall'inizio al concetto di «comunità». Per questo si fraintenderebbe il concetto di Volksgemeinschaft se lo si considerasse la descrizione di una società realmente esistente. La forza politica dell'evocazione della Volksgemeinschaft non stava nella constatazione di una realtà di fatto quanto nella promessa, nella mobilitazione.

Eppure questo concetto è stato considerato a lungo dagli storici una mera formula propagandistica, la cui presunta efficacia, si riteneva, era suffragata principalmente da testimonianze di provenienza nazionalsocialista. «Cosa ci fa pensare», chiedeva Heinrich August Winkler nel 1977, «che sia giusto prendere per veri gli slogan politico-sociali dei nazionalsocialisti?»18. Nel contesto sociale degli anni ' 70 , in cui la maggioranza dei contemporanei aveva avuto esperienza diretta del nazionalsocialismo e spesso legava ricordi positivi alla Volksgemeinschaft, gli storici sociali cercavano sulla base di parametri socio-statistici di svelarne la natura di costrutto propagandistico lontano dalla realtà, anche per contrastare una possibile edulcorazione del nazionalsocialismo. Proprio in questo senso non molto tempo fa Hans Mommsen ha invitato a rinunciare in ambito analitico al concetto di Volksgemeinschaft che a suo parere avrebbe un'eccessiva coloritura propagandistica ${ }^{19}$.

Che i nazionalsocialisti strumentalizzassero questo concetto è fuori di dubbio. Non va però dimenticato che ampie fette della popolazione tedesca vedevano nella Volksgemeinschaft un obiettivo sociale auspicabile, anche se in parte vi legavano contenuti assai diversi. Eppure in questo modo divenivano possibili abboccamenti e convergenze con il regime, forme di consenso importanti per l'analisi del potere e della società nel nazionalsocialismo. Norbert Frei ha evidenziato l'importanza della "storia dell'esperienza" che, al di là delle persistenti diseguaglianze materiali e sociali, fece dell' «uguaglianza percepita» un significativo elemento di consenso ${ }^{20}$. Hans-Ulrich Wehler nella sua Gesellschaftsgeschichte ha evidenziato come il «fascino modernizzatore» e la carica mobilizzatrice legata alla Volksgemeinschaft abbiano generato una dinamica trasformatrice che contribuì in modo decisivo, specialmente nelle generazioni più giovani, alla legittimazione del regime - un'idea che in defi-

18 H.A. Winkler, Vom Mythos der Volksgemeinschaft cit., p. 485; cfr. l'argomentazione simile di H. Mommsen, Nationalsozialismus als vorgetäuschte Modernisierung, in W. Pehle (hrsg.), Der historische Ort des Nationalsozialismus, Fischer, Frankfurt am Main 1990, pp. 31-46; B. Weisbrod, Der Schein der Modernität. Zur Historisierung der "Volksgemeinschaft", in K. Rudolph-C. Wickert (hrsg.), Geschichte als Möglichkeit. Über die Chancen von Demokratie, Klartext-Verlag, Essen 1995, pp. 224-42.

${ }^{19} \mathrm{H}$. Mommsen, Forschungskontroversen zum Nationalsozialismus, «Aus Politik und Zeitgeschichte», 2007, n. 14-15, pp. 14-21.

${ }^{20}$ N. Frei, "Volksgemeinschaft" cit. 
nitiva si ritrova già in Martin Broszat ${ }^{21}$. «La questione della Volksgemeinschaft», ha scritto Norbert Frei, «ci porta al nocciolo del problema» 22 .

Contrariamente a quanto sostenuto da Götz Aly, secondo cui l'uguaglianza sarebbe stata un tratto distintivo della Volksgemeinschaft, la Volksgemeinschaft nazionalsocialista, la cui immagine propagandistica metteva al centro il superamento delle barriere sociali e l'unità del «popolo», era strutturata su nuove diseguaglianze. L'inclusione dei «compagni del popolo», infatti, si accompagnava a massicci processi di esclusione. Già nei primi anni '80 Detlev Peukert ha evidenziato questo dualismo di «compagni del popolo» ed «elementi estranei alla comunità» ${ }^{23}$. Gli stessi «compagni del popolo», d'altra parte, non erano cittadini cui venisse garantito il diritto alla libertà, non si trattava di uguaglianza di individui. Il centro della Volksgemeinschaft era piuttosto il «popolo» inteso come «corpo popolare» in senso organico-biologistico. «Tu non sei nulla, il tuo popolo è tutto», era il concetto centrale del regime. Non era tanto uno stato di quiete egualitaria a caratterizzare la Volksgemeinschaft, quanto la mobilitazione razzista, non il socialismo nazionale quanto l'accrescimento dell'efficienza a beneficio dello sviluppo del «corpo popolare» tedesco ${ }^{24}$.

Analizzare la Volksgemeinschaft quindi non vuol dire lasciarsi irretire da un concetto propagandistico del nazionalsocialismo o ignorare la realtà sociale, ma, al contrario, partire proprio dalla realtà sociale e scandagliare le dimensioni del consenso e del dissenso, della collaborazione e del rifiuto, della partecipazione e del "far finta di non vedere". Al centro dell'analisi vi è la prassi sociale, poiché proprio nel processo di costituzione della Volksgemeinschaft, nella «rifunzionalizzazione della società borghese tradizionale in una società dell'efficienza priva di vincoli morali» 25 - come l'ha definita una volta Martin Broszat - divengono visibili i tratti specifici della Volksgemeinschaft nazionalsocialista.

La rottura con lo stato di diritto della Repubblica tedesca avvenuta nel 1933 offrì a molte élite lo "spazio di realizzazione" che avevano sempre cercato. Finalmente al medico si apriva la possibilità di sanare il «corpo popolare», con la legge per la prevenzione della nascita di individui affetti da malattie ereditarie del luglio 1933 (Gesetz zur Verhütung erbkranken Nachwuchses), che per la prima volta in Germania consentiva la sterilizza-

${ }^{21}$ H.-U. Wehler, Deutsche Gesellschaftsgeschichte, v. 4, Vom Beginn des Ersten Weltkrieges bis zur Gründung der beiden deutschen Staaten 1914-1949, Beck, München 2003, p. 681; M. Broszat, Zur Struktur der NS-Massenbewegung, «Vierteljahrshefte für Zeitgeschichte», 1983 , n. 1 , pp. 52-76.

${ }^{22}$ N. Frei, "Volksgemeinschaft" cit., p. 128.

23 D. Peukert, Volksgenossen und Gemeinschaftsfremde. Anpassung, Ausmerze und Aufbegehren unter dem Nationalsozialismus, Bund Verlag, Köln 1982.

${ }^{24}$ U. Planert, Der dreifache Körper des Volkes: Sexualität, Biopolitik und die Wissenschaft vom Leben, «Geschichte und Gesellschaft», 2000, n. 4, pp. 539-76.

${ }^{25}$ M. Broszat, Struktur cit., p. 67. 
zione forzata contro la volontà del paziente. Solo nei primi tre anni i nuovi cosiddetti Tribunali per la salute ereditaria, cui oltre ad un magistrato appartenevano due medici, trattarono circa 224.000 casi, pronunciandosi nel 90\% dei casi (199.000) per la sterilizzazione. Funzionari della polizia criminale, finalmente non più limitati dal diritto, ritennero possibile creare una società senza criminali e di buon grado accolsero le premesse criminal-biologiche sulla cui base gruppi sociali definiti in senso razziale furono internati in campi di concentramento e marginalizzati in quanto «asociali». Funzionari dell'assistenza sociale suddivisero i loro assistiti non più in base ai loro bisogni, ma secondo la loro attitudine al lavoro, secondo il motto che chi non può lavorare non ha neanche bisogno di nutrirsi. E anche qui criteri razziali definirono presto la separazione degli individui "utili" alla Volksgemeinschaft dagli «elementi estranei alla comunità». Addirittura i funzionari del fisco con la Legge di riforma fiscale del 1934, il cui paragrafo 1 faceva dipendere l'applicazione delle norme fiscali dalla lealtà all'ideologia nazionalsocialista, potevano imporre a loro piacimento diseguaglianze - ossia un particolare aggravio fiscale, specialmente a carico degli ebrei - al posto dell'uguaglianza di tutte le cittadine e i cittadini davanti alla legge. Volksgemeinschaft significa dunque anche «auto-legittimazione», lo squadernarsi di un ventaglio di possibilità d'intervento rese possibili dal razzismo.

Il nazionalsocialismo offrì allo stesso tempo "spazi di realizzazione" della violenza. La violenza a sua volta consentì di rendere visibile la Volksgemeinschaft, poiché tramite campagne politiche furono tracciati i confini netti e invalicabili tra "noi" e i "nemici del popolo". L'antisemitismo costituì la Volksgemeinschaft nazionalsocialista, ne stimolò la radicalità e il potenziale distruttivo. Nella prassi politica locale ciò significò in primo luogo produrre distanza sociale, stigmatizzare ogni forma di solidarietà e di compassione per $\mathrm{i}$ perseguitati, isolare i vicini ebrei e dichiararli privi di diritti, fuorilegge. Il boicottaggio dei negozi ebrei non rimase confinato al $1^{\circ}$ aprile 1933 ma costituì proprio nelle province, nelle cittadine e nei villaggi un campo di azione efficace contro gli ebrei. Con il boicottaggio fu possibile sperimentare diverse forme di agitazione, dai manifesti esposti in pubblico, ai picchetti davanti ai negozi, al semplice incitamento ai clienti a non entrare nel negozio, fino agli insulti e all'uso della violenza ${ }^{26}$.

Accanto alle azioni di boicottaggio crebbero nell'estate del 1935 - quindi prima delle Leggi di Norimberga, promulgate a settembre - anche campagne contro le relazioni amorose tra ebrei e non ebrei, considerate «corruzione della razza». Ovunque nel Reich furono mosse simili accuse che si associavano sempre ad aggressivi attacchi pubblici sui giornali, tramite volantini o manifestazioni, durante le quali i presunti «corruttori della razza» venivano trascinati con la forza per le vie della città. Se si guardano le foto di quei cortei,

${ }^{26}$ Cfr. H. Ahlheim, "Deutsche, kauft nicht bei Juden!”. Antisemitismus und politischer Boykott in Deutschland 1924 bis 1935, Wallstein, Göttingen 2011. 
che avevano luogo in pieno giorno e in pubblico, salta agli occhi la folla che li accompagnava: donne, bambini, ragazzi seguono il corteo, ridono, prendono in giro, insultano, sputano sulle vittime. I curiosi e i passanti, quali che fossero le loro intime convinzioni rispetto all'accaduto, costituivano un elemento irrinunciabile di queste azioni che avvenivano in pubblico proprio per trasformare profondamente l'opinione pubblica ${ }^{27}$. Il regime nazionalsocialista socializzò la violenza, facendovi partecipare i «compagni del popolo». Ogni azione violenta cancellava linee di confine e, proprio perché poteva avvenire senza che la violazione del diritto fosse perseguita, trasformava l'ordine aprendo in esso nuove possibilità di intervento, non date prima di allora.

Se si adotta questa prospettiva individui apparentemente dipendenti divengono attori, sono nello stesso tempo sia soggetti che oggetti, sperimentano l'impotenza, la dipendenza da autorità, poteri e costrizioni economiche ma nello stesso tempo modificano queste esperienze in "modalità di appropriazione" specifiche, differenti e addirittura contraddittorie, possono addirittura farle sfociare in forme di renitenza. Allo stesso modo anche le esperienze di gioia, partecipazione e benessere possono essere legate a repressione, sfruttamento e dominio. I membri della Volksgemeinschaft tedesca durante la seconda guerra mondiale potevano impiegare come schiavi nelle loro case o fabbriche lavoratrici e lavoratori coatti dalle regioni occupate e sperimentare una sensazione di potere e superiorità.

Un uso produttivo del concetto di Volksgemeinschaft, dunque, richiede che lo si intenda in senso prasseologico, cioè non presupponendo la Volksgemeinschaft come un dato ma analizzando le pratiche della sua produzione, in poche parole: la sua socializzazione. Al centro di una simile prospettiva - possiamo argomentare così seguendo l'approccio di Alf Lüdtke - ci sono le «forme in cui gli uomini si sono "appropriati" del mondo e nel far ciò lo hanno anche continuamente modificato» ${ }^{28}$. Questo mondo e con esso le condizioni esterne sono dati ma nello stesso tempo anche prodotti, si rivelano quindi ricchi di significati e complessi, aperti a opzioni di intervento sia individuali che collettive. «Individui e gruppi plasmano le loro modalità di percezione e di azione non al di là, ma dentro e attraverso le relazioni sociali» ${ }^{29}$. Gli uomini non seguono semplicemente i codici, le rappresentazioni dei significati e della realtà che trovano già dati, ma utilizzano immagini, parole e pratiche per orientarsi. Le modificano, si scontrano con la rigidità, l'inflessibilità delle cose e degli uomini e così trasformano sia le cose che i rapporti sociali.

In questa prospettiva analitica la Volksgemeinschaft non costituisce una formazione sociale statica, ma andrebbe piuttosto analizzata in quanto prassi

27 Cfr. M. Wildt, Volksgemeinschaft als Selbstermächtigung. Gewalt gegen Juden in der deutschen Provinz 1919 bis 1939, Hamburger Edition, Hamburg 2007.

28 A. Lüdtke, Einleitung: Was ist und wer treibt Alltagsgeschichte?, in Id. (hrsg.), Alltagsgeschichte. Zur Rekonstruktion historischer Erfahrungen und Lebensweisen, Campus Verlag, Frankfurt am Main, 1989, p. 12.

${ }^{29}$ Ivi, p. 13. 
sociale. Non coglie nel segno, pertanto, la critica mossa da Ian Kershaw in un suo intelligente saggio, che offre un bilancio del dibattito in corso, secondo cui «il concetto di Volksgemeinschaft suggerisce il completo predominio di una sola identità» 30 e per questo rischierebbe di sottovalutare o addirittura oscurare le crepe nel consenso. Se si deve analizzare la Volksgemeinschaft come prassi sociale, allora va considerata proprio la molteplicità dei comportamenti, la collaborazione così come la presa di distanza, la disponibilità così come l'avversione, l'adattamento così come l'entusiasmo, il distanziarsi così come il «lavorare incontro al Führer». Bisogna, dunque, infrangere proprio la suggestione di omogeneità che proviene dalla Volksgemeinschaft.

Diversamente da precedenti ricerche che indagavano la «resistenza» e la «renitenza» oppure ritenevano la triade «persecutori-vittime-spettatori» un modello esauriente dell'azione sociale, qui è centrale una concezione della storia politica che non contrappone più stato e società. La Volksgemeinschaft richiama l'attenzione sul fatto che il politico non nasce solo nello stato ma anche nella società e proprio da essa. Ci fa capire che gli ordinamenti politici possono essere fondati nel sociale, nelle esperienze e nelle aspettative sociali. Lo sguardo scientifico sulla costituzione e sulla trasformazione del politico non dovrebbe dunque rivolgersi solo al livello dello stato ma contemporaneamente anche ai confini, ai meccanismi e ai mezzi storicamente mutevoli di uno spazio comunicativo politico strutturato in forme discorsive e simboliche $^{31}$. Il nuovo approccio al concetto di Volksgemeinschaft mostra quindi anche un mutamento di paradigma epistemologico nella storiografia sul nazionalsocialismo verso una storia culturale del politico.

\section{Hans Mommens, Il mito della Volksgemeinschaft}

Da quando il sociologo statunitense Seymour Martin Lipset formulò l'ipotesi secondo cui il nazionalsocialismo andrebbe definito come «estremismo del centro», la questione di quanto fosse elevata la partecipazione del ceto medio borghese all'ascesa della Nsdap come movimento di massa e del suo ruolo nella fase del regime è divenuta centrale nella contemporaneistica. Contro la tesi di Lipset la ricerca elettorale ha mostrato che una fetta non irrilevante degli elettori del nazionalsocialismo apparteneva alla classe lavoratrice e che quindi una semplice associazione tra Nsdap e ceto medio non è corretta. Nondimeno vi era una precaria relazione reciproca tra una borghesia che aveva perso il proprio riferimento politico e la Nsdap. È indubbio che nelle elezioni tenutesi dal settembre 1930 al marzo 1933 il ceto medio bor-

${ }^{30}$ I. Kershaw, "Volksgemeinschaft" cit., p. 12; cfr. anche la mia replica (M. Wildt, "Volksgemeinschaft" cit.).

${ }^{31}$ Cfr. U. Frevert-H.G. Haupt (hrsg), Neue Politikgeschichte. Perspektiven einer historischen Politikforschung, Campus Verlag, Frankfurt am Main 2005. 
ghese tra gli elettori della Nsdap fosse sovra-proporzionato. Nella crisi della tarda Repubblica di Weimar la borghesia protestante, in particolare, mostrò una spiccata tendenza a dare i propri voti a Hitler e a sciogliere il proprio legame con i partiti borghesi.

Questo dato di primo acchito sembra essere in contraddizione con l'atteggiamento di Hitler che, a causa della sua provenienza e della sua concezione politica, si presentava sempre come un nemico della borghesia e manifestava continuamente il suo disprezzo verso di essa. Di fatto era sua intenzione eliminare le forme politiche e sociali create dalla borghesia, condannate come «liberali». La propaganda nazionalsocialista contrapponeva all'universo valoriale borghese lo slogan della Volksgemeinschaft, che mirava a eliminare il conflitto tra classe lavoratrice e ceto medio borghese e a stabilire l'unità della nazione su base volontaria al di là di barriere cetuali e di differenze di reddito. In un'intervista con Hans Jost del gennaio 1934 Hitler descrisse l'auspicata Volksgemeinschaft come «comunità di tutto il lavoro produttivo, cioè unità degli interessi vitali, cioè superamento della borghesia privata e della massa organizzata su base meccanico-sindacale» ${ }^{32}$. Nonostante questa battaglia contro l'individualismo borghese, la promessa della propaganda nazionalsocialista di superare la lotta di classe raccolse unanimi simpatie proprio nell'alta borghesia.

Hitler usava presentarsi come un operaio e vantare una provenienza, in realtà inesistente, dalla classe operaia ma rivendicava alla Nsdap la rappresentanza di tutti i ceti popolari. Nel suo discorso al Lustgarten di Berlino del $1^{\circ}$ maggio 1937 egli sottolineò che la guida della nazione era stata assunta da «tedeschi di ogni strato sociale», tra questi anche «ex-borghesi» ${ }^{33}$. Il dittatore si espresse però anche in seguito contro ciò che percepiva come stile di vita borghese. Questo disprezzo della borghesia era condiviso dai suoi satrapi, la maggior parte dei quali erano arrampicatori sociali falliti o individui che avevano interrotto una carriera borghese. Il loro spregio della borghesia comunque non impedì loro di abitare lussuose ville di gusto borghese, perlopiù con aranciera, che spesso erano state proprietà di ebrei. Non poteva essere più grande il contrasto con l'immagine ideale del «compagno del popolo» nutrito dalla propaganda nazionalsocialista, che si distingueva per modestia, parsimonia, abnegazione e laboriosità.

Tanto più paradossale appare il fatto che la Nsdap, nelle elezioni decisive che ebbero luogo dal 1931 in poi, fu appoggiata in primo luogo dal ceto medio borghese. A fronte di ciò la sua base agraria, a cui doveva la schiacciante vittoria nelle elezioni del settembre 1930, cominciò a sgretolarsi già l'anno dopo e il partito, ad eccezione delle regioni a est dell'Elba, era sottorappresentato nelle campagne. Gli spettacolari successi elettorali della Nsdap erano

32 Cit. in R. Evans, Das Dritte Reich, v. 2, Diktatur, t. 2, Dva, München 2006, p. 603 (ed. or. London 2005, ed. it. Milano 2010).

${ }_{33}$ Passi del discorso in M. Domarus, Hitler. Reden und Proklamationen 1922-1945, v. 1, t. 2, Süddeutscher Verlag, München 1965, p. 690. 
dovuti, non da ultimo, all'infiltrazione nelle strutture sociali su cui poggiavano i partiti borghesi, che andavano da associazioni professionali, associazioni locali con carattere marcatamente politico e club sportivi, ad associazioni paramilitari. A ragione, dunque, lo storico americano Rudy Koshar nel suo studio su Marburgo ha evidenziato che proprio i risentimenti contro lo «stato dei partiti» di Weimar diffusi nel ceto medio prepararono il terreno all'ascesa della Nsdap ${ }^{34}$. Di fatto i nazisti riuscirono a penetrare nell'associazionismo borghese e a strumentalizzarlo per le loro ininterrotte campagne elettorali.

L'ostilità ai partiti del centro borghese e la sua crescente insofferenza per la politica negli anni di crisi della Repubblica furono utilizzate efficacemente dalla propaganda nazionalsocialista, che seppe usare con scaltrezza la promessa di superare la dittatura dei partiti esistita fin allora. «Mi sono posto uno scopo», spiegò Hitler nel suo spettacolare discorso elettorale a Eberswalde del 27 luglio 1931, «cioè spazzare via dalla Germania i trenta partiti oggi esistenti», e continuò, «i partiti scompariranno e dopo di essi tornerà ad esserci di nuovo il nostro popolo. E noi non vogliamo essere espressione di una professione, di una classe, di un ceto, di una confessione o di un paese» ${ }^{35}$. Questa evocazione hitleriana di una Volksgemeinschaft che avrebbe superato i contrasti interni incontrò la travolgente approvazione proprio degli elettori borghesi e suscitò un ampio entusiasmo che prese il sopravvento sui divergenti interessi politici e sulle differenze il classe. Così la Nsdap si rivolse in particolare alle nostalgie della borghesia.

La forza d'attrazione della parola d'ordine Volksgemeinschaft, che come ha scritto Norbert Frei liberò un «sentimento di uguaglianza sociale» ${ }^{36}$, mise in ombra le tensioni sociali esistenti e suscitò nel ceto medio speranze di superamento della critica situazione economica. Le promesse sociali del regime non trovarono però realizzazione. Il superamento del contrasto tra capitale $\mathrm{e}$ lavoro si limitò a gesti dimostrativi per l'opinione pubblica come la partecipazione degli imprenditori alle manifestazioni del $1^{\circ}$ maggio, le esigue riforme sociali come l'introduzione di un tetto massimo per i dividendi, i programmi cosmetici delle organizzazioni «Forza tramite il lavoro» (Kraft durch Freude) e «Bellezza del lavoro» (Schönheit der Arbeit), la partecipazione delle classi elevate ad azioni di solidarietà come il «Giorno della minestra» (Eintopfessen) e il «Soccorso invernale» (Winterhilfe).

Al contrario di quanto sostiene un recente filone storiografico che sottolinea i presunti successi della politica della Volksgemeinschaft, nei gruppi sociali interessati si diffuse presto una forte disillusione. Questo processo iniziò già nel 1934, quando Goebbels cercò di frenarlo con la sua «Campagna contro il disfattismo» e nel ceto medio toccò il culmine nel 1936. Per questo nei

${ }^{34}$ R. Koshar, Social Life, Local Politics and Nazism- Marburg 1880-1935, Universtity of North Carolina Press, Chapel Hill 1996, pp. 284 ss.

35 «Geschichte in Wissenschaft und Unterricht», 1959, n. 10, pp. 215-66.

${ }^{36}$ N. Frei, "Volksgemeinschaft" cit., p. 122. 
ceti medi l'euforia con cui inizialmente avevano salutato il gabinetto di Hitler si spense sempre più. Se fino ad allora la tradizionale frammentazione della borghesia in una molteplicità di associazioni e circoli giù giù fino ai caffè e la loro tipica mentalità apolitica avevano favorito la penetrazione nazionalsocialista, alla lunga esse si rivelarono più che altro un elemento di disturbo. Queste infatti si sottraevano al controllo dell'apparato del partito e divennero nemiche del patronaggio nel pubblico impiego da parte della Nsdap, in particolare dei favoritismi nei confronti dei «vecchi compagni di lotta».

Nella misura in cui la dinamica della ripresa economica, parallela all'estromissione di comunisti e socialdemocratici, tendeva a rallentare e le promesse di riforma generale della società non venivano realizzate, la politica di allineamento dei partiti si scontrò a livello comunale con una crescente opposizione. La nazificazione di quasi tutte le associazioni civili da parte della Nsdap, l'imposizione del Führerprinzip al posto delle elezioni interne usuali fino allora, il monopolio della Deutsche Arbeitsfront (Fronte del lavoro tedesco) fecero sì che le posizioni dirigenziali fossero assunte da persone privilegiate per la loro appartenenza al partito, che infransero norme di comportamento e procedure consolidate e marginalizzarono le élite tradizionali, che per lo più erano entrate nel partito. In questo modo l'infrastruttura borghese fu indirettamente politicizzata, anche se da questo processo furono esentate le associazioni ricreative del tutto neutrali. Questo fece sì che diminuisse l'interesse per queste associazioni civiche che godevano di una lunga tradizione e determinò una loro perdita di rappresentatività. Ciò è evidente se si considera l'esempio delle associazioni civiche di Amburgo una volta assai influenti, come sottolinea Frank Bajohr ${ }^{37}$. Influenti notabili si ritirarono a vita privata e lasciarono la politica locale nelle mani dei rampanti attivisti della Nsdap. Così $\mathrm{i}$ «milieu socio-morali» esistenti furono in larga misura distrutti.

Come sottolinea Richard Evans, il regime nazionalsocialista non portò maggiore uguaglianza sociale, sebbene questa fosse simulata senza posa dalla propaganda della Volksgemeinschaft ed esercitasse un'attrazione non irrilevante come proiezione nel futuro. Le differenze di classe rimasero vive come non mai. Contemporaneamente la Nsdap perse sempre più il proprio dinamismo. «Feste e celebrazioni divennero rituali vuoti a cui la gente partecipava più per paura che per convinzione». Considerando le condizioni della città di Northheim, Evans sottolinea: «Il gruppo locale della Nsdap dovette accontentarsi che le persone si adattassero e che pronunciassero vuote dichiarazioni di fedeltà» ${ }^{38}$.

Quest'umore diffuso tra la popolazione, che si manifestò già a metà degli anni '30, solo in rari casi si concretò in una vera opposizione. La mancanza

${ }^{37}$ F. Bajohr, Die Zustimmungsdiktatur. Grundzüge nationalsozialistischer Herrschaft in Hamburg, in Forschungsstelle für Zeitgeschichte (hrsg.), Hamburg im "Dritten Reich", Wallstein, Göttingen 2005, pp. 111 ss.

${ }^{38}$ R. Evans, Das Dritte Reich cit., p. 601. 
di una chiara divisione di ruoli in politica interna rendeva impossibile attribuire responsabilità per gli evidenti fallimenti del regime. Ciò ebbe come conseguenza una cristallizzazione della critica sui funzionari locali, che non si concretizzò in un generale rifiuto del sistema. Allo stesso tempo il bisogno di stabilità economica e politica paralizzò qualsiasi impulso all'opposizione e ne derivò una forte tensione intergenerazionale.

La ripresa economica determinata soprattutto dal riarmo fece sì che parti non irrilevanti delle generazioni più giovani proprio del ceto medio sperimentassero una rapida ascesa sociale o che acquisissero prestigio anche solo simbolico, in particolare nell'apparato del partito, ma anche in altri settori della burocrazia del regime in espansione, nell'impero delle SS, nel Fronte del lavoro tedesco e nella Wehrmacht. Indirettamente ciò interessò anche la classe operaia industriale, che inaspettatamente assorbì milioni di lavoratori coatti e di prigionieri di guerra. Mentre il boom economico determinato dal riarmo procurò ai più giovani ottime possibilità di ascesa e mentre questi si fecero abbagliare da apparenti successi e progressi, nelle file dei "guglielmini", come li si chiamava allora, si diffuse più che altro un attendismo scettico.

È dubbio comunque che questa formazione sociale nascente si possa definire una «società egualitaria dell'efficienza», come suggerisce Hans Ulrich Wehler, proprio in considerazione degli incisivi meccanismi di selezione e dei criteri di classificazione politici e razziali39. Allo stesso modo è assai dubbio che la Volksgemeinschaft esaltata non solo dalla propaganda, ma anche dalla legislazione corrispondesse alla realtà sociale e politica del regime. Ciò vale ancor di più per l'idea che la coesione interna della nazione fosse rafforzata dall" "esclusione" di ebrei e di elementi estranei alla comunità e che addirittura sia stata intensificata da aggressioni violente contro concittadini ebrei e azioni pubbliche contro «corruttori della razza». Certamente vi furono effetti di questo tipo nel nucleo radicale del movimento nazionalsocialista, ma difficilmente essi interessarono ampi settori della popolazione, che più che altro, come ben sappiamo oggi, rifiutavano atti di violenza contro gli ebrei e - in occasione della «notte dei cristalli» - si tennero lontani da aggressioni. Pertanto la tesi di Michael Wildt secondo cui la persecuzione degli ebrei fu «lo strumento politico essenziale della distruzione dello Staatsvolk e della creazione della Volksgemeinschaft», distorce del tutto la realtà storica ${ }^{40}$.

È incontestabile che l'ideologia nazionalsocialista della Volksgemeinschaft mirasse alla costituzione di «una società ideologicamente omogenea, socialmente allineata, orientata all'efficienza e strutturata in modo gerarchico», cosa che implicava anche l'«eliminazione» dei presunti «anormali», come l'ha definita Detlev Peukert ${ }^{41}$. È anche fuori discussione che soprattutto nel ceto medio vi fu una forte predisposizione ad accogliere queste idee. Ma altra co-

\footnotetext{
${ }^{39}$ H.-U. Wehler, Deutsche Gesellschaftsgeschichte cit., v. 4, p. 767.

${ }^{40}$ Cfr. M. Wildt, Volksgemeinschaft als Selbstermächtigung cit., p. 361.

${ }^{41}$ D. Peukert, Volksgenossen und Gemeinschaftsfremde cit.
} 
sa è vedere nella «creazione della Volksgemeinschaft» una strategia realistica che avrebbe trasformato tramite l'utilizzo della violenza, in particolare di quella antisemita quotidiana - come sostiene Michael Wildt - «la nazione tedesca in una Volksgemeinschaft aggressiva e razzista» 42.

La doppia tesi secondo cui da un lato l'obiettivo dell'accrescimento del potere, dall'altro la strategia d'integrazione sociale erano legati alla permanente produzione di violenza, è assai problematica. Essa attribuisce alla dirigenza nazionalsocialista una strategia razionale ed efficace di allocazione del potere che non è mai esistita in modo così netto. Nella fase del regime l'esercizio della violenza contro il nemico politico fu usato per impressionare gli elettori borghesi con una dimostrazione di potere. Ma l'ipotesi secondo cui le aggressioni violente contro gli ebrei siano servite all'obiettivo strategico dell'allocazione di potere e allo stesso tempo all'integrazione sociale è veramente eccessiva. Questa interpretazione attribuisce alla politica nazionalsocialista una consistenza concettuale che mal si adatta alla sua prassi politica reattiva e non considera le forze ciecamente ideologiche che condussero alla liberazione di energia violenta. Il carattere terrorista del nazionalsocialismo derivava dalla sua "visione del mondo", ma venne realizzato nella misura in cui cadevano le sanzioni contro l'esercizio di violenza arbitraria. Questo meccanismo difficilmente può essere interpretato come un elemento intenzionale della sindrome della Volksgemeinschaft. Nel nostro contesto va considerata soprattutto l'ipotesi secondo cui le azioni violente avrebbero condotto a una specifica trasformazione della nazione tedesca. Questa tesi implica che il regime sia riuscito a creare un ampio consenso e un "seguito" escludendo con successo gli «elementi estranei alla comunità» e gli ebrei. Certo, sia la propaganda che Hitler lo affermarono senza posa. Ma la realtà era diversa.

A parte poche eccezioni, non vi furono significativi fenomeni di opposizione nel ceto medio. In questo senso il regime non costituì una «società strutturata a nicchie» (Frank Bajohr). La penetrazione ideologica fu, anzi, forte in tutti gli ambiti sociali e vi furono solo poche eccezioni, come gli esponenti della proprietà terriera nobiliare e le chiese. D'altra parte vi furono diverse forme di lealtà. Il culto del Führer diffuso nel ceto medio non escludeva critiche alla Nsdap e ai suoi metodi terroristici. Dei crimini, di cui si aveva coscienza, e delle distorsioni sociali nel regime nazionalsocialista furono accusati solo i satrapi secondo la formula: «se lo sapesse il Führer». Infine l'approvazione iniziale del regime, come abbiamo detto, già a metà degli anni '30 fece posto anche in ambito borghese a una crescente disillusione.

Certo, i successi di Hitler in politica estera, che non furono messi in relazione con le condizioni interne, rafforzarono le simpatie per il Führer, che raggiunsero l'acme dopo la campagna contro la Francia. Queste però crollarono al più tardi quando divenne chiaro che la campagna di Russia non sarebbe terminata nell'autunno del 1941 e che la guerra sarebbe durata un se-

\footnotetext{
${ }^{42}$ M. Wildt, Volksgemeinschaft als Selbstermächtigung cit, p. 13.
} 
condo inverno. Hitler allora non fu più visto come il garante della pace, un'immagine che prima di allora aveva contribuito decisamente alla sua popolarità. Fino alla fine del 1944 rimase in parti della popolazione una cieca fiducia che Hitler avrebbe comunque saputo gestire la crisi.

Dal 1941 gli slogan della Volksgemeinschaft diffusi da Goebbels, al contrario, furono accolti dall'opinione pubblica tedesca in modo sempre più critico. Ciò che induceva il popolo a sostenere ancora il regime era la convinzione che in guerra, a maggior ragione quando incombeva il pericolo bolscevico, bisognasse essere fedeli alla nazione. All'interno la lealtà della popolazione fu sempre più logorata dagli interventi nella società civile dovuti alla guerra, che colpirono proprio i ceti borghesi - nel settore dell'istruzione, in quelli produttivo e culturale. Perciò concetti come quelli che circolano negli ultimi anni - «dittatura del consenso» (Frank Bajor) o «stato popolare» (Götz Aly) ${ }^{43}$ - sono piuttosto fuorvianti, per quanto non si possa dubitare del fatto che il regime nazionalsocialista attrasse inizialmente le simpatie di parti significative della popolazione, anche con la proclamazione della Volksgemeinschaft.

Lo stesso Wildt esita ad applicare il discorso della Volksgemeinschaft agli ultimi anni di guerra. È problematico, infatti, interpretare la resistenza di parti della popolazione e dei soldati della Wehrmacht come espressione della Volksgemeinschaft e vedervi abnegazione «per il popolo e la patria». Già solo i necrologi per i caduti nei giornali evidenziano questa trasformazione, sebbene la propaganda non volesse rendersene conto. Alcuni storici ignorano il semplice fatto che i soldati tedeschi non avevano alternativa all'obbedienza agli ordini dei propri superiori. Lo stesso vale per tutta la popolazione. Gli squadroni della morte delle SS e i tribunali speciali delle ultime settimane di guerra sono in questo senso assai eloquenti.

\section{Ulrich Herbert, Echi della Volksgemeinschaft}

Già a partire dal volgere del '900 il concetto di Volksgemeinschaft era stato centrale nella critica alla modernità, trasversalmente agli schieramenti politici. In esso si coniugavano la critica alle contrapposizioni confessionali e all'effetto disgregatore delle classi della società industriale sulla nazione con il rifiuto del pluralismo, della cultura della modernità e della dinamizzazione dei rapporti sociali. Così come il termine ad esso affine di «alienazione», questo concetto non era affatto un privilegio delle destre, ma divenne un obiettivo di tutti gli schieramenti politici.

Inoltre non si trattava di un fenomeno solo tedesco. Ovunque in Europa le tensioni sociali erano fortemente cresciute dopo la prima guerra mondiale e quasi ovunque le divisioni interne determinate dalla contrapposizione di clas-

${ }^{43}$ F. Bajohr, Die Zustimmungsdiktatur cit., p. 111; G. Aly, Hitlers Volksstaat, Raub, Rassenkrieg und nationaler Sozialismus, Fischer, Frankfurt am Main 2005, pp. 88 ss. e passim. 
se furono deplorate come uno stato di cose innaturale. In Spagna così come in Italia, in Polonia, Ungheria e Jugoslavia così come in Grecia e Bulgaria si stabilirono regimi autoritari di destra, che postulavano il superamento delle spaccature interne e la creazione dell'unità nazionale, promettendo, se necessario, di imporla con la violenza. Si può addirittura parlare di un'egemonia di concetti nazionalistico-comunitari negli anni ' 30 in quasi tutta Europa, una reazione politico-ideologica all'ascesa del concetto di lotta di classe negli anni precedenti e successivi alla prima guerra mondiale. Ma anche nelle utopie delle sinistre radicali la rivoluzione avrebbe messo fine alla lotta di classe e avrebbe creato uno stato di uguaglianza senza conflitti.

In Germania queste tensioni erano particolarmente accentuate. Da un lato l'affermazione della moderna società industriale si era svolta in modo assai rapido. Dall'altro lato sia le contraddizioni nazionali che quelle sociali erano state acuite dalla sconfitta in guerra. Nell'idea di unità prima nazionale, poi völkisch si esprimeva il malcontento per il complicato gioco degli schieramenti, per l'associarsi e il dissociarsi degli interessi politici e sociali, per la faticosa ricerca di compromessi e per le coalizioni tipici di una società industriale caratterizzata da complessità e differenziazione.

Oltre a ciò però la Volksgemeinschaft era anche la portatrice del «sano sentimento popolare» contro l'estraneo in sé e per sé - una cifra del rifiuto della cultura di massa moderna e orientata in senso internazionale. I nazionalsocialisti accolsero questa richiesta e la radicalizzarono in modo estremo, da un lato tramite una forte riduzione di complessità, postulati di uguaglianza e prestazioni sociali, dall'altro tramite esclusione politica e razziale e integrazione negativa. Tutto questo riuscì in misura notevole finché fu accompagnato da successi economici e militari.

Vivere un periodo di stabilizzazione economica dopo 25 anni di crisi ininterrotte si rivelò l'esperienza più importante del periodo nazionalsocialista fino al 1940 circa. Anche chi non appoggiava politicamente i nazionalsocialisti apprezzava lo sviluppo economico, la rapida eliminazione della disoccupazione, la fine delle agitazioni sociali e se ne avvantaggiava, pur sapendo in quale modo era stata prodotta questa tranquillità. Ciò non significava che $\mathrm{i}$ tedeschi ora vivessero effettivamente meglio. I salari reali raggiunsero il livello dell'anteguerra solo nel 1938 e la vita in Germania, anche a confronto della Francia, della Gran Bretagna, degli Stati del Benelux, per non parlare degli Usa, continuava ad essere decisamente misera. Ma il miracolo economico nazionalsocialista consentì pur sempre di sperare in condizioni di stabilità, e a questa speranza era legata anche una grossa parte dei consensi di quella maggioranza dei tedeschi che nel 1932 avevano votato i nazionalsocialisti.

La seconda guerra mondiale però mise in discussione questi sogni di una vita migliore, che significavano soprattutto società dei consumi. Certo, ancora dopo lo scoppio della guerra la preoccupazione principale della maggioranza dei tedeschi era l'avanzamento personale e l'aspirazione a consolidare quella normalità finalmente raggiunta dopo decenni di privazioni con un po- 
sto di lavoro e un reddito fisso, e a non farsi distruggere dagli effetti collaterali della guerra. Nella maggior parte dei casi però senza successo.

Anche la dirigenza del regime riteneva molto importante tenere il più alto possibile 1" "umore" della popolazione e risparmiarle ogni aggravio. Ciò però non fu più possibile quando con le prime sconfitte ad est il numero delle perdite della Wehrmacht crebbe drammaticamente e gli attacchi aerei, prima sporadici poi regolari, resero almeno nelle città quasi impossibile una vita normale. Certo, nei primi due anni di guerra questi svantaggi furono in parte compensati dalle roboanti vittorie della Wehrmacht. In particolare quella contro la Francia produsse uno stato di ebbrezza nazionale che consolidò enormemente il legame tra popolo e dirigenza. Dalla metà del 1942 però l'umore della popolazione tedesca, soprattutto nelle grandi città minacciate dai bombardamenti, divenne sempre più intorpidimento e apatia. Il restringimento della percezione sociale all'immediato contesto della propria esistenza e il disinteresse crescente per tutti gli avvenimenti sociali erano le forme in cui tutto ciò si esprimeva. Così si sciolse sempre più il legame emotivo con il regime e anche con Hitler. Nella misura in cui si avvicinava la sconfitta i crimini del regime - di cui la popolazione sapeva o comunque sospettava qualcosa e quindi anche l'adesione al regime divennero sempre più un peso o addirittura una minaccia. Ciò accelerò il dissolversi del legame politico ed emotivo con il regime. Il consenso di gran parte della popolazione tedesca verso il Terzo Reich era legato ai suoi successi, sia militari che economici. Finiti questi, il regime perse la propria fonte di legittimazione.

La propaganda aveva tentato in sempre nuove varianti di dichiarare già compiuta la cancellazione delle differenze di classe tramite il comune riferimento alla Volksgemeinschaft. La struttura della società tedesca negli anni '30 era però rimasta decisamente stabile: la quota di ricchezza della classe lavoratrice rispetto alla società nel suo complesso si modificò appena; durante la guerra crebbe leggermente il numero di lavoratori; classe operaia e contadini erano comunque il $70 \%$ circa della società. La diseguaglianza sociale, che nella realtà persisteva e in parte si aggravava addirittura, fu compensata dal regime con una propaganda di tipo social-egualitario sempre più diffusa.

Eppure uno sguardo attento alla struttura della società nazionalsocialista rivela che, soprattutto durante la guerra, era sopraggiunta una dinamica significativa in questo rigido modello di appartenenza di classe. Circa dieci milioni di tedeschi furono richiamati nell'esercito durante la guerra e furono sostituiti da circa 8 milioni di lavoratori coatti stranieri e prigionieri di guerra. Contemporaneamente crebbe numericamente la forza lavoro maschile e femminile nell'industria, circa un terzo di tutti i salariati si trasferì durante la guerra in impianti che producevano per il riarmo. Un notevole numero di donne e giovani prestava servizio nella difesa antiaerea, come forze ausiliarie della Wehrmacht, nel servizio agricolo obbligatorio (Landjahr) e in quello del lavoro (Reichsarbeitsdienst). Il numero di persone direttamente o indirettamente attive per la guerra, senza un rapporto di lavoro formale, non può essere 
neanche fissato con precisione per l'ultimo anno del conflitto. Le stime si aggirano tra i 6 e i 7 milioni.

Si aggiunsero i profondi mutamenti sociali e spaziali, che interessarono anch'essi diversi milioni di persone, imposti dai bombardamenti, dal trasferimento delle fabbriche, dagli sfollamenti dei bambini e infine dalla fuga e dalle espulsioni. Questi processi accelerati sciolsero gli individui da legami fino ad allora molto stretti, appartenenze sociali, politiche, confessionali e in parte anche regionali. Al loro posto si formarono «comunità di destino», le cui sorti dipendevano da fattori geografici, militari, politici e non in ultimo dal caso.

A ciò erano connesse le esperienze di ascesa sociale fatte soprattutto durante la guerra, vuoi nel settore militare, vuoi nel partito o nelle organizzazioni di massa della Nsdap. Esperienze come il servizio militare, la prigionia di guerra, l'evacuazione o l'espulsione, nonostante il loro carattere collettivo, indicano soprattutto processi di singolarizzazione e individualizzazione e fecero sentire i loro effetti ancora nel dopoguerra. Così la stratificazione sociale della Bundesrepublik era già al suo inizio porosa e dalla fine degli anni ' 50 le nette appartenenze di classe e milieu persero di significato in modo prima lento, poi sempre più rapido. Non tanto omogeneizzazione e de-differenziazione nella Volksgemeinschaft, quanto piuttosto individualizzazione e pluralizzazione dei percorsi di vita caratterizzavano l'immagine della società tedesca alla fine della guerra. Allo stesso tempo divenne chiaro che le differenze tra poveri e ricchi, tra élite proprietarie e maggioranza della popolazione erano, nonostante tutte le affermazioni propagandistiche, addirittura cresciute.

La parola d'ordine della Volksgemeinschaft, in definitiva, perse forza incisiva anche perché, dalla presa del potere dei nazionalsocialisti, a fronte dei postulati di unità vi era una prassi del tutto opposta: la società nazionalsocialista, infatti, era costruita in misura notevole su relazioni personali, corruzione e privilegi. Nei primi anni della dittatura i cacciatori di poltrone e i carrieristi di partito erano visti come fenomeno collaterale di un rivolgimento politico. Al più tardi negli anni di guerra però l'abbattimento dei controlli politici e parlamentari, l'erosione delle limitazioni legali e i conflitti tra istituzioni concorrenti ebbero effetti sempre più catastrofici. Strutture istituzionali e vie legali trasparenti lasciarono il posto a un eldorado di informalità, che, se nella seconda metà del conflitto fu legittimato dalla necessità di decisioni rapide, portò, soprattutto ai livelli più bassi, a una lotta di tutti contro tutti. Dal 1942 cominciò a dominare il mercato nero: viveva meglio chi aveva contatti nelle regioni occupate, chi riusciva a mettere le mani su «beni ebraici», chi aveva qualcuno che intercedesse per lui presso le autorità e nell'apparato del partito. Chi era scaltro e privo di scrupoli, chi aveva "relazioni" e sapeva usare i trucchi giusti era tra i vincitori; gli altri dovevano cavarsela da soli. Al più tardi in quel periodo la parola d'ordine Volksgemeinschaft, propagata a gran voce, divenne una formula vuota su cui la gente ironizzava perché distorceva del tutto la realtà.

Anche chi non condivide l'accentuazione a volte eccessiva di questo concetto integratore völkisch, deve ammettere che il postulato nazionalsocialista 
della Volksgemeinschaft ebbe una certa efficacia nel consolidare la coesione interna dei tedeschi, nel legare ampie parti della società al regime e soprattutto nel far accettare in modo consenziente o solo passivo la politica della violenza del regime verso gli ebrei e le altre minoranze. In questo senso esso fu più di un mero strumento propagandistico per ingannare e corrompere. $\mathrm{E}$ allo stesso modo è chiaro che il consolidamento o addirittura la produzione di questa coesione interna tramite l'esclusione dei «nemici» - un tipico strumento di dominio nazionalsocialista - fu efficace in alcuni periodi. Allo stesso tempo però la sua efficacia dipendeva dall'esistenza di risultati comprovabili del regime e soprattutto dai suoi successi. In questo senso sono eloquenti le lamentele dei servizi segreti nazionalsocialisti sulle forti aspettative della popolazione nei confronti dei risultati concreti del regime che attraversano come un filo rosso tutti i rapporti fin dall'inizio della guerra. Inoltre il vantaggio di appartenere al gruppo dei non perseguitati, al più tardi quando la situazione di guerra nel 1941-42 cominciò a peggiorare rapidamente, divenne un peso e una minaccia per i singoli.

Alla fine era rimasto ben poco dei concetti e delle promesse d'integrazione del regime, vista l'atomizzazione della società tedesca nell'ultima fase della guerra, la lotta darwinista di tutti contro tutti e l'assenza di vie d'uscita in uno scenario di distruzione totale. Le amministrazioni militari alleate nelle loro relazioni descrivevano l'enorme disillusione, lo sbandamento e l'apatia che dominava tra i tedeschi. Così, solo alcuni elementi isolati delle esperienze fatte nel periodo nazionalsocialista poterono essere conservati nel lungo periodo: la breve esperienza della stabilità economica, l'ascesa sociale, lo scioglimento dei legami con il milieu e le strutture tradizionali; per una parte considerevole della popolazione, poi, andava annoverata anche la soppressione dei conflitti di classe tramite la distruzione del movimento operaio.

Il postulato della Volksgemeinschaft come principio di solidarietà nazionale e di appartenenza völkisch era già decisamente eroso dopo il 1945. Le aspettative dei tedeschi verso il futuro erano caratterizzate soprattutto dalla ricerca di sviluppo economico e di stabilità sociale. Le esperienze del periodo nazionalsocialista avevano ancora un'influenza nella misura in cui ci si ricordava della breve fase della ripresa economica negli anni precedenti alla guerra, che aveva nutrito grandi speranze ma poi aveva profondamente deluso. Nello stesso tempo si perpetuarono nella Bundesrepublik elementi della tradizione del comunitarismo nazionale come obiettivo sociale. Gli sforzi di risolvere le contraddizioni sociali in modo pacifico erano da un lato, nel loro significato anticomunista, un prodotto della guerra fredda. D'altra parte esse corrispondevano anche alla tendenza, osservabile nella Germania occidentale così come nella maggior parte dei paesi europei, ad appianare le contrapposizioni sociali non tramite il conflitto ma attraverso strutture corporative istituzionalizzate.

Eppure la categoria di comunità nazionale visse una certa rinascita nella forma della comunità delle vittime quando i tedeschi, nell'immediato dopoguerra e spesso ancora molto dopo, si sentirono vittime di violenza e di espul- 
sione, accusati collettivamente, discreditati moralmente di fronte al mondo e una semplice pedina degli Alleati: in ciò costituirono una comunità. Ma anche questo si rivelò essere solo un effetto transitorio. Tali reminiscenze persero significato nella misura in cui si costituì una società a più voci, aperta alle contraddizioni, che diversamente da quanto ci si aspettava si rivelò stabile e coronata dal successo. L'aver vissuto in una società di lupi durante la dittatura nazionalsocialista e la guerra aveva accresciuto il bisogno di regole, di incorruttibilità, dello stato di diritto, probabilmente più che di democrazia.

Se si tirano le somme di questi dati in parte contraddittori, l'incidenza della parola d'ordine nazionalsocialista della Volksgemeinschaft si rivela decisamente limitata. In ambito culturale già durante la guerra e in particolare dopo di essa si affermarono, rispetto a tutte le tendenze all'isolazionismo nazionale, la dinamica della cultura popolare occidentale e l'orientamento verso una società dei consumi. È innegabile che gli elementi che più caratterizzarono la Germania occidentale nei decenni del dopoguerra esistevano, tranne che per pochi aspetti, in tutte le società dell'Europa occidentale del tempo. Il mutamento della struttura sociale, l'appianamento dei confini di classe e dei ruoli di genere, la perdita di significato dei milieu socioculturali, la stabilità economica e la sicurezza sociale, la realizzazione di sistemi di sicurezza sociale, il rifiuto della lotta di classe a favore delle strutture corporative, l'affermarsi della società dei consumi così come le aspirazioni della critica della cultura a arginare la cultura di massa e a stabilire modelli di società tradizionali - tutti questi elementi si ritrovano con diverse sfumature nei Paesi Bassi e nella Svizzera così come in Italia, in Francia o nei paesi scandinavi e, seppur con significative differenze, in Gran Bretagna.

Tutto ciò relativizza il significato degli sviluppi nazionali rispetto all'accentuata path dependence del processo di modernizzazione nelle società industriali. Con ciò però va riesaminata anche la questione della forza plasmante e dell'efficacia della politica sociale del regime nazionalsocialista. Con il suo percorso di guerra e di sterminio essa ha sì messo a ferro e fuoco mezzo mondo, ma considerato il breve periodo di dominio di 12 anni la sua incidenza non fu così profonda da dare al processo di sviluppo della società industriale tedesca dopo il 1945 una forma sostanzialmente diversa da quella di altri paesi. Così la Volksgemeinschaft si rivelò il fugace sogno di un'unità nazionale che dopo il 1945 si volatilizzò.

(traduzione di Paolo Fonzi) 
Copyright $(\odot$ FrancoAngeli

N.B: Copia ad uso personale. È vietata la riproduzione (totale o parziale) dell'opera con qualsiasi mezzo effettuata e la sua messa a disposizione di terzi, sia in forma gratuita sia a pagamento. 


\title{
Combattenti solitari. Lavoratori tedeschi e ungheresi in epoca postcomunista
}

\author{
Eszter Bartha
}

Lonely fighters. German and Hungarian workers in post-communism period introduces the results of a life-history interview project conducted with workers and former workers of two large ex-socialist model factories, Carl Zeiss in Jena (East Germany) and Rábe in Győr (Hungary) between 2002 and 2004. The essay analyzes the post-socialist experience of the East German and Hungarian workers in three main dimensions: the experience of post-Fordist development in the factory; the subjective evaluation of the standard of living; interpersonal relations. Lastly, it examines examines the social and political attitudes of the workers in the mirror of their post-socialist experience. Hungarians had a more direct experience of peripheral development than the East Germans. While East Germany's greater success of integration into the capitalist world economy was accompanied by a change of mentality and the appearance of post-materialistic values, in Hungary nationalism seemed to be the only alternative to a capitalism, that disappointed and effectively impoverished many people. This explains the ambiguous evaluation of the socialist Kádár regime, in which the vision of greater social and material equality is confused with a longing for a strong state, order and an autocratic government.

Key words: Germany, Hungary, post-communism, Zeiss, Rábe, factory workers, oral history, transition studies, populism

Parole chiave: Germania, Ungheria, post comunismo, Zeiss, Rabe, operai, storia orale, studi sulla transizione, populismo

Fra il 2002 ed il 2004 ho raccolto, tramite interviste, 44 storie di vita di lavoratori ed ex-lavoratori di due ex-fabbriche socialiste modello, la Carl Zeiss a Jena in Germania est, e la Rába a Győr in Ungheria, che all'epoca del socialismo di stato impiegavano oltre 20.000 persone $^{1}$. Entrambi gli stabilimenti

\footnotetext{
${ }^{1}$ La ricerca è stata condotta con il sostegno di una borsa "János Bolyai" dell'Accademia ungherese delle scienze. In epoca comunista le due aziende erano considerare fabbriche modello. Nel 1846 l'imprenditore Carl Zeiss aveva fondato a Jena un laboratorio ottico e meccanico di precisione, ma il vero decollo dell'impresa si ebbe con la collaborazione del fisico e filantropo Ernst Abbe e di Otto Schott, un chimico specializzato in lenti ottiche di alta qualità.
}

«Passato e presente», a. XXXI (2013), n. 88 
sono sopravvissuti al cambio di regime riducendo drasticamente la manodopera: al momento dell'intervista, la Zeiss impiegava 3.000 persone, la Raba 4.500. Tutti gli intervistati avevano più di 38 anni e la maggioranza di essi apparteneva alla fascia d'età compresa fra i 40 e i 60 anni. In entrambi i gruppi uomini e donne erano distribuiti in maniera uniforme. Fra di loro vi erano membri dei disciolti partiti comunisti, leader di brigate del lavoro, capisquadra ed anche alcuni ingegneri, che avevano cominciato la loro carriera come operai ed erano saliti lungo la scala sociale partecipando ai programmi di formazione degli adulti fortemente incentivati dal partito in entrambi i paesi.

Questa ricerca di storia orale è nata da un progetto il cui obiettivo era analizzare i rapporti fra classe operaia e potere politico nella Ddr ed in Ungheria dalla fine degli anni '60 al collasso dei regimi comunisti. Tale progetto è stato poi sviluppato in direzione di una comparazione fra le esperienze postsocialiste dei lavoratori tedeschi orientali e di quelli ungheresi e fra le rispettive memorie dei regimi di Honecker e Kádár. La ricerca affronta inoltre la questione di come tali esperienze abbiano contribuito a plasmare la coscienza sociale e politica degli operai.

I partiti comunisti che dopo la seconda guerra mondiale andarono al potere in tutti i paesi dell'Europa orientale consideravano la grande massa degli operai industriali come la propria base sociale principale e concentrarono su di essi le loro politiche sociali. In un mio precedente studio, ho sostenuto che tale linea politica può essere utilizzata come chiave interpretativa per spiegare il collasso dei regimi ${ }^{2}$. Le «dittature del welfare» di Honecker e Kádár cercarono di conquistare i favori delle "masse" con la promessa di un continuo incremento dei livelli di consumo. Questa politica, inizialmente efficace, si

Imprenditori illuminati, i tre realizzarono generose politiche sociali che ebbero un ruolo chiave nel garantire all'azienda un duraturo successo e la dedizione degli addetti. Dopo la seconda guerra mondiale, una nuova fabbrica Carl Zeiss fu fondata a Oberkochen in Germania ovest, mentre la Carl Zeiss di Jena fu nazionalizzata. La rivalità dei due marchi fu uno dei simboli della competizione fra le due Germanie durante la guerra fredda. Con la riunificazione, la Carl Zeiss di Jena fu rilevata dalla Treuhand (Agenzia governativa tedesca che si occupò della privatizzazione delle imprese pubbliche dell'est) e privatizzata. Per una storia della Carl Zeiss di Jena in periodo comunista cfr. W. Mühlfriedel-E. Hellmuth, Carl Zeiss in Jena 1945-1990, Böhlau, Weimar 2004. La Fabbrica ungherese vagoni ferroviari e macchine da lavoro, nota come Rába Mvg, fu fondata nel 1896 a Győr, centro commerciale e amministrativo dell’Ungheria occidentale. La fabbrica esportava in Serbia, Romania, Italia ed Egitto e produsse anche carrozze per la metropolitana di Londra e la rete tramviaria di Anversa. Fu nazionalizzata nel 1946. Negli anni '60 l'ambizioso manager Ede Horváth, proveniente da una famiglia operaia e divenuto celebre come stakanovista, progettò un aumento della produzione di motori e assali e una diminuzione di quella di vagoni ferroviari. Nel dicembre 1989 Horváth, membro anche del comitato centrale del partito, si dimise su pressione del consiglio aziendale. Nel 1991 la fabbrica passò sotto il controllo dell'Agenzia Proprietà Statali (Spa) e nel 1997 fu privatizzata. Sulla storia della Rába cfr. Z. Tabiczky, A Magyar Vagon- és Gépgyár története, Győr 1977; E. Horváth, Én volnék a Vörös Báró? Szikra, Pécs 1990; A. Dusza, A birodalom végnapjai, Így láttam Horváth Edét, X-Meditor Kft, Győr 2003.

2 E. Bartha, Welfare Dictatorship, the Working Class and the Change of Regimes in East Germany and Hungary, «Europe-Asia Studies», 63 (2011), n. 9, pp. 1591-610. 
dimostrò sul lungo periodo dannosa per il regime, in quanto spostò a destra la coscienza politica della classe operaia ed escluse dalla sfera pubblica alternative di sinistra ${ }^{3}$.

La dittatura di Honecker nella Ddr fu decisamente più dura di quella di Kádár in Ungheria, soprannominata in Europa orientale «l'allegra caserma». Il ricorso alla repressione politica era più frequente ed era richiesta ai cittadini (in particolare agli iscritti al partito) una maggiore disciplina ideologica, laddove in Ungheria anche i membri del partito ed i segretari delle cellule di base potevano esprimere apertamente il loro scontento per le condizioni di vita fin dagli anni ' $80^{4}$. Inoltre, in Ungheria le difficoltà economiche costrinsero il governo a fare alcune concessioni al mercato che diedero vita ad un'economia parallela, informale e relativamente ampia, nella quale le persone potevano svolgere un secondo lavoro e ottenere guadagni extra ${ }^{5}$. Già a metà degli anni ' 80 il sociologo ungherese Erzsébet Szalai affermava che la partecipazione della classe operaia all'economia parallela stava in realtà sgretolando la coscienza di classe e contribuendo all'atomizzazione delle comunità operaie 6 .

L'esperienza immediata del cambio di regime fu differente nei due paesi. In Germania Est le dimostrazioni di massa segnarono la completa perdita di legittimazione del regime di Honecker, laddove in Ungheria l'Mszmp concordò con le opposizioni l'organizzazione di elezioni democratiche ${ }^{7}$. Per meglio confrontare queste due esperienze profondamente diverse di cambio di

${ }^{3}$ In Ungheria l'idea di un socialismo democratico senza il Mszmp (Magyar Szocialista Munkáspárt, Partito socialista ungherese dei lavoratori) venne incarnata più compiutamente dall'Unione per un'alternativa di sinistra (Baloldali Alternatíva Egyesület). Dopo il fallimento di tale progetto politico, la sua eredità intellettuale venne raccolta dal giornale «Eszmélet» (Consapevolezza), pubblicato nel 1989. L'intellettuale più noto a livello internazionale di questo circolo è Tamás Krausz.

${ }_{4}$ Sulla Ddr cfr. M. Fulbrook, Anatomy of a Dictatorship. Inside the Gdr, 1949-1989, Oxford UP, New York 1995. Sul caso ungherese cfr. J. Rainer, Bevezetés a kádárizmusba, L'Harmattan, Budapest 2011.

5 Alcuni autori considerano tale economia parallela un preludio al capitalismo. Cfr. Á. Róna-Tas, The Great Surprise of the Small Transformation: The Demise of Communism and the Rise of the Private Sector in Hungary, The University of Michigan, Ann Arbor 1997. Gli economisti ungheresi ritenevano tuttavia che tale economia informale restasse dipendente dallo stato: cfr. R.I. Gábor-P. Galasi, A “második” gazdaság: Tények és hipotézisek. Közgazdasági és Jogi Könyvkiadó, Budapest 1981.

${ }^{6}$ E. Szalai, Beszélgetések a gazdasági reformról, Pénzügykutató Intézet Kiadványai, Budapest 1986.

7 Il cambio di regime in Ungheria è stato definito «rivoluzione negoziata» o «rivoluzione costituzionale». Sulla storia politica dei negoziati fra il Mszmp edi nuovi partiti cfr. A. Bozóki (a cura di), A rendszerváltás forgatókönyve: kerekasztal-tárgyalások 1989-ben: alkotmányos forradalom: tanulmányok, Új Mandátum Könyvkiadó, Budapest 2000. Sulle radici storiche della transizione pacifica cfr. R.L. TEkés, Hungary's Negotiated Revolution: Economic Reform, Social Change, and Political Succession, 1957-1990, Cambridge UP, New York, 1996. Sulla Ddr cfr. C. Maier, Dissolution: The Crisis of Communism and the End of East Germany, Princeton UP, Princeton 1997. 
regime (nella Ddr i cittadini parteciparono attivamente, mentre in Ungheria ebbero la sensazione che i negoziati fossero un "affare" della nuova élite), sarà utile citare un'intervista svolta su un terreno "insolito" nella Germania est con un lavoratore della Zeiss (uno Zeissianer), che al tempo di Honecker era stato incarcerato per la sua attività di oppositore. Lasciata la Ddr nell'estate del 1989, aveva trovato lavoro a Monaco nel settore dei trasporti, poi perso in seguito a un incidente. Non essendo riuscito a trovarne un altro, al momento dell'intervista viveva in un ospizio per senzatetto. Così egli ricordava il passato socialista alla luce della sua esperienza nella nuova società capitalista:

Eravamo 50 nel gruppo [di opposizione]. Non facevamo grandi cose: pubblicavamo alcuni manifesti ed un giornale su cui scrivevamo che nella Ddr c'era repressione politica. Nel 1982 [gli uomini del partito] presero la cosa molto sul serio, io venni arrestato e feci sei mesi di prigione. Quando venni rilasciato, l'organizzazione si era dissolta e non aveva senso continuare. Non avevo alcun problema in fabbrica, guadagnavo bene. Ciò che non mi andava era che non potevo avere una mia opinione. Non potevi dire apertamente quello che pensavi perché eri costantemente spiato, anche al pub o dentro la fabbrica.

Qualsiasi cosa era considerata attività antinazionale e sovversiva. Era impossibile dire «State sbagliando» [al partito]. «Il Partito decide tutto, senza il partito l'erba non cresce e gli uomini non possono respirare» - questo era l'atteggiamento generale. Le persone volevano pensare da sole, dare suggerimenti, migliorare le cose - nessuno ascoltava. Il Partito ha sempre ragione, non dovevi pensare, dovevi solo lavorare. Volevano togliere alla gente la capacità di pensare. Bisognava solo fare il proprio lavoro e lasciare le cose serie ai dirigenti. Non vedo grandi cambiamenti in questo. Quelli che stanno al vertice non vogliono che la gente pensi. Oggi non vedo una grande differenza fra $i$ due sistemi, socialista e capitalista ${ }^{8}$.

La storia di Jan non rappresenta il percorso tipo degli operai della Germania est. La citazione, tuttavia, mostra una differenza cruciale fra le valutazioni soggettive delle due «dittature del welfare». In Germania est, nessuno, nemmeno Jan che aveva perso il lavoro e la casa nel nuovo regime, voleva il ritorno dello stato di Honecker. Nelle interviste ungheresi abbiamo trovato un'immagine più ambigua: l'aspirazione ad una maggiore uguaglianza sociale e materiale portava con sé il desiderio di uno stato più forte, d'ordine, e di un governo autoritario, che ridesse orgoglio alla nazione, proteggesse l'industria ungherese e migliorasse il livello di vita dei lavoratori - slogan, quest'ultimo, fra i più attraenti del regime di Kádár'.

\footnotetext{
${ }^{8}$ Intervista con Jan (52 anni), fino al 1989 operaio specializzato alla Zeiss, residente nel 2004 in un ospizio per senzatetto di Jena (corsivo mio).

${ }^{9}$ Per una prima analisi del caso ungherese cfr. E. Bartha, Munkások a munkásállam után. A változás etnográfiája egy volt szocialista "mintagyárban", in D. Némedi (a cura di), KötLEjelek. Az Eötvös Loránd Tudományegyetem Szociológiai Doktori Iskolájának Évkönyve, «Elte Szociológiai és Szociálpolitikai Intézet», Budapest 2003.
} 
Per spiegare le diverse valutazioni del cambio di regime e delle nuove democrazie è importante mettere in evidenza le differenze strutturali fra i due paesi, che modellarono l'esperienza del cambiamento politico. In epoca comunista, entrambi i paesi vivevano in condizioni di piena occupazione ${ }^{10}$. In Ungheria il picco numerico della classe operaia fu raggiunto nel 1970, quando le industrie di stato impiegavano 1,4 milioni di lavoratori industriali, il $58 \%$ di tutta la manodopera attiva ${ }^{11}$. La situazione in Germania est era simile: è importante ricordare che negli anni ' 80 un operaio specializzato tedesco dell'est guadagnava più di un giovane ingegnere o ricercatore e decisamente più di un segretario ${ }^{12}$.

La Wende (il cambio di regime) ebbe un impatto diverso in Ungheria e nella Ddr. Nel 2004 e nel 2008, per quanto il salario medio della Germania est fosse ancora più basso di quello della Germania ovest (circa il 70-72\%), il Pil pro capite tedesco era superiore di circa il $16 \%$ alla media europea, mentre quello ungherese era pari al 63\% della media Ue nel 2004 ed al 64\% nel $2008^{13}$. Se definiamo poveri coloro che vivono a un livello di consumi pari alla metà della media Ue, allora quasi i 3/4 degli ungheresi possono essere considerati tali ${ }^{14}$. Per quanto il tasso di disoccupazione in Germania est fosse all'epoca delle interviste più alto di quello ungherese (al 20\%, mentre in Ungheria esso ha superato il $10 \%$ solo nel 2009), in Ungheria il tasso di occupazione è molto basso: mentre nel 2009 in Germania il 71\% della popolazione fra i 15 ed i 64 anni era occupata, in Ungheria la percentuale era solo del $55 \%$. Ferge stima che in Ungheria in seguito alle ristrutturazioni economiche si sia perso un milione di posti di lavoro mentre, e secondo i calcoli di Mark Pittaway nel 2008 vi era il $23 \%$ di posti di lavoro in meno che nel $1989^{15}$.

\footnotetext{
${ }^{10}$ Secondo studi ungheresi, i rom ungheresi rimanevano svantaggiati. Nel 1970 solo il $30 \%$ delle romnja (donne rom) in età lavorativa erano ufficialmente occupate, e ancora nel 1987 la percentuale non superava il 49\% (ricadendo bruscamente nel 1993 al 16\%). Cfr. B. Janky, A cigány nók társadalmi helyzete és tevékenysége, in I. Nagy, T. Pongrácz e I. Gy. Tóth, Szerepváltozások. Jelentés a nók és férfiak helyzetéról. Tárki, Budapest 2005, pp. 136-48, in part. p. 139. Cfr. anche S. Zimmermann, Gender Regime and Gender Struggle in Hungarian State Socialism, «Aspasia», 4 (2010), pp. 1-24. Nel 1994 Kemény stimava che la popolazione rom in Ungheria comprendesse mezzo milione di individui (il 5\% della popolazione): cfr. I. Kemény (a cura di), Beszámoló a magyarországi roma (cigány) népesség helyzetével foglalkozó, 1993. október és 1994. február között végzett kutatásról, Mta Szociológiai Intézete, Budapest 1995, pp. 4-5.

${ }_{11}$ Cfr. T. Valluch, Magyarország társadalomtörténete a XX. század második felében, Osiris, Budapest 2001.

${ }_{12}$ Cfr. H. Stephan-E. Wiedemann, Lohnstruktur und Lohndifferenzierung in der Ddr. Ergebnisse der Lohndatenerfassung vom September 1988, «Mittelungen aus der Arbeitsmarkt und Berufsforschung», 23 (1990), pp. 550-62.

$13 \mathrm{http}: / /$ epp.eurostat.ec.europa.eu/tgm/table.do?tab=table\&init=1\&plugin=1\&language=en $\&$ pcode $=$ tsieb010, consultato il 15 ottobre 2010.

${ }_{14}$ Cfr. Zs. Ferge, Társadalmi áramlatok és egyéni szerepek, Napvilág Kiadó, Budapest 2010.

${ }_{15}$ Cfr. M. Pittaway, A magyar munkásság és a rendszerváltás', «Múltunk», n.s., 56 (2011), n. 1, pp. 4-18.
} 
Secondo le stime di Ferge (2010), il 45-50\% degli ungheresi ha perso qualcosa nel cambio di regime, il 30-35\% non ha visto alcun cambiamento e il $25 \%$ ne ha tratto profitto. Va sottolineato che la povertà sembra essere in Ungheria una condizione "duratura": secondo un sondaggio, su 3.000 intervistati la maggioranza (60\%) di coloro i quali erano poveri nel 1992 si trovavano nella stessa situazione 15 anni più tardi, e solo il $7 \%$ era riuscito a migliorare le proprie condizioni ${ }^{16}$. Tali dati suggeriscono che la società ungherese stia diventando più chiusa.

È necessario mettere in luce anche le diversità politiche: gli ungheresi già nel 2000 riferivano di aver sperimentato una notevole insicurezza riguardo ai redditi e alla sicurezza sociale, ed era generalmente provato che i poveri privilegiassero la sicurezza rispetto ai valori della democrazia e della libertà personale ${ }^{17}$. In Ungheria, l'estrema sinistra non compare più nell'agone politico, mentre in Germania vi è un forte partito di sinistra radicale, la Linke. Infine, i sentimenti ostili ai poveri ed ai rom e genericamente razzisti sono andati aumentando in Ungheria fin dalla crisi del 2008, fenomeno al quale non è estranea la grande presenza mediatica dell'estrema destra su canali televisivi e sui giornali; la formazione di destra radicale Jobbik è diventata il terzo partito alle elezioni politiche del $2010^{18}$.

Tali differenze risultano anche dalle storie di vita degli intervistati. Sulla base di queste ultime, ho distinto tre dimensioni di analisi dell'esperienza del post-socialismo: il mondo del lavoro; la valutazione soggettiva degli standard di vita e del livello di integrazione nella società dei consumi; le relazioni interpersonali. La prima dimensione presenta due differenti tipologie di esperienze: in entrambi i gruppi, metà degli intervistati ha avuto la possibilità di vivere la transizione all'interno della fabbrica, mentre l'altra metà ha perso il lavoro o è stata mandata in pensione anticipata. La transizione al post-fordismo è stata esperita in maniera essenzialmente diversa nei due paesi ${ }^{19}$. I lavoratori della Rába hanno costruito all'unanimità una «narrazione del declino» attorno alla vicenda post-socialista della loro fabbrica: i manager hanno diminuito la produzione, i nuovi proprietari hanno rifiutato di investire nell'innovazione e nello sviluppo tecnico dello stabilimento, hanno tratto i loro profitti dalla liquidazione del ricco patrimonio della Rába e hanno licenziato gli operai che lavoravano lì fin da quando gli impianti erano stati costruiti dal leggendario manager comunista Ede Horváth. Secondo molti, la nuova proprietà

${ }^{16}$ Cfr. Zs. Ferge, Társadalmi cit., pp. 165-67. 34.

17 Id., Vágányok és vakvágányok a társadalompolitikában, L’Harmattan, Budapest 2012, p.

${ }^{18}$ Sulle tendenze anti-rom cfr. J. Ladányi, Leselejtezettek: A kirekesztett népesség társadalom-és térszerkezeti elhelyezkedésének átalakulása Magyarországon a piacgazdasági átmenet időszakában, L'Harmattan, Budapest 2012. Jobbik è l'abbreviazione di Jobbik Magyarországért Mozgalom, Movimento per un'Ungheria migliore.

19 Per un'autorevole critica di sinistra al post-fordismo cfr. L. Boltanski-E. Chiapello, The New Spirit of Capitalism, Verso Books, London 2005. 
avrebbe fatto crollare intenzionalmente la produzione per poter guadagnare dalla vendita dei beni aziendali. Le rimostranze della manodopera sono così spesso sfociate, come vedremo, in articolate teorie del complotto.

Szalai opera una distinzione fra i lavoratori del settore multinazionale e quelli del settore domestico, descrivendo questi ultimi come dei bricoleurs sfruttati e sottopagati, sovente assunti in nero e costretti a vivere alla giornata ${ }^{20}$. I lavoratori della Rába sembrano aver fatto proprio un modello simile, identificandosi totalmente nella figura del bricoleur e associando lo sviluppo (post)industriale con le imprese multinazionali che hanno distrutto le antiche eccellenze dell'industria domestica, attratto i loro lavoratori migliori e imposto una concorrenza sleale alle impoverite aziende nazionali.

Perché si sa che in Occidente lo stato protegge le imprese nazionali. Ma guarda la fabbrica di vagoni ${ }^{21}$. Era un'impresa redditizia e credo che ora ci sia una volontà di distruggerla per eliminare la concorrenza. Io non mi faccio ingannare. Győ́r aveva delle famose fabbriche tessili, sono state tutte vendute ai concorrenti stranieri [occidentali], che le hanno tutte chiuse o fatte a pezzi in qualche altro modo?

Il mio figlio più giovane lavora alla Audi [alla Rába]. Lì gli ungheresi li sfruttano solo. Li fanno lavorare più duro di noi, ma se faccio il paragone fra quanto sono pagati laggiù [a Ingolstad, in Germania] e quanto è pagato lui, non c'è dubbio che siano sfruttati. Non vengono qui per farci un favore ma per guadagnare, perché la nostra forza lavoro è più economica. Non avrebbero dovuto permettere che gli ungheresi venissero sfruttati in questo modo. I politici si accusano a vicenda invece di impedire la svendita di tutto il paese ${ }^{23}$.

Le citazioni mostrano chiaramente come le rimostranze dei lavoratori si siano trasformate in un discorso etnico-populista, in cui il capitale "multinazionale" (occidentale) viene identificato con l'élite domestica "traditrice"; entrambi sono accusati di distruggere l'industria ungherese e di essere quindi responsabili della miseria dei lavoratori, che hanno perso l'esistenza sicura, garantita loro dal regime di Kádár. Per sottolineare l'attuale declino, molti lavoratori contrappongono esplicitamente ai «duri anni '90» l'età gloriosa della Rába sotto Ede Horváth, quando i suoi prodotti venivano esportati in tutti i paesi del Comecon e negli Stati Uniti e la fabbrica veniva presentata dai media e dalla stampa come una fabbrica socialista modello.

Ai vecchi tempi era un onore lavorare alla fabbrica di vagoni. Ero così orgogliosa quando mio padre mi portò lì la prima volta a diciott'anni, pensando che avrei lavorato nella famosa fabbrica di vagoni... e adesso eccomi qui [sospiro]. Se mi licenzia-

20 Cfr. E. Szalai, Tulajdonviszonyok, társadalomszerkezet és munkásság', «Kritika», 33 (2004), n. 9, pp. 2-6

${ }^{21}$ Denominazione locale della Rába.

${ }^{22}$ Intervista con Péter (49 anni), ungherese, operaio specializzato e rappresentante sindacale alla Rába, 2002.

${ }^{23}$ Intervista con Brigitta (51 anni), operaia specializzata alla Rába, 2002. 
no, non so cosa farò. Distribuirò giornali, farò le pulizie negli uffici o nelle case... purtroppo, non c'è nient'altro. E fa paura! E poi mi sono sposata tardi, mia figlia ha appena cominciato le superiori e mio figlio andrà all'università il prossimo anno. Se fossimo solo io e mio marito non andrebbe così male. Ma devo sostenerli, sono tutt'e due studenti eccellenti. E questo è il mio problema principale, perché devono andare entrambi all'università, perché non posso permettere che dopo le superiori vadano a lavorare ${ }^{24}$.

I lavoratori ungheresi erano unanimemente d'accordo nel considerare la storia della loro fabbrica dopo il 1989 una storia di declino, di cui incolpavano a livello locale la dirigenza e a livello nazionale le multinazionali e lo Stato, che non era riuscito a difendere le imprese efficienti. Le innovazioni e gli sviluppi post-fordisti erano rappresentati dalla Audi, che essi consideravano la fonte di umiliazione della loro impresa: l'Audi aveva rilevato il gigantesco impianto costruito da Ede Horváth per riportare la produzione di automobili a Győr. I lavoratori della Rába ricordavano amaramente come sotto Horváth la loro azienda fosse la principale benefattrice della città: aveva costruito uno stadio e poteva vantare una squadra di calcio, una casa della cultura, una biblioteca ben fornita, un'orchestra, un coro e una compagnia di danza. Dopo il cambio di regime il ruolo di principale benefattrice di Gyốr passò all'Audi, secondo i lavoratori della Rába a causa delle inique esenzioni fiscali (delle quali venne incolpato il governo).

L'esperienza della Zeiss è ben diversa dalla «narrazione del declino» diffusa fra i lavoratori della Rába. L'azienda mise in atto un massiccio piano di licenziamenti: il presidente del consiglio d'impresa (Betriebsrat) stimava che circa 16.000 persone avessero perso il loro lavoro nei primi anni dopo la Wende. A essere licenziati furono soprattutto i più giovani, i quali avrebbero potuto trovare più facilmente dei padri di famiglia di mezza età, si pensava, lavoro all'Ovest. Nel 1995 furono licenziati altri 600 operai $^{25}$. Il quadro della Zeiss appare però più sfumato della situazione ungherese. I lavoratori vissero in maniera positiva il passaggio al modello post-fordista: il nuovo proprietario, la Zeiss della Germania ovest, modernizzò gli impianti, adottò nuovi macchinari e tecnologie e fece significativi investimenti a Jena. Gli operai conobbero un miglioramento delle condizioni di lavoro (salari competitivi, installazioni di impianti di condizionamento, nuovi bagni e nuove mense, orari di lavoro flessibili). Tuttavia, essi lamentavano di lavorare in condizioni di stress e tensione maggiori rispetto al vecchio modo di produzione.

\footnotetext{
${ }^{24}$ Intervista del 2002 con Judit (50 anni), operaia specializzata ungherese alla Rába, con diploma di scuola superiore.

${ }_{25}$ Notizie desunte dall'intervista del 2003 con Thorsten (52 anni), presidente del consiglio d'impresa della Zeiss, operaio prima del 1989 e membro dell'opposizione ecclesiastica. Aveva ricevuto un'educazione religiosa, subendo per questo discriminazioni a scuola, e gli era stata negata l'ammissione ad una scuola d'arte. Una sua sorella era emigrata in Germania ovest, il che lo aveva reso ancora più sospetto agli occhi delle autorità. Dopo la Wende partecipò alla riorganizzazione dei sindacati.
} 
Le «narrazioni del declino» sono sostanzialmente assenti nelle interviste della Germania est. A differenza degli ungheresi, gli operai, incluso un ex-segretario di partito che dichiarava di essere ancora comunista, non facevano cenno a casi di corruzione e di truffa in seguito alla privatizzazione. Piuttosto, essi spiegavano i massicci licenziamenti con il collasso del Comecon e l'aumento dei costi di produzione.

La disoccupazione fu senza dubbio l'esperienza peggiore che gli intervistati della Germania est dovettero affrontare in seguito al cambio di regime. A Gyốr, al contrario, questo era un timore molto meno presente ${ }^{26}$. Gli intervistati ungheresi erano convinti che chiunque avesse voglia di lavorare potesse trovare "qualcosa" a Győr; in effetti, i sentimenti anti-rom venivano spesso giustificati con la teoria che i nomadi, che vivevano grazie alla sicurezza sociale e agli assegni familiari, avrebbero potuto trovare lavoro se solo l'avessero voluto veramente. Per i lavoratori della Germania est la privatizzazione non fu associata alla corruzione, alla decadenza dell'azienda e all'affermazione di un concorrente occidentale quale fu l'Audi a Gyór. Tuttavia, la disoccupazione fu una costante fonte di tensioni e di paure, che tutti gli intervistati dovettero fronteggiare o direttamente o attraverso le vicende di figli, parenti, compagni. Una disoccupazione di lungo periodo significava non soltanto l'esclusione da un mondo del lavoro rispettato, ma anche un isolamento sociale che spesso aveva pesanti conseguenze psicologiche. Alcune intervistate raccontarono addirittura delle lunghe terapie e del finale suicidio dei loro mariti, da tempo disoccupati:

Dopo la Wende, mio marito divenne libero professionista. Ci trasferimmo ad Amburgo, ma gli affari andarono male e dovette dichiarare bancarotta. Egli non riuscì ad adattarsi al cambio di regime, divenne completamente passivo, depresso. Cercai di aiutarlo in tutti i modi, ma non ci riuscii. Non mi sento in colpa. Volevo tornare a Jena, tutti i miei amici erano lì. Lui rimase ad Amburgo, ci vedemmo un paio di volte e mi disse che sarebbe tornato a Jena ma morì un anno e mezzo fa. No, non era depresso [più avanti: si suicidò] Non era colpa sua... non riusciva a trovare il suo posto dopo la bancarotta 27 .

L'intervista dimostra come il peggiore aspetto della disoccupazione non fosse il declino materiale (per quanto anch'esso ricordato), bensì la perdita

${ }^{26} \mathrm{Al}$ momento dell'intervista, il tasso ufficiale di disoccupazione a Győr era inferiore al $5 \%$, mentre a Jena era il doppio.

27 Intervista con Dora (56 anni), nel 2004 madre disoccupata. Aveva iniziato la carriera come operaia specializzata alla Zeiss; ottenuto un posto alla Facoltà di economia e ingegneria, terminò gli studi pur lavorando grazie alla Zeiss, che sosteneva la formazione degli adulti, concedendo agli operai giorni di permesso per studio. Anche il marito di Dora era entrato nel programma di formazione degli adulti, ed entrambi erano divenuti ingegneri dell'azienda. Nella Germania ovest, tuttavia, i diplomi delle "scuole marxiste" non costituivano sempre titoli di merito, e coloro che avevano seguito queste scuole erano spesso considerati "comunisti indottrinati”. 
del rispetto degli altri, che aveva conseguenze psicologiche estremamente gravi. In molti casi gli intervistati che erano da lungo tempo senza lavoro dichiararono che i loro amici, colleghi, conoscenti rifiutavano di credere che non riuscissero a trovare un impiego, e in alcuni casi li avevano addirittura accusati di essere dei fannulloni che vivevano grazie alla previdenza sociale. Molti avevano deciso di isolarsi per evitare simili rimproveri. Tutti coloro che accettarono di farsi intervistare affermavano di essersi coscientemente sforzati di non cadere in tale trappola: avevano fatto ricorso alle reti di relazioni che si erano formate nella Ddr, erano entrati in nuove associazioni (per esempio, un'operaia lavorava come volontaria per il sindacato) o in gruppi di sostegno (il figlio di una delle intervistate, che non riusciva a trovare un lavoro fisso da molti anni, era entrato in un gruppo di disoccupati che si spartivano lavori occasionali).

Anche nell'ambito della seconda dimensione (la valutazione soggettiva degli standard di vita), possiamo osservare significative differenze fra i due gruppi. La stragrande maggioranza degli intervistati tedeschi riferì di miglioramenti nelle proprie condizioni materiali: coloro che avevano un lavoro descrivevano una condizione di prosperità materiale che aveva loro permesso di costruire villette familiari, comprare nuove macchine e passare le vacanze in esotiche località estere, mentre i disoccupati parlavano positivamente del miglioramento dei servizi e della disponibilità di beni di consumo. Gli intervistati ungheresi, per contro, presentavano la loro condizione materiale come la prosecuzione delle «narrazioni del declino»: tutti parlavano del ristagno o della decadenza dei propri standard di vita, considerata la più dura fra le esperienze legate al cambio di regime. Il regime di Kádár era prevedibile; per quanto la manodopera qualificata urbana ammettesse che questo non forniva grandi prospettive, alcuni obbiettivi apparivano realisticamente perseguibili: un appartamento in città o una casa indipendente in campagna, una macchina, una gita nel week-end e vacanze regolari. Il nuovo sistema non offriva simili prospettive; anche coloro i quali affermavano di aver conservato il proprio livello di vita precedente spiegavano che ciò era dovuto al fatto di non avere più figli a carico, eventualità che li avrebbe costretti a ripiegare su una qualità di vita inferiore. Coloro che avevano figli in età scolare parlavano amaramente dell'aumento delle nuove diseguaglianze materiali:

I miei figli non sono esigenti e capiscono bene che non possiamo permetterci quello che si permettono altri. Io però mi sento in colpa, perché sono costretti a rinunciare a così tante cose...Quando c'è una gita scolastica e andiamo a prendere nostro figlio, dico sempre a mio marito: lascia la macchina sul retro del parcheggio così gli altri bambini non vedono che macchina vecchia che abbiamo ${ }^{28}$.

Dalle interviste ungheresi traspare come la perdita di sicurezza materiale sia stata un'esperienza dominante nella Wende: la stragrande maggioranza dei

${ }^{28}$ Intervista con Judit (50 anni), operaia ungherese alla Rába, 2002. 
lavoratori dichiarava di vivere peggio rispetto al passato. Per far quadrare il bilancio familiare, molti intervistati avevano dovuto rinunciare a "lussi" quali automobile, viaggi, cene al ristorante (eccezion fatta per quelli economici). Coloro che vivevano in famiglie monoreddito si erano trovati in una situazione finanziaria particolarmente difficile, e affermavano di essere andati incontro a un drastico declino. Ho intervistato un'operaia specializzata che, divorziata, durante il regime di Kádár aveva mantenuto i suoi tre figli con un solo stipendio finché non aveva incontrato il secondo marito. All'epoca dell'intervista, viveva con una pensione di invalidità. Il nuovo marito era un tecnico alla Rába ed avevano avuto un figlio insieme. Dopo la malattia di lei, la famiglia aveva venduto l'appartamento in città e si era trasferita in un villaggio vicino, nella speranza che la vita fosse meno cara:

A Győr vivevamo in un condomino, il riscaldamento era molto caro e abbiamo pensato che sarebbe stato più economico stare in campagna. Abbiamo speso tutti $\mathrm{i}$ nostri risparmi, e adesso letteralmente i soldi ci bastano appena per arrivare a fine mese. Manteniamo un solo figlio e spendiamo solo per le necessità di base, perché gli stipendi sono così bassi. Mio marito guadagna 100.000 fiorini $^{29}$, ma togliendo le tasse ce ne restano 70.000, compreso l'assegno familiare. Ed è un tecnico specializzato. Negli anni '80 vivevamo molto meglio, e all'epoca avevamo quattro figli da mantenere. Li nutrivamo, li mandavamo a scuola e potevamo ancora permetterci un'auto, una Tv, un videoregistratore e altre cose. Ora non possiamo comprarci niente. Penso che il sistema di Kádár fosse molto meglio per noi che quello attuale. Perché dava qualcosa anche ai poveri. Non c'erano grandi differenze fra le persone. Oggi, in Ungheria ci sono un milione e mezzo di veri poveri ${ }^{30}$.

Mentre gli intervistati ungheresi ritenevano all'unanimità che la classe lavoratrice fosse quella che più aveva perso nel cambio di regime, i tedeschi orientali criticavano piuttosto la cristallizzazione delle gerarchie sociali nel nuovo sistema. I disoccupati si sentivano consumatori di "seconda classe" nella società tedesca perché potevano permettersi molto meno dei loro conoscenti con un lavoro. Tuttavia, mentre in Ungheria molti lavoratori continuavano a giudicare negativamente il governo a causa degli scarsi risultati ottenuti nel migliorare il loro tenore di vita, i tedeschi non esprimevano alcun desiderio di tornare all'epoca di Honecker. Nemmeno Jan, che nel nuovo regime si era trovato senza casa e senza lavoro, riteneva lo «stato dei lavoratori» un'alternativa plausibile. Nel caso della Germania est, possiamo osservare un graduale slittamento verso valori post-materialistici: la disoccupata Dora avrebbe potuto trovare un lavoro ad Amburgo ma aveva deciso di vivere a Jena per stare vicina ai suoi amici; numerosi lavoratori richiamavano l'attenzione sulle nuove tecnologie a basso impatto ambientale che avevano reso più salubre l'aria delle città; molti criticavano esplicitamente il consumismo

\footnotetext{
29 Valuta ungherese, attualmente del valore di circa 0,0036 euri.

30 Intervista con Éva (54 anni), operaia specializzata ungherese, 2004 (corsivo mio).
} 
fine a se stesso ed alcuni partecipavano a gruppi di sostegno reciproco o svolgevano una qualche forma di volontariato. In Ungheria, i lavoratori si lamentavano esplicitamente della perdita delle reti di relazione esistenti; nessuno parlava di volontariato; e molte lavoratrici rurali dichiaravano espressamente di desiderare il ritorno del regime di Kádár, quando la vita delle loro famiglie era migliore e più sicura. Nel caso ungherese, i valori materiali continuavano a dominare il pensiero politico. Non vedendo valide alternative al consumismo, gli intervistati esprimevano prevalentemente un senso di perdita e di frustrazione.

La percezione della mancanza di integrazione sociale era direttamente connessa alle relazioni interpersonali. In questo ambito va innanzitutto rilevata la presenza di una generica critica alla società capitalista, che si può spiegare con la comune provenienza da un sistema che propugnava un maggiore egualitarismo. In entrambi i gruppi, gli intervistati raccontavano di un peggioramento delle relazioni interpersonali: le comunità operaie erano state distrutte in seguito ai licenziamenti e all'aspra competizione per il lavoro; le persone si trovavano isolate e atomizzate sul posto di lavoro; la solidarietà andava scomparendo e ognuno era concentrato unicamente su se stesso; gli individui riducevano deliberatamente i contatti personali per paura di aprirsi e di mostrare le proprie debolezze, temendo che queste potessero essere utilizzate contro di loro. Gli intervistati tedeschi utilizzavano termini militari per descrivere l'intensificazione della competizione: parlavano di combattenti solitari (Einzelkämpfer), società dei due terzi (Zwei-Drittel Gesellschaft ${ }^{31}$ e società ipercompetitiva (Ellbogengesellschaft, letteralmente «società in cui ci si fa largo a gomitate»). In entrambi i gruppi, gli intervistati ricordavano la collegialità e l'intensa vita comunitaria all'epoca del socialismo con un senso di perdita:

Una volta c'erano periodicamente momenti di festa nei condomini, in cui i vicini stavano insieme e parlavano. Oggi nessuno vuole qualcosa di simile. Non vogliono stare insieme e parlare delle loro cose perché potrebbero rivelare qualcosa che vada a vantaggio degli altri. Oggi ognuno è spaventato di condividere le proprie opinioni, le proprie cose o i propri problemi con gli altri perché potrebbe metterlo in posizione di svantaggio. Secondo me oggi il regime non vuole delle vere comunità, sul posto di lavoro e da nessuna altra parte. Al di sotto di un certo livello non vogliono nemmeno veri contatti intimi fra le persone ${ }^{32}$.

${ }^{31}$ Una società in cui $2 / 3$ della popolazione appartiengono alle classi medie o alte. In Germania si è calcolato che questi fossero gli occupati, mentre il restante terzo era condannato a vivere del sussidio di disoccupazione, degli assegni sociali e/o del lavoro nero. Gli intervistati ungheresi non utilizzavano questo termine; tuttavia, la citazione precedente induce a pensare che essi avrebbero piuttosto fatto propria un'accezione opposta di società dei due terzi, sviluppatasi nell'Europa postsocialista, in base alla quale 2/3 della società avevano perso lo status di classe media.

32 Intevista con Karl (51 anni), corsivo mio. 
Mentre gli ungheresi generalmente imputavano il deterioramento della loro situazione materiale alla diminuzione delle loro relazioni sociali (non potevano infatti più permettersi ristoranti, feste, vacanze in gruppo) ${ }^{33}$, per i tedeschi dell'est la disintegrazione delle vecchie comunità era frutto della dura competizione, tipica del nuovo sistema. A loro parere, lo sviluppo tecnologico rendeva superflua una parte della società e induceva quindi una competizione per il lavoro più aspra di quella sperimentata in passato. Ne conseguivano un'intensa individualizzazione della società, la perdita del vecchio spirito collettivo solidaristico, una più dura lotta con i concorrenti sul posto di lavoro, la riduzione dei legami privati fra colleghi, l'esigenza della segretezza (per evitare che altri approfittassero delle proprie informazioni) e la generale atomizzazione. I lavoratori di entrambi i gruppi ribadivano che durante il regime socialista le persone si relazionavano fra di loro in maniera differente: le comunità erano più forti e i rapporti interpersonali meno orientati verso il profitto, la promozione sociale e gli interessi materiali, mentre erano molti di più coloro che erano disposti a lavorare gratis come volontari per la comunità. La disintegrazione delle comunità legate al posto di lavoro fu insomma un'esperienza negativa per entrambi i gruppi; non è un caso, quindi, che questo fosse il punto su cui le due critiche al nuovo regime si avvicinavano maggiormente.

Gli ungheresi parlavano soprattutto di declino della classe media (alla quale sentivano di appartenere), laddove i tedeschi orientali criticavano il crescente divario fra le diverse componenti di essa (professori, medici, avvocati e manager) e gli operai. Molti ricordavano il clima più egualitario della Ddr con un esplicito senso di perdita:

Oggi la gerarchia [lavorativa] è molto più evidente che nella Ddr, questo è chiaro. Una volta, il regime promuoveva l'egualitarismo, le persone dovevano restare unite, dovevano sempre essere parte di una comunità e una tuta blu doveva essere rispettata tanto quanto un ingegnere. Oggi si dice esattamente l'opposto: che devono esserci differenze sempre maggiori fra le persone, che la condivisione è sbagliata e la sola cosa che conta è quanto hai - il mio cavallo, la mia casa, la mia riserva di vini e così via. Cosa pensate che succederà allora? È ovvio, le differenze aumenteranno ${ }^{34}$ !

Bisogna comunque rilevare che per i lavoratori della Germania est non era negativo che i loro figli diventassero operai: si preoccupavano soltanto che scegliessero una professione o un settore che garantisse loro lavoro. Gli ungheresi, al contrario, consideravano la condizione operaia senza speranze: tutti coloro che volevano assicurare il futuro dei loro figli pensavano di man-

\footnotetext{
33 Secondo l'inchiesta condotta da Utasi su tutta l'Ungheria, le classi inferiori potevano contare solo sui propri nuclei familiari e il livello di fiducia sociale nel paese era molto basso: cfr. Á. Utasi, Éltető kapcsolatok: A kapcsolatok hatása a szubjektív életminôségre, Új Mandátum, Budapest 2008.

34 Intervista con Jörg (57 anni), operaio specializzato tedesco alla Zeiss, 2003.
} 
darli all'università e tutti convenivano che in Ungheria un operaio non potesse condurre una vita normale. Amaramente, ricordavano che all'epoca di Kádár potevano risparmiare abbastanza da comprarsi una casa propria, mentre nel nuovo regime non vedevano alcuna possibilità che i loro figli potessero farlo senza l'aiuto dei genitori o dei nonni.

L'analisi comparativa fin qui svolta mette in luce come le differenze strutturali fra i due paesi abbiano dato luogo a maniere essenzialmente diverse di vivere la svolta post-socialista. In Ungheria, la percezione della propria condizione periferica nel sistema post-fordista sfociò nell'elaborazione da parte degli operai di «narrazioni del declino» che imputavano il mancato aggancio al treno dello sviluppo a fattori esterni e sovente facevano ricorso a teorie del complotto. D'altra parte, la comune critica alle forme assunte dai rapporti interpersonali nel nuovo regime suggerisce che in epoca socialista i lavoratori avessero condiviso la medesima esperienza umana, ricordata con nostalgia e in termini piuttosto simili da entrambi i gruppi. Le paure per il presente erano però diverse: gli ungheresi temevano principalmente il declino delle condizioni materiali, i tedeschi dell'est erano maggiormente spaventati dalla disoccupazione. Soprattutto, il discredito che tale vissuto comune gettava sul nuovo regime era molto minore fra i tedeschi orientali che fra gli ungheresi. Gli intervistati ungheresi non avevano avuto esperienza diretta del cambiamento politico; nessuno di essi aveva preso parte alle manifestazioni e molti prendevano le distanze dal 1989:

Per me non aveva importanza avere voce in capitolo in politica. Non voglio abbellire la realtà, ma per me [le elezioni libere] non erano così importanti. Se devo essere onesto, la mia esistenza era sicura, vivevo la mia vita e allevavo i miei figli. Ero arrivato a tutto quello che potevo avere al mio livello. Per me, su quali cose dovessi avere voce in capitolo non era poi così importante. Sinceramente, questa è la mia rispo$\mathrm{sta}^{35}$.

Mentre i tedeschi orientali si identificarono con la Wende (perché non amavano la dittatura di Honecker, perché erano favorevoli alla riunificazione delle due Germanie o per entrambi i motivi), gli ungheresi sentirono che quello non era il loro cambio di regime. Per la maggioranza, si trattava di "affari" dell'élite: e man mano che la situazione materiale andò peggiorando persero fiducia nelle istituzioni democratiche, ritenute origine della corruzione, del dominio dei ricchi sui poveri e delle pratiche disoneste e truffaldine che tutti associavano alla privatizzazione:

Non so chi ci guadagnò dal 1989. La mia vita era più tranquilla sotto il socialismo, e penso che la maggioranza degli ungheresi vivesse meglio con il regime di Kádár [rispetto ad oggi]. Quando è venuta questa democrazia, hanno venduto quello che si poteva vendere in questo paese. Credo che privatizzare gli ospedali, le indu-

\footnotetext{
35 Intervista con Péter (49 anni), operaio ungherese alla Rába, 2002 (corsivo mio).
} 
strie elettriche e del gas, le ambulanze sia una cosa orribile, perché i nuovi proprietari deruberanno i lavoratori di tutti i loro risparmi e le loro proprietà. Questo ci insegnavano alla scuola di partito, ed è vero. Oggi l'Ungheria è governata da un capitalismo di rapina. Non ci sono regole, leggi e rispetto della morale. Ciascuno ruba tutto quello che può ${ }^{36}$.

I simpatizzanti della sinistra erano fortemente contrari alla privatizzazione, ma anche coloro che si dichiaravano anti-comunisti "militanti" avevano un'opinione fortemente negativa di essa e del funzionamento del capitalismo in generale - la sola differenza era che questi ultimi incolpavano del malfunzionamento del capitalismo ungherese i funzionari comunisti, che ritenevano continuassero a governare il paese:

Da giovane, il mio sogno era di diventare lavoratore in proprio: in termini moderni, imprenditore. Ma odio questo nuovo termine, perché ormai viene usato praticamente per qualunque cosa. Nessuno tiene più in considerazione le reali capacità individuali. Se ho i soldi, posso aprire un ristorante, una salone di bellezza o una farmacia, ma non vuol dire che sappia qualcosa di quel settore o di quella professione. Se hai i soldi, non hai bisogno di sapere niente; semplicemente, assumi persone pratiche del settore. Ma non paragonerei mai questo con gli imprenditori del passato, che conoscevano il loro mestiere. Penso che l'imprenditoria sia grandemente decaduta. Quelli che lavorano duro hanno perso importanza nel sistema. La sola cosa che conta è come vendi le cose - nessuno è interessato alla qualità. A mio parere, è un sistema molto superficiale e con valori molto superficiali ${ }^{37}$.

Secondo David Ost, in Polonia gli intellettuali liberali avevano tradito l'alleanza con la classe lavoratrice che si era formata all'epoca di Solidarność, e per tutta risposta gli operai delusi avrebbero deciso di votare per la destra o l'estrema destra, che prometteva di ristabilire l'orgoglio nazionale e di difendere gli interessi dell" "uomo della strada" 38 . Nel caso ungherese non si può parlare di alleanza fra lavoratori e intellettuali dopo il 1956; la mia ricerca mostra come i lavoratori non avessero familiarità con i concetti di autogoverno e di autogestione elaborati dagli intellettuali di sinistra critici verso il so-

\footnotetext{
${ }^{36}$ Intervista del 2004 con Tibor (67 anni), dirigente in pensione, che aveva iniziato la carriera come operaio specializzato alla Rába, ottenendo un diploma grazie al programma di formazione degli adulti.

37 Intervista del 2004 con Miklós (51anni), lavoratore in proprio (idraulico), che aveva cominciato come operaio specializzato alla Rába; era stato due anni in Urss come lavoratore ospite, un «buon affare» perché gli stipendi erano molto alti. Come affermava orgogliosamente, di questo doveva ringraziare solamente il suo lavoro perché non era mai stato iscritto al partito e non amava i comunisti (suo padre era un contadino le cui terre erano state nazionalizzate e non aveva mai perdonato i comunisti). Divenuto lavoratore autonomo nel 1981, Miklos negli anni ' 90 aveva allargato il suo giro d'affari senza però riuscire a sostenere lo stress; dopo un'operazione, aveva lasciato la professione accettando un lavoro come addetto alla manutenzione. Lavorava anche in nero per garantirsi delle entrate "normali".

${ }^{38}$ D. Ost, The Defeat of Solidarity: Anger and Politics in Postcommunist Europe, Cornell UP, Ithaca 2005.
} 
cialismo di stato, e molti intervistati non consideravano le elezioni libere come qualcosa di davvero importante per la loro vita o la loro identità ${ }^{39}$. La corruzione, di cui avevano avuto esperienza diretta con la privatizzazione, aveva fortemente indebolito ai loro occhi la credibilità delle istituzioni democratiche e dell'economia di mercato; invece della prosperità promessa (ed attesa), queste avevano offerto loro solo la stagnazione o il netto declino del tenore di vita, nonché l'esperienza di una crescente disuguaglianza fra gli operai e le nuove classi borghesi (manager, banchieri, avvocati, medici e uomini d'affari - in altre parole, coloro che possono essere considerati i vincitori nel passaggio di regime). Come i loro omologhi polacchi, numerosi operai ungheresi erano sensibili alle parole d'ordine nazionaliste e populiste, che proponevano un'immagine concreta del nemico: "stranieri", capitalisti sfruttatori, aziende multinazionali che portavano i profitti all'estero, ecc. La loro sensazione di ressentiment era inoltre accresciuta dai "consumi vistosi" della nuova élite, che rendevano molto più evidente il loro impoverimento. L'idea che ciò fosse conseguenza dell'indebolimento dello stato trovò un'ampia audience: gli operai invocavano un governo forte, che prendesse una posizione decisa contro il capitale globale.

Gli operai ungheresi nutrivano insomma numerosi dubbi sulla Wende e sulla nuova democrazia, che molti non sentivano come propria. Tuttavia, essi non riuscivano a trasformarli in una critica complessiva del capitalismo, auspicando piuttosto un particolare modello di capitalismo ungherese, in cui il governo si facesse mediatore fra gli interessi dei lavoratori e quelli dei capitalisti. Le ragioni per le quali la sinistra ungherese non riuscì ad approfittare della disillusione dei lavoratori verso il "capitalismo esistente" sono varie. Lasciando da parte le ricordate differenze di mentalità fra operai tedeschi e ungheresi, vale la pena sottolineare l'assenza in Ungheria di un'opinione pubblica anticapitalista di sinistra; anche gli elettori di sinistra impegnati ritenevano che nessuno dei partiti esistenti rappresentasse gli interessi del lavoro. La plateale esclusione della classe operaia dall'agone politico ungherese e la debolezza del movimento sindacale rafforzò così la fiducia in uno stato e in un governo forti: i lavoratori pensavano che lo stato fosse al di sopra delle classi, e di conseguenza avrebbe fatto qualcosa per l' "uomo della strada".

Non sarebbe corretto affermare che i tedeschi dell'est non fossero critici verso la nuova democrazia. Tuttavia essi, a differenza degli ungheresi, non

\footnotetext{
39 Queste considerazioni sono in linea con lo studio di Haraszti sulla fabbrica di trattori Stella rossa (Vörös Csillag Traktorgyár). Gli operai avevano esplicitamente detto all'autore che non sarebbe rimasto a lungo nella fabbrica perché aveva un'istruzione superiore, percependo una distanza sociale fra operai e intellettuali. Questa fabbrica era appartenuta alla Rába per un certo periodo; Ede Horváth ricorda la sua lotta con la "gente di Budapest", che avrebbe voluto disciplinare ma che invece gli resisteva: il segretario del partito locale fu invitato a partecipare a un incontro in cui gli operai esposero le loro rimostranze nei confronti del manager e chiesero riparazioni. Horváth ricorda la vicenda come esempio del sacrificio degli interessi dell'efficienza economica, da lui difesi, a quelli politici (E. Horváth, Én volnék cit.).
} 
facevano distinzioni fra capitalismo occidentale, globalizzazione e capitalismo "nazionale", né pensavano che l'universalmente aborrito regime di Honecker rappresentasse una speciale via tedesca orientale alla modernità. Fra le eredità positive della Ddr, essi annoveravano istituzioni e prassi sociali che potevano essere facilmente incorporate nelle nuove ideologie di sinistra: il concetto di responsabilità sociale, il rafforzamento delle comunità, una maggiore solidarietà e un incremento della reciprocità nella vita collettiva. Questa "identità" tedesca orientale - se concepita come aperta ai valori comunitari e meno incentrata sul consumo rispetto al più materialista ovest - poteva essere facilmente conciliata con un sistema di valori postmaterialisti, peraltro nettamente alternativo al materialistico regime di Honecker. È per questa ragione che molti intervistati si dichiaravano «d'accordo» (einverstanden) con parole d'ordine quali consapevolezza ambientale, sviluppo sostenibile e maggiore responsabilità sociale. I tedeschi dell'est non criticavano la globalizzazione; al contrario, molti operai pensavano che le multinazionali avessero creato nuovi posti di lavoro e che avessero portato a Jena capitali e innovazioni. Essi giudicavano positivamente il multiculturalismo della vita universitaria e l'arrivo a Jena di studenti stranieri ${ }^{40}$; alcuni criticavano soltanto i Deutschrussen (tedeschi che vivevano nell'ex Urss e ai quali la Germania aveva conferito la cittadinanza) ${ }^{41}$. L'educazione antifascista giocò un ruolo importante nella mentalità politica e sociale di questa fascia d'età: tutti ritenevano la guerra la più terribile delle eventualità e pensavano che il genere umano dovesse evitarla ad ogni costo (la stragrande maggioranza di questi era nata dopo la seconda guerra mondiale) e anche gli anticomunisti convinti rifiutavano di paragonare la dittatura nazista con il regime di Honecker, ritenendo la prima molto più mostruosa.

Le opinioni sulla Germania ovest variavano all'interno dei gruppi di intervistati; in generale, tuttavia, $i$ tedeschi dell'est erano maggiormente consapevoli di cosa significasse trovarsi in una periferia capitalista di quanto non lo fossero gli ungheresi. Molti ammettevano che prima della Wende si erano sentiti inferiori ai tedeschi dell'ovest a causa della forte influenza delle rappresentazioni stereotipate del capitalismo (gli operai occidentali sarebbero stati meglio formati, più creativi, diligenti e motivati di quelli orientali impiegati nelle imprese di stato, che venivano considerati all'ovest meno disciplinati e più "indottrinati" ${ }^{42}$. Negli anni del post-socialismo tali stereotipi si

\footnotetext{
40 Jena è sede della famosa Friedrich-Schiller-Universität, che accoglie numerosi partecipanti al programma Erasmus e studenti da ogni parte del mondo.

${ }^{41}$ Tutti gli intervistati tedeschi orientali sapevano di star parlando con una cittadina ungherese, ed è improbabile che coloro che avevano forti sentimenti nazionalistici abbiano partecipato.

42 A tale proposito, alcuni intervistati mi hanno esplicitamente detto che non avrebbero parlato con un ricercatore della Germania ovest, a causa dei pregiudizi reciproci: il fatto che anch'io provenissi da un paese socialista è stato un vantaggio, poiché l'Ungheria era considerata un paese "amico" e "liberale", dove i tedeschi dell'est potevano incontrare il loro parenti dell'ovest. Il "liberalismo" del Partito comunista ungherese era constatato anche dai funzionari della Sed (Sozialistische Einheitspartei Deutschlands: Partito socialista unitario di Germania).
} 
modificarono, e i tedeschi orientali divennero maggiormente critici nei confronti del capitalismo; a loro parere, se pure la loro tecnologia non era avanzata quanto quella occidentale, le competenze erano paragonabili, e anzi essi dovevano essere più creativi dei loro omologhi dell'ovest proprio a causa delle deficienze tecniche (ad esempio, se una macchina si rompeva, dovevano essere in grado di ripararla mentre i tedeschi occidentali si limitavano a chiamare la manutenzione). La maggioranza era scettica circa le prospettive di raggiungere rapidamente lo stesso livello della Germania ovest e riteneva che ciò sarebbe avvenuto in non meno di 20-30 anni. Essi ritenevano tuttavia che la distinzione fra Wessi e $\mathrm{Ossi}^{43}$, per loro abituale, sarebbe scomparsa nella generazione successiva:

Nella Ddr l'operaio era allo stesso livello [sociale] del suo capo. Non riesco a immaginare un [capo] Wessi per il quale fosse lo stesso. Avevo una zia, che era emigrata in Germania ovest da giovane ed aveva sposato un tedesco occidentale. Vedeva le cose diversamente, ci guardava dall'alto in basso. Non le interessavano le nostre cose, se qualcosa era Ossi, per lei non andava abbastanza bene. Ci mandò un pacco, una volta, che riempì di polvere per dolci e vestiti di seconda mano, perché erano troppo buoni per dei poveri Ossi. Non volevo nemmeno sentire parlare di questa zia. Dopo la Wende andammo anche noi all'Ovest, e trovammo persone semplici, simili a noi - era del tutto diverso, erano amichevoli e non ci guardavano dall'alto in basso. Ci scambiammo regali, e ci ospitarono a casa loro - in breve, fu un rapporto completamente diverso da quello con questa $\mathrm{zia}^{44}$.

Come mostra chiaramente questa storia, il senso di inferiorità era spesso alimentato da esperienze umilianti con i tedeschi dell'ovest. Ciononostante, tutti gli intervistati ritenevano che l'unificazione fosse stata positiva per la "nazione" e che i loro figli non avrebbero conosciuto l'intensa propaganda che ciascun stato tedesco conduceva contro il sistema sociale dell' "altra" Germania. Le operaie raccontavano di quando i tedeschi dell'ovest credevano che i bambini dell'est all'asilo imparassero solamente canzoni da parata comunista e che venissero indottrinati fin dalla più tenera età. Una delle ragioni per cui molti tedeschi dell'est fecero propri i valori post-materialistici affondava le radici in questo senso di inferiorità: volevano dimostrare all'“ovest" che c'erano cose che i tedeschi orientali facevano meglio di quelli occidentali. La politica femminile, il sistema educativo e quello sanitario si trovavano in cima alla lista delle istituzioni di cui si parlava positivamente. Le madri affermavano all'unanimità che i loro figli avevano ricevuto maggiori attenzioni

\footnotetext{
${ }^{43}$ Nomignoli spregiativi per indicare rispettivamente i tedeschi dell'ovest e dell'est.

${ }^{44}$ Intervista del 2003 con Martha (52 anni), contabile, che aveva iniziato come operaia specializzata alla Zeiss e, dopo un corso di formazione, divenuta lì contabile. Perso il posto nel 1991, provò vari lavoretti (operatrice in un call center, libraia, piscicultrice, venditrice di Tupperware e di confezioni). Affermava che la sua molla principale non erano i soldi - suo marito guadagnava bene - ma il desiderio di stare in compagnia. Infine, trovò lavoro come contabile nella stessa azienda dove lavorava il marito.
} 
nel sistema scolastico della Germania est che dopo la Wende, e aggiungevano che nella Ddr i maestri prestavano maggior attenzione ai bisogni dei singoli bambini ed investivano maggiori energie nell'educazione degli alunni con voti al di sotto della media. Molte delle intervistate avevano preso parte a uno speciale programma educativo, il Frauensonderklasse (scuola delle donne), e ricordavano positivamente l'incontro con altre donne che si trovavano nella loro stessa situazione di madri lavoratrici con figli piccoli. Le reti di relazioni che erano state stabilite nelle Frauensonderklasse continuavano ad avere un ruolo significativo per queste donne, nonostante la maggior parte di loro avesse perso il proprio posto alla Zeiss.

L'ultima questione da esaminare è la valutazione soggettiva della rappresentanza degli interessi dei lavoratori dopo il cambio di regime. Gli ungheresi erano unanimemente pessimisti al riguardo: alla Rába operavano diverse sigle sindacali, che frammentavano le adesioni ed erano ritenute impotenti e incompetenti dagli stessi rappresentanti sindacali intervistati. Alla Zeiss, il presidente del consiglio aziendale riteneva che le rappresentanze dei lavoratori funzionassero efficacemente nelle grandi aziende quali la Zeiss stessa, ma era convinto che le piccole e medie imprese spesso vietassero (informalmente) l'adesione al sindacato e che i lavoratori non protestassero pur di mantenere il lavoro. Così egli valutava retrospettivamente il regime di Honecker:

La Ddr era uno stato di operai e contadini. I lavoratori avevano delle certezze ed erano liberi di studiare. Avevano più opportunità di studiare di quante ne avessero $i$ figli degli ingegneri e degli impiegati. E in fabbrica c'era un solida cultura comunitaria. Volevano fare vedere che le persone sono uguali, quindi la gerarchia aziendale era meno visibile. Non c'erano differenze nei contatti fra le persone: potevi parlare a chiunque alla stessa maniera, senza badare se fosse un operaio, un caporeparto o un dirigente. Le persone inserite in questo sistema trovano più difficile accettare che $\mathrm{i}$ manager siano separati dagli operai [...] Per me, la questione decisiva sono le possibilità che un uomo ha nella sua vita, quanto può influire sulla propria crescita personale. E credo che nel nuovo regime un uomo abbia più possibilità ${ }^{45}$.

Vi è una differenza cruciale nel giudizio sul cambio di regime tra i tedeschi dell'est e gli ungheresi. Anche il senzatetto Jan sarebbe stato d'accordo con il presidente del consiglio d'impresa della Zeiss sul fatto che un uomo aveva più possibilità nel nuovo sistema che nel vecchio. Gli ungheresi, al contrario, avvertivano che il nuovo regime aveva tolto loro anche gli obiettivi limitati (un appartamento, una casa, una gita nel week-end e vacanze regolari) che potevano raggiungere sotto Kádár.

Ciò spiega l'ambivalente giudizio sul regime di quest'ultimo. L'immagine di una maggiore uguaglianza sociale e materiale si intreccia con quel desiderio di ordine, di uno stato forte e di un governo autoritario riscontrato in mol-

\footnotetext{
45 Intervista con Thorsten (52 anni), presidente del consiglio d'impresa alla Zeiss, 2003.
} 
te interviste. Mentre gli intervistati tedeschi si identificavano con la Wende e nemmeno i disoccupati si auguravano un ritorno del regime di Honecker, solo pochi ungheresi pensavano di aver tratto benefici dal cambio di regime e dalla nuova democrazia. A causa delle esperienze negative vissute, che alimentavano le «narrazioni del declino», la maggioranza degli ungheresi era ostile al capitalismo "occidentale" e pensava che uno stato forte e una via ungherese alla modernità costituissero la panacea per i mali prodotti dalla propria condizione di periferia capitalistica. Mentre il maggior successo della Germania est nell'integrarsi nel mondo capitalista fu accompagnato da un cambiamento di mentalità e dalla comparsa di valori post-materialisti, in Ungheria il nazionalismo sembrò essere la sola alternativa a un capitalismo che aveva deluso e concretamente impoverito ampie fasce sociali.

È importante ricordare che le interviste sono state condotte fra il 2002 ed il 2004. In base ai risultati qui esposti, sarebbe stato possibile prevedere (o almeno spiegare) il successo elettorale di Fidesz, il partito di destra al governo in Ungheria, che ha ottenuto una maggioranza dei $2 / 3$ dei seggi parlamentari alle elezioni del 2010 e utilizza slogan quali lo stato forte, la punizione della corrotta élite ex-comunista e una speciale "terza via" al capitalismo. Tuttavia, alcune indagini fanno pensare che molti siano rimasti disillusi dalla ricetta "nazionale" e dall'estrema destra. Fidesz ha perso popolarità, anche perché l'aggressiva retorica sulla libertà e sul nazionalismo nulla ha prodotto per le famiglie, il cui livello di vita è spesso peggiorato rispetto a due anni fa; e molti giovani (che possono finalmente sfruttare le opportunità offerte dall'Unione Europea, fra cui gli scambi studenteschi e la libertà di spostamento) rifiutano le idee razziste di Jobbik.

(traduzione di Daniele Pipitone) 


\title{
Città nuove, colonizzazione e impero. Il caso di Fertilia
}

\author{
Marcel Farinelli
}

\begin{abstract}
New towns, colonization and empire. The case of Fertilia examines the reclamation project of a marsh area in the north-west of Sardinia, a plain called Nurra, near the city of Alghero, that was planned during the fascist regime. The project included the building of a new town, Fertilia, and the colonization of the entire area exclusively by peasants from the province of Ferrara. The marsh area was under the administration of the Alghero city administration, the nearest urban centre, that was also the only Catalan speaking minority in Sardinia and Italy. Analyzing the case study of the new town centre (Fertilia) created in opposition to the old town (Alghero), the author argues that the phenomenon of the new towns in Italy during the fascist regime was a way to definitively italianize the population of peripheral areas.
\end{abstract}

Keywords: Alghero, Sardinia, Italian fascism, internal colonization, italianization, Italian imperialism, new town

Parole chiave: Alghero, Sardegna, fascismo, colonizzazione interna, imperialismo italiano, città nuova

Per tutta la durata del regime fascista, furono realizzati in Italia, sia sul territorio nazionale che nelle «terre d'oltremare», vari nuclei urbani caratterizzati da una nuova concezione dell'architettura e del disegno, con dimensioni e funzioni ben diverse tra loro. Questi centri, definiti come città, borghi o villaggi a seconda delle loro dimensioni, furono costruiti per diversi motivi: alcuni per favorire lo sfruttamento di materie prime, come Carbonia in Sardegna o Arsa (oggi Raŝa) in Istria, altri per motivi strettamente militari, come Guidonia nel Lazio. Altri furono realizzati come parte integrante di un territorio che, un tempo malarico e incolto, era stato trasformato in terreno fertile e ospitale con una serie di interventi di bonifica. Ma aldilà delle città dell'Agro Pontino (Pomezia, Littoria/Latina, Sabaudia, Aprilia e Pontinia), sappiamo poco su quella che fu un'imponente opera di trasformazione del territorio e di population management, tanto che non esiste un censimento completo di questi insediamenti ${ }^{1}$. Nell'Italia del dopoguerra la ricerca storica

${ }^{1}$ F. Caprotti, Mussolini's Cities. Internal Colonialism in Italy, Cambria UP, Cambria 2007; D.Y. Ghirardo, Building New Communities. New Deal America and Fascist Italy, Princeton

«Passato e presente», a. XXXI (2013), n. 88 
si è concentrata quasi esclusivamente su alcuni aspetti, come il generale fallimento dei progetti, il loro carattere autoritario o le loro caratteristiche tecniche, senza però analizzare a fondo questo fenomeno ${ }^{2}$. A partire dalla seconda metà degli anni '90, la questione è stata spesso affrontata in chiave nostalgica e per lo più fuori dagli ambienti accademici, esagerando gli aspetti apparentemente "positivi", come l'aumento della disponibilità di terre coltivabili, la lotta alla malaria, o il raggiungimento dell'autarchia economica ${ }^{3}$, diffondendo un'immagine edulcorata di tali vicende, in particolare attraverso i circuiti televisivi ${ }^{4}$. Tutto ciò ha fatto sì che il programma di colonizzazione interna e di bonifica nel quale il regime fascista spese notevoli energie sia oggi visto come rivolto esclusivamente a risolvere problematiche economico-sociali o a generare consenso, se non addirittura come una sorta di riforma agraria a scapito dei grandi proprietari terrieri.

Vorrei suggerire, esaminando un caso specifico, che le opere di bonifica e la fondazione di nuovi centri non avevano un carattere esclusivamente economico o tecnico-agrario, ma rispondevano anche alla logica del "fare gli italiani" propria del fascismo e, in definitiva, alle pretese imperiali e totalitarie del regime. Secondo quanto suggerisce Robert O. Paxton, infatti, il fascismo è una

forma di condotta politica caratterizzata da un'ossessiva ansia di declino, umiliazione e vittimizzazione della comunità nazionale e da un compensativo culto dell'unità, della forza e della purezza, in cui un partito di massa di devoti militanti nazionalisti, agendo in scomoda ma efficace collaborazione con le tradizionali classi dirigenti, abbandona le libertà democratiche e persegue con redentoria violenza, svincolato da qualsiasi remora morale o giuridica, obiettivi di pulizia interna e di espansione territoriale 5 .

Per ottenere questa difesa della «comunità nazionale» e questa sua estensione, tanto interna come esterna, uno degli strumenti fu la fondazione di nuovi centri urbani, o la trasformazione radicale di quelli già esistenti, allo scopo di assorbire la comunità preesistente o di neutralizzarne le differenze

UP, Princeton 1989; E. Lo Sardo, Divina geometria. Modelli urbani degli anni venti, Maschietto\&Musolino, Firenze 1995; L. Nuti-R. Mariani, Le città di strapaese. La politica di fondazione nel ventennio, FrancoAngeli, Milano 1981; R. Mariani, Fascismo e "città nuove", il Mulino, Bologna 1976; D. Pizzi, Città metafisiche. Città di fondazione dall'Italia all'oltremare. 1920-1945, Skyra, Ginevra 2005. Per un censimento approssimativo, che arriva a 147 tra «città nuove», «borghi» e «villaggi», cfr. A. Pennacchi, Fascio e martello. Viaggio per le città del Duce, Laterza, Roma-Bari 2008, pp. XV e 287-95.

${ }^{2}$ Cfr. D.Y. Ghirardo, Building New Comunities cit, , pp. 20-22.

3 T. Stabili, La bonifica di Mussolini. La bonifica fascista dell'Agro Pontino, Settimo Sigillo, Latina 2002. In questo caso l'A., a scanso equivoci, dedica il libro «a Benito Mussolini che volle e realizzò la bonifica dell'Agro Pontino».

${ }^{4} \mathrm{Si}$ veda la fiction Questa è la mia terra, trasmessa su Canale 5 nella primavera del 2006.

${ }^{5}$ R.O. Paxton, Anatomy of Fascism, Knopf, New York 2004, p. 218 (trad. it. Mondadori, Milano 2005). 
rispetto a un canone di "italianità". Si tratta di una politica applicata in larga scala in ambito imperiale, e ne sono un esempio le ristrutturazioni urbanistiche di Asmara e Rodi che, con una fusione tra tradizioni locali, architetture italiane e art déco, furono trasformate in città che, sia pure con un certo esotismo, rispecchiavano quell'italianità codificata dal fascismo come "autentica" 6 . Durante la seconda guerra mondiale, poi, questa politica di italianizzazione e fascistizzazione fu applicata radicalmente nei vari territori della "comunità imperiale", con il tentativo di rimodellare etnicamente le zone occupate ed annesse. In questo contesto è particolarmente interessante il caso della Dalmazia, dove si applicò coscientemente il principio di affiancare agli autoctoni serbi e croati non espulsi comunità di italiani, allo scopo di «snazionalizzare» i primi e di assimilarli progressivamente ${ }^{7}$. A partire da queste riflessioni, e tenendo conto della differenza che il contesto nazionale rappresenta rispetto a quello coloniale, soprattutto in relazione al margine di manovra per poter applicare soluzioni drastiche, è possibile stabilire un nesso tra la colonizzazione interna, l'imperialismo e l'insieme delle politiche messe in campo dal regime per trasformare gli italiani in una stirpe di dominatori, nei «romani della modernità», per usare un termine caro a Mussolini. Queste politiche, naturalmente, erano una parte fondamentale dello sforzo mussoliniano di «fare gli italiani».

Ho preso ad esempio la «città nuova» di Fertilia, attualmente una frazione del comune di Alghero, che fu progettata nel contesto dei lavori di bonifica della principale piana del nord Sardegna (la Nurra), nella quale fu progettato l'insediamento di centinaia di famiglie provenienti, quasi esclusivamente, dalla provincia di Ferrara. Le opere, iniziate nel 1933, vennero interrotte durante la seconda guerra mondiale, ultimate dopo la fine del conflitto, in un clima politico ben diverso. Ciononostante, il caso di Fertilia è particolarmente adatto ad esporre la tesi proposta se si considerano le peculiarità storico-linguistiche del comune nel quale si trova, Alghero, nel quale risiede l'unica minoranza catalanoparlante in Italia. Le origini di questa comunità alloglotta risalgono al 1354, quando la Corona d'Aragona, nell'ambito della lunga e difficile conquista dell'isola, entrò definitivamente in possesso della città, un punto chiave nel sistema di potere della Repubblica di Genova nel nord Sardegna, e la ripopolò esclusivamente con sudditi provenienti dalla penisola iberica o dalle isole Baleari. La cittadina divenne un enclave catalano nel nord Sardegna, una caratteristica che ha mantenuto malgrado - a partire già dal XVI secolo - le minori difficoltà a ottenere la "cittadinanza" al-

${ }^{6}$ E. Denison-G. Yu Ren-N. Gebremedhin, Asmara. Africa's Secret Modernist City, Merrel, London-New York 2006; M. Fuler, Italy's Colonial Architecture and Urbanism. 1923-1940, «Cultural Anthropology», 3 (1988), n. 4, pp. 455-87; E. Lo Sardo (a cura di), Modelli urbani degli anni trenta cit.

7 D. Rodogno, Il nuovo ordine mediterraneo. Le politiche di occupazione dell'Italia fascista in Europa (1940-1943), Bollati Boringhieri, Torino 2003, pp. 332-35. 
gherese $^{8}$. L'uso di questo idioma da parte della comunità algherese, nonostante l'importante influsso di altre lingue - il sardo prima e l'italiano poi -, si è mantenuto anche dopo il passaggio dell'isola alla dinastia Savoia (1720) e l'unificazione della penisola, due fattori che hanno comportato l'arrivo in città di numerosi nuovi abitanti, in buona parte commercianti liguri e pescatori di corallo corsi o napoletani. La sopravvivenza del catalano di Alghero è stata possibile soprattutto perché questo idioma, ben diverso del sardo parlato da chi viveva fuori città, rappresentava un elemento di integrazione per i nuovi arrivati (o almeno per quelli che lo apprendevano) e allo stesso tempo un modo per marcare la differenza con quanti non erano ammessi ai privilegi della cittadinanza ${ }^{9}$.

Il fenomeno di una lingua che, nonostante la fine di ogni relazione culturale, istituzionale o commerciale, sopravviveva al passare del tempo e dei diversi governi che guidarono la Sardegna suscitò, a partire della seconda metà del XIX secolo, la curiosità sia della nascente dialettologia italiana, che di intellettuali iberici vicini al nazionalismo catalano. A partire dal 1864 si avviarono i primi contatti culturali con la Catalogna, intensificati dopo il soggiorno nella cittadina sarda di Eduard Toda i Güell, viceconsole spagnolo a Cagliari, che promosse la "catalanità" di Alghero, sia favorendo un movimento culturale che si esprimesse nell'idioma locale, sia divulgando questa realtà presso un pubblico catalano. Sempre negli anni '80 del XIX secolo alcuni studiosi italiani di dialettologia - Giuseppe Morosi, Pier Enea Guarnerio - dimostrarono che ad Alghero si parlava una variante del catalano (detto algherese o catalano di Alghero) ${ }^{10}$. La cittadina sarda entrò così nell'immaginario del movimento nazionalista catalano, che pretendeva di ridefinire la relazione con uno Stato spagnolo in evidente crisi ${ }^{11}$. In realtà solo

${ }^{8}$ B. Anatra, Dall'unificazione aragonese ai Savoia, in G. Galasso (a cura di), Storia d'Italia, vol. IX, Utet, Torino 1984, pp. 228-56; R. Conde, Il ripopolamento catalano di Alghero, in A. Mattone-P. Sanna (a cura di), Alghero, la Catalogna, il Mediterraneo, Gallizzi, Sassari 1995, pp. 75-103; G. Meloni, Genova e Aragona all'epoca di Pietro il Cerimonioso, vol. I, Cedam, Padova 1971, pp. 149-214.

${ }^{9}$ R. Caria, El català a l'Alguer: apunts per a un llibre blanc, «Revista de Llengua i Dret», 2006, n. 46, pp. 42-47. Sulla minoranza algherese, cfr. E. Blasco i Ferrer, Il dialetto catalano di Alghero, in J. Carbonell-F. Manconi (a cura di), I Catalani in Sardegna, Silvana Editoriale/Consiglio Regionale della Sardegna, Cagliari 1984, pp. 167-70; R. Caria, Introduzione, in E. Toda i Güell. L'Alguer, un popolo catalano d'Italia, Gallizzi, Sassari 1981, pp. 9-76 (ed. or. Barcelona 1888).

10 P.E. Guarnerio, Il dialetto catalano di Alghero, «Archivio glottologico italiano», 9 (1886), pp. 261-364; G. Morosi, L'odierno dialetto di Alghero in Sardegna, in Aa.Vv., Miscellanea di filologia e linguistica: in memoria dei professori Napoleone Caix e Ugo Angelo Canello, Firenze 1886, pp. 312-33.

${ }^{11}$ Sul nazionalismo catalano cfr. A. Ballcells, El nacionalismo catalan, Historia 16, Madrid 1991; A. Rovira i Virgili, Els corrents ideològics de la Renaixença catalana, Barcino, Barcelona 1966. Per una visione anticonformista, cfr. J. Pich i Mitjana, Valentí Almirall i el federalisme intransigent, Afers, Catarroja-Valencia 2006; E. Ucelay Da Cal, El imperialismo catalan. Prat de la Riba, Cambó, d'Ors i la conquista moral de España, Edhasa, Barcelona 2003. 
un piccolo gruppo di intellettuali algheresi caldeggiò apertamente la "patria" catalana, ricoprendo comunque il fondamentale ruolo di rappresentanti della reificazione del passato imperiale e dell'antico splendore della nazione. Questa importanza dipendeva, e in parte dipende ancora, dal fatto che ad Alghero, per una combinazione di diversi fattori, la lingua maggiormente utilizzata, sia dalla popolazione che dagli intellettuali catalanisti, rimase la variante locale del catalano fino almeno agli anni ' 70 del '900. A partire da allora, almeno secondo i maggiori studiosi della materia, il fenomeno migratorio unito a una scolarizzazione generalizzata e alla diffusione di radio e televisione, creando per la prima volta uno spazio comunicativo comune a tutti gli italiani, portarono all'affermazione quasi definitiva, nell'uso, della lingua italiana $^{12}$.

Tenendo conto di questi aspetti, bisogna considerare come il fascismo si presentò nell'isola: un fenomeno marginale e molto debole, con caratteristiche differenti da quello continentale, tanto che la storiografia è arrivata ad individuare una corrente esclusivamente isolana, il sardofascismo ${ }^{13}$. Nel caso di Alghero, poi, il fascismo appare da subito come un fenomeno estremamente circoscritto quasi esclusivamente a pochi esponenti della élite cittadina, e solo dopo la stabilizzazione del regime aumentano gli iscritti al partito. Nella cittadina, guidata da una giunta a forte presenza sardista, non si verificarono scontri violenti tra fascisti e antifascisti, nemmeno attorno alla questione linguistico-nazionale, soprattutto per la scarsa politicizzazione della valorizzazione dell'idioma locale da parte dei catalanisti. L'unico episodio da registrare è il furto della bandiera sarda esposta nella sala municipale, un atto ripetuto da fascisti e nazionalisti italiani in altri comuni sardi controllati dal Partito sardo d'azione ${ }^{14}$. Malgrado l'assenza di un contrasto forte su temi identitari, è comunque innegabile che le politiche avviate dal regime aspirassero, soprattutto attraverso un'accurata pedagogia ed una propaganda altrettanto incisiva, alla sostituzione definitiva del catalano di Alghero con l'italiano ${ }^{15}$.

12 R. Caria, Alghero. Lingua e società, Edes, Cagliari 1990, pp. 15-30.

${ }^{13}$ Cfr. Aa.Vv., Il fascismo nel Mezzogiorno e in Sardegna, Atti del convegno (Nuoro, 2627 giugno 1976), «Archivio sardo del movimento operaio, contadino e autonomistico», 1977, n. 8-10, pp. 3-106; S. Cubeddu (a cura di), Il sardo-fascismo tra politica, cultura ed economia, Atti del convegno (Cagliari, 26-27 novembre 1993), Fondazione Sardinia, Cagliari 1993; L. Marrocu, Il ventennio fascista (1923-1943), in L. Berlinguer-A. Mattone (a cura di), Storia d'Italia. Le regioni dall'Unità ad oggi, La Sardegna, Einaudi, Torino 1998, pp. 633-53; M.D. Picciau, Tra sardismo e fascismo. Arte e identità nelle riviste sarde del Novecento, Zonza, Cagliari 2007; S. Sechi, Dopoguerra e fascismo in Sardegna, Einaudi, Torino 1969; L.M. Plaissant (a cura di), La Sardegna nel regime fascista, Cuec, Cagliari 2000. Per una negazione della validità di questo concetto, cfr. G. Stogiu, Storia della Sardegna dalla grande guerra al fascismo, Laterza, Roma-Bari 1990 e Id., Storia della Sardegna durante il fascismo, Laterza, Roma-Bari 1995.

${ }^{14}$ Archivio storico del Comune di Alghero (d'ora in poi Asca), b. 1003, fasc. 59, c. 1.

${ }^{15} \mathrm{Sul}$ fascismo algherese cfr. M.A. Farinelli, Il fascismo ad Alghero. Italianizzazione alla periferia del regime, «Insula», 2009, n. 6, pp. 67-92; Id., El feixisme a l'Alguer, Angle Editorial, Barcelona 2010. 
Nell'analisi della colonizzazione e della bonifica del territorio algherese, bisogna quindi considerare queste caratteristiche, indispensabili per comprendere sia l'atteggiamento del regime davanti alla peculiarità storico-linguistica della città, sia le politiche, e le aspirazioni, legate alla colonizzazione interna.

\section{La politica di "fondazione” in Sardegna}

L'isola sarda è sempre stata, almeno in epoche storiche recenti, un territorio afflitto da una bassa densità abitativa e dal problema endemico della malaria, due fattori estremamente legati tra loro e che da molti sono stati individuati come elementi chiave per spiegare l'arretratezza dell'economia sarda. Durante gli anni '20 e '30 del '900 l'economista Gavino Alivia, sassarese e fascista in ottime relazioni con le gerarchie locali, dedicò buona parte delle sue energie intellettuali ad indagare la relazione tra popolazione e sviluppo economico dell'isola e a cercarvi soluzioni. Alivia sosteneva che la popolazione sarda «è scarsa, distribuita sul territorio in modo non rispondente alle esigenze dell'agricoltura, lontana dal mare, fisicamente debilitata, economicamente poco produttiva» ${ }^{16}$. L'economista sassarese, in realtà, ripercorreva un strada già battuta da altri, nel tentativo di promuovere lo sviluppo economico dell'isola attraverso il ripopolamento e la costruzione di nuovi centri urbani. La colonizzazione infatti, sia attraverso nuclei di popolazione isolana che attraverso immigrati, è stata un elemento costante nell'evoluzione della società sarda. A ben vedere, l'urbanizzazione nelle coste è sempre stato un fenomeno importato dalle varie talassocrazie che hanno esteso la loro influenza sulle coste sarde (fenici, cartaginesi, romani, pisani, genovesi, e catalani) e la stessa Alghero trae le sue origini da una prima colonizzazione genovese, seguita da quella ben più duratura e genericamente indicata come catalana.

Ma è da quando l'isola entra a far parte dei domini della dinastia Savoia che si susseguono le iniziative per "popolare" o "ripopolare" vaste aree della Sardegna, sia per motivi strettamente economici e legati al riformismo illuminato settecentesco, quanto per ragioni connesse al controllo del territorio ed alla lotta al banditismo, fenomeno endemico già in quegli anni ${ }^{17}$. I primi tentativi risalgono agli anni ' 30 del XVIII secolo, quando fu favorito, con un certo successo, l'insediamento nell'isola di S. Antioco di una colonia di genovesi provenienti dall'isola di Tabarca, davanti alle coste tunisine, i quali fondarono Carloforte (1738) e pochi anni dopo, nella vicina isola di S. Pietro,

16 G. Alivia, Economia e popolazione della Sardegna settentrionale, Gallizzi, Sassari 1931, p. 68. Si veda anche Id., La distribuzione della popolazione della Sardegna tra la montagna ed il litorale, Istituto Poligrafico dello Stato, Roma 1931 e La colonizzazione interna e la Sardegna, Poligrafica Universitaria, Firenze 1938.

17 Cfr. A. Mattone, Le origini della questione sarda. Le strutture, le permanenze, le eredità, in L. Berlinguer-A. Mattone (a cura di), Storia d'Italia cit., pp. 36-46. 
Calasetta (1769) ${ }^{18}$. A metà secolo le autorità sabaude continuarono ad incentivare la colonizzazione da parte di nuclei di popolazione non autoctona, e tra il 1746 ed il 1774 si cercò più volte di insediare, prima a S. Antioco, poi all'Asinara ed infine a Montresta nei pressi di Bosa, abitanti di origine greca. Questi provenivano da una fallita colonizzazione in Corsica (Paomia, nei pressi di Ajaccio), dalla quale erano fuggiti a causa dei contrasti con la comunità locale, e - sempre per lo stesso motivo - furono poi costretti ad abbandonare la località sarda ${ }^{19}$. Un altro esempio importante è quello della Gallura con le cosiddette "isole intermedie", che costituiscono oggi l'Arcipelago della Maddalena. La Gallura è considerata una microregione nell'isola, con dei legami antropici, economici e linguistici molto forti con il sud della Corsica che la rendono, assieme alle caratteristiche orografiche, un'area di confine caratterizzata dalla forte presenza del contrabbando e dall'essere un ottimo rifugio per banditi e fuorilegge di entrambe le isole ${ }^{20}$. Allo scopo di aumentare il controllo su questa area, nel corso del ' 700 la corte sabauda promosse lo stanziamento di allevatori corsi in casolari sparsi per il territorio (stazzi), favorendo anche la colonizzazione delle "isole intermedie" con popolazione di origine corsa e ligure 21 . Agli inizi dell' 800 , però, la situazione era ancora largamente fuori dal controllo delle autorità sabaude, e in un clima segnato dall'eco della Rivoluzione francese e dalla continua minaccia che il contagio rivoluzionario potesse arrivare in Sardegna dalla Corsica, territorio francese dal 1769 (a parte una breve parentesi sotto Paoli e la protezione britannica tra 1794 e 1797), si decise di fondare una nuova città. Era Santa Teresa di Gallura, sorta proprio nei pressi dell'approdo più vicino alla Corsica, centro dell'attività dei contrabbandieri e luogo chiave per il passaggio dei fuorilegge da una parte all'altra delle Bocche di Bonifacio 22.

Alla necessità di migliorare il controllo del territorio si aggiunse nel XIX secolo la volontà di favorire il progresso economico e sociale della Sardegna, grazie proprio all'eliminazione dei fattori che ne causavano l'arretratezza: la malaria e, in generale, la scarsità dei terreni coltivati. In particolare dopo l'abolizione del feudalesimo (1836) e l'unificazione, si ebbero così in Sarde962.

18 G. Vallebona, Carloforte. Storia di una colonizzazione, Tamburino Sardo, Carloforte

19 R. Ciasca, Corsi colonizzatori di terre sarde nel secolo XVIII, «Archivio storico di Corsica», 1928, n. 4, pp. 294-314; C. Moretti, Una colonia di greco-corsi in Sardegna: Montresta, Carbongemma, Sassari 1982; G. Piroddi, La colonia dei greci a Montresta (1750-1830), Gallizzi, Sassari 1967.

20 Cfr. S. Pira, La Gallura nel settecento. Una repubblica montanara tra contrabbando e banditismo, in Studi e ricerche in onore di Girolamo Sotgiu, vol. II, Cuec, Cagliari 1994, pp. 91-105.

21 G. Salice, Bonifica e ripopolamento della Gallura da Rivarolo a Garibaldi, pp. 91-97, in G. Continiello (a cura di), Garibaldi. Mille volti, mille vite, AM\&D, Cagliari 2010, pp. 91108.

22 S. Rattu, Santa Teresa di Gallura, in Studi storici in onore di Francesco Loddo Canepa, vol. I, Sansoni, Firenze 1959, pp. 253-309. 
gna vari progetti di bonifica, soprattutto delle aree pianeggianti e malariche vicino alle coste. Si trattava di iniziative promosse da privati, in parte legate a solidi progetti imprenditoriali, in parte maggiormente ispirate dall'ideale democratico e repubblicano del piccolo proprietario terriero; comunque, nonostante le premesse, furono circoscritte a pochi ettari di terreno. Tra i progetti più imponenti ricordo quello della società «La Giovane Icnusa» del 1853, e quello promosso dal conte Francesco Aventi nel 1869, sostenuto anche da Garibaldi ${ }^{23}$. I primi interventi di una certa importanza, per estensione e ricadute economiche, si ebbero però dopo che il governo, in seguito a forti agitazioni sociali, promulgò le prime leggi speciali per la Sardegna, che nel 1897 e poi nel 1907 misero a disposizione importanti incentivi per lo sviluppo economico. Fu in questa fase, a cavallo tra i due secoli, che anche in un territorio già interessato da un'intensa attività agricola come quello di Alghero si ebbero i primi interventi di bonifica della Nurra, sia a spese di privati tra cui le tenute vinicole de "i Piani" e l'azienda di Surigheddu - sia per iniziativa statale, come nel caso della Colonia penale di Cuguttu, proprio alle porte della città.

Quando il regime fascista si impegnò a portare a termine un'incisiva opera di bonifica nell'isola, si mosse dunque su di un terreno già ampiamente battuto. Ma per le risorse messe in campo nelle opere e per la serie di significati ideologici ad esse legate, non possiamo leggere la colonizzazione fascista nel solco della semplice continuità rispetto alle iniziative precedenti. Nella dottrina fascista, infatti, l'importanza della questione demografica era quasi ossessiva, come del resto lo era per buona parte della destra europea, in particolare da quando Oswald Spengler aveva pubblicato Der Untergang des Abendlandes, dove il nesso tra andamento demografico e potenza economico-militare di una nazione era direttamente proporzionale ${ }^{24}$. L'importanza data da Mussolini alla demografia, e naturalmente al razzismo, è stata recentemente oggetto di autorevoli studi, i quali hanno messo in luce le diverse politiche avviate per aumentare, e migliorare, la popolazione nazionale ${ }^{25}$. In questo contesto si afferma la cosiddetta "scuola italiana" di eugenetica, rappresentata da scienziati quali Nicola Pende, nella quale l'idea di una selezione delle nascite come mezzo per migliorare la stirpe viene sostituita dalla convinzione che un'adeguata assistenza medica durante la gravidanza e un controllo scientifi-

${ }^{23}$ Cfr. G. Salice, Bonifica e ripopolamento cit., pp. 100-04.

${ }^{24}$ O. Spengler, Der Untergang des Abendlandes, vol. II, C.H. Beck'sche Verlagsbuchhandlung, Monaco 1922, pp. 101-88 (trad. it. Longanesi, Milano 1957). Sull'influenza di Spengler in Italia durante il fascismo, cfr. P. Azzaro, Deutsche Geschichtsdenker um die Jahrhundertwende und ihr Einfluss in Italien. Kurt Breysig, Walther Rathenau, Oswald Spengler, Peter Lang, Berna 2005, pp. 601-89.

${ }_{25}$ C. Ipsen, Dictating Demography. The Problem of Population in Fascist Italy, Cambridge UP, Cambridge 1996 (trad. it. il Mulino, Bologna 1997); G. Israel-P. Nastasi, Scienza e razza nell'Italia fascista, il Mulino, Bologna 1998; A. Gillette, Racial Theories in Fascist Italy, Routledge, London-New York 2002; F. Cassata, Molti, sani e forti. L'eugenetica in Italia, Bollati Boringhieri, Torino 2006. 
co dello sviluppo e della procreazione avrebbero garantito elementi sani e una razza migliore. Il padre della endocrinologia sosteneva che, secondo le sue teorie conosciute con il nome di «ortogenesi», fosse possibile gestire al meglio quello che definiva «capitale umano nazionale», selezionando fin dai primi anni di vita i soggetti validi per poterne favorire lo sviluppo come ottimi lavoratori e soldati, ed escludendo $\mathrm{i}$ «mediocri nel corpo e nello spirito» 26 .

Un aspetto importante di questo interesse fascista per la popolazione era rappresentato dalla politica di colonizzazione e migrazione interna, soprattutto durante gli anni '30. Le prime esperienze furono realizzate proprio in Sardegna, l'area più spopolata del paese, dove nell'estremità nord del Campidano agli inizi degli anni '20 un gruppo privato, la Società Bonifiche Sarde, aveva intrapreso un vasto progetto che portò alla realizzazione della «città nuova» di Mussolinia (oggi Arborea). La crisi del '29, però, portò alla paralisi delle opere, e come in altri ambiti dell'economia italiana la situazione si risolse con l'intervento dello Stato, che si fece carico di terminare i lavori e, naturalmente, di dargli un'impronta decisamente fascista (la Casa del fascio e quella del Balilla furono aggiunte solo nel 1934) ${ }^{27}$. Il modello inaugurato a Mussolinia fu sviluppato e applicato in larga scala durante gli anni ' 30 nella bonifica delle Paludi Pontine, dove l'estensione dei terreni, il numero di coloni e l'uso propagandistico che se ne fece resero questa bonifica la più conosciuta e spettacolare. L'interesse per queste paludi trasformate in terre coltivabili e per questi nuovi centri abitati era grande non solo tra urbanisti ed architetti, ma anche tra quanti si preoccupavano per «il problema dei problemi». Scienziati come Nicola Pende e Corrado Gini, o come il fisiologo Sabato Visco e l'antropologo Sergio Sergi, si precipitarono nell'Agro Pontino per studiare il comportamento demografico e lo sviluppo psicofisico delle famiglie di coloni, come se si trattasse di colture in vitro dei rappresentanti della nuova stirpe italiana ${ }^{28}$. L'importanza che la comunità scientifica italiana dava alla colonizzazione interna è ben dimostrata anche da un testo dello stesso Pende nel quale si istituiva un parallelo tra la bonifica agricola e la «bonifica umana razionale», ovvero l'applicazione delle sue teorie di population management che, se applicate, avrebbero purgato la società dagli elementi nocivi e garantito che ad ogni individuo fosse assegnata la funzione sociale adatta alla sua costituzione fisica e psichica ${ }^{29}$.

Tutta questa serie di aspirazioni sociali, scientifiche e politiche caratterizzarono fortemente il fenomeno della bonifica e della fondazione di nuovi centri urbani, sia entro i confini nazionali che nei possedimenti coloniali, do-

\footnotetext{
${ }^{26}$ G. Israel-P. Nastasi, Scienza e razza nell'Italia fascista cit., pp. 136-41.

27 M.L. Di Felice, L'ascesa delle aziende edili all'ombra delle bonifiche e dei lavori pubblici, in L.M. Plaisant (a cura di), La Sardegna nel regime fascista cit., pp. 83-113; L. Marrocu, Il ventennio fascista, in L. Berlinguer-A. Mattone (a cura di), Storia d'Italia cit., pp. 67585.

${ }^{28}$ G. Israel-P. Nastasi, Scienza e razza nell'Italia fascista cit., pp. 152-55.

${ }^{29}$ N. Pende, Bonifica umana razionale e biologia politica, Cappelli, Bologna 1933.
} 
ve certi aspetti furono sicuramente più accentuati. Queste «città nuove», concepite come il centro attorno al quale gravitavano la bonifica e la colonizzazione, dovevano essere i luoghi ideali per realizzare queste utopie eugenetiche e sociali, ed erano pertanto profondamente diverse da quel «tipo di urbanesimo che è distruttivo, che isterilisce il popolo, ed è l'urbanesimo industriale» ${ }^{30}$. I nuovi centri, in sostanza, erano lo strumento principale per preservare, attraverso la liturgia della «ruralità» e della «romanità», i valori tradizionali e nazionali della società, ed erano allo stesso tempo il laboratorio, $o$ nucleo, della nuova società fascista.

\section{La trasformazione del territorio}

La Sardegna, nell'ambito della colonizzazione interna, sembrava offrire diverse opportunità: era un'isola grande come la Sicilia ma con bassa densità abitativa e la disponibilità di discrete aree pianeggianti come il Campidano, a sud, e la Nurra, a nord. Inoltre, la scarsa antropizzazione del territorio, in parte legata all'economia prevalentemente pastorale, aveva favorito l'espandersi delle paludi costiere, con il risultato che l'isola - l'antico "granaio" di Roma - era ancora negli anni ' 30 una delle aree più afflitte dalla malaria dell'intero bacino mediterraneo. Il regime fascista così si concentrò, dopo una prima bonifica nel Campidano, sulla Nurra. Compresa negli attuali comuni di Alghero, Sassari, Porto Torres, Stintino e Olmedo, la Nurra era in gran parte coperta da una fitta macchia mediterranea ed abitata prevalentemente da pastori che vivevano ancora in case-ovili di pietra e paglia (sa pinnetta, in sardo). La parte compresa nel municipio algherese, inoltre, era caratterizzata da uno stagno di medie dimensioni, il Calik, e da un sistema di paludi e acquitrini che rendevano l'area malsana e quasi disabitata. Gli unici segni di civiltà erano gli edifici di una colonia penale alle porte di Alghero (Cuguttu), dove i carcerati lavoravano alcuni terreni appena bonificati, un vecchio ponte medievale che attraversava lo stagno e qualche azienda agricola. La desolazione era notevole, come testimoniano le memorie di Alfred Steneizer, geografo, storico dell'arte ed ex-ufficiale austroungarico che durante i primi anni '20 intraprese un viaggio in Sardegna. Mentre si spostava da Sassari ad Alghero, Steneizer osservava stupito il paesaggio:

la regione tra Sassari, Porto Torres ed Alghero [...] è di una monotonia opprimente. [...] Assomiglia ad una steppa, erba magra e secca, asfodeli e palme nane che per miglia e miglia costituiscono l'unica copertura del suolo. [...] È una sensazione di liberazione quando il treno scende tra gli oliveti verso il mare e si fanno visibili le onde azzurre che bagnano i bastioni e le torri di Alghero ${ }^{31}$.

${ }^{30}$ B. Mussolini, Discorso dell'ascensione, Libreria del Littorio, Roma 1927, pp. 21-22.

31 A. Steinitzer, L'isola dimenticata. La Sardegna e i sardi, in S. Govoni, La Sardegna descritta da Alfred Steinitzer (1924). Lo sviluppo della città di Alghero da allora ad oggi, Edizioni del Sole, Alghero 1998, pp. 38-39. 
La necessità di bonificare questa area, ricordata già a fine ' 800 , divenne un cavallo di battaglia dei sardisti e poi dei sardofascisti locali. Alcune iniziative, promosse da proprietari terrieri, furono realizzate anche con un relativo successo, ma per un intervento in grande scala che interessasse lo stagno ed i suoi dintorni paludosi si dovettero attendere gli sforzi fatti durante il periodo in cui era podestà di Alghero Paolo Enrico ${ }^{32}$.

Già eletto sindaco con il voto dei sardisti nel 1920 e poi passato al fascismo nel 1923, Enrico restò in carica fino al 1932, rappresentando una certa continuità tra i due movimenti, come è peculiare del fascismo isolano. La sua scomparsa dalla scena politica avvenne dopo qualche anno di alternanza con diversi commissari prefettizi, il che alimenta il sospetto di un conflitto in seno al fascismo locale. Durante la sua carica i tentativi promossi o comunque appoggiati dal Comune per realizzare opere di bonifica non dettero molti frutti, sia per la mancanza di fondi che per l'opposizione della locale Federazione provinciale degli agricoltori fascisti e del Comitato promotore per la costituzione di un consorzio di bonifica, al quale peraltro il Comune aveva deciso di partecipare ${ }^{33}$. Il Comitato si costituì a Sassari nel 1925 per iniziativa dei proprietari terrieri (ovvero il blocco sociale contrario alle istanze sardiste di riforma agraria) e dopo l'approvazione nel 1928 della «legge Mussolini» sulla bonifica integrale si attivò per evitare che la trasformazione del territorio comportasse eccessivi cambiamenti nell'assetto fondiario. Per questo motivo, il Comitato ostacolò le iniziative del Comune, onde evitare che dopo la bonifica della vasta area paludosa di sua proprietà i terreni fossero assegnati agli ex combattenti della Brigata Sassari, intaccando ovviamente gli interessi dei grandi proprietari terrieri. Questi si opposero in particolare al progetto elaborato dall'ingegnere algherese Fausto Cella il quale, con fondi pubblici e tramite una ditta di Sassari, intendeva trasformare la piana in un'area dove produrre materie prime da trasformare poi nelle industrie alimentarie cittadine. Il progetto teneva conto della disoccupazione locale e della necessità di crescita economica ed urbana di Alghero, e non a caso univa tutta la nuova area agricola alla città mediante una fitta rete stradale, mentre non vi era traccia di nuovi nuclei urbani ${ }^{34}$. Malgrado questi sforzi, il progetto venne rinviato per mancanza di fondi, e al podestà non rimase che chiedere, invano, l'intervento del governo ${ }^{35}$.

Durante i primi anni ' 30 però, per una serie di motivi estranei alla realtà sociale ed economica del comune sardo, fu avviato nella Nurra algherese uno dei più impattanti progetti di trasformazione del territorio, ancora più ambizioso di quelli fino allora proposti. In un clima caratterizzato dalla radicaliz-

32 Cfr. Asca, b. 958 , fasc. 2 , cc. 1-2; b. 900 , fasc. 26, c. 1.

33 Asca, Deliberazioni del Comune, reg. 547, deliberazione n. 172 del 23 novembre 1928.

${ }^{34}$ E. Tognotti, L'economia e la società algherese tra le due guerre (1919-1938). La bonifica della Nurra e la nascita della "città nuova" di Fertilia, in A. Mattone-P. Sanna (a cura di), Alghero, la Catalogna, il Mediterraneo, Gallizzi, Sassari 1994, pp. 629-32.

35 Asca, Deliberazioni del Comune, reg. 549, deliberazione n. 76 del 14 maggio 1932. 
zazione del regime e dalle difficoltà economiche generate dalla crisi economica mondiale, si fece strada l'idea che la trasformazione delle terre paludose in poderi da assegnare a coloni provenienti dalle zone ad alta densità demografica ed alto tasso di disoccupazione avrebbe risolto in parte il problema. Così da una parte si contrastava la disoccupazione e dall'altra si cercava di raggiungere 1' «autarchia». In fin dei conti si trattava di un'idea già diffusa alla fine del ' 800 , consistente nel trasferire nuclei di popolazione da aree sovraffollate a terre vergini, recentemente riscattate, dove i nuovi coloni avrebbero acquistato la proprietà con il loro lavoro ${ }^{36}$. Si trattava della rivendicazione storica degli ex combattenti, ai quali nell'ultimo anno di guerra era stato fatto credere che la vittoria avrebbe comportato una distribuzione di terre.

Secondo alcuni autori che hanno analizzato la bonifica delle paludi Pontine, ultimata tra il 1931 e il 1941, questa non era semplicemente una opera di trasformazione del territorio legata a una logica economica, ma era un vasto progetto di ingegneria sociale ${ }^{37}$ : le cinque «città nuove» alle porte di Roma erano parte del progetto fascista di costruire una nuova civiltà e di realizzare i propri sogni imperiali ${ }^{38}$. Queste ambizioni denotavano la volontà di un controllo totalitario e di trasformare in senso fascista la stessa natura: «il fascismo percepiva la natura indisciplinata, non civilizzata ed improduttiva delle paludi "mortifere" come un qualcosa che era necessario eliminare dalla faccia dell'Italia, per realizzare una nuova, addomesticata, ideale natura fascista che avrebbe favorito l'interazione umana nel lavoro agricolo e nella vita rurale, e che avrebbe garantito la crescita di fascisti ideali»39.

Sono tutti questi elementi (costruzione del consenso, "gestione" della popolazione, aspirazioni imperiali e autarchia) che caratterizzarono anche la bonifica della Nurra. Per realizzarla fu creato il 5 luglio del 1933 l'Ente ferrarese di colonizzazione $(E f c)$, che avrebbe dovuto organizzare il trasferimento di centinaia di famiglie provenienti dalla provincia di Ferrara nella poco fascista piana algherese, ed allo stesso tempo occuparsi della bonifica, della costruzione degli alloggi e dei fabbricati agricoli, nonché dell'assistenza tecnica e finanziaria ai nuovi arrivati. Tutta l'operazione fu organizzata, dunque, a partire della provincia di Ferrara, dove la presenza di numerosi braccianti, combinata con gli alti tassi di natalità e gli effetti della crisi economica avevano creato una situazione pericolosa, tanto che nel promuovere la colonizzazione sembra abbia avuto un ruolo fondamentale anche Italo Balbo, probabilmente

\footnotetext{
36 R. Mariani, Fascismo e "città nuove" cit., pp. 60-72.

${ }_{37}$ C. Ipsen, Dictating demography cit., pp. 109-13; R. Mariani, Fascismo e "città nuove" cit., pp. 171-78.

38 S.B. Frandsen, "The war that we prefer": The Reclamation of the Pontine Marshes and Fascist Expansion, in G. Sørensen-R. Mallet (eds.), International Fascism. 1919-1945, Frank Cass, London-Portland 2002, pp. 68-81.

${ }^{39}$ F. Caprotti-M. Kailka, Producing the ideal fascist landscape. Nature, materiality and the cinematic representation of the Pontine Marshes, «Social\&Culture Geography», 9 (2008), n. 6, p. 629.
} 
pressato da una base che mal sopportava sia la lentezza con la quale proseguiva la "rivoluzione fascista" che la situazione economica ${ }^{40}$. La possibilità di realizzare queste opere in Sardegna era offerta dal legame tra Balbo e il sassarese Mario Ascione, dirigente sindacale agrario, prima a livello provinciale e poi nazionale, e deputato che, insieme ad altri fascisti sassaresi, aveva duramente contrastato i progetti di bonifica promossi dal Comune o dal movimento sardista negli anni precedenti ${ }^{41}$.

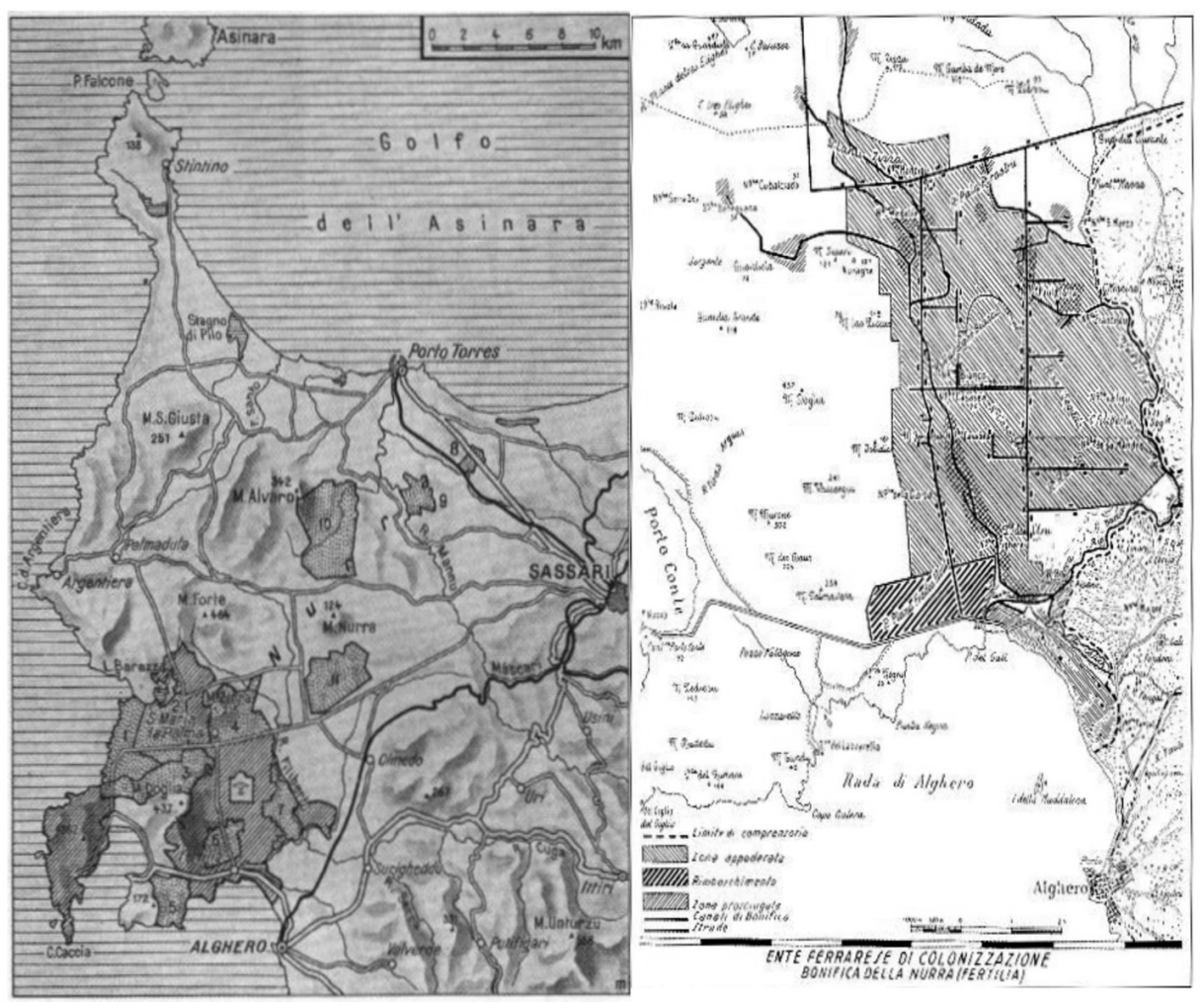

La bonifica della Nurra. A sinistra una veduta generale del comprensorio di bonifica, a destra lo stato dei lavori nel 1933. Si nota l'opposizione spaziale tra Fertilia ( $P$. del Gall nell'immagine) e Alghero. Fonte: G. Peghin, La colonia penale di Tramariglio, Carlo Delfino, Cagliari 2010, p. 13 (sinistra); A. Cucciola, Vecchie cittàlcittà nuove. Concezio Petrucci 1926-1946, Dedalo, Bari 2006, p. 257 (destra)

40 Testimonianza di A.F. (1930-), figlio di un dirigente dell'Efc e membro della Milizia volontaria per la sicurezza nazionale, trasferitosi ad Alghero durante la bonifica.

${ }^{41}$ M. Brigaglia-G. Melis, Per una storia della bonifica della Nurra. Le carte Ascione (1918-1948), in A. Mattone-P. Sanna (a cura di), Alghero, la Catalogna, Il Mediterraneo cit., pp. 635-39. effettuata e la sua messa a disposizione di terzi, sia in forma gratuita sia a pagamento. 
L'Efc iniziò i lavori nel 1933 su di un'area di circa 30.000 ettari, che comprendeva sia i terreni di una colonia penale che una zona completamente improduttiva e malarica del demanio comunale, salvaguardando così gli interessi dei grandi proprietari terrieri. Il metodo con cui procedettero i lavori era innovativo: invece di aspettare che l'area fosse totalmente bonificata, si avviò il trasferimento dei coloni mentre i lavori erano ancora in corso, con la conseguenza che molti furono infettati dalla malaria o ebbero grosse difficoltà a rendere produttivi i loro terreni. Ciononostante, per le dimensioni del territorio e per la rapidità con la quale procedettero i lavori, si trattava di un'opera imponente rispetto a quelle precedenti, e già nel 1935 furono assegnate 65 case coloniche e l'anno successivo altre 129 persone si trasferirono nell'area, trasformando una zona disabitata e malsana per secoli in area produttiva ed amena grazie al regime e alle capacità del «nuovo italiano» ${ }^{42}$. Al centro di quest'area, inoltre, venne costruito un aeroporto militare, fondamentale per controllare i collegamenti con le Baleari e la penisola iberica durante la guerra civile spagnola, e che acquisì ancora più importanza durante la seconda guerra mondiale. Con queste opere il paesaggio algherese cambiò radicalmente, come rilevò il geografo Maurice Le Lannou che lo definiva «curioso, nuovo e morto allo stesso tempo, $[. .$.$] dove si può indovinare la natura refrat-$ taria e l'arrivata troppo rapida degli uomini» 43 .

La bonifica, comunque, era estranea alle necessità di crescita urbanistica e di miglioramento delle prospettive economiche della popolazione locale, e a tale proposito dobbiamo ricordare come ancora oggi sia diffusa l'idea che gli algheresi si siano autoesclusi in nome del loro atavico rifiuto a lavorare o abitare su quelle terre. Questo sembra il risultato della propaganda fascista, perché malgrado malarica, la zona non era completamente inutilizzata: nelle aree non paludose vi si raccoglieva il crine (palma nana), una fibra naturale che veniva lavorata in alcuni stabilimenti cittadini, e si praticavano la pastorizia e la caccia al cinghiale, due attività importanti nell'economia isolana. Inoltre, in base ai dati disponibili circa la manodopera disponibile, è evidente come esistesse un problema di occupazione che colpiva soprattutto braccianti agricoli e manovali ${ }^{44}$.

Del resto sia i coloni che il personale tecnico-amministrativo provenivano in prevalenza dalla provincia di Ferrara, una delle culle del fascismo, e l'esclusione dei locali lasciava presagire l'obiettivo di dar vita a una colonia di fascisti perfetti. Però, come già era avvenuto per l'Agro Pontino, la volontà di "utilizzare" un materiale umano di sicura fiducia si scontrò con l'esigenza delle autorità locali di eliminare gli elementi più irrequieti e problema-

42 E. Tognotti, L'economia e la società algherese tra le due guerre cit., p. 633; Archivio di Stato di Ferrara (d'ora in poi Asf), Archivio di Prefettura, Riservato di Gabinetto, b. 97, fasc. III, ins. C, c. 8 .

${ }_{43}$ M. Le Lannou, Pastori e contadini di Sardegna (1979), Edizioni della Torre, Cagliari 2006, p. 375 (ed. or. Tours 1941).

44 Asca, b. 927, fasc. 18 , cc. 5-6. 
tici. Almeno per quanto riguarda i coloni, alcuni furono scelti tra quanti erano considerati "indesiderabili" (oppositori, piccoli criminali, vagabondi ma anche fascisti troppo radicali) ${ }^{45}$; e così l'Efc dovette espellerne alcuni, sia perché si erano dimostrati poco adatti al lavoro agricolo, sia perché considerati «di idee contrarie al regime» 46 .

L'Ente di bonifica aveva creato una struttura sociale fortemente gerarchizzata dove i coloni, aldilà della loro effettiva fedeltà politica, erano completamente sottoposti al controllo dei suoi funzionari e, naturalmente, della milizia, come si evince dai «bilanci morali» che venivano inviati al Consiglio di Amministrazione dell'Ente o al Capo del Governo e che contenevano, oltre ad informazioni tecniche, molti particolari sulle famiglie coloniche ${ }^{47}$. Nella maggior parte dei casi, poi, impiegati, tecnici e amministratori dell'Efc erano fascisti di vecchia data che avevano anche preso parte alle violenze dei primi anni '20, rendendo questa istituzione una delle più fascistizzate. Il controllo era pressoché totale, e l'Ente, dopo aver bonificato il terreno ed aver costruito gli stabili, consegnava un «podere» ad ogni famiglia, la quale ne acquisiva la proprietà dopo 15 o 30 anni, nel corso dei quali doveva attenersi a varie regole e, soprattutto, pagare il debito contratto nell'accettare il terreno. In caso contrario - e anche in caso di mancanza di un'istruzione tecnica adeguata - era previsto il rimpatrio.

La comunità ideale fascista costruita nel comprensorio di bonifica, inoltre, comportò l'arrivo di un certo numero di persone provenienti dalla penisola in quella che potrebbe essere vista come una risposta alla prima colonizzazione catalana, avvenuta nel XIV secolo. Il nuovo nucleo di popolazione, destinato a crescere con il progredire dei lavori, rappresentava un'importante rottura rispetto al passato, perché introduceva una comunità completamente estranea, da un punto di vista linguistico, in una realtà sotto questo aspetto ancora molto compatta e scarsamente italianizzata. Si pensi soltanto che uno degli elementi che avevano garantito la sopravvivenza del catalano di Alghero era la necessità, per i nuovi arrivati, di apprendere tale idioma. A questo aspetto italianizzante si può aggiungere come i lavori di bonifica portarono ad una fascistizzazione della società algherese, tramite il trasferimento di molti fascisti radicali in un territorio nel quale questi erano praticamente assenti. In effetti, analizzando i dati sul tesseramento del fascio di Alghero, è facile notare come coloro che vantavano il titolo di «squadrista» non erano originari di Alghero, ma erano per lo più ferraresi, come funzionari dell' $\mathrm{Efc}^{48}$.

La colonizzazione interna, dunque, piuttosto che rispondere a problemi occupazionali ed economici, era un importante elemento nella costruzione di una società fascista basata sulla gerarchia e sulla condivisione dei valori della

${ }^{45}$ R. Mariani, Fascismo e "città nuove" cit., p. 161.

${ }^{46}$ Asf, Archivio di prefettura, riservato di Gabinetto, b. 97, fasc. III, ins. C, cc. 3, 6. Si tratta di alcuni esempi di provvedimenti di rimpatrio.

${ }^{47}$ Ivi, cc. 4, 9, Alghero, 18 agosto 1936.

48 Asca, b. 1178, f. 5 , cc. 40 e 41. 
«stirpe italica», che il regime, nelle sue aspirazioni totalitarie ed imperiali, cercava di realizzare. L'Enciclopedia italiana, alla voce «bonifica», se da una parte affermava che le ragioni di queste opere risiedevano nella «importanza data alla conservazione nella compagine nazionale di un alto grado di ruralità, e l'autarchia economica», dall'altro precisava come entrambi gli obiettivi fossero perseguiti solo in quanto necessari a realizzare l'utopia fascista ${ }^{49}$. Se consideriamo poi i legami culturali e linguistici della cittadina con il mondo catalanoparlante, ecco che il caso della bonifica nel territorio di Alghero acquista un aspetto di italianizzazione che va oltre la dimensione igienica (lotta alla malaria) e tecnico-economica (trasformazione fondiaria) nelle quali è sempre stata inquadrata, anche perché l'ambizioso progetto prevedeva la costruzione di un nucleo urbano, Fertilia, che costituitosi in comune avrebbe amputato il territorio storico del Comune di Alghero.

\section{La città nuova}

La progettazione del nuovo centro fu affidata inizialmente ad Arturo Miraglia, un ingegnere napoletano, fascista della prima ora e legionario a Fiume che aveva trovato una sistemazione economica e professionale nell'Efc (Miraglia fu responsabile di altre strutture realizzate nel territorio comunale) ${ }^{50}$. Egli approntò una planimetria generale con solo i progetti della chiesa e della scuola elementare (unico edificio realizzato di quelli da lui curati), dalla quale si intuiscono le ambizioni riposte nella «città nuova», che riassume tutte le caratteristiche e le contraddizioni dell'architettura di questo genere. Infatti, se la pianta radiale, la scuola e la distribuzione delle principali arterie stradali sono di ispirazione futurista, al contrario le dimensioni degli edifici e la presenza di abbondanti spazi verdi ricordano il modello della «città giardino» teorizzato da Ebenezer Howard ${ }^{51}$, ed esprimono quella dimensione rurale dentro la città che il fascismo aveva posto a garanzia di italianità e di fertilità. Anche Fertilia era organizzata attorno ad uno spazio monumentale dove radunare la popolazione durante le grandi manifestazioni e collocare gli edifici amministrativi dello Stato e del partito: una piazza imponente che si estendeva dal sagrato della chiesa fino ad una terrazza sul mare, simile alla poppa di una nave. In questa planimetria risaltano soprattutto le dimensioni della piazza e dello stadio che, spropositate anche rispetto all'attuale popolazione, erano state pensate per accogliere grandi masse di coloni ${ }^{52}$.

${ }^{49}$ A. Serpieri, Bonifica, Enciclopedia italiana di scienze, lettere ed arti, appendice II, Istituto dell'Enciclopedia Italiana, Roma 1936, pp. 295-301.

50 E. Cocco, Fertilia, Novecento, Albano Laziale 2007, pp. 59 ss.

${ }^{51}$ R. Fisherman, Urban utopias in the twentieth century, Mit, Cambridge 1982, pp. 21-88; E. Howard, Garden Cities of To-morow (1898), S. Sonnenschein \& Co., London 1902 (trad. it. Calderini, Bologna 1972).

52 E. Cocco, Fertilia cit., pp. 13-17. 
Questo progetto venne poi rielaborato nel 1937 dal gruppo di architetti che aveva già lavorato su Aprilia, il cosiddetto gruppo 2Pst, che prendeva il nome dalle iniziali dei suoi membri: Emanuele Filiberto Paolini, Concezio Petrucci, Riccardo Silenzi e Mario Tufaroli Luciano. Questi applicarono grosso modo le stesse soluzioni già sperimentate nell'Agro Pontino, e rispetto al disegno originale ridussero le dimensioni della piazza monumentale e aggiunsero un quartiere per ospitare il personale dell'aeroporto militare che, nel frattempo, l'Efc si era impegnato a costruire. Il cambiamento sostanziale fu nello stile architettonico, che lasciò da parte l'ispirazione futurista per favorire sia il «novecento» che il razionalismo italiano, entrambi reinterpretazione in chiave fascista e moderna della tradizione italiana ${ }^{53}$. Il risultato fu una cittadina che riassume, in pochi metri quadrati, tre momenti della storia dell'architettura italiana della prima metà del secolo scorso.

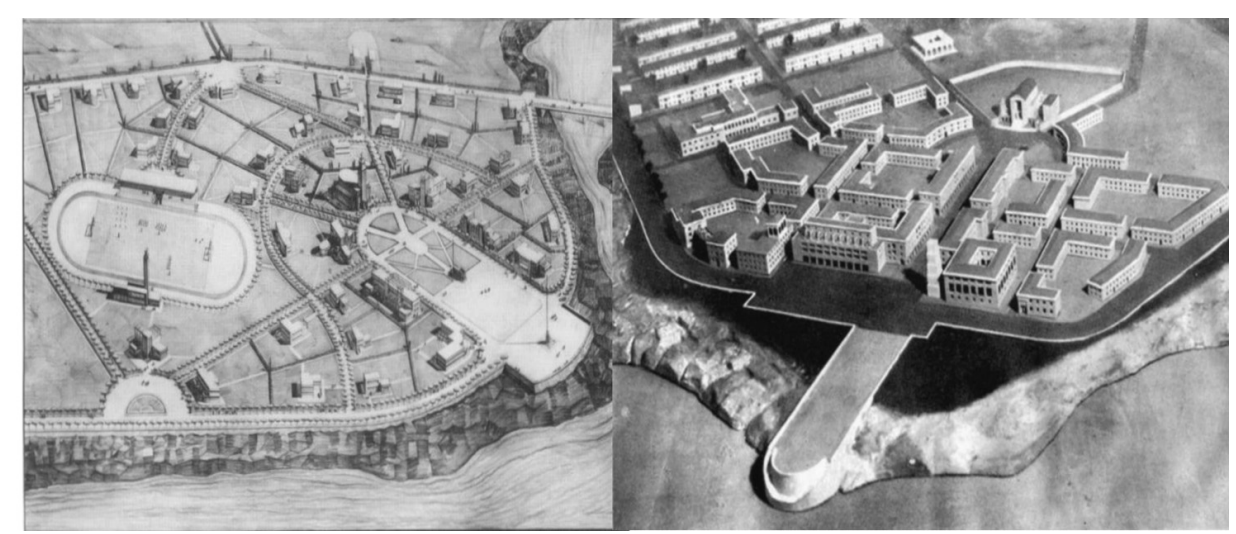

Assonometria di Fertilia dell'ing. Miraglia e plastico del progetto realizzato dal gruppo 2Pst. È possibile notare le caratteristiche tecniche della città, nonché l'evoluzione da un progetto avanguardista a uno più legato alla tradizione italiana. Fonte: E. Cocco, Fertilia cit., pp. 11 e 36

Fertilia fu inaugurata l'8 marzo del 1936 quando in realtà l'unico edificio completato era la scuola elementare. La cerimonia fu un atto spudoratamente propagandistico, dato che la città era ancora in costruzione, e vi parteciparono il presidente dell'Efc Mario Ascione assieme ad altre personalità dell'establishment fascista locale e nazionale. La retorica gravitava attorno ai miti della redenzione della terra e della «rinascita» che il fascismo aveva promesso per l'isola, collegando il centro alla civiltà che avanzava, in Sardegna come in Etiopia. Infatti, durante la posa della prima pietra della chiesa e della

53 Ivi, pp. 37-41; A. Cucciola, Vecchie città/città nuove. Concezio Petrucci, Dedalo, Bari 2006, pp. 257-63; G. Peghin, Fertilia. 1935-1937, «Parametro», 2001, n. 235, pp. 32-37. 
casa del fascio, si inserirono nelle fondamenta due pergamene di Filippo Figari, uno dei migliori interpreti degli ideali sardisti nelle arti figurative che, aderendo al regime, continuò a lavorare sulla sardità, però in chiave fascista ${ }^{54}$. In quella interrata sotto la casa del fascio, che simbolicamente rappresentava il cuore della nuova Italia, si poteva leggere:

Il dì 8 marzo 1936, XIV dell'Era Fascista, mentre l'Italia fieramente reagendo alle sanzioni strappa alla sua terra recondite ricchezze e doma inflessibile la barbarie etiopica, qui a Fertilia città primogenita dell'Ente Ferrarese, all'umano lavoro redenta regnando Vittorio Emanuele III si pone la prima pietra della casa del fascio littorio, simbolo dell'anima Romana, da Benito Mussolini virilmente rinnovata nell' italica stirpe ${ }^{55}$.

È evidente il legame con la costruzione dell'Impero, tanto che quando questo fu ufficialmente proclamato, la piazza principale di tutte le «città nuove» fu intitolata a questa data storica (9 maggio). Nella liturgia fascista, infatti, esisteva un parallelo tra la civiltà che avanzava in Etiopia e quella che veniva avanti nella Nurra, tra la colonizzazione di spazi incolti o poco antropizzati dentro il territorio nazionale e quella dell'Africa: si trattava della prova, ad uso propagandistico sia interno che esterno, del successo del regime nel realizzare i suoi obiettivi e nel "portare la civiltà». Questo aspetto, che è stato messo in luce a partire dell'analisi della bonifica delle Paludi Pontine ${ }^{56}$, si presenta secondo gli stessi parametri nel caso di Fertilia. Anche qui i cinegiornali ed i documentari giocano sul binomio barbarie/civiltà, dove la prima è rappresentata dalla natura incolta ed aspra della Nurra, dalla pastorizia seminomade e dalle misere abitazioni di pietra e paglia usate dai pastori; al contrario la civiltà è espressa prima dal lavoro di bonifica, descritto spesso come un'impresa bellica, e infine dal paesaggio addomesticato, trasformato in produttivo e civilizzato. Un altro elemento che nei filmati dell'epoca raffigurava questa opposizione è la figura del colono ferrarese che, a differenza del pastore sardo intento "solo" a guardare il gregge, è ritratto come una persona molto attiva e sempre impegnata in qualche lavoro agricolo (al contrario il pastore è sempre ripreso mentre siede su una pietra $)^{57}$. Se poi consideriamo che le capanne in cui vivevano questi pastori erano simili a quei tucul etiopi divenuti simbolo per eccellenza dell'inciviltà africana, è chiaro come Fertilia

54 S. Paulis, La costruzione dell'identità. Per una analisi antropologica della narrativa in Sardegna fra''800 e '900, Edes, Cagliari 2006, pp. 388-89.

55 F. Figari, Pergamena per l'inaugurazione della casa del Fascio di Fertilia, 1936, ora in E. Valsecchi, Anni di pace e anni di guerra. Fertilia, «Nuova Comunità», n.s., (1997), p. 67.

56 F. Caprotti, Mussolini's Cities cit., pp. 219-45.

57 Sardegna Digital Library, Documentario Incom, 1940 Ca., in www.sardegnadigitallibrary.it/index.php?xsl=626\&s=17\&v=9\&c=4460\&id=225872; Archivio Storico dell'Istituto Luce, Giornale Luce B0853, in www.archivioluce.com/archivio/jsp/schede/videoPlayer.jsp? tipologia $=\& \mathrm{id}=\&$ physDoc $=11723 \& \mathrm{db}=$ cinematograficoCINEGIORNALI\&findIt=false \&section $=$. 
e le altre "città nuove" "erano presentate dal regime come le avanguardie della civiltà fascista» ${ }^{58}$ e avevano un notevole valore simbolico.

Nonostante questa importanza i lavori procedettero lentamente, a causa soprattutto della guerra, e quando questa finì Fertilia sembrava un quadro di De Chirico, con una piazza e qualche strada abbozzata, una chiesa in costruzione accanto ad una scuola futurista e verso il mare le due torri, quella comunale e quella del fascio, che si ergevano dal nulla. Anche se l'idea fascista di Fertilia non fu realizzata del tutto, è importante analizzare il significato di questa nuova città e della bonifica in relazione ad Alghero. Infatti a partire dall'analisi della funzionalità del nuovo centro, delle politiche demografiche e di gestione della popolazione, nonché del simbolismo implicito nell'architettura, si può concludere che Fertilia fu pensata come alter ego fascista ed italiano di Alghero, un centro ancora caratterizzato dall'architettura gotica e da una tradizione culturale catalana.

Come abbiamo visto la logica che portò alla colonizzazione non rispondeva affatto alle esigenze concrete del territorio di Alghero. A Fertilia e nei terreni bonificati avrebbero dovuto vivere esclusivamente coloni provenienti dalla provincia di Ferrara e militari della Regia Aeronautica, mentre l'unica ricaduta positiva sul territorio era nel settore della costruzione, dato che le ditte appaltatrici erano di Alghero e Sassari ${ }^{59}$. Ma a parte un momentaneo beneficio sulla disoccupazione, la trasformazione della Nurra appariva completamente estranea agli occhi degli abitanti del luogo, tanto che non sembra si siano registrati episodi di conflitto con i coloni, a differenza di quanto avvenne nell'Agro Pontino. Che lo scopo principale non fosse il miglioramento delle condizioni di vita degli abitanti di Alghero, ma la creazione di un vivaio di italiani, è dimostrato anche dai criteri con cui si selezionavano i coloni, che oltre ad essere di origine contadina dovevano avere una certo numero di figli (anche se poi, come visto, questi criteri non sempre erano rispettati). A partire dal 1935, dunque, famiglie con una media di 6-10 figli furono trasferite nei nuovi poderi, tutti delle stesse dimensioni e tutti con lo stesso tipo di casa, dipinta di bianco e decorata con una fascia azzurra (il colore degli ex combattenti) e frasi sulla patria, sulle virtù della vita rurale e sull'importanza della prolificità, tra le quali la più emblematica era: «se le culle sono vuote, la nazione invecchia e decade» ${ }^{60}$.

È chiaro, dunque, come Fertilia e la bonifica della Nurra rispondessero ad una logica di miglioramento della stirpe e di inversione del declino demografico, tanto che la scuola e le strutture sanitarie progettate avevano delle caratteristiche singolarmente d'avanguardia. Malgrado il nuovo centro distasse solo $6 \mathrm{~km}$ da Alghero, si pensò di realizzare un ospedale e una rete di ambula-

${ }^{58}$ F. Caprotti-M. Kaika, Producing the ideal fascist landscape cit., p. 619.

${ }_{59} \mathrm{Nel}$ febbraio del 1935, secondo un documento dell'Efc, le persone disponibili per questi lavori erano più di 500, cfr.: Asf, Archivio di prefettura, Riservato di Gabinetto, b. 97, fasc. III, ins. C, c. 4.

60 Testimonianza di A.F. (1930-), cfr. nota 41. 
tori sparsi per il comprensorio di bonifica che, oltre a garantire l'assistenza medica, sembrano essere stati progettati perché i futuri lavoratori e soldati, ma anche le future madri e mogli, fossero costantemente monitorati per garantirne una sana e robusta costituzione fisica. Anche la struttura della scuola elementare rispondeva agli stessi criteri e, a parte l'interesse per uno dei pochi esempi di architettura futurista nell'isola, le soluzioni adottate dimostrano la volontà di assicurare la crescita di italiani e fascisti in perfette condizioni fisiche e morali. L'edificio, infatti, era formato da due aule principali a forma semicircolare e di grandi dimensioni, con delle enormi finestre che garantivano l'illuminazione naturale, mentre un sistema di prese d'aria e condotti favoriva la ventilazione. Nella scuola, inoltre, erano presenti una mensa, alcuni appartamenti per ospitare gli insegnanti con tanto di balcone per i discorsi agli alunni, e un ambulatorio. In questo modo i figli dei nuovi arrivati sarebbero stati educati in un edificio d'avanguardia, con tre fasci littori che dominavano l'entrata principale e che erano ben visibili da ogni lato della struttura, immersa in quell'ambiente rurale considerato come la miglior garanzia per mantenere i valori della "stirpe". Inoltre la scuola, dedicata alla madre di Mussolini, fu il primo edificio al quale si iniziò a lavorare e fu l'unico terminato prima della caduta del regime: il che conferma quanto si scommettesse sulle future generazioni per creare quei «romani della modernità» dei quali parlava il regime (l'edificio, recentemente restaurato, è tuttora la sede della scuola elementare).

Se poi analizziamo la serie di rapporti simbolici che si stabiliscono tra l'architettura di Fertilia e quella di Alghero, risulta più chiaro il tipo di relazione che si intendeva instaurare tra i due centri. In questo senso il primo elemento da considerare è la collocazione nello spazio: mentre altre città come Aprilia o Pontinia furono realizzate al centro del comprensorio di bonifica, Fertilia si affaccia sul mare al limite sud dell'area interessata dai lavori e quindi è il suo sbocco naturale al mare. La «città nuova» fu edificata in un luogo conosciuto come Península del Gall (promontorio del Gallo, in catalano), esattamente speculare al promontorio dove sorge Alghero e del quale differisce solo per posizione e dimensioni. Infine la sua ubicazione nel punto in cui lo stagno del Calik si riversa in mare suggerisce come Fertilia non fosse destinata ad essere solo un centro agricolo, ma anche un porto di pescatori (attualmente vi sorge un porto turistico). Dunque, secondo questi elementi, il nuovo centro era stato pensato come un'alternativa ad Alghero, che tradizionalmente viveva della pesca e della lavorazione ed esportazione dei prodotti agricoli del suo territorio.

Data poi l'importanza conferita all'architettura quale strumento per poter plasmare la realtà alla maniera fascista, è poco probabile che questa posizione fosse stata scelta per motivi diversi da quelli - voluti - di una tensione spaziale, e simbolica, tra le due città ${ }^{1}$. La stessa sensazione dovette provarla

${ }^{61}$ Sull'importanza dell'architettura per il fascismo cfr. E. Gentile, Fascismo di pietra, Laterza, Roma-Bari 2007. 
un commentatore dell'epoca, Stanis Ruinas, un fascista sardo di tendenze radicali che durante quegli anni pubblicò un libro apologetico su Mussolini e le «città nuove» ${ }^{62}$. L'autore, che nell'introduzione chiariva come la colonizzazione interna serviva a «riscattare la terra, e con essa gli uomini, e con gli uomini la razza», comincia il suo itinerario proprio da Alghero, e descrive la città catalana e la Nurra con immagini di arcaicità che si scontrano duramente con il racconto della trasformazione fascista del territorio; questa opposizione tra due mondi differenti è evidente nella descrizione della prima visione dei lavori:

Proprio sull'orlo occidentale di questa plaga immensa [la Nurra], nell'arco del golfo algherese e in riva all'acqua stava sorgendo Fertilia, quasi a sfatare col suo stesso nome la leggenda della terra stregata e l'occulto potere chiuso nel nome del vigilante monte Doglia. Ne ebbi la prima visione dall'arco della porta che attraverso una breve galleria sbocca sul porticciolo della Piazza Civica di Alghero [...]. In quel cielo quasi glauco, attraverso i barcozzi variopinti e le chiatte tirate a secco fin dentro il giardino pubblico, ebbi l'impressione che ogni limite fra l'acqua e la terra fosse scomparso e tutte quelle case bianche, lontane, che punteggiavano la tela del mio pastello incorniciato di pietra, fossero vele immobili nella calma dei venti ${ }^{63}$.

L'opposizione spaziale è perfetta, ed effettivamente l'asse centrale della piazza monumentale della nuova città è allineato con il campanile della cattedrale gotico-catalana di Alghero (Santa Maria) ${ }^{64}$. Quasi sembra che gli architetti abbiano voluto rendere ben visibile Fertilia a quanti si trovassero al porto o sui bastioni della città antica, come se volessero dare agli algheresi una prova concreta che il regime stava costruendo un mondo nuovo.

Un altro elemento è importante per sottolineare questa contrapposizione voluta tra i due agglomerati: il contrasto architettonico. Fertilia rappresentava l'urbanesimo moderno, con la sua scuola futurista, gli ampi spazi, le strutture funzionali, le strade pensate per essere attraversate da automezzi ed addirittura gli edifici disposti secondo l'asse eliotermico per sfruttare al massimo l'illuminazione ed il calore del sole. Al contrario, Alghero era una cittadella medievale, con un centro storico sovrappopolato e con edifici troppo alti per permettere al sole di penetrare nelle strade, strette e tortuose tanto da non poter essere percorse facilmente da automobili; non disponeva né di piazze né di spazi monumentali (le piazze che si possono ammirare oggi sono il risultato della ricostruzione dopo i bombardamenti alleati del 1943). Nella città fascista, poi, dove l'uso scenografico dell'architettura era molto importante, lo spazio era dominato dalle torri della Casa del fascio e del Comune, mentre era completamente assente il campanile (fu realizzato solo nel 1955), segno che la religione fascista, nelle utopie del regime, prevaleva di gran lunga su

${ }^{62}$ S. Ruinas, Viaggio per le città di Mussolini, Bompiani, Vicenza 1938. Su Ruinas, cfr. P. Buchignani, Fascisti rossi, Mondadori, Milano 1998.

${ }^{63}$ S. Ruinas, Viaggio per le città di Mussolini cit., p. 17.

${ }^{64}$ E. Cocco, Fertilia cit., p. 14. 
quella cattolica; al contrario l'elemento che spicca nella città medievale di Alghero erano i due campanili gotici, visibili da ogni prospettiva. In poche parole, Alghero rappresentava quell'architettura urbana che il regime attribuiva all'Italia prefascista, e che, come tutto ciò che si poteva riferire a quel periodo storico, Mussolini si era proposto di eliminare o trasformare. Al lato opposto del suo golfo, quindi, si progettò la costruzione di un suo alter ego architettonico, una cittadina, per usare le stesse parole dei progettisti, dalla «fisionomia spiccatamente italiana e mediterranea» 65 .

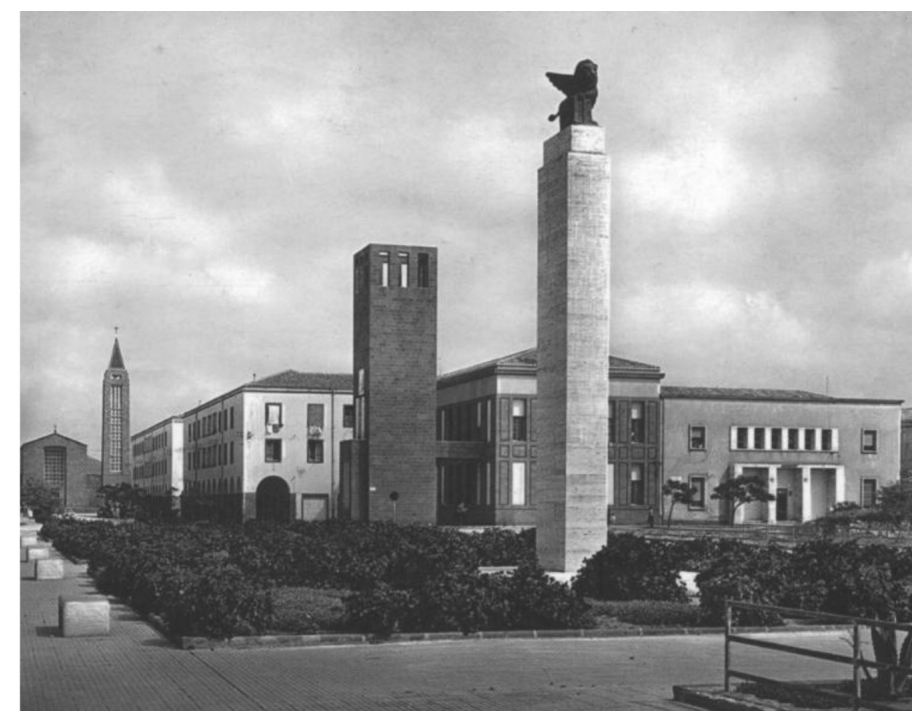

Veduta di Fertilia dalla piazza monumentale. Il leone di S. Marco è stato aggiunto dai profughi giuliano-dalmati arrivati nel secondo dopoguerra, ed è oggi il simbolo della città. Fonte: cartolina postale anni '50

Ma l'elemento che maggiormente rende l'idea di come le due città fossero antagoniste è dato dal fatto che Alghero costituiva, allora, una comunità catalanoparlante molto compatta che, malgrado i discendenti dei coloni catalani fossero pochissimi, manteneva ancora la sua peculiarità linguistico-culturale. Chi vi immigrava era costretto, per potersi integrare, ad apprenderne l'idioma, tanto che la locale produzione letteraria in catalano, allora come oggi, è da attribuirsi in buona parte ad immigrati di seconda o terza generazione. Inoltre ad Alghero a cavallo tra il XIX ed il XX secolo si era sviluppato un movimento letterario di stampo catalanista, con alcuni dei suoi rappresentanti

65 Ivi, p. 42. 
che si dichiaravano favorevoli alla "nazione" catalana, mentre qui il movimento sardista aveva opposto una certa resistenza al fascismo 66 . Questi elementi erano sufficienti per giustificare una politica di italianizzazione e di fascistizzazione che, in parte, si basava sull'instaurazione di una colonia di ferraresi e la costruzione di una città antagonista. In altre parole questa realtà, malgrado per i fascisti non fosse tanto pericolosa come la minoranza tedesca dell'Alto Adige o quella slovena di Trieste, non coincideva affatto con quanto affermava Mussolini alla Camera dei fasci e delle corporazioni proprio a un anno dall'entrata dell'Italia nella seconda guerra mondiale: «gli stati devono aspirare a realizzare il massimo della propria unità etnica e spirituale, per poter far coincidere ad un certo momento i tre elementi di razza, nazione e stato» 67 .

In conclusione Fertilia e la bonifica della Nurra, piuttosto che soddisfare le esigenze di sviluppo economico locale ed essere parte di quel programma di rinascita regionale che il regime aveva prospettato ai sardisti nel momento della fusione tra i due movimenti, sembrano rispondere a molteplici ragioni. Infatti, come scrive Federico Caprotti nella sua monografia sulla bonifica dell'Agro Pontino, la realizzazione di queste città era sempre legata a diverse motivazioni, quasi mai ad una in particolare ${ }^{68}$. Fertilia, dunque, non era dovuta solo alla disoccupazione nell'area di Ferrara, allo spopolamento cronico della Nurra o all'arretratezza economica della Sardegna, secondo l'opinione più diffusa in ambito locale ${ }^{69}$. La colonizzazione fascista serviva piuttosto al bisogno di attuare politiche di incremento (quantitativo e qualitativo) demografico, ed era uno degli strumenti per realizzare definitivamente l'italianizzazione di un territorio che, per una serie di ragioni storiche e sociali, non era stato perfettamente integrato nella nazione. Fertilia era la nuova comunità, fascista e italiana, destinata a neutralizzare la vecchia, dalle caratteristiche alloglotte. A questo proposito può servire fare un breve paragone con un'altra «città nuova» realizzata quasi negli stessi anni nel Dodecaneso: Portolago

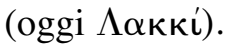

Nelle isole italiane dell'Egeo era in corso, fin dagli anni '20, una politica di progressiva assimilazione delle popolazioni autoctone, che durante il conflitto si spinse fino alla concessione di un particolare status di cittadinanza (la cosiddetta «piccola cittadinanza»). Dal punto di vista urbanistico gli interventi furono imponenti, a Rodi in particolare, dando alle isole una fisionomia architettonica che, recuperando elementi veneti e bizantini, ha contribuito notevolmente a forgiare l'odierna immagine turistica di queste località. In questo

${ }^{66}$ R. Caria, I retrobaments ad Alghero fra Otto e Novecento, in J. Carbonell-F. Manconi, I catalani in Sardegna cit., pp. 183-86; E. Tognotti, L'economia e la società algherese tra le due guerre (1919-1939), in A. Mattone-P. Sanna (a cura di), Alghero, la Catalogna, il Mediterraneo cit., p. 626.

67 D. Rodogno, Il nuovo ordine mediterraneo cit., p. 322.

${ }^{68}$ F. Caprotti, Mussolini's cities cit., pp. XX-XXVI.

${ }^{69}$ E. Valsecchi, Anni di pace, anni di guerra cit., pp. 41-49. 


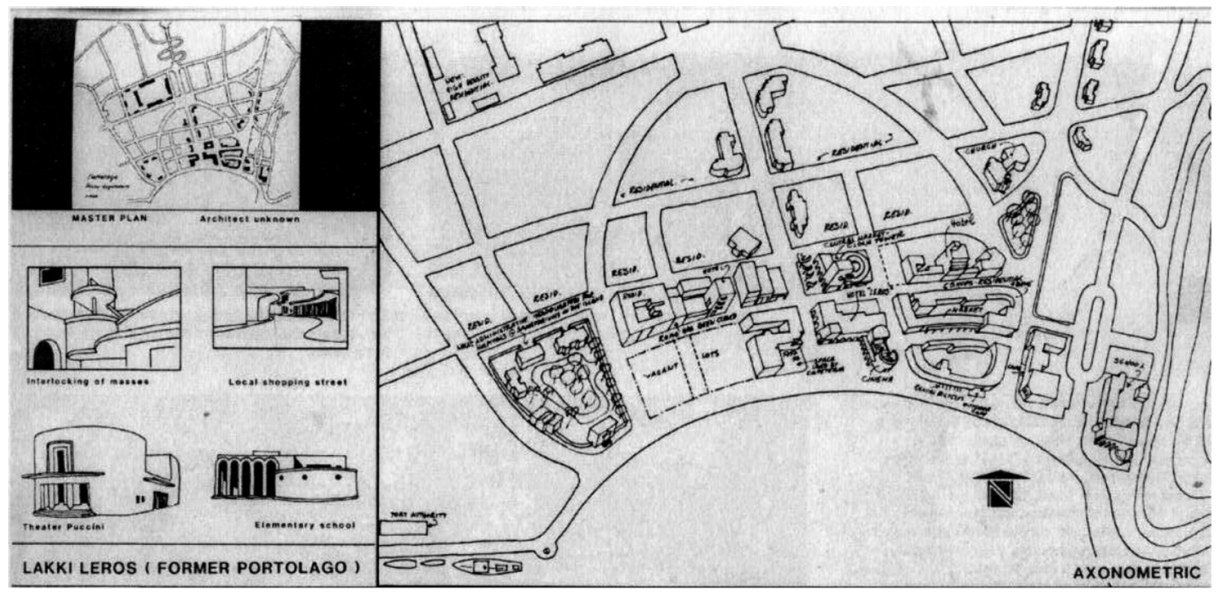

Planimetria di Portolago. Notare le somiglianze con Fertilia, soprattutto la forma quasi semicircolare del centro e la rete stradale. Fonte: A.C. Antoniades, Italian architecture in the Dodecanese: a preliminary assessment, «Journal of Architectural Education», 1984, n. 38, p. 23

quadro si deve inserire la costruzione di Portolago, realizzata tra il 1934 ed il 1936 nell'isola di Lero ( $\Lambda$ ท́ $\rho \circ$ s), davanti alle coste della Turchia, in quello che è il miglior approdo naturale del Mar Egeo e che durante gli anni '30 divenne la principale base aeronavale italiana nell'area. In quest'isola abitata da 4.000 persone, si progettò di trasferire 30.000 italiani, in buona parte militari con le rispettive famiglie ma anche, come nelle colonizzazioni interne, contadini ${ }^{70}$. Lo scopo della costruzione di questa cittadina andava ben oltre la soddisfazione delle necessità abitative dovute alla presenza di strutture militari, ma era finalizzata anche all'italianizzazione come alcuni elementi, oltre l'ingente numero di italiani che vi si trasferirono, suggeriscono. Innanzitutto Portolago, al contrario di quanto era avvenuto fino ad allora nel Dodecaneso con la rielaborazione del passato coloniale veneto da parte degli architetti italiani, fu realizzata secondo i canoni del razionalismo fascista (o italiano) e risulta molto interessante proprio perché, assieme a Sabaudia, è l'unica città interamente progettata secondo questo stile. Quindi, invece di cercare un'integrazione degli autoctoni attraverso la rielaborazione del passato, qui si adotta una soluzione più radicale, provando a contrapporre al nucleo storico di abitanti una comunità italiana e fascista. Inoltre l'insediamento era anche il centro nevralgico di una serie di aziende agricole che, significativamente, erano dedicate agli italiani, mentre gli isolani, come nel caso di Fertilia, fornivano unicamente la manodopera per il settore della costruzione e non pote-

70 Leros: Little Italy, «The Independent», 9 settembre 2006, www.independent.co.uk/travel/ europe/leros-little-italy-415168.html. 
vano risiedere nel nuovo agglomerato urbano ${ }^{71}$. Naturalmente in questo caso si trattava di un territorio conquistato solo pochi anni prima durante la guerra italo-turca (1911-1912), e pertanto era oggetto di una particolare politica di italianizzazione. Questa si basava sulla possibilità o meno di assimilare la popolazione locale: dopo aver stabilito che gli autoctoni avevano un soddisfacente grado di «pertinenza», ovvero di somiglianza alla razza italiana, si procedeva ad un'opera di italianizzazione e fascistizzazione che era affidata al partito, all'educazione, all'uso della simbologia del regime e, in casi estremi, alla colonizzazione o addirittura all'espulsione della popolazione locale.

La caduta del regime fascista ha determinato la fine di questi progetti, lasciando Fertilia come un grosso cantiere in costruzione e obbligando i coloni italiani che si erano insediati a Portolago ad abbandonare l'isola, oggi territorio greco. Fertilia divenne paradossalmente non una comunità ideale, così come i fascisti la immaginavano, ma un porto di salvezza per i profughi italiani provenienti dall' Adriatico, che in fuga dall'avanzata dei partigiani titini e dalla loro violenta risposta alla pulizia etnica praticata dai fascisti durante l'occupazione dell'Istria e della Dalmazia, finirono per stabilirsi anche sulle coste sarde $^{72}$. Così non una comunità di ferraresi, ma profughi istriani e dalmati terminarono Fertilia. Questi costituiscono tuttora la maggioranza dei suoi abitanti, facendo del centro un enclave in un territorio che era già stato in passato un enclave catalano. I toponimi di Fertilia sono tutti riferiti ai luoghi d'origine dei profughi (via Zara, via Pola ecc.), gli abitanti non parlano il catalano di Alghero, ma hanno conservato le loro parlate locali, mentre partecipano difficilmente alle festività o ritualità proprie della tradizione storica del centro vicino. Significativamente, a Fertilia non si festeggia il 25 aprile come festa della Liberazione, che al contrario è sentita come una tragedia, ma si celebra in quella data S. Marco, patrono della cittadina e legame simbolico con il passato veneto, e poi italiano, delle terre abbandonate dai profughi. Nei terreni dell'Efc, che cambiò nome più volte ma che sostanzialmente continuò a svolgere le stesse funzioni, non si insediarono solo i coloni ferraresi o i profughi: la maggioranza delle terre furono occupate da contadini sardi nel primo dopoguerra, mentre altre furono bonificate nei decenni successivi ${ }^{73}$.

Oggi, sebbene tra gli abitanti dei due centri si siano instaurati discreti rapporti di vicinato, Fertilia si è trasformata proprio in quella nuova comunità destinata a contendere il territorio alla vecchia comunità. In effetti oggi si può affermare come la colonizzazione iniziata in epoca fascista, con l'introduzione di nuclei di popolazione che non conoscevano e non erano obbligati

\footnotetext{
${ }^{71}$ A.C. Antoniades, Italian architecture in the Dodecanese cit.; V. Colonas, Italian architecture in the Dodecanese Islands (1912-1943), Oloko, Atene 2002, pp. 66-71; D. Pizzi, Città metafisiche cit., pp. 89-93.

72 M. Molinari, L'emigrazione dei profughi giuliani in Sardegna e Oltreoceano, «Storia e Futuro», 2010, n. 23, pp. 2-46.

73 M. Cardia, Le lotte contadine per la riforma agraria nel comprensorio di Alghero (19441950), in A. Mattone-P. Sanna, Alghero, la Catalogna, il Mediterraneo cit., pp. 643-67.
} 
a conoscere il catalano di Alghero, abbia infranto l'unità linguistica e culturale di questo territorio, ed abbia contribuito, assieme ad altri elementi come l'istruzione pubblica e la diffusione dei mezzi di comunicazione di massa, al progressivo abbandono dell'idioma locale da parte degli algheresi e, quindi, alla graduale perdita di quelle peculiarità storico-linguistiche che avevano reso la città un'isola dentro l'isola. 


\title{
Ricerca storica e cittadinanza attiva: intervista a Eric Foner
}

\author{
Daria Frezza e Alessandra Lorini
}

\begin{abstract}
Historical research and active citizenship: interviewing Eric Foner focuses on the interconnections between historical scholarship and a historian's active role as a citizen in the cultural and political events of American society. Beginning with his personal and cultural background Foner highlights the crucial points of his major works by placing them in the larger context of American historiography. He also suggests how the most recent results of his research can be brought to the attention of a larger public, well beyond the boundaries of academic circles.
\end{abstract}

Key words: history, citizenship, slavery, freedom, Reconstruction Parole chiave: storia, cittadinanza, schiavitù, libertà, Ricostruzione

Frezza-Lorini. In questa intervista ${ }^{1}$ affronteremo la questione del rapporto tra la tua ricerca storica e la tua partecipazione alle questioni della cittadinanza democratica. Ci interessa in particolare la tua ricerca relativa all'uso pubblico della storia. Affermi che la storia appartiene a tutti e a nessuno. Allora, cosa significa insegnare storia oggi? Come puoi convincere $i$ tuoi studenti che la storia oggi è di vitale importanza? Pensiamo che cercare di coinvolgere gli studenti a riflettere in modo critico sui temi trattati nelle lezioni sia fondamentale. Quale importanza ha lo studio della storia oggi quando in tutto il mondo i diritti umani e la cittadinanza democratica sono messi in discussione? Come possono legarsi tra loro il tuo senso etico della cittadinanza e la tua ricerca storica? Potremmo partire dal contesto nel quale ti sei formato, dal rapporto con tuo padre Jack e tuo zio Phil entrambi storici, così come lo affronti nel volume Who Owns History².

${ }^{1}$ Eric Foner è De Witt Clinton Professor of History alla Columbia University. Si è occupato di storia delle idee politiche, di storia sociale e dei rapporti razziali in America. Questa intervista si è svolta il 17 aprile 2012 a New York.

2 E. Foner, Who Owns History? Rethinking the Past in a Changing World, Hill and Wang, New York 2002. Si tratta di un volume di saggi in cui emergono gli elementi più rilevanti della biografia intellettuale dell'autore e riferimenti agli storici della sua famiglia quali il padre

«Passato e presente», a. XXXI (2013), n. 88 effettuata e la sua messa a disposizione di terzi, sia in forma gratuita sia a pagamento. 
Foner. Negli Stati Uniti abbiamo un atteggiamento per certi versi paradossale nei confronti della storia. Siamo orgogliosi di essere proiettati verso il futuro e non verso il passato. Thomas Paine affermò che avevamo il potere di far ripartire il mondo da capo. La storia era un peso che dovevamo toglierci di dosso e non era rilevante per l'America: apparteneva all'Europa, al Vecchio Mondo e l'idea stessa di America è di andare oltre la Storia. Ma la storia è importante ed è sempre con noi. Negli Stati Uniti c'è un grande interesse per la storia in generale. Il canale televisivo History Channell è molto popolare, i visitatori di musei storici, monumenti, siti e parchi nazionali di interesse storico sono molto numerosi. Nella lista dei bestseller sono sempre stati presenti libri di storia, anche se si tratta per lo più di biografie dei padri fondatori di livello scientifico non alto. Esiste dunque un pubblico per la storia anche fuori dal mondo accademico. Perciò ritengo che l'impegno di storici di professione come me sia quello di far leva su questo interesse per tentare di renderlo più ricco e più complesso. Gli studenti arrivano all'università con qualche conoscenza storica, ma con assai scarsa consapevolezza di ciò che lo studio della storia comporta, e hanno difficoltà a capire il concetto di interpretazione storica, come pure il fatto che la storia non è soltanto un insieme di eventi ma è un atto di immaginazione che crea un ordine tra loro in linea con una determinata interpretazione. Per esempio la Columbia University, dove insegno, ha una lunga tradizione di celebri docenti tra i quali Allan Nevins, Henry Steele Commager, Richard Hofstadter e oggi Alan Brinkley e Mark Mazower, storici molto conosciuti che attraggono un gran numero di studenti. Per questo non credo che il problema sia quello di trovare un pubblico interessato alla storia quanto piuttosto quello di indurre gli studenti a riflettere su cosa questa realmente sia, su cosa lo studio del passato comporti: si tratta di un continuo processo di costruzione di narrazioni e interpretazioni così da fornire strumenti per comprendere perché le interpretazioni della storia cambiano nel tempo. Credo che il problema sia quello di promuovere un'analisi critica e non solo una narrazione dei fatti: questo è ciò che ci proponiamo nel nostro insegnamento.

Lorini. Ricordo che quando ero una dottoranda insieme ai miei colleghi avevo la percezione che lavorare sotto la tua direzione era far parte di una comunità internazionale di giovani studiosi. Molti di noi, una volta finito il dottorato, sono tornati nei paesi di origine portando con sé il tuo insegnamento sull'importanza della storia e del rapporto tra ricerca storica rigorosa

Jack Foner, studioso del movimento operaio e del movimento dei diritti civili, autore, tra l'altro, di Blacks in the Military in American History (1974), e lo zio Philip Foner, storico marxista del movimento operaio e dei neri, autore prolifico e curatore di raccolte di documenti storici tra cui i 10 volumi di History of the Labor Movement in the United States (International Publishers, New York 1947-'94) e i 5 volumi di The Life and Writings of Frederick Douglass (International Publishers, New York 1950-'55 e suppl. del 1975). 
e cittadinanza responsabile. Puoi fare qualche esempio che spieghi l'importanza di questo rapporto?

Foner. I temi che mi hanno interessato come storico sono sempre stati legati alle problematiche del presente. I primi studi storici che intrapresi riguardavano i movimenti antischiavisti e questo avvenne negli anni '60 nel momento della rivoluzione dei diritti civili e del movimento dei neri; diversi storici - non solo io - iniziarono ad occuparsi di questi temi per capire da quali percorsi della nostra storia emergevano; molti studiosi si occuparono della schiavitù, dell'abolizionismo e del periodo della Guerra civile non solo per cercare nuove prospettive ma anche importanti intuizioni rispetto al presente, in modo da ricostruire le radici storiche della crisi che la società americana stava affrontando. Se guardate ai miei lavori in retrospettiva, vedrete che tutti hanno qualcosa da dire sul presente, sia che riguardino lo studio del periodo della Ricostruzione, il momento in cui tutta la questione dei diritti civili e il ruolo dei neri in America divenne per la prima volta un problema nazionale, sia che analizzino il ruolo di Tom Paine nella Rivoluzione americana, un libro che scaturì dal mio interesse per la storia del radicalismo americano negli anni '60. Oppure i miei lavori sulla libertà e la storia della libertà negli Stati Uniti: la libertà è un concetto assolutamente centrale nella cultura politica americana. Anche il mio libro più recente su Lincoln è centrato sostanzialmente sulla questione di come il cambiamento abbia luogo e sul rapporto tra leader politici e movimenti sociali. Quest'ultima è una domanda scaturita dal presente, poiché ho scritto questo libro durante la campagna di Obama. Questo non vuole affatto dire imporre il proprio punto di vista sul presente o il passato anche se sono in molti a farlo.

Frezza. Questo è un altro aspetto che vorrei tu affrontassi: la storia non deve essere appiattita sul presente, vero?

Foner. È il presente che pone allo storico le domande, ma non fornisce le risposte. Questo è ciò che costantemente cerco di dire ai miei studenti: le problematiche cui siete interessati nascono dalla vostra vita, dal vostro mondo. Poi però dovete seguire la direzione indicata dalla vostra ricerca d'archivio. Io ho cambiato molte volte le mie opinioni nel corso delle mie ricerche. Quella su Lincoln è cambiata, ma le domande non sono cambiate. Le risposte invece devono emergere dalla ricerca. Credo nel concetto di usable past, un concetto che mette in grado lo storico di pensare più efficacemente alle problematiche del presente, e che non produce risposte ma fornisce una specie di gerarchia in base alla quale pensare le questioni correnti: ed è qui che cittadinanza attiva e studio della storia si intersecano. Ma un usable past deve essere una buona storia, non una che si pensa servirà a qualche scopo del presente. Questo tipo di storia non è buona. Come può la gente di oggi apprendere realmente da una storia che non sia accurata e fondata su una ricerca solida? Sotto un certo profilo la rivoluzione storica iniziata negli anni ' 60 e '70 nello 
studio della storia americana emerse dal fatto che la storia precedente non era adeguata. Ciò che crollava era l'intero punto di vista del "consenso" nella storia americana, dominante negli anni ' 50 in cui sono cresciuto, secondo il quale non c'era mai stata alcuna seria divisione nella società americana e che tutti i problemi erano stati risolti. Secondo questo punto di vista si trattava soltanto di una questione di gestione della società: c'era una piccola scatola nella quale convivevano tutti i leader americani, al di fuori della quale non c'era niente, solo un pugno di fanatici e di maniaci da ignorare. Questo approccio storico come poteva spiegare il mondo in cui si viveva negli anni '60? Tutta la società cadeva a pezzi e gli storici non erano in grado di fornire una spiegazione all'altezza dei problemi del presente.

Frezza. A quel punto tu eri a Columbia e studiavi con Richard Hofstadter $^{3}$.

Foner. Sì. Lo stesso Hofstadter, che fu uno dei maggiori leader della cosiddetta "storiografia del consenso", negli anni '60 cominciò ad abbandonarla sotto l'impatto di ciò che stava accadendo nel mondo. Infatti si espresse in modo molto chiaro nel libro The Progressive Historians (Knopf, New York 1968) su cui lavorava quando ero un suo dottorando, affermando che il punto di vista della storiografia del consenso non era in grado di spiegare le cause della Guerra civile. Come si può pensare di sostenere che non ci sia alcuna divisione nella storia americana di fronte a 600.000 persone uccise in una guerra civile? Non è credibile! E poi emerse la questione razziale. Io stesso, negli anni ' 60 , non avrei mai pensato che sarebbe diventata un problema così importante. È ovvio che nella società americana stava emergendo una divisione profonda che veniva da lontano. L'ultimo libro di Hofstadter pubblicato dopo la sua morte, America at 1750 (Knopf, New Yorf 1971), fu uno studio pionieristico di storia sociale: trattava del conflitto sociale nell'America coloniale ed era assai diverso da ciò che aveva scritto prima. Hofstadter scrisse quel libro sotto l'impatto non solo degli eventi reali del suo tempo ma anche dei suoi dottorandi, molti dei quali esercitarono su di lui una notevole influenza. Fra questi c'erano Michael Wallace, Linda Kerber ${ }^{4}$ ed io. A proposito, non essendo veramente interessato all'insegnamento ma alla scrittura,

\footnotetext{
${ }^{3}$ Richard Hofstadter fu dal 1959 De Witt Clinton Professor di Storia americana alla Columbia University. Tra le sue opere: Social Darwinism in American Thought, 1860-1915 (University of Pennsylvania Press, Philadelphia 1944), The American Political Tradition and the Men Who Made It (Knopf, New York 1948), The Age of Reform: from Bryan to F.D.R. (Knopf, New York 1955), Anti-Intellectualism in American Life (Vintage Books, New York 1963).

${ }^{4}$ M. Wallace-E.G. Burnows, Gotham: A History of New York City to 1898, Oxford UP, New York 1999; L. Kerber, Women of the Republic: Intellect and Ideology in Revolutionary America, University of North Carolina Press, Chapel Hill 1980, Toward an Intellectual History of Women, University of North Carolina Press, 1997, No Constitutional Right to Be Ladies: Women and the Obligations of Citizenship, Hill an Wang, New York 1998.
} 
Hofstadter soleva dire: «Sono uno scrittore che insegna per guadagnarsi da vivere e che in realtà vorrebbe passare tutto il tempo a scrivere». Ma ciò che era straordinario nel suo modo di insegnare è che non cercava mai di imporre il suo punto di vista ai suoi studenti. Non esiste nessuna scuola di cui Hofstadter sia il capostipite, semplicemente ci sono i suoi seguaci, persone che studiarono con lui e ne furono influenzate.

Lorini. Quest'ultimo aspetto è vero anche per i tuoi ex studenti che hanno sviluppato progetti di ricerca diversi e non tutti hanno seguito i tuoi interessi. Tuttavia molti di loro portano avanti progetti diversi ma applicano un metodo chiaramente riconoscibile: è quello che stai dicendo?

Foner. È quello che cerco di fare. Come Hofstadter, non voglio imporre il mio punto di vista agli studenti. Ne ho avuti alcuni molto conservatori che attualmente insegnano. Quello che ho cercato di fare è di metterli in grado di esprimere al meglio le loro capacità di fare ricerca storica. Li criticavo soltanto quando non raggiungevano gli standard più elevati. E penso che questo valeva anche per Hofstadter: se guardate i suoi ex studenti, vedete che fanno ricerca nei campi più diversi e usano approcci diversi. Ho preso Hofstadter a mio modello per questo aspetto, ovviamente non per la sua mancanza d'interesse per l'insegnamento!

Frezza. Allora quali erano in quel periodo $i$ tuoi punti di riferimento, quando la vecchia cornice storiografica si era ormai rotta e l'idea di storia consensuale non reggeva più?

Foner. Dal punto di vista storico posso dire di aver avuto due punti di riferimento: uno era la mia famiglia perché sono cresciuto con un padre, Jack Foner, che faceva lo storico di professione, e uno zio, Philip, storico, e ho appreso dalle discussioni familiari un punto di vista diverso sulla storia rispetto a quello che si studiava a scuola. Appresi dell'esistenza di Frederick Douglass $^{5}$, di Du Bois 6 e di Tom Paine, conobbi molte cose sugli abolizionisti quando nessun insegnante parlava di queste figure e di questi movimenti. Nei nostri libri di testo degli anni '50 Douglass, che forse oggi è il personaggio più famoso nella storia americana, non era nemmeno menzionato. Io invece

\footnotetext{
${ }^{5}$ Del grande abolizionista nero F. Douglass (1818-1895) cfr.: L'indipendenza e la schiavitù (manifestolibri, Roma 1996), Memorie di uno schiavo fuggiasco (manifestolibri, Roma 2011), entrambi a cura di A. Portelli.

6 William Edward Burghardt Du Bois (1868-1963) fu uno dei più grandi intellettuali afroamericani, il primo a ricevere un Ph.D dalla Harvard University. Fu sociologo, storico, poeta, militante panafricanista e dei diritti civili. Tra le sue opere tradotte in italiano: Sulla linea del colore. Razza e democrazia negli Stati Uniti e nel mondo, a cura di S. Mezzadra, il Mulino, Bologna 2010, Negri per sempre. L'identità nera tra costruzione della sociologia e "linea del colore”, a cura di R. Rauty, Armando Editore, Roma 2008.
} 
ne sentivo parlare a casa. Non si trattava ovviamente di una lezione, apprendevo per osmosi. Non avevo pianificato di diventare uno storico poiché i miei interessi di allora riguardavano la scienza, la matematica, l'astronomia. Andai al college per studiare materie scientifiche ma poi mi accorsi di non essere bravo abbastanza in calcolo avanzato per poter andare avanti in quelle discipline...

Frezza. Che cosa pensavi quando a scuola gli insegnanti ti davano versioni completamente diverse della storia rispetto a quelle che sentivi a casa?

Foner. È difficile descrivere esattamente le mie sensazioni di allora perché da un lato ogni bambino pensa che la sua famiglia sia normale e anch'io pensavo che la mia lo fosse. Si parlava della situazione in Guatemala ${ }^{7} . .$. solo in seguito ho scoperto che allora non erano in molti a parlarne. Ma c'era anche dell'altro: crescevi con l'idea di appartenere a un mondo quasi segreto. Dopo tutto mio padre fu messo nelle "liste nere", il nostro telefono era controllato - probabilmente dal Fbi - e crescevo sapendo che c'erano certe cose di cui non si parlava al telefono o che non si potevano dire a scuola. In un certo senso si viveva una doppia vita, quello che Du Bois aveva chiamato «doppia coscienza» ${ }^{8}$. Ero un bambino assolutamente normale che giocava a baseball, usciva con gli amici, andava al cinema, ma che aveva anche un'altra vita. C'è un fatto che solitamente racconto: nel liceo l'insegnante di storia dette la sua versione della Ricostruzione come di un periodo veramente terribile e io la contestai dicendo: «Non sono d'accordo». Allora lei disse: «Bene, se non ti piace ciò che ho detto, domani sarai tu a fare una lezione sulla Ricostruzione». Con l'aiuto di mio padre preparai questa lezione basata su Black Reconstruction di Du Bois9: mi presentai in classe discutendo su come la lotta per la democrazia non fosse semplicemente quella contro la corruzione e il malgoverno e alla fine della lezione l'insegnante disse: «Bene, facciamo votare tutta la classe sulle due lezioni, su chi di noi due abbia ragione». Ovviamente tutti gli studenti, con la sola eccezione di Neal, il mio migliore amico, votarono a favore dell'insegnante. Così vivevo in un modo diverso. Probabilmente decisi allora che avrei in seguito provato che la mia insegnante aveva torto. Così, trent'anni dopo, scrissi un libro di 800 pagine sulla Ricostruzione ${ }^{10}$ per dare la prova alla signora Berriman che io avevo ragione e lei sbagliava.

\footnotetext{
7 Il riferimento è alla giunta militare andata al potere nel 1954 in Guatemala grazie alla Cia.

${ }^{8}$ Il riferimento è al concetto di double consciousness che Du Bois elabora nel suo capolavoro The Souls of Black Folks (Bantam Classic, New York 1903, trad. it. Le anime del popolo nero, Le Lettere, Firenze 2007).

9 W.E.B. Du Bois, Black Reconstruction in America 1860-1880 (1935) Free Press, New York 1999.

10 E. Foner, Reconstruction: America's Unfinished Revolution, 1863-1877, Harper and Row, New York 1988. Il libro ha ricevuto il Bancroft Prize, il Parkman Prize, il Los Angeles Time Book Award.
} 
Sapete che Long Beach, il luogo dove sono cresciuto, era abbastanza liberale in piena guerra fredda, era una cittadina che votava democratico come gran parte dei suburbs. C'era qualche insegnante con una vaga visione progressista delle cose. Fu solo quando andai alla Columbia University e cambiai il percorso di studi facendo diventare la storia la mia materia principale, che incontrai James Shenton ${ }^{11}$, che non ha scritto molto ma era veramente un grande insegnante. Lui stesso era un ribelle. Siamo negli anni '60, siamo ancora nella guerra fredda, e Shenton insegnava Du Bois, John Brown, esponeva un punto di vista diverso della storia americana, parlava di razza e di schiavitù. Nei suoi corsi si sentivano molte cose di cui negli altri non si parlava. I suoi corsi mi influenzarono molto, ma quando scrissi la tesi di dottorato lo storico che mi influenzò più di ogni altro fu Eugene Genovese ${ }^{12}$. Mi è difficile ammetterlo perché a un certo punto penso che sia andato fuori binario, ma il suo Political Economy of Slavery (1965) fu un testo fondamentale che lessi agli inizi dei miei studi di dottorato alla Columbia. Fu un libro che in un certo senso mandò in frantumi la storiografia precedente; introdusse realmente il marxismo nel modo americano di pensare la storia, dopo che era stato completamente eliminato, ma lo fece in un modo complesso. Non era il vecchio marxismo professato dal Partito comunista di mio zio Phil Foner e di altri che fecero una quantità enorme di ricerca ma la rinchiusero in uno schema estremamente ristretto e dottrinario. Genovese ci offriva un marxismo assai più aperto e critico: penso che fosse assai più valido, facendo conoscere quel versante dell'ideologia che era stato eliminato e che io studiai per la tesi di dottorato che poi divenne il mio primo libro ${ }^{13}$.

Nel momento in cui l'ideologia del consenso si stava sgretolando nella società, scontrandosi con la storia, ecco che arrivò Genovese e l'inizio della cosiddetta "storia della nuova sinistra". Il suo libro ci insegnò un nuovo passato, uscì insieme ai saggi di Jesse Lemisch History from the Bottom Up (University of South Carolina Press, Columbia 1966). Ero stato in Inghilterra a studiare dal 1963 al 1965 e lì incontrai le opere di Hobsbawm e di Thompson. Sono davvero molto contento che come studente alla Columbia University avessi ricevuto

${ }^{11}$ James Shenton era molto popolare tra gli studenti della Columbia dove iniziò a insegnare nel 1951. Ha ricevuto numerosi premi prestigiosi per le sue qualità di docente. Come "intellettuale pubblico" negli anni ' 60 Shenton fece un corso di 76 ore per la televisione pubblica intitolato The Rise of the American Nation. Tra le sue pubblicazioni: History of the United State to 1865 (Doubleday, New York 1963), History of the United States from 1865 to the Present (Doubleday, New York 1964).

12 Tra le opere di E. Genovese: The Political Economy of Slavery: Studies in the Economy and the Society of the Slave South (Pantheon Books, New York 1965), The World the Slaveholders Made: Two Essays in Interpretation (Pantheon Books, New York 1969), Roll, Jordan, Roll: The World the Slaves Made (Pantheon Books, New York 1974). Negli anni '90 Genovese abbandonò la prospettiva marxista e anche liberale di sinistra, diventando un conservatore tradizionalista.

${ }^{13}$ Si tratta di Free Soil, Free Labor, Free Men: The Ideology of the Republican Party Before the Civil War, Oxford UP, New York 1970. 
una formazione storica assolutamente tradizionale, per niente eterodossa. Presidenti, politica, leader, ma assolutamente fantastica! Credo che tutti debbano avere quel tipo di formazione di base, non credo che si possa studiare solo storia sociale poiché è necessario conoscere il retroterra politico. Poi, da quella base, si possono ampliare gli orizzonti. Allora vi erano molti limiti, la storia della gente comune non faceva parte della narrativa storica. Ma avendo una solida base storica tradizionale si poteva ampliarla ed è ciò che feci. La mia tesi di dottorato era in realtà un tipo di lavoro piuttosto convenzionale, alla Hofstadter. Il mio libro successivo, Tom Paine and Revolutionary America ${ }^{14}$, fu del tutto differente: trattava di movimenti sociali e dell'ideologia politica radicata in questi movimenti. Potrebbe suonare un'affermazione di uno pieno di sé e non voglio essere frainteso, ma pensate a Picasso: la gente è scioccata quando guarda le opere di Picasso e vede che le prime erano molto tradizionali.

Frezza. Riguardo al marxismo, che cosa pensi di aver preso dall'ideologia marxista?

Foner. La gente mi chiede: «Sei marxista?» e io rispondo: «Bene, che cos'è un marxista? Ditemi che cos'è e vi dirò se lo sono anch'io!».

Lorini. Lo stesso problema di definizione può emergere rispetto al socialismo...

Foner. Esattamente. Nel discorso politico americano il termine «marxista» è così carico di significato e di pregiudizi che se uno si dichiara marxista scatena un dibattito gigantesco. Quello che voglio dire è questo: non sono marxista, sono marxisant. Sono nell'orbita del marxismo ma non un marxista tout court; in altre parole, sono influenzato dal marxismo. In realtà Marx direbbe che io non sono marxista perché non gli piaceva mai ciò che si poteva fare con la sua ideologia. Allora, che cos'è il marxismo? È una modalità di pensiero, è un'analisi critica...

Lorini. Riguarda la categoria di conflitto...

Foner. Esattamente. Quando John Swinton ${ }^{15}$, il giornalista del movimento operaio americano - che aveva un giornale negli anni ' 80 dell' 800 - si recò

${ }^{14}$ E. Foner, Tom Paine and Revolutionary America (1976), Oxford UP, New York 2005, è uno studio esemplare dell'intreccio tra storia politica, intellettuale e sociale nella lotta per l'indipendenza americana. È centrato sulla figura di Paine, il suo impatto sul movimento rivoluzionario, la sua capacità di usare un linguaggio semplice ed esortativo per delineare un'idea radicale di repubblica nel celebre opuscolo Common Sense (1776).

15 John Swinton (1829-1901), di origine scozzese, negli anni '60 dell'800 scrisse editoriali per il «New York Times» e il «New York Sun». Il suo «John Swinton's Paper» fu uno dei più importanti giornali del movimento operaio negli anni '80. 
in Inghilterra e intervistò Marx, gli chiese: «Che cosa prevede per il futuro?», Marx non disse «prevedo l'avvento del socialismo e del comunismo», ma disse: «Lotta: è questo che vedo nel futuro». Quindi il marxismo è una teoria del conflitto, una teoria delle classi come chiave di lettura e cornice dell'analisi storica, una teoria di economia politica, vale a dire dell'interconnessione delle forze politiche ed economiche. Tutte queste sono delle intuizioni di grande valore che penso siano essenziali per la comprensione della società e della storia. Però la storia non si muove in una direzione predeterminata. Io cerco di farmi influenzare da cose di vario genere. Per esempio, penso che l'introduzione del femminismo nell'analisi storica sia qualcosa di profondamente diverso su cui Marx non avrebbe molto da dire. Ma io credo che il femminismo sia stato cruciale, che abbia cambiato in molti aspetti il modo in cui pensiamo la storia. Così cerco di essere aperto a molte cose, ma non riesco a capire come si possa essere storici e non conoscere il marxismo. Poi si può fare del marxismo ciò che si vuole e questo è un problema in America dove, a causa della guerra fredda, il marxismo come impresa intellettuale è caduto in disgrazia, oppure è stato associato al comunismo sovietico e quindi messo totalmente in disparte. Chiunque abbia interesse alla vita americana di oggi, specialmente negli ultimi 10/15 anni, con la crisi economica e la globalizzazione, deve per forza rileggere i libri di Marx.

Lorini. Sì, certo. C'è una tendenza nelle università europee a dirigere la ricerca degli studenti su temi minori, invece di guidarli verso temi più importanti come hanno fatto $i$ tuoi ex studenti, a cercare una nuova sintesi...

Foner. Io cerco di spingere i miei studenti ad impegnarsi nelle ricerche di cui li credo capaci. Ho avuto studenti che ho cercato di indirizzare verso obiettivi più limitati, in rapporto alle loro capacità. Tu, Sandra, hai avuto la fortuna di appartenere a un gruppo di studenti molto dotati che lavoravano alla Columbia alla fine degli anni '80 e agli inizi degli anni '90. Si trattava di un gruppo davvero rimarchevole, in parte grazie alle loro proprie capacità in parte grazie a quel particolare momento storico; per questo, grazie anche a quel particolare momento storico, l'ho indirizzato verso obiettivi importanti ${ }^{16}$. D'altra parte una tesi di dottorato deve essere un progetto fattibile, non può essere ovviamente un libro che sintetizzi nuove prospettive storiche, ma deve collocarsi in un quadro storiografico significativo.

Frezza. Potresti farci qualche esempio relativo all'influenza che la storiografia femminista ha avuto sul tuo lavoro?

\footnotetext{
16 Alcuni contributi di questo primo gruppo di studenti sono raccolti in Contested Democracy: Fredom, Race, and Power in American History, edited by M. Sinha and P. von Eschen, Columbia UP, New York 2007.
} 
Foner. La nozione di femminismo non esisteva nemmeno quando ero ragazzo, ma i vecchi comunisti, nel loro modo incerto e in gran parte sbagliato erano in un certo senso "femministi", vale a dire credevano nelle donne. Io sono cresciuto con la consapevolezza che mia madre aveva un lavoro, una carriera, una professione: era un'artista. Noi non vivevamo in una casa con una forte connotazione di "genere", a parte il fatto che mia madre si occupava quasi di tutte le faccende domestiche. Si dava per scontato che quello che mia madre faceva era altrettanto importante di quello che faceva mio padre. Non vi era l'idea dell'uomo capofamiglia e della donna subordinata. Sono cresciuto in questo modo, nessuno mi doveva spiegare queste cose, semplicemente era così, questo era il modo secondo il quale pensavo si dovesse vivere. A quell'epoca, negli anni '50, l'ideologia corrente era sopratutto basata sul fatto che le donne dovessero occuparsi della casa... A proposito: mia madre era una cuoca tremenda! Credo che fosse una forma di ribellione. Non mi resi conto che mangiare fosse qualcosa di piacevole fino a quando non andai alla Columbia. Il professor Shenton era solito portare fuori a cena gli studenti dei suoi seminari. Perciò è stato per via di Shenton che ho imparato che a New York c'erano ristoranti magnifici, francesi, italiani, spagnoli, cinesi e che il cibo può essere un godimento. Non si doveva mangiare soltanto per sopravvivere...

Ma torniamo all'impatto del femminismo sul mio lavoro. Nel mio libro sulla Storia della libertà americana ${ }^{17}$ il femminismo ha avuto un ruolo cruciale nel delineare il dibattito che si venne articolando sul tema della libertà. Ha suscitato molta sorpresa il fatto che nel libro ci sia molto su questo tema. Ritengo che la lotta delle donne per l'eguaglianza in forme diverse e in periodi differenti sia stata assolutamente centrale per lo sviluppo della nozione di libertà, sia che si trattasse delle sorelle Grimkè 18 negli anni ' 30 dell' 800 o di Victoria Woodhull19 negli anni '70 o di persone come Emma Goldman ${ }^{20}$ e Margaret Sanger ${ }^{21}$ negli anni '10 del '900, fino ad arrivare agli anni '60. Ri-

17 E. Foner, Storia della libertà americana, Donzelli, Roma 2000. Per una discussione sul volume cfr. «Passato e presente», 19 (2001), n. 53, pp. 15-40.

${ }^{18}$ Sarah e Angelina Grimké, provenienti da una famiglia del Sud proprietaria di schiavi, furono leader del movimento femminista e abolizionista che nel 1848 portò alla stesura della $\mathrm{Di}$ chiarazione dei sentimenti di Seneca Falls, cfr. R. Baritono (a cura di), Il sentimento della libertà. La Dichiarazione di Seneca Falls e il dibattito sui diritti delle donne negli Stati Uniti di metà Ottocento, La Rosa editrice, Torino 2001.

19 Victoria Woodhull, attivista radicale dei diritti delle donne, si occupò di uguaglianza sessuale, di amore libero, occupazione femminile e diritto di voto.

${ }^{20}$ Emma Goldman, emigrata negli Stati Uniti a soli 15 anni da San Pietroburgo, è una figura di primo piano nella storia del movimento anarchico internazionale e dell'emancipazione femminile. Tra i suoi scritti: Anarchia, femminismo e altri saggi (La Salamandra, Milano 1976), La sconfitta della Rivoluzione russa e le sue cause (La Salamandra, Milano 1977), Autobiografia. Vivendo la mia vita (4 volumi usciti tra il 1980 e il 1993 per La Salamandra e Zero in Condotta).

${ }^{21}$ L'infermiera Margaret Sanger, fondatrice dell'associazione Planned Parenthood, divenne la principale attivista del movimento per il controllo delle nascite negli Stati Uniti agli inizi del ' 900. 
tengo che il movimento femminista abbia contribuito più di ogni altro movimento sociale a porre la nozione di libertà personale come tema cruciale rispetto a come noi la prospettiamo oggi. Washington e Jefferson parlavano del controllo della persona stessa come di un elemento fondamentale nel rendere la nozione di libertà un obiettivo politico, un tema di interesse pubblico; ma le femministe hanno introdotto la nozione di libertà nelle parti più intime della vita privata e hanno argomentato come vi siano relazioni di potere e di disuguaglianza persino nella camera da letto, nei diversi aspetti della vita quotidiana. Personalmente ritengo che questo sia un elemento di fondamentale importanza analitica.

Io non mi occupavo di questi aspetti nei miei primi lavori, ma le opere di storiche femministe come Joan Scott ${ }^{22}$ o Carol Gilligan ${ }^{23}$ o molte altre come Ellen Du Bois ${ }^{24}$, hanno profondamente influenzato il mio lavoro oltre ad aver contribuito in modo rilevante alla comprensione della storia americana. Infine penso che si debba mantenere una mentalità aperta, che si debba sempre imparare quando si studia la storia. Non si può restare all'interno della stessa cornice interpretativa dal momento che lo studio della storia è sempre in cambiamento. È necessario avere una mentalità aperta così da accogliere aspetti interessanti e utili perfino nel caso di mode che magari si rivelano senza sbocchi. Penso ad esempio allo studio della whiteness ${ }^{25}$ che ad un certo punto era diventato un tema di grande interesse e poi è crollato per la propria inconsistenza. Tuttavia ha fornito elementi utili all'analisi storica. In conclusione, ritengo che si debba fare attenzione a idee nuove che abbiano una rilevanza nello studio della storia senza però lasciarsi condizionare dalle ultime mode. A volte queste iniziano in modo brillante ma poi si bloccano in una forma di ideologia che non fa che ripetersi all'infinito.

Lorini. Vi è una domanda che voglio porti e che può essere interessante per un pubblico europeo. Hai detto che la storia viene riscritta generazione

22 Tra le sue opere: Gender and the Politics of History (Columbia UP, New York 1988), Feminism and History (Oxford UP, New York 1996); Donne, lavoro e famiglia nell'evoluzione della società capitalistica (coautrice L.A. Tilly, De Donato, Bari 1981); Il "genere": un'utile categoria di analisi storica, «Rivista di storia contemporanea», 2 (1987), n. 4.

${ }^{23}$ Si ricordano In a Different Voice (Harvard UP, Cambridge 1982), Between Voice and Silence: Women and Girls, Race and Relationships (Harvard UP, Cambridge 1997), The Deepening of Darkness: Patriarchy, Resistance, and Democracy's Future (coautore D.A.J. Richards, Cambridge UP, Cambridge 2009).

24 Tra cui segnaliamo Through Women's Eyes: An American History with Documents (coautrice L. Dumenil, St. Martin's Publishers, Bedford 2005), Woman Suffrage and Women's Rights: Essays (New York UP, New York 1997), Feminism and Suffrage: The Emergence of an Independent Women's Movement in America 1848-1869 (Cornell UP, Ithaca-New York 1978).

${ }_{25}$ Per un esame critico degli studi sulla whiteness cfr. P. Kolchin, Whiteness Studies: The New History of Race in America, «The Journal of American History», 89 (2002), n. 1, pp. $154-73$. 
dopo generazione alla luce delle nuove ricerche storiche e archivistiche. In Italia dobbiamo osservare come il termine «revisionismo» abbia una connotazione negativa...

Foner. Succede anche qui. Questo problema rappresenta la differenza maggiore che esiste tra il nostro lavoro di studiosi e quello che il pubblico in generale definisce come storia. Il termine «revisionismo storico» implica l'idea di un abuso di un'analisi critica; è come se qualcuno facesse a pezzi verità consolidate, confondendo le idee a tutti. Il revisionismo storico non è altro che il lavoro che noi svolgiamo, quello di scrivere la storia utilizzando idee nuove, nuovi punti di vista, nuove metodologie, nuove domande. Se non si facesse questo che interesse ci sarebbe nello studio della storia? Che senso avrebbe scrivere nuovi libri? Il problema è che il pubblico in generale non capisce questo e quindi è sempre stato un tema oggetto di discussione. Andate a leggere il libro di Carl Becker del 193526. Non c'è nulla di nuovo in questo dibattito. Nel suo Everyman His Own Historian il tema centrale riguarda proprio il fatto che la storia non è soltanto una raccolta di fatti e verità. La stessa selezione dei fatti è basata sull'interpretazione dello storico. Che cos'è un fatto? Questo è il motivo per cui ci sono affinità tra lo scrivere un libro di storia e una fiction. Sono ambedue frutto di un atto di immaginazione personale ma lo storico impone un ordine negli accadimenti storici.

Lorini. Dunque la storia non fa parte delle scienze sociali...

Foner. Non è una scienza sociale. Il termine «scienza» non dovrebbe mai essere applicato alla storia. Si tratta di una creazione umana. Edward H. Carr nel suo libro Sei lezioni sulla storia lo chiarisce molto bene.

Frezza. D'altra parte lo storico impone la propria interpretazione dei fatti ma deve cercarne le prove nei documenti, negli archivi.

Foner. Certamente. Non si tratta di fiction perché si devono giustificare le proprie interpretazioni e vi sono standard professionali ai quali è necessario attenersi. Abbiamo l'apparato delle note. Il pubblico può dire: «Non credo a questo». Bene, può andare a verificare. Molti lavori storici non sono convincenti e per questo motivo sono simili a un romanzo. Da questo punto di vista la narrazione storica può considerarsi affine a un lavoro scientifico, perché deve poter essere verificata, deve essere riproducibile. Non credete che Jefferson abbia detto questo? Andate a guardare nelle sue opere dalle quali ho tratto questo discorso.

\footnotetext{
26 Tra le opere di Carl Becker: The Beginnings of the American People (Houghton Mifflin Company, New York 1915), The Eve of the Revolution (Yale UP, New Haven 1918), The Declaration of Independence (Harcourt, Brace and Co, New York 1922), The Heavenly City of the Eighteenth Century Philosophers (Yale UP, New Haven 1932), Every Man His Own Historian (F.S. Crofts and Co, New York 1935).
} 
Frezza. Certo, ma qualcuno potrebbe dire: «D'accordo ma in questo altro punto Jefferson ha detto cose molto differenti»...

Foner. Non c'è dubbio, la cosa più semplice sarebbe scrivere una storia come vorresti che fosse. Potrei scrivere facilmente un libro con un apparato critico basato su documenti reali per dimostrare come i proprietari di schiavi li trattavano bene. Si potrebbe trovare un riscontro obiettivo su questo. Ma qualcuno potrebbe osservare: «Un momento, sta ignorando il $90 \%$ di quanto risulta dalla ricerca storica!». Altri lo scopriranno e lo segnaleranno. Pertanto non si tratta solo del problema di utilizzare i fatti, si tratta anche di quelli che non si prendono in considerazione, della cornice nei quali si inseriscono. Non si tratta soltanto di documenti nuovi. I documenti sono già noti. Si tratta di nuove domande, di nuovi approcci di fronte ai documenti. Quando ho scritto il libro su Lincoln ogni singolo aspetto della sua figura era già noto. Ho proposto un'interpretazione nuova, un nuovo contesto, una maniera nuova di riflettere rispetto ad alcuni aspetti della sua carriera e il pubblico è in grado di giudicare facendo un raffronto con i documenti. Perciò sono gli interrogativi, è l'intero approccio che è nuovo. Per questo motivo negli anni '50 non c'era nessuno che scriveva sulla storia delle donne. Semplicemente non era presente nell'insegnamento universitario, non vi erano corsi o tesi magistrali o di dottorato. Prima degli anni ' 70 gli storici si interessavano alle figure pubbliche dei leader nel settore economico, politico, nelle campagne elettorali, e i loro studi non includevano le donne come soggetti. Quando gli interessi degli storici si sono modificati, le donne sono entrate nell'arena pubblica.

Frezza. Nel discorso di chiusura del tuo mandato di presidente dell'American Historical Association parlasti molto del tema della globalizzazione rispetto alla storia degli Stati Uniti d'America ${ }^{27}$. Si trattava di una prospettiva nuova, di un modo nuovo di pensare alla storia che non era così presente vent'anni fa. Puoi farci qualche esempio su questo tema?

Foner. L'analisi della storia americana in un contesto internazionale non è cosa del tutto nuova, ma è oggi molto più presente. Thomas Bender ${ }^{28}$ si è particolarmente impegnato su questo versante.

Lorini. Nel libro A Nation among Nations?

Foner. Sì e nelle molte occasioni di discussione pubblica su questo tema. Ritengo che non sia stato un libro di successo - e Bender ne è consapevole -

27 E. Foner, American Freedom in a Global Age. Presidential Address, «The American Historical Review», 106 (2001), n. 1, pp. 1-16.

${ }_{28}$ Di T. Bender cfr. la cura del volume Rethinking American History in a Global Age, University of California Press, Berkeley 2002. Sull'internazionalizzazione della storia americana: A Nation Among Nations: America's Place in World History, Hill and Wang, New York 2006. 
anche se è fortemente innovativo. Non vi è ancora una storiografia consistente che permetta di scrivere un libro di questo tipo in modo del tutto soddisfacente. Ma è meglio fare uno sforzo in questa direzione piuttosto che scrivere un saggio dicendo: "Questo è ciò che gli storici dovrebbero fare»; è molto più difficile dire: «Ecco, sto lavorando in questa direzione». Credo che il grande merito di Bender consista nel togliere di mezzo tutto quel vasto settore di tanta storia americana, dalle fondamenta incerte, che va sotto il nome di «eccezionalismo americano» e che fa parte integrante della nostra cultura e della nostra politica. Tutti vi fanno riferimento, compresi Obama e Romney: si deve parlare di eccezionalismo americano se si partecipa alla vita pubblica in America. Per gli storici significa che non è necessario conoscere niente degli altri paesi dal momento che noi siamo così diversi. Il resto del mondo è per noi irrilevante; naturalmente abbiamo rapporti con gli altri paesi ma non è necessario conoscere la loro storia in quanto è così diversa dalla nostra che non ha alcun peso. Tutto questo genera populismo, produce ignoranza e soprattutto affermazioni che non hanno nessun fondamento nei fatti reali: per esempio, si dice che gli Stati Uniti sono conservatori mentre l'Europa come insieme è radicale e rivoluzionaria e la classe operaia in Europa si batte sempre contro lo stato mentre qui non succede. Tutto questo è ridicolo ma lo si trova in molta letteratura passata. Naturalmente ogni paese ha la sua storia particolare, la storia della Francia non è la stessa cosa della storia della Cina che a sua volta non è simile a quella dell'Argentina e degli Stati Uniti.

Quando parliamo dell'eccezionalismo americano non stiamo solo affermando «L'America è diversa dagli altri paesi», ma stiamo dicendo: «Vi è un'altra storia altrove e poi vi è la storia americana. Noi americani siamo diversi da tutto il resto del mondo e questo significa che vi è un percorso storico al quale noi non partecipiamo». Detto questo è necessario però osservare che molti o forse la maggior parte dei processi storici che hanno segnato la storia americana sono processi globali, non importa se si tratti delle esplorazioni, della schiavitù, di flussi internazionali di capitale o processi di migrazione e di urbanizzazione. Tutto questo accade dovunque. Io non incoraggio i miei studenti a preparare una tesi di dottorato su questi temi perché richiede una familiarità con tanti tipi diversi di storie e di storiografie. Per esempio mi piacerebbe comparare la schiavitù americana con quella brasiliana. Ma anche dopo aver studiato la letteratura sulla schiavitù brasiliana è davvero difficile trovare un punto di vista tale da comprendere i loro dibattiti, quelli che devono essere inquadrati nel contesto brasiliano, che è condizionato dalla loro politica, dalla storia del loro paese con la quale non abbiamo molta dimestichezza. Perciò non è solo questione di scegliere un paio di libri: come possiamo orientarci su ciò che è storicamente valido o meno? A parte il fatto che in questo paese non abbiamo dimestichezza con le lingue straniere, come si può affrontare una storia globale o una storia globale americana quando tutto questo riguarda solo l'area anglofona? Il nostro sistema educativo è orientato in senso contrario rispetto a un progetto intellettuale rivolto ad acquisire una visione globale. Perciò non è così semplice affrontare una storia globale, ma 
come nozione di base essa ha sicuramente un effetto salutare rispetto al modo con il quale pensiamo alla storia americana.

Lorini. Negli studi internazionali di storia americana le cose stanno cambiando, ad esempio ponendo in discussione la definizione stessa di storia americana che viene ora chiamata Storia degli Stati Uniti: si tratta del tentativo di dare spazio all'idea che vi sono molte Americhe.

Foner. Giusto. Ci sono molte Americhe. Tuttavia è vero che nella lingua anglo-americana America significa gli Stati Uniti. Si tratta di una nozione imperiale.

\section{Lorini. Questo ci riporta all'eccezionalismo americano...}

Foner. Esattamente. È una nozione imperiale. Quando mi occupo di storia americana tutti sanno cosa voglio dire. Non sto trattando del Messico o di Cuba. Naturalmente questo implica una considerevole dose di ignoranza anche riguardo ai paesi che sono ai nostri confini. Forse qualcuno sa qualcosa del Canada? Il Canada è una presenza importante nella nostra geografia ma nessuno ne sa nulla, anche se la lingua è prevalentemente la stessa. I confini geografici degli Stati Uniti riducono anche la nostra prospettiva storica.

Frezza. Forse una delle cause di questo è dovuta al fatto che trattandosi di un grande paese, quasi un continente, vi è una tendenza a mantenere lo sguardo al proprio interno.

Foner. Ma dovrebbe esserci una prospettiva rivolta verso l'esterno! La storia dell'impero sta sviluppandosi secondo la moda post-coloniale. Credo che tutte queste tendenze arricchiscano la storia americana. Nonostante tutto oggi la storia americana ha raggiunto un livello di maggiore sofisticazione, di maggiore complessità. È quasi impossibile stare al passo con la proliferazione di libri, articoli, saggi online. La letteratura sulla storia americana continua a crescere. In un certo senso, paradossalmente, perché da un lato vi è una proliferazione di lavori ma questo incoraggia una maggiore specializzazione. Per esempio, non riesco a tenere il passo con tutto quello che esce sulla storia coloniale, perciò mi sono concentrato su quanto esce a proposito della Guerra civile e della schiavitù.

Lorini. Un altro tema che credo sia importante e che viene fuori spesso nel tuo lavoro è il rapporto fra storia e memoria. La storia riguarda la riflessione critica su storiografie contrapposte mentre la memoria in un certo senso è una lotta per il possesso del passato. Tu hai organizzato molti eventi pubblici nei quali hai assunto posizioni critiche e il pubblico reagiva dicendo: «Non puoi sapere, questa è la nostra memoria». Penso ai temi che riguardano il Sud, la bandiera della Confederazione e cose simili. Ritieni an- 
cora che si tratti di un rapporto importante da analizzare visto che gli storici si occupano anche di storia della memoria?

Foner. Certamente. Vi sono due aspetti molto diversi che vengono messi insieme sotto la categoria della memoria. Forse è una buona idea tentare di separarli. Un aspetto è infatti la memoria: persone che hanno sperimentato eventi e li ricordano, memorie ad esempio dei veterani della Seconda guerra mondiale, che ne discutono. Quelle persone hanno vissuto quegli eventi e questa è la memoria come io la intendo: «Mi ricordo di quegli eventi». Questa ha un impatto sulla percezione pubblica della storia. Negli anni ' 90 vi è stata una controversia su una mostra che doveva essere allestita dallo Smithsionian Institute di Washington (DC), sullo sganciamento della prima bomba atomica su Hiroshima, e alcuni gruppi o associazioni protestarono perché ritenevano che la mostra avesse un carattere troppo revisionista. L'esposizione offriva argomentazioni pro e contro l'uso della bomba atomica e intendeva evidenziare alcune delle conseguenze devastanti dell'evento. Gruppi di veterani lamentavano che non fosse abbastanza celebrativa e infine non si fece. In sostanza lo Smithsonian Institute non voleva essere trascinato in quella polemica. Le persone che hanno vissuto quegli eventi ne reclamano simbolicamente il possesso e questo diventa un problema per la storia americana più recente. Una cosa simile è avvenuta per il sito del World Trade Center a New York, non tanto sulla memoria ma sull'esperienza condivisa. Vi era il progetto di costruire un piccolo museo sulla storia della libertà negli Stati Uniti ma i parenti delle vittime dell'11 settembre ne volevano il controllo totale e non volevano che emergesse alcuna forma di critica verso gli Stati Uniti. Doveva essere un museo sulla libertà ma a loro avviso «la libertà è perfetta in questo paese e lo è sempre stata». Alla fine il progetto venne cancellato perché gli storici obiettarono che questa posizione era assurda.

Questo è un tipo di memoria ma ciò di cui Sandra parla non è la memoria, è la coscienza pubblica relativa alla storia. Non mi piace il termine memoria perché suggerisce ciò che si è effettivamente sperimentato. Nessuno che sia in vita oggi ricorda la Guerra civile. Può averne delle idee, delle immagini, ma quelle idee non derivano dalla memoria personale bensì dalla cultura, dai film, dai libri di storia, da qualsiasi altra fonte. Questo è il rapporto fra la storia come disciplina accademica e la coscienza pubblica della storia. Questo è un dato molto importante e il pubblico non dovrebbe avere un interesse di parte rispetto alla memoria di alcuni eventi storici. Sono stato coinvolto nella ristrutturazione del centro visitatori di Gettysburg, il campo di battaglia della Guerra civile dove passano milioni di visitatori. Il pubblico vuole vedere ciò che gli è familiare. Se vanno a Gettysburg, per esempio, e vedono che la schiavitù è messa in evidenza, come adesso facciamo, come la causa principale della Guerra civile, alcuni dicono «bene!» altri invece: «no, stai insultando i miei antenati, la mia memoria! Non vogliamo sapere della schiavitù, raccontaci solo della battaglia, i movimenti delle truppe e chi li comandava». Questi gruppi di persone hanno interessi di parte. 
Facciamo un altro esempio completamente differente, la battaglia di Little Big Horn e il generale Custer. In quel luogo vi è un parco nazionale; fino a poco tempo fa la scena ruotava tutta intorno a Custer che fu ucciso e ai suoi uomini che vennero sconfitti. Cosa ne era degli altri protagonisti di quell'evento? Si presume che si debbano introdurre gli indiani, per esempio il capo dei Sioux Sitting Bull, Crazy Horse e, ovviamente, dobbiamo raccontare la loro storia. Ma come la si racconta? Vi sono gli storici ma vi sono anche le tribù indiane e molte sono ancora presenti e vive. Hanno la loro tradizione orale che emerge e non si tratta della storia come la intendiamo noi: si tratta di memoria e tradizione sociale. Gli storici dicono: «Attenti, anche questo è ciò che avvenne allora». Ma le tribù indiane hanno una loro concezione rispetto a quei fatti. Come si può presentare al pubblico tutto questo? Evidentemente qui ci sono delle storie diverse. Vi è quella di Custer, quella dei Sioux, quella degli storici? Tutto questo genera confusione e i visitatori lo rifiutano e chiedono invece: «Ditemi soltanto quello che è successo a Custer nella sua ultima impresa. Non voglio ascoltare tutto questo impasto fuorviante e dicerie moderne». Diventa molto complicato perché le persone hanno differenti punti di vista. Io ritengo che quando le mostre vengono allestite in questi luoghi si tratta come di scrivere un libro, soltanto che viene fatto con altri strumenti.

\section{Lorini. Per un pubblico più vasto?}

Frezza. Sì, ma è l'interpretazione degli storici: «Mi hanno impiegato per fare questo e io vi propongo la mia interpretazione della storia. Se chiamavano qualcun altro egli avrebbe proposto una mostra differente». Diciamo all'inizio delle nostre mostre ${ }^{29}$ : «E scritto sul muro: questa mostra è un'interpretazione del periodo della Guerra civile, della Ricostruzione; è fondata sulla storiografia più recente, sulla ricerca dei curatori della mostra ma è una spiegazione e ve ne sono molte altre disponibili». A un certo punto, quando stavamo preparando la mostra sulla Ricostruzione abbiamo avuto l'idea di proporre le diverse interpretazioni. Ve ne era una vecchia secondo la quale la Ricostruzione era stata terribile, e conteneva un punto di vista razzista. Decidemmo alla fine di non farlo perché alcuni avrebbero potuto pensare che quella era la storia di oggi. Così abbiamo deciso di prenderci la responsabilità di affermare ciò che riteniamo sia la storia, fondata sulla nostra ricerca e sul nostro sapere, sulla nostra competenza. Per questo il pubblico viene, per l'expertise. Alcune persone hanno detto: «Non mi piace tutto questo, questo non è quello che ho imparato a scuola». Altri hanno detto: «Bello, ho imparato qualcosa di nuovo. Questa è vera formazione culturale, per questo sono

${ }^{29}$ Foner ha curato con Olivia Mahoney due esposizioni di storia americana che hanno ricevuto molti premi: "A House Divided: America in the Age of Lincoln" (Chicago Historic Society, 1990) e "America's Reconstruction: People and Politics after the Civil War" (Virginia Historical Sociey, 1992 e poi in molte località statunitensi). 
qui, per imparare qualcosa di nuovo». Si può essere coinvolti in controversie in maniera crescente perché gruppi di potere come i veterani vanno al Congresso: i rappresentanti sono in buona misura ignoranti e i veterani si fanno sentire e a quel punto parte anche la ricerca di fondi. Il potere unito al denaro rappresenta qualcosa di diverso dalla conoscenza storica.

Lorini. Ti sei anche adoperato per fare cambiamenti importanti a Disneyland, dove ora si parla di schiavitù grazie al tuo lavoro. Ricordo che ti capitò di andare a visitare Disneyland quando tua figlia Daria era piccola e ti rendesti conto che ciò che milioni di visitatori imparavano sulla storia americana era alquanto improprio. Sarebbe interessante approfondire questo aspetto.

Foner. Quando mia figlia aveva 4 anni la portai a Disneyland, in California, dove tra le altre cose ci fermammo a vedere la mostra "Incontra il Sig. Lincoln" che consisteva in una proiezione di diapositive sulla Guerra civile e in un robot raffigurante Lincoln che presentava alcuni estratti dai suoi famosi discorsi. Nella proiezione di diapositive non c'era nessuna immagine che raffigurasse una persona di colore e nei discorsi la schiavitù non era nemmeno menzionata. Mandai una lettera a qualcuno di mia conoscenza della direzione del parco disneyano dicendo: «Non mi aspetto che il castello della Bella Addormentata sia una ricostruzione storica "accurata", ma se pretendete di rappresentare la storia della Guerra civile dovete farlo in un modo che sia meno datato». Mi fu subito chiesto di revisionare la presentazione, ma di dare prima un'occhiata alla Sala dei Presidenti a Disneyworld in Florida. Là avevano i robot di tutti i presidenti americani e proiettavano un filmato di 5 minuti sulla storia americana. Anche questo era completamente datato, come se tutti i problemi fossero stati già risolti al momento della nascita della Costituzione. Così riscrissi il testo enfatizzando la lotta costante per realizzare le idee della Rivoluzione, idee che non erano ancora completamente realizzate per tutti gli americani. Questa non era certamente un'idea radicale, ma forse un tantino controversa in quell'ambiente particolare. Chiesero alla scrittrice Maya Angelou ${ }^{30}$ di leggere il mio testo per il filmato. Dopo riscrissi la presentazione di Lincoln, scegliendo pezzi diversi dai suoi discorsi che mettevano in luce la lotta contro la schiavitù come tema centrale del suo periodo e della sua carriera. Ovviamente Disneyland non è il luogo per presentazioni storiche sofisticate, ma credo che gli storici debbano intervenire laddove la storia è presentata a un grande pubblico per assicurare che le rappresentazioni siano adeguate. Milioni di persone hanno visto le presentazioni da me revisionate e acquisito così una comprensione storica certamente più accurata della precedente.

\footnotetext{
30 Della poetessa e scrittrice afroamericana, attivista del movimento dei diritti civili, cfr. Il canto del silenzio (Frassinelli, Milano 1996) e Unitevi nel mio nome (Frassinelli, Milano 1999).
} 
Frezza. A proposito di temi controversi, non abbiamo ancora affrontato quello della Ricostruzione. Puoi parlarci di come sei arrivato a scrivere un libro che ha cambiato la prospettiva storica su quel periodo?

Foner. Il libro sulla Ricostruzione (1988) è stato scritto nell'arco di dieci anni, non si trattava di una tesi di dottorato. Sono stato fortunato a quell'epoca, sto parlando dei tardi anni ' 70 e inizio ' 80 . La Ricostruzione era al centro di molti studi di storia. Vi era un'intera generazione di storici che stava ripensando la storia di quel periodo. Fino agli anni '70, con qualche eccezione come W.E.B. Du Bois, la prospettiva più comune sulla Ricostruzione era dominata da questa profonda visione razzista: dare dei diritti agli afroamericani era stato un gravissimo errore e aveva portato al malgoverno e alla corruzione nel Sud, mentre gli eroi erano coloro che avevano difeso la supremazia della razza bianca. Questa visione forniva la giustificazione teorica per il sistema segregazionista e le leggi $\mathrm{Jim} \mathrm{Crow}^{31}$, nonché per quelle che toglievano il diritto di voto ai neri, come succedeva in tutto il Sud fin da quel momento. Una volta che si affermò il movimento per i diritti civili, tutto quell'edificio crollò e gli storici non pensarono più che la popolazione nera fosse inferiore e non dovesse far parte della società e del governo americano.

Una volta che il vecchio punto di vista era crollato ci si domandò cosa fosse successo realmente nel periodo della Ricostruzione. Così uscirono un libro sui legislatori neri in Georgia, un altro sul passaggio tra lo schiavismo e il lavoro libero nella regione di Augusta, un altro sulla valle del Mississippi. Si pubblicavano tutti questi lavori di storia locale e inoltre volumi sui dibattiti al Congresso sulla Ricostruzione. In un certo senso sono stato fortunato che vi fosse tutto questo fermento, poiché si trattava di qualcosa di simile a quanto era successo con la schiavitù dieci anni prima. Per altro ho fatto una ricerca enorme per quel libro, negli archivi del Nord e del Sud perché volevo narrare quella storia in un modo completamente differente da quella corrente. I diversi spezzoni si venivano unificando come narrazione generale di quello che ritengo essere un periodo di cruciale importanza della storia americana. Allora si sviluppò il concetto dei diritti civili, si posero le fondamenta costituzionali della cittadinanza e per la prima volta gli afroamericani esercitarono un potere politico significativo negli Stati Uniti. Quel periodo fa nascere domande sul rapporto tra democrazia politica e democrazia economica. E naturalmente quel progetto fallì lasciando il campo ad una lunga stagione di segregazione e di leggi Jim Crow. È stato lo storico Van Woodward ${ }^{32}$ che ha definito il pe-

${ }^{31}$ Per "leggi Jim Crow" si intende quel sistema di leggi locali e dei singoli stati nel periodo 1876-1965 che imponevano un regime di segregazione razziale in tutti i servizi pubblici, poi legittimato dalla sentenza della Corte Suprema del 1896 Plessy v. Ferguson.

32 Tra le sue opere ricordiamo Origins of the New South, 1877-1913 (Lousiana State UP, Baton Rouge 1951), Reunion and Reaction: The Compromise of 1877 and the End of Reconstruction (Little, Brown and Co, Boston 1951, nuova ed. 1991), The Strange Career of Jim Crow (Oxford UP, New York 1955, nuova ed. 1974), i saggi American Counterpoint (Little Brown, Boston 1971). 
riodo dei diritti civili «la seconda Ricostruzione». Per capire quest'ultima è necessario tornare a quella precedente. Mi sento molto gratificato dal fatto che il libro sia diventato quello che propone attualmente la visione "standard" su quel periodo. Vi sono state obiezioni e critiche su questo o quel punto, vi sono storici che stanno producendo ulteriori lavori ma nessuno da allora ha provato a scrivere una storia generale della Ricostruzione. Qualcuno lo farà un giorno o l'altro.

Ciò che volevo avvenisse è davvero successo, almeno nel mondo accademico: chiunque sia interessato al tema dei diritti civili e al ruolo della popolazione afroamericana in America deve riferirsi al mio libro. L'altra sera ero andato alla cena per il premio Bancroft ${ }^{33}$ e uno dei premi è andato a un libro eccellente sul movimento dei diritti civili ad Atlanta, in Georgia, dagli anni '40 agli anni '70. Non conoscevo l'autrice, è venuta da me e mi ha detto: «Non avrei potuto scrivere il mio libro senza il tuo sulla Ricostruzione». Nel suo libro non vi è nemmeno un riferimento alla Ricostruzione, tuttavia il mio lavoro le ha fornito una cornice all'interno della quale impostare l'intero problema dei diritti civili e per questo mi sono preso il mio tempo per scriverlo. Il mio editore continuava a tallonarmi chiedendomi: «Quand'è che finirai questo libro?» Io gli rispondevo: «Voglio essere assolutamente inattaccabile, per così dire. Voglio che il lavoro abbia delle basi solide nella ricerca perché propone una visione completamente nuova». Quando ero ragazzo mio padre, in base alla sua esperienza da blacklisted, con tutte le conseguenze, mi diceva: «Qualsiasi cosa tu faccia se continui a mantenere queste idee di sinistra devi essere doppiamente bravo in modo da essere al di sopra di ogni possibile critica».

Frezza. Quando il tuo libro uscì suscitò molta discussione.

Foner. Vi furono dibattiti, discussioni, ma venne fuori in un momento favorevole per cui fu molto apprezzato. Gli unici ad essere veramente critici furono i cosiddetti "neo-confederati" 34 , che sono ancora rinchiusi in una visione razzista di quel periodo. Il libro fornì un usable past per il periodo successivo, quello dei diritti civili. La Ricostruzione era stata un periodo nel quale, pur con tutti i limiti, gli afroamericani furono integrati in molti aspetti della vita americana come non era mai avvenuto.

Frezza. Tornando un momento indietro, hai parlato di un dibattito sul tema della schiavitù. Ci puoi spiegare meglio?

33 Il riferimento di Foner è al volume di T. Brown-Nagin, University of Virginia Law School, Courage to Dissent: Atlanta and the Long History of the Civil Rights Movement, Oxford UP, Oxford 2011.

${ }^{34} \mathrm{Si}$ tratta di gruppi ultra conservatori attivi in alcuni stati del Sud nostalgici della cultura schiavista, della "causa perduta" del Sud nella Guerra civile e dei simboli ad essa legati quali la bandiera confederata. Spezzoni di questo movimento sono confluiti in tempi recenti nel Tea-Party e promuovono la «guerra teologica» per una «nazione cristiana». 
Foner. Non penso a un grande dibattito. Quello che voglio dire è che negli anni '60-'70 gran parte della storiografia importante della storia americana discuteva sul tema della schiavitù. Da Eugene Genovese a Edmund Morgan ${ }^{35}$ a David Brion Davis ${ }^{36}$, i libri più importanti di quel periodo se ne occupavano in un modo o nell'altro. Guardate ad esempio ai Premi Bancroft degli anni '70. Ma il tema non è scomparso. Gli storici scrivono ancora sulla schiavitù nonostante i risultati raggiunti dagli studi sull'argomento. Ma gli studiosi si spostarono allora sul periodo successivo chiedendosi: «Cosa successe dopo?».

Frezza. Il tema della schiavitù modificò anche la prospettiva sulla Guerra civile, sostenendo l'idea che la schiavitù ne era stata un elemento cruciale.

Foner. Questo è di estrema importanza. Se si pone la schiavitù al centro, questo fatto cambia il punto di vista sulla Guerra civile, rende l'emancipazione degli schiavi il risultato cruciale della guerra e prospetta una visione differente sul periodo della Ricostruzione. La Ricostruzione perciò diventa non più semplicemente un periodo di storia politica ma un processo attraverso il quale il paese cerca di fare i conti con la fine della schiavitù. Perciò la relazione è con il periodo precedente.

Frezza. Ritieni che oggi nell'opinione pubblica l'idea che la schiavitù sia stata un elemento centrale nella Guerra civile sia comunemente accettata?

Foner. La questione è controversa. Ciò di cui sono stato maggiormente gratificato in un certo senso è che nel sentire comune la nozione che la Ricostruzione sia stata un fatto positivo e un momento di importanti conquiste per gli afroamericani - e di conseguenza abbia dato luogo ad una reazione razzista - è abbastanza diffusa. Quando si fa riferimento alla Ricostruzione anche non esplicitamente, in special modo con Obama, si dice: «il primo senatore afroamericano dall'epoca della Ricostruzione». Si parla di quel periodo come di un passo in avanti del paese e non di un periodo di malgoverno e di corruzione.

Lorini. Potresti dire che il tuo libro sulla Ricostruzione nasce da Free Labor Free Soil Free Men? Penso che vi sia una continuità tra questi due lavori.

Foner. Vi è una continuità perché la nozione di lavoro libero è centrale in entrambi. Nel primo libro scrivo a proposito dell'abolizione della schiavitù in

35 Di cui si veda American Slavery, American Freedom. The Ordeal of Colonial Virginia (1975) Norton, New York 2003.

36 Tra le sue opere: The Problem of Slavery in Western Culture (Cornell UP, Ithaca-New York 1966), The Problem of Slavery in the Age of Revolution, 1770-1823 (Cornell UP, IthacaNew York 1975), Slavery and Human Progress (Oxford UP, Oxford 1984), Inhuman Bondage: The Rise and Fall of Slavery in the New World (Oxford UP, Oxford 2006). 
base all'idea di lavoro libero, non partendo da un punto di vista morale o politico ma dalla concezione di cosa dovrebbe essere una nazione fondata sul lavoro libero. Il volume sulla Ricostruzione tratta di come gli uomini politici di allora tentarono di rafforzare l'idea di lavoro libero e di come questo tentativo non funzionò. La nozione di lavoro libero era in un certo senso inadeguata a trattare la situazione del Sud dopo la fine della schiavitù e finì per spezzarsi in direzioni differenti. Da un lato si mosse verso una sorta di conflitto di classe nel Nord, dall'altro si sviluppò una nozione molto oppressiva di lavoro nel Sud. Vi è una continuità: il tema del lavoro libero ricorre in entrambi i libri.

Frezza-Lorini. Poi c'è stato il tuo libro sulla storia della libertà americana, l'unico tradotto in italiano. Puoi parlarci di questa idea di libertà che mostra molti aspetti diversi nella storia americana? Quel libro è stato tradotto in moltissime lingue, molti dei tuoi ex-studenti di provenienza internazionale ne volevano la traduzione in modo da poterlo usare a loro volta con $i$ loro studenti. Cosa aggiungeresti a quel libro se dovessi scriverlo oggi?

Foner. Anche il libro sulla storia della libertà americana aveva lo scopo di fornire uno usable past per coloro che intendevano la libertà in modo diverso rispetto a quella che ancora oggi è dominante: vale a dire l'idea di libertà del periodo di Reagan, di «togliersi il governo di dosso», di eliminare dal mercato ogni forma di regolamentazione, nella totale assenza di qualsiasi forma di coesione e di responsabilità sociale. Anche oggi, se dovessi riscrivere quel libro, farei riferimento ad altre definizioni di libertà emerse nella nostra storia, sottolineando come questa parola, "libertà", centrale nel vocabolario politico americano, sia stata sempre un'idea contestata, così come la definizione di chi abbia il diritto di godere di questa libertà americana. È vero, il libro ha avuto un'ampia circolazione negli Stati Uniti e all'estero. Mi è stato detto che la traduzione cinese ha avuto un grande impatto sugli intellettuali di quel paese che sono alla ricerca di idee sulla libertà diverse da quelle dei gruppi dirigenti.

Lorini. Ora passiamo al tuo ultimo lavoro, quello su Lincoln ${ }^{37}$. Hai detto che è meno di una biografia intellettuale ma anche più di questa perché in realtà hai ricostruito tutte le ideologie politiche dell'epoca, l'ambiente politico. Sono rimasta molto colpita dalla tua idea della "crescita" di Lincoln. Puoi dirci qualcosa su questo, in quanto penso ad altri leader importanti che forse sotto una pressione come quella sperimentata da Lincoln non sono in effetti cresciuti. Penso ad Obama...

37 E. Foner, The Fiery Trial. Abraham Lincoln and American Slavery, Norton, New York 2010, vincitore nel 2011 dei premi Bancroft, Lincoln e Pulitzer per la storia. 
Foner. Obama probabilmente osserverebbe: «Guarda, Lincoln ha avuto gli abolizionisti e i repubblicani che lo spingevano». Chi sta spingendo Obama nella direzione giusta? Non sto difendendo Obama, sto soltanto dicendo che Obama conosce la storia e probabilmente direbbe: «Se avessi la pressione del movimento abolizionista anch'io crescerei». Il mio libro vuole sottolineare due problemi. Il primo è una questione filosofica che risale a Tucidide: una cosa è l'individuo e una il suo tempo; è l'uomo che fa la storia o è la storia che fa l'uomo? Ovviamente sono vere ambedue le cose. Lo stesso Lincoln dice: «Io non controllavo gli avvenimenti, questi ultimi controllavano me». Tuttavia devi avere il desiderio di essere controllato. Molte persone affogano in una crisi mentre Lincoln cresce nella crisi, perciò ci deve essere un aspetto della sua personalità che gli permette di farlo. Guardate il suo successore Andrew Johnson che fu presidente in un momento di grande crisi e affonda, non riesce in nessun modo a cogliere le opportunità offerte dalla crisi. Semplicemente non ha le qualità necessarie quali che esse siano. L'altro problema è il rapporto tra leadership politica e movimenti sociali. Questo è ciò che veramente mi interessa analizzare nel libro. Perché ho scritto un libro su Lincoln? Vi sono decine di migliaia di testi su Lincoln e io non ho mai scritto su di lui; è presente in molti dei miei libri come una comparsa. Nel lavoro sulla Ricostruzione, all'inizio sono molto critico su Lincoln. Molti ritengono che questo libro gli sia più favorevole perché la sua carriera si sviluppa verso, credo, la strada giusta. In realtà nella prima parte sono estremamente critico per alcune sue idee sul razzismo e la schiavitù. Il problema maggiore che si incontra a scrivere un libro su Lincoln è che esiste una letteratura talmente vasta che, nella maggior parte, lo pone su una sorta di piedistallo: Lincoln è un grande uomo, è un eroe, è colui che promuove l'emancipazione. Naturalmente quando fai questo tipo di operazione qualcun altro arriva con un grosso piccone e lo butta giù. Vi sono libri che dicono che era un razzista, che non si interessava affatto della schiavitù, che era solo un politico che cercava di fare carriera.

Frezza. Lincoln faceva parte di un'associazione che voleva cacciare tutti gli afroamericani dal paese.

Foner. Esattamente. Il mio libro mette in evidenza questo, più di altri. Ma Lincoln si evolve rispetto a quella posizione. Uno studioso di Lincoln una volta mi disse: «Mi piace molto il tuo libro perché ora sei dalla parte di Lincoln» e io pensai: «Questo è assurdo!» Io non mi considero né dalla sua parte né contro. Vi è una specie di industria che cresce su Lincoln, un po' come sui padri fondatori, ma molto di più, e la maggior parte della produzione non è dovuta a storici professionisti ma ad avvocati, filosofi, politici, giornalisti. Alcuni probabilmente sono anche buoni libri, ma gli autori non sono interessati ai problemi che interessano gli storici, ma piuttosto ad aspetti secondari del suo carattere. 
Frezza. Hai detto che eri interessato al rapporto tra movimenti sociali e leadership.

Foner. Sì e questo rimanda al modo in cui io scrivo i miei libri. Molti nascono da un senso di malessere per quella che è l'opinione corrente. Quello sulla Ricostruzione voleva distruggere una vecchia prospettiva che mi dava molto fastidio. Il mio volume sulla libertà era motivato dal rifiuto di quella che ritenevo fosse un'appropriazione di questo concetto da parte della destra, sotto Reagan: l'unico modo nel quale si poteva parlare di libertà riguardava la diminuzione delle prerogative del governo. Volevo mostrare che vi era un intero apparato di concetti sulla libertà completamente differente nella nostra storia e che quello corrente non era affatto l'unico. Il mio libro su Lincoln è in parte motivato dal malessere suscitatomi dalla letteratura che negli ultimi dieci anni lo contrappone agli abolizionisti. Lincoln viene visto come il leader moderato, ragionevole e pragmatico. Molti mi hanno criticato dicendo che la mia posizione è assurda, che gli abolizionisti non sono dei politici, che non capiscono quanto sta succedendo, che sono dei fanatici che sostengono delle idee folli. Ora questo è assurdo.

Lorini. Vi erano molte tipologie di abolizionisti...

Foner. Certamente, e tra l'altro gli abolizionisti avevano idee politiche molto chiare. Questo mostra un fraintendimento della politica. La politica non significa soltanto il processo elettorale. Gli abolizionisti sono politici che cercano di modificare il comune sentire. Volevo uscire dalla retorica del «buon Lincoln»e dei «cattivi abolizionisti», che produceva un fraintendimento di ambedue le posizioni. Lincoln non è un abolizionista, non ha mai detto di esserlo e io non cerco di farlo diventare tale. Vi sono libri che sostengono che segretamente era un abolizionista e che non poteva dirlo apertamente. Non è vero. Vi erano certamente grandi tensioni tra gli abolizionisti e Lincoln ma egli capisce che è sulla stessa barca; stanno muovendosi nella stessa direzione con metodi differenti e con velocità diverse. Lincoln capisce che il movimento abolizionista rende possibile una politica contro la schiavitù. Senza gli abolizionisti non puoi avere Lincoln. Egli fa cose differenti; è un politico, lavora all'interno delle istituzioni. William Lloyd Garrison ${ }^{38}$ brucia la Costituzione, gli abolizionisti sono contro il potere e Lincoln è all'interno del sistema. Ma lui capisce che deve trattare con loro, parlare con loro, criticarli, così come loro criticano lui. A un certo punto durante la Guerra civile Lincoln, parlando di questo gruppo di radicali, disse: «sono diavoli, ma sono diavoli che affrontano Zion». In altre parole, «mi disturbano, ma miria-

38 William Lloyd Garrison fondò nel 1831 il giornale abolizionista «The Liberator» per promuovere l'immediata e totale emancipazione di tutti gli schiavi. Nel 1844 Garrison bruciò pubblicamente una copia della Costituzione. 
mo allo stesso obiettivo». Non cerco di glorificare gli abolizionisti, fecero degli sbagli anche loro. Credo che sia questa intersezione tra movimenti sociali e leader politici che davvero mi interessa e ritengo che questa posizione riporti Lincoln all'interno del suo tempo. Molto spesso i libri su di lui lo pongono fuori dal suo contesto in modo tale da farlo diventare auto-referenziale. Se vuoi sapere perché Lincoln fece alcune cose come presidente devi studiare la sua precedente carriera di avvocato.

Lorini. Trovo parte di questi temi trattati nel tuo libro su Lincoln in continuità con l'analisi del rapporto tra il radicale Tom Paine e i politici dell'America rivoluzionaria: una realtà sociale molto vivace nella Filadelfia di quel periodo.

Foner. Occorre collocare le persone nel loro contesto. Io cerco di collocare Lincoln nell'Illinois di allora. Quando si leggono le sue opere con attenzione si trovano cose alle quali non si era pensato prima. È un grande maestro della lingua inglese, uno scrittore molto attento e al tempo stesso brillante. Si deve leggerlo con calma. Come sia diventato tale non ho idea, ha fatto un solo anno di scuola, era completamente autodidatta. Vi è un momento nel 1856, nella parte settentrionale dell'Illinois molto contraria alla schiavitù, nel quale il Partito repubblicano locale aveva nominato un abolizionista al Congresso come candidato; alcuni repubblicani più conservatori avevano detto che non lo volevano e nominarono un altro candidato che avrebbe così diviso il voto, e in effetti i democratici vinsero. Molti di questi conservatori erano amici di Lincoln perché egli apparteneva all'ala più conservatrice del Partito repubblicano nell'Illinois. Egli si recò al distretto, parlò con il pubblico e poi scrisse a un amico: «Sai, io credo che voi dobbiate veramente ritirare questo candidato perché prima di tutto non potete dividere il voto. Questo sarebbe assurdo per un partito politico. Io ho anche parlato con il pubblico, io non sono interamente d'accordo con loro, ma sono colpito dal loro entusiasmo». In pratica diceva: «Abbiamo bisogno di loro, fanno parte del Partito repubblicano». Sono la base, per usare un termine moderno, e non si vince solo con la propria base ma non si può buttarla fuori dal partito.

Lorini. Questo è anche un messaggio importante per il nostro pubblico italiano.

Foner. È una lezione per Obama. Mi piacerebbe che leggesse questo passo. Obama non ha un interesse particolare per quella che io chiamo la base quella più liberale, i democratici di sinistra, perché li dà per scontati. Certo, votano per lui piuttosto che per Romney ma ha bisogno di qualcosa di più di un voto, ha bisogno del loro entusiasmo, come aveva detto Lincoln che era un politico molto accorto. 
Lorini. Per questo motivo Lincoln è cresciuto come leader politico, a differenza di Obama.

Foner. Forse nel secondo mandato crescerà anche lui. Ma Lincoln vive nel momento della più grande crisi della storia americana, perciò egli cresce sotto l'impatto degli eventi; alla fine della guerra parla a proposito della razza in un modo molto significativo per quell'epoca, per un uomo che aveva un punto di vista razzista nel periodo precedente. Come ho osservato nel libro, due cose lo hanno colpito in particolare: una è il servizio militare dei soldati afroamericani - più di 200.000. Lincoln si rende conto che combattendo per la nazione stanno diventando cittadini. Non si può dire loro «andate via dal paese ora». L'altra riguarda i contatti personali. Egli incontrò Frederick Douglass e altri leader afroamericani. Non conosceva nessun nero prima della guerra. Incontrò persone di colore che erano molto sofisticate dal punto di vista politico, che erano attive, intelligenti quanto lui. Persone che potevano sostenere qualsiasi discorso sul mondo politico e ritengo che anche questo contribuì ad ampliare il suo punto di vista sulle capacità degli afroamericani.

Frezza. Pensando oggi a Obama, quali potrebbero essere le somiglianze e le differenze?

Foner. Siamo in un mondo diverso. Io ho contrastato Obama per non essere aperto alle critiche. Lincoln era incredibilmente aperto, non si preoccupava se vi erano persone che andavano alla Casa Bianca e gli dicevano che aveva torto. Questo è il motivo per cui Frederick Douglass lo andò a trovare, non per dargli delle pacche sulle spalle, ma per dirgli: «Guarda Lincoln devi proteggere questi soldati afroamericani quando vengono catturati dai Confederati, devi dar loro la stessa paga che dai ai soldati bianchi». Lincoln non se ne ebbe a male, aveva questo tipo di umiltà. Obama credo abbia un carattere diverso e si circonda di yes-men. Non vi è nessuno nella cerchia ristretta dell'amministrazione che gli offra un punto di vista alternativo.

Frezza. Penso alla crisi del 1929 e a F.D. Roosevelt. Non soltanto egli accettava le critiche ma cercava anche di mettere insieme persone con posizioni diverse in modo che trovassero una soluzione comune.

Foner. Assolutamente! Quando A. Philip Randolph ${ }^{39}$ andò da Roosevelt e gli disse: «devi emanare un ordine che proibisca la discriminazione razziale nelle industrie di guerra», Roosevelt gli rispose: «Fate in modo che io possa farlo», cioè andate nelle strade e spingetemi a farlo. Si trattò della prima mar-

${ }^{39}$ A. Philip Randolph, leader afroamericano, guidò la Brotherhood of Sleeping Car Porters, sindacato di lavoratori prevalentemente neri e la marcia su Washington, spingendo il presidente Roosevelt ad emettere l'Executive Order 8802 che bandiva la discriminazione razziale nelle industrie belliche. 
cia a Washington nel 1941 e il Congresso si spaventò talmente all'idea di migliaia di afroamericani che marciavano a Washington che, quando Roosevelt emanò l'ordine, tutti dissero: «Bene». Questo non è il modo di procedere di Obama. Obama non direbbe: «fate in modo che possa farlo», Obama dice: «Abbiate fiducia in me». Io ero entusiasta di Obama, ma dopo la sua elezione ha nominato persone responsabili della crisi come suoi consiglieri economici. In una conferenza stampa la gente chiedeva: «Dove sta il cambiamento? Tu hai fatto la tua campagna elettorale su questo». E Obama rispondeva: «Io sono il cambiamento». Allora io osservai: «Qui c'è quello che Kruschev chiamava il "culto della personalità"!». Lincoln non ha mai detto: «Io sono il cambiamento». Egli diceva: «Vi è un intero universo di cambiamenti là fuori», mentre Obama ha questa idea: «io sono il cambiamento» e nessuno ha il diritto di suggerirgli nulla. Forse in un secondo mandato avrà maggiore umiltà. 
Copyright $(\odot$ FrancoAngeli

N.B: Copia ad uso personale. È vietata la riproduzione (totale o parziale) dell'opera con qualsiasi mezzo effettuata e la sua messa a disposizione di terzi, sia in forma gratuita sia a pagamento. 


\title{
“Spara e prega!". Il cinema israeliano a trent'anni dalla guerra del Libano
}

\author{
Marcella Simoni
}

\begin{abstract}
Israeli cinema on Lebanon war. The first decade of the new century has seen the appearance of numerous Israeli films by different directors, and at least one fiction book, who addressed various aspects of the Israeli involvement in Lebanon in some of its phases: the 1982 Lebanon War, the occupation of Beaufort and the evacuation from the so-called Security Zone (2000). Using these movies and the book as a sources and framing them in the critical literature on the subject - this article discusses if such a cultural production gave rise to a public or political debate in Israel, or if they just responded to the individual needs of the directors who filmed them.
\end{abstract}

Key words: Israeli cinema, trauma, testimony, Lebanon

Parole chiave: cinema israeliano, trauma, testimone, Libano

Questo articolo prende in esame quattro lungometraggi israeliani usciti tra il 2007 e il 2009, che affrontano il periodo iniziale e conclusivo della presenza israeliana in Libano, dal 1982 al 2000: Valzer con Bashir (Ari Folman, 2008), Lebanon (Samuel Maoz, 2009), Beaufort (Joseph Cedar, 2007) e Wasted (Nurit Kedar, 2007). Valzer con Bashir ha portato sullo schermo l'ingresso dell'esercito israeliano (Idf) in Libano, l'assedio di Beirut ovest e il massacro dei campi profughi palestinesi di Sabra e Shatila (16-18 settembre 1982) facendo uso di una particolare tecnica di animazione ${ }^{1}$. Lebanon narra le storie di un gruppo di carristi alla prima prova del fuoco durante quella stessa guerra. Tratto dal romanzo Tredici soldati di Ron Leshem ${ }^{2}$, il film Beaufort si concentra sulle vicende degli ultimi occupanti dell'omonima fortezza crociata, conquistata il primo giorno di guerra e occupata per i successivi 18 anni, simbolo dell'ultimo periodo dell'occupazione della Zona di Si-

\footnotetext{
${ }^{1}$ La tecnica utilizzata è una variazione del Cgi (computer generated imagery) animation: $\mathrm{i}$ modelli per le animazioni provengono dai movimenti videoregistrati degli attori reali che sono poi suddivisi e calibrati con un software e infine ricomposti in forma disegnata e animata, come in un puzzle.

2 R. Leshem, Tredici soldati, Rizzoli, Milano 2007.

«Passato e presente», a. XXXI (2013), n. 88
} 
curezza da parte dell'Idf, che è stata poi evacuata il 24 maggio $2000^{3}$. Un secondo film documentario, Wasted, è partito dallo stesso romanzo per portare sullo schermo i volti e le testimonianze reali dei soldati che erano stati gli ultimi occupanti di quel forte.

Si pensava che sarebbero state le ultime voci di Israele in Libano ${ }^{4}$ ma, mentre questi film erano in lavorazione, la Seconda guerra del Libano (12 luglio-14 agosto 2006) ha aperto un nuovo atto di questo confronto, che ha inevitabilmente trasformato e influenzato la percezione del pubblico e il giudizio delle giurie su questi film nei vari festival internazionali in cui hanno generalmente trionfato ${ }^{5}$. Di impronta anti-militarista, hanno ottenuto un enorme successo per motivi legati alla cinematografia - tra cui le scelte estetiche dei registi, le tecniche di regia e la modalità narrativa - e alla politica: dietro l'atteggiamento polemico nei confronti del governo Begin (1981-83) che aveva dichiarato la guerra del 1982 si leggeva infatti anche una presa di posizione contro il governo Olmert (2006-09), che aveva ordinato la campagna dell'estate 2006.

Non è la prima volta che la guerra del Libano compare sugli schermi cinematografici in Israele. Nel decennio 1982-1992 erano usciti quattro film su questo argomento, che avevano avuto una circolazione internazionale molto limitata: Ricochets (Eli Cohen, 1982), Burning Memory (Yossi Somer, 1989), A Season for Cherries (Haim Bouzaglo, 1991) e Cup's Final (Eran Riklis, 1992). Con l'eccezione di Ricochets - prodotto dallo stesso Idf - si trattava di film blandamente antimilitaristi, in cui il soldato israeliano era ritratto seguendo l'estetica consumista degli anni '80. In A Season for Cherries il protagonista è un pubblicitario dal sorriso seducente che prende la vita con filosofia e invita a divertirsi, salvo poi morire attraversando un ponte minato, mentre in Cup's Final il protagonista parte per il fronte sperando che la guerra finisca prima dei mondiali di calcio di Spagna, avendo già acquistato i biglietti per tutte le partite. Si tratta di soldati non giovanissimi, generalmente riservisti, li-

\footnotetext{
${ }^{3}$ L'istituzione di una Zona di Sicurezza sotto la responsabilità congiunta di forze israeliane e libanesi come deterrente per attacchi contro il nord di Israele e per impedire infiltrazioni palestinesi in Israele faceva parte di un accordo del 1983 tra Israele e Libano, che tuttavia il Libano non ha mai ratificato. Nonostante il ritiro dal Libano del 1985, l'Idf lasciò un piccolo contingente nella Zona di Sicurezza, un territorio dai confini e dalle dimensioni variabili a seconda del momento storico (dai 5 ai $25 \mathrm{~km}$, compresi tra il confine settentrionale di Israele e le alture del Golan). Il ritiro completo dell'Idf dal Libano è avvenuto nel 2000 con il governo di Ehud Barak, che ha così implementato la risoluzione Onu n. 425 (19 marzo 1978) e ha quindi portato alla conclusione definitiva della guerra iniziata nel 1982. Per una discussione del ritiro israeliano dalla zona di sicurezza cfr. A. Selah, Civil Society, the Military and National Security: The Case of Israel's Security Zone in South Lebanon, «Israel Studies», 12 (2007), n. 1, pp. 53-78.

${ }^{4} \mathrm{Cfr}$. invece la memoria di guerra di A. Isaacs, Lebanon II, «Common Knowledge», 15 (2009), n. 1, pp. 97-153.

5 Per una lista dei riconoscimenti attribuiti a Valzer con Bashir, a Lebanon e a Beaufort cfr. waltzwithbashir.com/home.html, www.imdb.com/title/tt1483831/awards e www.imdb.com/ title/tt0758732/awards, accesso del 2 febbraio 2012.
} 
berali se non di sinistra, che si ritrovano in Libano loro malgrado, e che a tratti provano a identificarsi con la sofferenza palestinese, senza troppa convinzione ${ }^{6}$. Per citare Raz Yosef, critico cinematografico e docente di cinema studies all'Università di Tel Aviv, il soldato di questi film «spara e piange»7.

Si tratta, in generale, di ruoli costruiti in maniera meno complessa rispetto a quelli che ritroviamo nei film del decennio successivo, tra cui appunto Valzer con Bashir e Lebanon, ben più vicini alla biografia dei registi e degli attori che vi hanno preso parte. Vi è un'altra differenza importante tra questo primo gruppo di film sul Libano usciti negli anni ' 80 e ' 90 e quelli del decennio successivo: nei primi è ben presente il nemico palestinese, seppur rappresentato in maniera stereotipata, nei panni del guerrigliero crudele $\mathrm{o}$ dell'intellettuale che aveva studiato in Italia negli anni '70 (Cup's Final), o attraverso l'immagine metaforica di una mano che mette cariche esplosive sotto i ponti, nei mercati o dentro un nido di colombe bianche (A Season for Cherries); nei secondi la rappresentazione del nemico è assente.

L'"altro" è assente anche da queste pagine, che pongono l'accento sul coté israeliano e che discutono come il cinema israeliano abbia rappresentato, a trent'anni di distanza, situazioni, luoghi, soldati, e vicende dell'esperienza libanese in due dei suoi momenti principali: il 1982 e il 2000. Questo articolo non affronta quindi le conseguenze della guerra del 1982 sulla popolazione palestinese e libanese, il suo impatto sulle diverse zone o gruppi religiosi del paese, il rapporto tra questa guerra e l'ascesa della comunità sciita, né quale sia stato il ruolo della Siria o degli Stati Uniti. Sono temi che sono stati ampiamente trattati dalla storiografia, al pari del tragico intreccio tra guerra civile libanese (1975-1990) e la parte del conflitto israelo-palestinese che è stata combattuta in Libano, a partire dall'operazione Litani (1978) ${ }^{8}$. Limitandomi quindi ad alcuni aspetti della rappresentazione della guerra del Libano, nella prima parte riassumo alcuni aspetti che l'hanno caratterizzata, tra cui la ben nota mancanza di consenso interno. Nella seconda considero come viene ricordata attraverso le testimonianze di alcuni soldati. Nella terza parte metto a

${ }^{6}$ N. Gertz, The Medium that Mistook Itself for War: Cherry Season in Comparison with Ricochets and Cup Final, «Israel Studies», 4 (1999), n. 1, pp. 153-74.

${ }^{7} \mathrm{R}$. Yosef, The Politics of Loss and Trauma in Israeli Cinema, Routledge, New York-London 2011, p. 25.

${ }^{8}$ Cfr. W. Khalidi, Conflict and Violence in Lebanon, Harvard UP, Boston 1979; R.I. Khalidi, Lebanon in the Context of Regional Politics: Palestinian and Syrian Involvement in the Lebanese Crisis, «Third World Quarterly», 7 (1985), n. 3, pp. 495-514; R. Fisk, Pity the Nation. Lebanon at War, Oxford UP, Oxford 1990. Cfr. anche S. Kassir, Beirut. Storia di una città, Einaudi, Torino 2009 (ed. or. Librairie Arthème Fayard, Paris, 2003, trad. it. M. Marchetti); M. Simoni (a cura di), Il Libano prima e dopo il 1982, «Passato e presente», 87 (2012), n. 3 , pp. 19-37; E. Chiti (a cura di), Frammenti di storia, società, cultura, Mesogea, Messina 2012. Per un'analisi dell'elaborazione della memoria collettiva della guerra civile e della Guerra del 1982 nel cinema libanese cfr. l'analisi di The Tornado (Samir Habchi, 1992), West Beirut (Ziad Doueiri, 1998), In the Shadows of the City (Jean Chamoun, 2000) proposta da N. Hourani, The Militiaman Icon. Cinema, Memory and the Lebanese Civil Wars, «CR: The New Centennial Review», 8 (2008), n. 2, pp. 287-307. 
confronto la rappresentazione dell'uscita dal Libano - la cui metafora principale è stata l'evacuazione dalla fortezza di Beaufort - nella sua versione letteraria (Tredici soldati), cinematografica (Beaufort) e documentaria (Wasted).

\section{Libano 1982. La nascita dell'anti-eroe oltre la letteratura}

La figura dell'anti-eroe in ambito militare non è nuova nella letteratura e cultura israeliana, a cominciare da due celebri racconti: L'ultimo comandante di Abraham B. Yehoshua pubblicato nel 1960 e La via del vento di Amos Oz scritto nel $1962^{9}$; essa è tuttavia comparsa nel cinema a partire dalla fine degli anni '90, forse in linea con l'idea che, nel nuovo secolo, sia il cinema a rappresentare la nuova coscienza critica del paese ${ }^{10}$. Già in Kippur (2000) un film con ampi riferimenti autobiografici all'esperienza del regista Amos Gitai durante la Guerra del 1973 - dominavano la confusione e la difficoltà nell'organizzazione delle operazioni militari e/o di salvataggio; la macchina da presa non puntava sulla linea del fronte ma sulle retrovie, dove i soldati dovevano gestire l'evacuazione dei morti e dei feriti, in una rappresentazione dell'Idf molto diversa da quella stereotipata e glorificante che compariva in Tsahal diretto da Claude Lanzmann nel 1994. In Infiltration (Dover Koshashvili, 2009) - tratto dall'omonimo (ma ben più radicale) romanzo di Yehoshua Kenaz del 1986 - il mito dell'Idf veniva decostruito con riferimento al suo periodo formativo, gli anni '50, quando Moshe Dayan era capo di Stato maggiore e l'eroe della Guerra del Sinai (1956). In Infliltration ci troviamo di fronte a un gruppo di reclute disturbate, troppo deboli per sostenere un qualsiasi addestramento, di retroterra geografici e culturali troppo diversi per potersi davvero integrare, o eccessivamente motivate per riuscire a sostenere l'umiliazione fisica e morale continuamente inflitta loro da superiori sadici.

A questa graduale decostruzione dell'immagine di una delle istituzioni storicamente più condivise all'interno della società israeliana hanno in parte contribuito la letteratura e il cinema su un piano culturale; dal punto di vista storico-politico tuttavia, è stata determinante la combinazione di fattori sociali, politici e militari che è emersa durante la guerra del 1982.

Come è noto, la guerra del Libano è stata la prima che l'Idf ha combattuto senza il sostegno quasi unanime dell'opinione pubblica, e anche la prima non veramente difensiva condotta dal governo di Menachem Begin (Likud). Fuori da Israele, la guerra del 1982 ha visto un forte movimento di opinione pubblica ebraica e non, che ha preso le distanze dal governo israeliano per aver scelto l'opzione militare; basti ricordare la presa di posizione di Primo Levi e di altri e la fondazione e la lunga mobilitazione del Comité Palestine-Israël

9 A.B. Yehoshua, Tutti i racconti, Einaudi, Torino 1999; A. Oz, Where the Jackals Howl and other stories, Vintage, London 1981.

${ }^{10}$ M.G. De Bonis - A. Schweitzer - G. Spagnoletti (a cura di), Il cinema israeliano contemporaneo, Marsilio, Venezia 2009, p. 11. 
Vivront a Parigi ${ }^{11}$. In Israele invece, la guerra del 1982 ha visto la nascita di diverse associazioni per l'obiezione di coscienza, tra cui Yesh Gvul (C'è un confine/limite), Soldiers against silence e The Committee against the war in Lebanon, tutte fondate nel 1982, a cui si aggiunse l'anno successivo Parents against silence. Ugualmente note sono le manifestazioni di massa che si svolsero in Israele a seguito dei massacri di Sabra e Shatila, e per l'istituzione di una commissione di inchiesta governativa sulle responsabilità israeliane ${ }^{12}$.

La debolezza del fronte interno e gli altri fattori appena menzionati contribuiscono a spiegare come, a circa trent'anni di distanza, la Guerra del Libano venga rappresentata non attraverso l'eroismo e il sacrificio dei soldati, o la tattica e la strategia dei comandanti (come nel caso di altre guerre e di altri film ${ }^{13}$ ) ma, al contrario, attraverso le crisi di una serie di anti-eroi, di soldati generalmente intimoriti e confusi. Una delle differenze tra questa guerra e le precedenti dal punto di vista del fronte interno è ben riassunta nelle osservazioni del protagonista di Valzer con Bashir, che torna in Israele durante la sua prima licenza:

Quando ero piccolo, diciamo quando avevo dieci anni e c'era un guerra, tutto si fermava; mentre i padri erano al fronte, tutti i bambini stavano con le madri in casa, con le finestre e le persiane chiuse e le luci spente, aspettando un aereo con una bomba che li uccidesse tutti. Nessuno si sognava neanche di uscire di casa. E ora, torno a casa per la prima volta dopo sei settimane in Libano e vedo che la vita continua come se niente fosse ${ }^{14}$.

${ }^{11}$ Sull'Italia cfr. G. Schwarz, Gli echi italiani della guerra del Libano (1982). Considerazioni su antisemitismo, autocoscienza ebraica e memoria della Shoah, «Laboratoire italien. Politique et société», 11 (2011), pp. 133-58; sulla Francia cfr. International Institute for Social History, Amsterdam (Iish), collezione Comité Palestine-Israël Vivront, b. 3, per le lettere di ebrei francesi contro l'invasione del Libano indirizzate all'ambasciata israeliana in Francia e alla stampa francese.

12 Nel 1983 la commissione di inchiesta governativa Kahan attribuì la responsabilità diretta dei massacri ai falangisti di Bashir Gemayel, ma ne assegnò la responsabilità indiretta al ministro della Difesa Ariel Sharon ritenuto responsabile, assieme al capo di Stato maggiore Rafael Eitan, di negligenza nella difesa dei cittadini di Beirut sotto controllo israeliano; il ministro degli Esteri Itzhak Shamir venne assolto. Per la traduzione autorizzata del rapporto della commissione Kahan, cfr. Israel: Final Report of the Commission of Inquiry into the Events at the Refugee Camps in Beirut, «International Legal Materials», 22 (1983), n. 3, pp. 473-520. La guerra del 1982 è da considerarsi una cesura anche dal punto di vista della modalità dell'espressione del dissenso politico in Israele; fu infatti a una manifestazione perché il governo adottasse le raccomandazioni della commissione Kahan che Yona Avrushmi, un attivista di destra, lanciò una granata tra i manifestanti, uccidendo Emil Grunzweig, insegnante, ufficiale delle riserve e attivista pacifista. A seguito di questo avvenimento Sharon si dimise da ministro degli Esteri (restando tuttavia nel governo come ministro senza portafoglio).

${ }^{13}$ N. Ben-Shaul, Il cinema israeliano sulla guerra, in M.G. De Boni-A. Schweitzer-G. Spagnoletto (a cura di), Il cinema israeliano, cit., pp. 147-65.

14 A. Folman, Waltz. With Bashir, 2007, 47:05-47:50 (così indico, qui e di seguito, i minuti del film). 
Sia in Valzer con Bashir che in Lebanon, il secondo film sul 1982, i protagonisti rientrano facilmente nella categoria degli anti-eroi: sono in stato confusionale, paralizzati dal panico, spesso perdono il controllo della situazione e di se stessi, hanno una paura che si manifesta in vario modo: attraverso lo scarso controllo che hanno sui propri corpi (piangono, tremano, urinano, vomitano, si addormentano nelle situazioni meno indicate) e che sembra rappresentare uno specchio dello scarso controllo che l'Idf aveva sul territorio, e una ironica presa di distanza dalla scritta incisa sul carro armato di Lebanon: «L'uomo è d'acciaio; il carro armato è solo un pezzo di ferro». Il puntatore di Lebanon, Shmuel (che non a caso porta lo stesso nome del regista), non è in grado di premere il grilletto, con gravi conseguenze per tutti coloro che gli sono attorno, dentro e fuori il carro armato. In entrambi i film non si può non notare il volume di fuoco che viene riversato all'ingresso in Libano. Come ha testimoniato Asher Fahan, ventiduenne all'epoca, le cui parole sono state raccolte da Irit Gal e Ilana Hammerman: «continuavamo ad avanzare riversando una quantità di fuoco enorme»15. Questa situazione trova una corrispondenza esatta in Valzer con Bashir, nella sequenza in cui Ari Folman descrive il flashback del primo giorno di guerra:

Avevo appena diciannove anni, non avevo ancora cominciato a radermi, siamo su una strada, da un lato un frutteto, dall'altra il mare e noi spariamo. Non so a chi. Semplicemente spariamo. Spariamo come matti, fino a sera spariamo.

Questa stessa situazione emerge anche da un dialogo con un compagno:

- Spara!

- A chi?

- Che ne so? Spara!

- Non sarebbe meglio pregare?

- Spara e prega!16

Da un punto di vista narrativo, sia in Valzer con Bashir che in Lebanon, i soldati israeliani sono ritratti in grave difficoltà durante la guerra e per i successivi trent'anni. Questa rappresentazione porta lo spettatore ad identificarsi con la sofferenza del soldato piuttosto che con quella delle sue vittime, un risultato che è ottenuto anche grazie alla scelta di attori particolarmente attraenti, come in Lebanon per esempio, o in Beaufort. A facilitare questa identificazione, vi è anche il fatto che la vittima/il nemico è spesso assente da queste narrazioni contemporanee: non porta un'uniforme in Lebanon e raramente lo si vede in Valzer con Bashir. Quando compare, sia nella rappresentazione cinematografica che nella testimonianza storica, si tratta di un nemico

15 I. Gal, I. Hammerman, De Beyrouth à Jénine. Témoignages des soldats israéliens sur la guerre du Liban, La Fabrique éditions, Paris 2003, p. 29.

16 Le due citazioni vengono da A. Folman, Waltz with Bashir, rispettivamente ai minuti 22:50.23:24 e 24:15-24:39. 
che non solo non indossa un'uniforme, ma che spesso si nasconde tra civili, per esempio nei panni di un ragazzino che spara con un Rpg (Rocket-propelled granade) dentro un frutteto ${ }^{17}$.

In Lebanon troviamo una delle rappresentazioni più drammatiche di questa mancanza di confini tra dimensione militare e civile: chiamato a sostegno di una pattuglia di soldati israeliani contro cui si spara dai piani alti di una casa, il carro armato entra in un cortile dove, quando il protagonista riesce infine a fare fuoco, lascia in vita solo una madre che lo spettatore vede (attraverso lo stesso mirino da cui ha sparato il protagonista) vagare nuda tra le macerie della propria casa e della propria famiglia. Come tante testimonianze di soldati tornati dal Libano ancora durante lo stesso 1982, è stata proprio questa dimensione di una guerra condotta tra i civili a far emergere l'anti-eroe reale e non solo quello letterario o cinematografico, attraverso una serie di prese di posizione personali e politiche inedite fino ad allora nella società israeliana, e attraverso la fondazione di diversi movimenti per l'obiezione di coscienza. Come ricorda Shuki Yashuv, intervistato da Irit Gal e Ilana Hammerman, la scena che lo aveva spezzato era stata una «operazione di polizia per decidere chi fosse un terrorista e chi no», in cui avevano costretto degli uomini, tra cui alcuni con «un'aria particolarmente degna», a sedersi per terra con le mani legate, una scena di «selezione di civili» («tri de civils que m'a fait craquer») ${ }^{18}$.

\section{Le testimonianze, il trauma e la memoria}

Da un punto di vista storico, alcune testimonianze di soldati che presero parte ai combattimenti riassumono le difficoltà di una generazione nel comprendere e nel giustificare le ragioni di questa guerra, combattuta attraverso centri urbani (per esempio Tiro, Sidone e Beirut) e con il coinvolgimento di un numero di civili ben più ampio di qualsiasi altra guerra combattuta dall'Idf fino ad allora. Nelle parole di Uri Schwartzmann, sergente e riservista nei carri:

Niente ti prepara a entrare in una città martellata dai bombardamenti e piena di civili. Gli aerei e la marina avevano bombardato la città prima che entrassimo. Quando sono arrivate le forze di terra, la città era in fiamme. Si vedevano le strade in fiamme come su un set di un film catastrofico, e le auto polverizzate; un odore di carne bruciata riempiva l'aria e si infiltrava ovunque; qua e là piccoli gruppi di civili vagavano senza meta, in stato di shock, in mezzo a questa devastazione incomprensibile ${ }^{19}$.

Questa descrizione richiama diverse scene di distruzione e desolazione che si vedono attraverso il mirino del carro armato di Lebanon al suo ingresso

\footnotetext{
17 Ivi, 41:40-43:26.

${ }^{18}$ I. Gal - I. Hammerman, De Beyrouth à Jénine cit., p. 74.

${ }^{19}$ Ivi, p. 41.
} 
nei primi centri abitati nel sud del paese; e vi sono altri elementi che rendono questo film una lunga testimonianza sulle esperienze dei soldati, quelli del carro armato protagonista del film o gli altri protagonisti delle operazioni militari vere e proprie. Uno di loro, Amir Tal, racconta della mancanza di visione di insieme («non hai idea di che cosa succeda (...). Tutto quello che vedi è attraverso una piccola lente, e non esiste di mettere la testa fuori»); un altro Ilan Hauser, capitano riservista, allarga questo discorso alla sfera politica, ricordando che non si aveva alcun modo di sapere se era «il ministro della difesa che decideva e diceva all'esercito che cosa fare, o se si trattava di decisioni del capo di stato maggiore o del comando generale nord». Si ritrova questa stessa situazione di ignoranza generale anche in Lebanon, per esempio nella domande del cannoniere, cui era stato detto che la guerra sarebbe durata poco tempo, mentre un superiore parla di un certo numero di missioni da svolgere entro le tre settimane successive. Il già citato Asher Fahan sembra dare voce all'immagine del carro armato di Lebanon che si perde e si ritrova nello stesso campo di girasoli in cui il film ha inizio e fine, quando ricorda che «in verità durante tutta questa guerra non abbiamo mai ricevuto il bilancio della situazione, non sapevano che cosa i nostri avessero già conquistato o dove si trovassero» ${ }^{20}$. La stessa mancanza di comunicazione tra ranghi e tra settori dell'esercito viene chiamata in causa anche da Ari Folman in Valzer con Bashir nella sua ricostruzione di come l'Idf abbia troppo lentamente messo insieme le informazioni che rendevano evidente che si stava svolgendo un massacro a Sabra e Shatila. Come vedremo più avanti, la mancanza di comunicazione tra i vari settori dell'esercito è un tema che ritorna anche nei film relativi al 2000, anno in cui venne evacuata la cosiddetta Zona di Sicurezza.

Al centro di questi due film sulla guerra del 1982 sta il tema della memoria e del ritorno del rimosso. Valzer con Bashir ha intrecciato una serie di eventi storici al processo di recupero della memoria del regista, mentre la scrittura della sceneggiatura di Lebanon ha rappresentato il primo passo di Samuel Maoz per ritrovare la memoria di quegli eventi, condizione necessaria per guarire e tornare a vivere. Come scrive il regista:

La guerra è scoppiata nel giugno 1982. Quando sono tornato, mia madre mi ha abbracciato, piangendo e ringraziando (...) Dio di avermi fatto tornare sano e salvo. All'epoca non aveva idea che non ero tornato a casa sano e salvo. In realtà non ero proprio tornato. Non aveva idea che suo figlio era morto in Libano e che stava in quel momento abbracciando un involucro vuoto ${ }^{21}$.

Nella cartella stampa Maoz ha descritto alcuni passaggi del suo percorso, dall'impossibilità di elaborare il trauma di quella sua partecipazione alla guerra per vent'anni - in cui era paralizzato da un punto di vista professionale, «sotto pressione da un punto di vista economico», sofferente di «attacchi

\footnotetext{
20 Ivi, rispett. pp. 85, 126, 29.

${ }^{21}$ www.sonyclassics.com/lebanon/lebanon_presskit.pdf, p. 6, accesso del 14 aprile 2012.
} 
di rabbia violenta», in cui si sentiva «passivo e privo di senso di responsabilità al punto da impazzire» - fino al progressivo allontanamento da «quel ragazzo che stava nella sua memoria» e lo «strano senso di euforia» con cui ha scritto la sceneggiatura in tre settimane, «quando sentiva di non aver più niente da perdere». A completare il processo di guarigione, l'infezione al piede all'inizio delle riprese causata dall'uscita di cinque schegge, «ultima testimonianza della guerra in Libano che il mio corpo aveva deciso di espellere» dopo 24 anni. I termini usati per descrivere il risultato di questo processo sono: uscita dall'ibernazione, nuovo sangue che scorre nelle vene, riabilitazione, conquista di se stessi ${ }^{22}$.

La realizzazione di Lebanon ha costituito il risultato di questo recupero della memoria e dell'elaborazione del trauma individuale, mentre le tappe di questo stesso processo di riappropriazione della memoria di eventi traumatici rappresenta il soggetto del film di Folman, a partire dal racconto di un incubo di un amico che apre Valzer con Bashir - 26 cani che abbaiano sotto la sua finestra, tanti quanti quelli che ha ucciso all'entrata dei villaggi perché non dessero l'allarme. A partire da questo sogno - che spinge il regista a ricercare la memoria rimossa del proprio ruolo in quella guerra - attraverso una ricerca condotta con l'aiuto di commilitoni, amici e terapeuti, a Folman torna l'immagine della propria presenza nelle immediate vicinanze di Sabra e Shatila nei giorni del massacro. Partendo da queste premesse, Raz Yosef ha scritto che questi due film rappresentano la fine della memoria collettiva della società israeliana, a favore della ricerca di una memoria individuale che era stata espunta dalla narrazione storica nazionale. È questo distacco dalla memoria collettiva nazionale che, sempre secondo Yosef, colloca «i film in un mondo caratterizzato da una confusione persistente tra il contesto storico e le impressioni private e soggettive, un mondo senza tempo, fatto di sogni, fantasie, allucinazioni e miti» 23 .

Sia Yosef che Raya Morag, un'altra critica israeliana, hanno preso le mosse da Dominick LaCapra e dalla sua distinzione tra perpetrator, bystander e victim, termini che ho qui tradotto come «esecutore», «spettatore/astante» e «vittima». Secondo questa lettura, non tutti coloro che sono stati traumatizzati da un evento ne sono vittime in egual misura: il trauma della vittima è di tipo psicologico e socio-politico, mentre il trauma dello spettatore e dell'esecutore è anche di tipo etico. Come scrive LaCapra: «bisogna che il trauma dell'esecutore venga riconosciuto in quanto tale e in qualche modo elaborato se gli esecutori devono prendere le distanze da una precedente implicazione in ideologie e pratiche mortali» 24 .

Non è del tutto immediato o agevole utilizzare questa terminologia nel caso dei soldati israeliani, proprio per le implicazioni dei termini «esecutore»e

22 Ivi, pp. 3-4.

${ }^{23}$ R. Yosef, The Politics of Loss and Trauma in Contemporary Israeli Cinema cit., p. 4; cfr. anche Id., War fantasies: memory, trauma and ethics in Ari Folman's Waltz with Bashir, «Modern Jewish Studies», 9 (2010), n. 3, pp. 311-26.

${ }^{24}$ D. LaCapra, Writing History, Writing Trauma, Johns Hopkins UP, Baltimore 2001, p. 79. 
«vittima» nel contesto della storia ebraica (e non solo), diasporica e non, del $\mathrm{XX}$ secolo. In questo senso ci viene in aiuto il lavoro di Raya Morag, che ha introdotto un'ulteriore distinzione - il persecuted perpetrator - per vari motivi, tra cui alcune necessità linguistiche proprie dell'ebraico. Morag suggerisce che «il trauma dell'esecutore esplode precisamente perché il complesso di persecuzione è stato interiorizzato» $25 \mathrm{e}$ che sia tanto più profondo in quanto risultato di un corto circuito tra le immagini interiorizzate della persecuzione nazista e il loro ritornare in vari teatri di guerra combattuti dell'Idf, specialmente in situazioni di combattimenti tra i civili.

In Valzer con Bashir questa distinzione funziona rispetto alla storia dei personaggi, a cominciare da Ari Folman, figlio di sopravvissuti ad Auschwitz. Come gli dice un amico psicoanalista che il protagonista va a trovare una mattina all'alba: «il tuo interesse per il massacro [di Sabra e Shatila] deriva da un altro massacro. Il tuo interesse per quei campi riguarda in realtà gli "altri" campi [di sterminio]» ${ }^{26}$. Allo stesso modo, la storia della sua prima licenza - menzionata sopra per illustrare le differenze tra la guerra del Libano e le precedenti - richiama le storie di altre licenze di soldati su altri fronti, sempre della seconda guerra mondiale, così come gli erano state trasmesse dal padre. Per cercare di confortarlo, questi gli aveva raccontato che «durante la sua guerra (...) i soldati russi di Stalingrado avevano ricevuto 48 ore di licenza dopo un anno al fronte». Partiti in treno, erano potuti solo scendere sul binario per abbracciare le mogli per poi risalirvi e tornare al fronte ${ }^{27}$. Una sovrapposizione di questo genere emerge ancora nelle parole di Ron Ben-Yishai, il corrispondente di guerra israeliano in Libano che per primo avvertì Ariel Sharon che vi era un massacro in corso a Sabra e Shatila, e il primo ad entrare nei campi dopo il massacro: «Conosci la foto del Ghetto di Varsavia? Quella del bambino con le mani alzate? (...) Questa era l'immagine, con donne, vecchi e bambini che camminavano» 28 . Si tratta in questo caso di una di quelle immagini che ben rappresentano quelli che Allen Meek chiama i traumi culturali iconici (iconic cultural traumas) - immagini di guerra, genocidio, tortura e via dicendo - che vengono rivissuti e ri-raccontati per mezzo di numerosi documentari e drammatizzazioni e che egli vede come fondamentali nel dare vita a forme contemporanee di relazione tra storia e memoria, e quindi di politica ${ }^{29}$.

${ }^{25}$ R. Morag, Current Israeli Documentary Cinema and the Trauma of the Israeli Soldiers, www.youtube.com/watch? $\mathrm{v}=\mathrm{hdkIm} 00 \mathrm{WkIg} \&$ feature=relmfu; online papers from the Trauma, Testimony, Discourses, Genre, Patterns and Innovation International Conference, February 21-24, 2011, Tel Aviv University, accesso del 22 maggio 2012. Qui Morag discute quale possa essere il termine più indicato per il perpetrator di LaCapra, visto che da un lato kovesh è associato alla persecuzione nazista, mentre le due alternative, me'avel o meholel (colui che fa del male o violatore) sono per contro prive di qualsiasi spessore storico.

26 A. Folman, Waltz with Bashir, 1.05:15-1.05:21.

27 Ivi, 49:33-50:19.

28 Ivi, 1:17:43-1:18:00.

${ }^{29}$ A. Meek (ed.), Trauma and Media: Theories, Histories and Images, Routledge, Florence (KY) 2009, pp. 6 e 8. La storia del bambino (Tzvi Nussbaum) che esce con le mani alzate dal 
Questo tipo di costruzione culturale è stata giudicata severamente da Yosef, che ha visto proprio in questo collegamento tra Sabra e Shatila e il trauma della Shoah un meccanismo autoassolutorio del film nei confronti della responsabilità del governo (e dell'esercito) israeliano in Libano: in questo modo, il personaggio Folman è costruito come una vittima innocente della storia (figlio di sopravvissuti) che contro la sua volontà diviene spettatore di eventi traumatici su cui non ha saputo/potuto intervenire. In questo senso la presenza israeliana a Beirut nel 1982 viene data per scontata, senza alcun intervento critico ${ }^{30}$. Come vedremo nella sezione successiva, grazie al lavoro di Nurit Kedar, la presenza dell'Idf a Beaufort non è stata considerata in maniera altrettanto naturale.

\section{La caduta dell'ultima fortezza tra mito e realtà: eroi ed anti-eroi}

Tredici soldati non è un romanzo autobiografico e, come l'autore Ron Leshem spiega nel post scriptum, è basato su lunghe interviste con i soldati che erano stati di stanza a Beaufort. Questi stessi uomini sono stati poi intervistati e filmati in Wasted; a questo si aggiunga che le interviste per quest'ultimo film hanno avuto luogo sullo stesso set usato in Beaufort. Tredici soldati, Beaufort e Wasted sono quindi tre opere strettamente collegate a partire dalle testimonianze dirette di chi prese parte a quell'esperienza: insieme, rivelano tre aspetti della stessa storia e tre modi di leggere lo stesso mito nazionale. Con i suoi durissimi riferimenti alla realtà militare di quell'esperienza per voce dei suoi protagonisti diretti, Wasted rivela inoltre che il grado di finzione che si presume nel romanzo è in realtà molto basso.

Il castello crociato di Beaufort, collocato in territorio libanese qualche chilometro oltre il confine nord (est) di Israele, fu conquistato dall'Idf durante il primo giorno di guerra, in circostanze militari ancora oggi da chiarire. Da allora, la storia di Beaufort ha acquisito i tratti della leggenda; come dice Liraz Liberti, il comandante del forte, in Tredici soldati: «E Goni, che ha conquistato il Beaufort? È un eroe, perfino i bambini lo conoscono» ${ }^{31}$. In mancanza di studi storici che contribuiscano alla decostruzione di questa vicenda, è interessante per adesso vedere come essa sia stata rappresentata nella letteratura e nel cinema israeliani a qualche anno dalla sua conclusione ${ }^{32}$.

ghetto di Varsavia è stata scritta da D. Porat, The Boy: A Holocaust Story, Melbourne UP, Carlton (Vic.) 2010; sulla rappresentazione della Shoah attraverso immagini di bambini con particolare riferimento alla cultura statunitense cfr. M.M. Anderson, The Child Victim as Witness to the Holocaust: An American Story?, «Jewish Social Studies», 14 (2007), n. 1, pp. 1-22.

${ }^{30} \mathrm{R}$. Yosef, The Politics of Loss and Trauma cit., p. 140.

${ }^{31}$ R. Leshem, Tredici soldati cit., p. 263. Il riferimento è al maggiore Guni Harnick.

${ }^{32}$ Cfr. anche la rivisitazione del celebre Chateau des Pyrénées di René Magritte da parte dell'artista Aaron Barnea nell'opera Beaufort - following Magritte. La rocca che è sospesa sul mare in Magritte è qui sospesa sul deserto; il castello del primo è sostituito con Beaufort nel secondo; le nuvole sull'orizzonte di Magritte sono assenti in Barnea, che avvolge il suo 
Come accennato sopra, l'operazione militare con cui l'Idf ha conquistato Beaufort ha avuto contorni ambigui: secondo i giornalisti Ze'ev Schiff e Ehud Ya'ari, autori di un volume fortemente polemico sulla guerra del Libano, scritto mentre la guerra era in corso, Beaufort non rappresentava una postazione di particolare rilevanza militare, nonostante la sua posizione dominante (altitudine: $717 \mathrm{~m}$ ) da un punto di vista geografico e strategico. Da Beaufort non si era sparato contro le cittadine israeliane oltre confine (circa 5 $\mathrm{km}$ ) prima o durante le prime ore di guerra; l'intera zona sottostante invece popolata di villaggi cristiani e controllata dal maggiore Saad Haddad (leader delle forze cristiane addestrate da Israele) - aveva sofferto del controllo esercitato dai guerriglieri della forza Kastel dell'OLP arroccati nel forte. L'operazione che portò alla conquista di Beaufort non era stata prevista nei piani dell'Idf relativi alle prime 24 ore di guerra, né la situazione militare sembrava richiederla, visto che, con l'avanzare dell'Idf verso nord, il forte sarebbe rimasto isolato, e sarebbe quindi caduto in maniera più agevole in un secondo momento, se non addirittura abbandonato ${ }^{33}$. L'ordine di non attaccare Beaufort passato dal capo di Stato maggiore Rafael Eitan al generale del Comando Nord Amir Drori - sembra tuttavia non aver mai raggiunto il comandante della Brigata Golani che guidò l'attacco o, secondo un'altra versione, sembra essere arrivato con due ore e mezza di ritardo, quando ormai il forte era caduto. Nella battaglia vennero uccisi tra i 10 e i 24 palestinesi e 6 soldati dell'Idf, le cui famiglie chiesero l'apertura di un'inchiesta dopo qualche mese ${ }^{34}$.

Come questi particolari mettono in evidenza, la storia recente di Beaufort non è quindi esente da controversie militari, politiche e storiografiche, ed è partendo dalla valenza simbolica attribuita a questo luogo - che come recita la didascalia iniziale di Wasted «è entrato nel mito per generazioni di israeliani» - che si riesce a comprendere alcune delle modalità con cui è stato recentemente rappresentato. La conquista di Beaufort era una delle ossessioni di Begin - un primo ministro «nato tra la mania e la depressione» e «con un debole collegamento con la realtà» 35 - perché simboleggiava il controllo che $\mathrm{i}$

Beaufort con delle pennellate e chiazze rosse che discendono sul deserto sottostante. Cfr. www.arteria.co.il/artwork_show_743.html, accesso del 2 novembre 2012. Il pittore Aaron Barnea è il padre di Noam Barnea, sergente maggiore e sminatore, caduto il 12 aprile 1999 (cinque giorni prima del congedo) a Beaufort nel tentativo di aprire la strada che portava al forte. Su di lui è costruito il personaggio di Ziv che apre sia il romanzo Tredici soldati che il film Beaufort. Aaraon Barnea è tra i fondatori del Parents Circle-Family Forum, ong mista israelopalestinese che riunisce i parenti di primo grado delle vittime del conflitto. Cfr. anche il filmato in tre parti intitolato Noam Barnea, in www.youtube.com/watch? $=\mathrm{aBJ} \mathrm{xB} 5 \mathrm{iONvQ} \&$ feature =relmfu, accesso del 2 novembre 2012 .

33 Z. Schiff-E. Ya'ari, Israel's Lebanon War, Simon \& Schuster, New York 1984, pp. 125-31.

34 Solo nel 2006 il Dipartimento di storia dell'Idf ha compilato un rapporto di un centinaio di pagine sulle dinamiche militari dell'attacco al forte, senza tuttavia affrontare il problema di dove si fosse interrotta la catena di comando. Il rapporto, a tutt'oggi riservato, fu mandato alle famiglie dei caduti e porta la firma del generale di Brigata Dr. Dani Asher.

35 Y. Sarid, 1,216 dead - who bears the guilt?, «Haaretz», 8 giugno 2012. 
palestinesi esercitavano sulla zona, e Arafat stesso aveva visitato il castello qualche settimana prima dell'inizio della guerra. Vista la spettacolarità della sua posizione inoltre, Begin vedeva Beaufort come un obiettivo di prestigio con cui silenziare l'opposizione interna; si ricorderà che l'obiettivo dichiarato della guerra era il respingimento della guerriglia palestinese oltre il fiume Litani $(40 \mathrm{~km})$ - confine che peraltro era stato superato già dopo il primo giorno di combattimenti - e Beaufort si trova non a caso subito al di là di questo fiume ${ }^{36}$.

Ancora prima che la conclusione della vicenda di Beaufort nel 2000 - e la sua rappresentazione letteraria e cinematografica - aggiungessero un ulteriore strato narrativo in chiave eroica a questa vicenda, i fattori che avevano favorito la creazione del mito erano già presenti: il sito denso di storia e la sua spettacolarità, la rapidità dell'operazione, un nemico storico che lo occupava, i caduti israeliani e la lettera aperta che il padre di uno di loro - Yaacov Gutterman - scrisse a Begin, Sharon ed Eitan, accusandoli di aver assassinato il figlio e gli altri soldati.

Yaacov Gutterman non era stato soltanto il padre di Raz Gutterman; era anche il figlio di un combattente del ghetto di Varsavia, un sopravvissuto alla Shoah, che descriveva Raz come un eroe nella migliore tradizione classica, «forte, bellissimo, retto e onesto», ma soprattutto come «un anello nella catena della storia assieme ad altri, la realizzazione del rinnovamento del nostro popolo». La lettera di Gutterman era molto dura:

Anche i bambini sanno che avete cercato una ragione per entrare in Libano e per istigare la prima guerra che non era difensiva. Avete cercato di rimediare a tutti i vostri fallimenti, inadeguatezze e frustrazioni, a tutte le vostre mancanze politiche con questa dubbia vittoria militare. Il mio amato figlio Raz e i suoi amici sono stati mandati con la loro unità con grande fretta e frenetica irresponsabilità alla sanguinosa battaglia per prendere il forte di Beaufort. (...) Quanti anni di questa generazione ci sarebbero voluti ai terroristi palestinesi per uccidere e ferire tanti soldati israeliani quanti ne avete uccisi voi in una settimana di questa guerra maledetta? ${ }^{37}$

Per quanto con la sua lettera Gutterman rifiutasse in toto le ragioni della guerra utilizzate da Begin, Sharon ed Eitan, il suo tragico percorso familiare lo inseriva nella narrazione più tradizionale del sionismo che, come gli studi di Yael Zerubavel, Idit Zertal e altri hanno dimostrato, vedeva la fondazione dello Stato di Israele come il riscatto da una storia di persecuzione diasporica di cui la Shoah era stato il punto più basso e la rivolta del ghetto di Varsavia il momento più eroico ${ }^{38}$. Come noto, Begin impostò anche la guerra del 1982

${ }^{36}$ Sulla storia della controversa conquista di Beaufort, cfr. S. Fogelman, Casting a Giant Shadow, «Haaretz Week's End», 1 giugno 2012, pp. B1-B2.

37 Y. Gutterman, An open letter to Menachem Begin, Ariel Sharon and Raphael Eitan and the ministers who voted for the war in Lebanon (22 giugno 1982), «New Outlook», agosto-settembre 1982, pp. 58 e 62. Intervista dell'A. a Y. Gutterman, Kibbutz Ha-Ogen, 22 gennaio 2007. In Tredici Soldati Raz Gutterman è Razi Gutman.

38 Y. Zerubavel, The "Mythological Sabra" and Jewish Past: Trauma, Memory, and Contested Identities, «Israel Studies», 7 (2002), n. 2, pp. 115-44; I. Zertal, Israele e la Shoah. La nazione e il culto della tragedia, Einaudi, Torino 2007. 
attingendo a questo stesso repertorio; per esempio scrivendo al presidente degli Stati Uniti Ronald Reagan di aver mandato l'Idf in Libano per catturare (il nuovo) Hitler (Yasser Arafat) nel suo bunker a Beirut, che provocò la famosa risposta pubblica dello scrittore Amos Oz: «Signor primo ministro, Adolf Hitler è già morto» ${ }^{39}$.

A differenza di Valzer con Bashir e in parte di Lebanon, che si concentrano sul 1982 e che, come abbiamo visto, risentono ancora di questa sovrapposizione concettuale, né Tredici Soldati, né Beaufort né Wasted - che si riferiscono a un periodo successivo - fanno ricorso a questo tipo di repertorio. Non si trovano qui figli di sopravvissuti, eroi del ghetto di Varsavia o richiami alla persecuzione antiebraica in Europa. Abbiamo invece un gruppo abbastanza eterogeneo di soldati che vengono rappresentati sempre più intrappolati in una postazione isolata in territorio nemico, a difendere un avamposto di un esercito definito nel romanzo «immerso nella tattica senza (...) la strategia» ${ }^{40}$. Alcuni dei personaggi di Tredici soldati, e in particolare il comandante Liraz, si mantengono all'interno della narrazione eroica; in più momenti Liraz si sofferma con malinconia sul pensiero che il suo nome sarà associato a quello dell" "ultimo comandante" del forte.

Per quanto Beaufort si proponga come trasposizione di Tredici soldati, le due rappresentazioni di questa stessa vicenda sono diverse: il primo sottolinea l'aspetto eroico della situazione, e mette il luce l'idea del manipolo di uomini asserragliati in una fortezza in territorio nemico costantemente sotto attacco; in questo senso, il film di Cedar lascia ampio spazio a una rappresentazione nazionalpopolare che ripropone in chiave contemporanea miti nazionali ormai consumati, che tuttavia continuano a avere una certa risonanza nel pubblico. Secondo questa lettura, mascherata dietro un film d'azione/di guerra, la fortezza di Beaufort altro non è che la nuova Masada, che come noto rappresenta uno dei miti fondativi nazionali israeliani e che descrive il senso di assedio con cui lo stato e la società israeliana hanno storicamente rappresentato se stessi ${ }^{41}$. Il romanzo Tredici soldati invece - che ugualmente

39 A. Oz, Hitler kvar met adoni rosh ha-memshalah, in J. Kafri, Chatziat Gvul: Shirim Milhemet Levanon, Sifriat Poalim, Tel Aviv, 1983, p. 71 (ebraico; «Hitler è già morto, signor primo ministro», in Attraversare il confine: Poesie della Guerra del Libano); cfr. anche Y. Bauer, Fruits of Fear, «Jerusalem Post», 3 giugno 1982, p. 8 e Z. Mankovitz, Beirut is not Berlin, ivi, 4 agosto 1982, p. 8.

${ }^{40}$ R. Leshem, Tredici soldati cit., p. 321

${ }^{41}$ Il De Bello Judaico di Giuseppe Flavio racconta, tra le altre cose, la storia dell'assedio romano a Masada, la fortezza costruita e fortificata da Erode tra il 37 e il 31 a.C. Qui dal 66 d.C. era asserragliato un gruppo di estremisti (i Sicarii, gruppo dissidente dei già estremisti Zeloti) che resistette all' assedio, preferendo darsi la morte che consegnarsi ai romani che riuscirono a espugnarla nel 73 d.C, tre anni quindi dopo la caduta del Tempio di Gerusalemme che, convenzionalmente, segna l'inizio della diaspora ebraica. Sulla storia di Masada come mito fondante nazionale in Israele cfr. B. Schwartz - Y. Zerubavel - B.M. Barnett - G. Steiner, The recovery of Masada. A study in Collective Memory, «The Sociological Quarterly», 27 (1986), n. 2, pp. 147-64 e il classico N. Ben-Yehuda, The Masada Myth: Collective Memory and Mythology in Israel, University of Wisconsin Press, Madison 1995. 
descrive le difficoltà militari di questo gruppo - è più articolato, e riesce a proporre una parziale decostruzione di questo stesso mito.

I soldati del romanzo sono un gruppo eterogeneo; per un Liraz che risponde ai canoni della rappresentazione militare di un giovane comandante, ve ne sono altri che se ne differenziano profondamente, per esempio nelle opinioni politiche, o nel percorso di vita civile che hanno scelto. È il caso di Ziv che, prima di morire nel tentativo di sminare l'accesso all'unica strada del forte, vestiva una maglietta con la scritta «usciamo dal Libano in pace», uno degli slogan del movimento delle Quattro Madri, ong fondata nel 1997 per il ritiro israeliano dal Libano, e che Liraz considerava «una banda di femmine isteriche che si scatenavano agli incroci (...) che cercavano di convincere chiunque fosse disposto ad ascoltarle che noi, i combattenti, non eravamo altro che carne da cannone in una guerra inutile» ${ }^{42}$. È il caso di Spitzer, che studia teatro nella vita civile, e che durante un turno di guardia viene sentito recitare uno dei monologhi più famosi dell'Enrico $\mathrm{V}$ di Shakespeare, in una sovrapposizione di situazioni che sottolinea ulteriormente l'idea del manipolo di eletti che si appresta a combattere la battaglia campale ${ }^{43}$. È il caso anche di River che, pur fulminato con lo sguardo da Liraz, non esita a sfidare il generale di brigata Kaplan in visita alla postazione, affermando davanti a tutti di non essere riuscito davvero a convincersi che valesse la pena perdere tutto ciò che questo gruppo aveva perso per difendere un forte che sarebbe stato comunque evacuato di lì a poco. $\mathrm{O}$ del sergente Oshri, punto di riferimento per il gruppo, che prima di andarsene a conclusione del suo servizio militare, rivela di essere stato destinato a Beaufort dall'impiegato al servizio di leva e non per scelta, né perché suo «padre era un comandante che ti ha tirato su per seguire la sua strada nello spirito pionieristico e sionistico» ${ }^{44}$. A questi personaggi si può ancora aggiungere il già menzionato Kaplan. Il generale di brigata da un lato appare prigioniero di Beaufort e del suo mito: la sua vita adulta e il suo intero servizio militare erano stati connessi con Beaufort, i suoi «più cari amici erano rimasti» in questo luogo, e aveva «continuato sulla loro strada» per ricordarli sempre. Dall'altro tuttavia, si trovava a Beaufort la prima sera della Pasqua ebraica non tanto per trasmettere ai più giovani la sua storia, ma per confrontarsi con una generazione di soldati diversa dalla sua «che fa domande. Ci costringete a rispondervi. È un bene». Come dice egli stesso:

42 R. Leshem, Tredici soldati cit., 133. Il movimento fu fondato da Rachel Ben-Dor, Miri Sela, Ronit Nachmias, and Zahara Antavi; cfr. www.4mothers.org.il/mothers.htm, accesso del 1 novembre 2012.

${ }^{43}$ W. Shakespeare, Enrico V, atto IV, scena III: «Noi pochi. Noi felici, pochi. Noi manipolo di fratelli: poiché chi oggi verserà il suo sangue con me sarà mio fratello, e per quanto umile la sua condizione, sarà da questo giorno elevata, e tanti gentiluomini ora a letto in patria si sentiranno maledetti per non essersi trovati oggi qui, e menomati nella loro virilità sentendo parlare chi ha combattuto con noi questo giorno di San Crispino!»

${ }^{44}$ R. Leshem, Tredici soldati cit., pp. 195-96. 
È tutto cominciato qui. Non solo gli eroi e i simboli, ma anche la spaccatura fra gli israeliani, le dimostrazioni di protesta, il movimento pacifista e Peace Now. Qui, per la prima volta, sono emersi i pensieri sulla futilità di tutto questo. Ma credo che non siano mai stati così radicati fra i soldati come adesso ${ }^{45}$.

Per quanto Kaplan sia un personaggio solo letterario, ben rappresenta la visione politica della vecchia élite militare contrapposta a quella di Begin e Sharon, rappresentanti di una classe dirigente assetata di potere, intrappolata nel mito di se stessa, del militarismo e del richiamo mortale di Beaufort, dal 1982 per i successivi diciotto anni. Nell'annunciare che la decisione di evacuare il forte è stata ormai presa, Kaplan riconosce che il dubbio - rifiutato qualche pagina prima dal più inesperto comandante Liraz $^{46}$ - e la protesta stanno crescendo tra i soldati (e non solo).

Del dubbio e della protesta si trova traccia in vari momenti del racconto: nella storia di un articolo di giornale che compare appeso in mensa, si cui qualcuno dei soldati aveva scritto a pennarello «Sei un mito!»; l'articolo riportava un'intervista a un paracadutista che aveva dichiarato di non voler morire senza una buona ragione. Nel racconto dell'impatto che vari servizi televisivi avevano avuto sui soldati di Beaufort, al funerale di uno di loro il padre aveva gridato: «il governo ha abbandonato i nostri migliori soldati. (...) Questa non è una vera guerra, è un'assurdità. Fino a quando?». In un altro servizio televisivo alcuni degli stessi protagonisti erano stati intervistati ed un sergente rivolgeva un appello al primo ministro Barak perché evacuasse il Libano «adesso», senza aspettare la data prevista; davanti al microfono Spitzer aveva dichiarato: «se qualcuno ti dice che non ha paura, è un bugiardo»; il telegiornale faceva quindi sentire le voci reali dei soldati, che si contrapponevano alle dichiarazioni ufficiali, che non potevano che riportare il messaggio del capo di stato maggiore «Stiamo sconfiggendo Hezbollah»:

Riportateci a casa. (...) Non vogliamo essere l'ultima vittima in Libano, nessuno di noi vuole morire senza un motivo. (...) Voi avete influenza. Per favore aiutateci a uscire di qui. Chi sta in Libano sa bene che non abbiamo alcuna possibilità di vincere (...) Da inseguitori ci siamo trasformati in prede (...)non siamo altro che bersagli, non rispondiamo, stiamo solo ad aspettare che ci colpiscano. In dieci giorni abbiamo perso sei compagni. Cosa ci stiamo a fare qui?

Se in Tredici soldati il dubbio si trasforma lentamente nella certezza che il ritiro sia un fattore positivo, Beaufort è meno esplicito. Non a caso Kaplan ritorna anche sulla storia dell'ordine di conquistare Beaufort di cui aveva già parlato Spitzer, attirandosi l'accusa di aver «profanato la memoria dei morti», quelli del 1982 e i successivi.

\footnotetext{
45 Ivi, pp. 320 e 322.

46 «I soldati non si devono fare domande del genere; non se ne parla. Con idee simili in testa, sarai un combattente di merda; saranno i dubbi ad ammazzarti, te e i tuoi amici» (ivi, p. 215).
} 
Ma tu lo sai che non dovevano conquistarlo questo monte, che non è stato intenzionale? (...) Ancora oggi nessuno capisce come sia successo. Quando è scoppiata la guerra (...) il Comando ha dato ordine di annullare l'operazione, di non attaccare, ma l'ordine si è perso per strada, da qualche parte, fra gli ufficiali al Comando e quelli della divisione; qualcuno si è dimenticato di trasmetterlo. (...) È stata una battaglia inutile.

Futilità, battaglie senza senso, senso di abbandono, stanchezza, crollo del mito, sono questi i termini che ricorrono nelle testimonianze di chi era di stanza a Beaufort rielaborate in Tredici soldati e che è possibile ascoltare direttamente dai protagonisti in Wasted. È quindi inevitabile che il romanzo e il documentario presentino delle corrispondenze: in Tredici soldati, il personaggio River non aveva dubbi sul fatto che non vi fosse alcuna necessità di stazionare a Beaufort:

Non ha niente a che vedere con il nord di Israele. Noi siamo qui soltanto per pararci il culo. Tutto qua. Ce ne stiamo bloccati a Beaufort, isolati da tutto, a beccarci razzi, colpi di mortaio ordigni esplosivi, rischiamo la vita, e solo per poter continuare a restare sul Beaufort. La missione è tutta qui. (...) Non volevamo far altro che aprire la fottuta strada [l'operazione in cui è morto il personaggio Ziv, alias Noam Barnea]. La fottuta via d'accesso che ci riporta in Israele. Dimmelo tu che sei ufficiale, che cazzo ci facciamo qui? ${ }^{47}$

A queste parole fa eco Koris, l'infermiere del gruppo, intervistato per Wasted:

La cosa che faceva più paura è che non c'era un nemico che potevamo vedere. Non vedevamo gli Hezbollah. Chi erano? L'unica cosa che conoscevamo erano le esplosioni, i colpi di mortaio e i missili che ci colpivano incessantemente. Ma chi erano? Non li abbiamo mai visti in faccia. Il nemico ha vinto. Sono riusciti a uccidere Noam [Barnea] e sono quasi riusciti a colpire il resto di noi in quell'imboscata. Ma non sapevamo chi erano. Quando uscivamo per montare la guardia, a chi e a che cosa stavamo facendo la guardia? Fuori era tutto buio. Abbiamo cominciato a sentire che stavamo facendo la guardia a noi stessi. (...). Perché eravamo lì? Per proteggere le città del Nord che erano tre, quattro, cinque $\mathrm{km}$ dietro di noi? In pratica stavamo cercando di proteggere la nostra postazione e noi stessi così da uscirne vivi fino a che la missione non fosse terminata 48

Al soldato che nel romanzo diceva «non siamo altro che bersagli, stiamo solo ad aspettare che ci colpiscano» fanno ugualmente eco alcuni dei protagonisti di Wasted: «Sono stato in Libano tre volte e non ho mai visto un terrorista; mai in vita mia un terrorista Hezbollah». E ancora:

Non so se abbiamo combattuto; non mi ricordo di aver combattuto. Mi ricordo che facevo quello che mi ordinavano ma non ho mai visto nessuno contro cui combattere, nessuno a cui sparare. Io ero quello a cui sparavano addosso ${ }^{49}$.

\footnotetext{
47 Ivi, rispett. pp. 249-51, 289-91, 263-64 e 161.

48 N. Kedar, Wasted, 2007, 56:07-56:30.

49 Ivi, 19:28-19:49; 29:52-30:24.
} 
Le testimonianze che si possono ascoltare in Wasted riguardano tutta una serie di aspetti operativi e di azioni militari che non risparmiano i particolari della devastazione fisica e psicologica di una guerra, tra cui arti che vengono recisi, teste che rotolano, il terrore di rimanere invalidi, il sangue che scorre copioso e la morte di alcuni. Questi aspetti sono presenti anche in Tredici soldati e, in misura minore, in Beaufort, ma sono stati mitigati in entrambi per esigenze di finzione artistica. Ciò che tuttavia divide il documentario dal romanzo e dal film è il carattere più diretto di testimonianza di Wasted rispetto a Tredici soldati o Beaufort. Da Wasted emergono infatti non solo le parole e i racconti, ma soprattutto le pause e i silenzi, che rivelano un gruppo di antieroi traumatizzati, vittime di un mito nazionale che si è richiuso su se stesso, e su di loro, e che ne ha schiacciati una buona parte ${ }^{50}$.

\section{Conclusioni}

Il set cinematografico è una delle immagini che più frequentemente ritorna nelle interviste, nelle dichiarazioni e nelle memorie dei soldati che hanno testimoniato della propria esperienza in Libano, nel 1982, nel 2000 e ancora nel 2006, dopo la Seconda guerra del Libano ${ }^{51}$. L'espressione «pareva di stare su un set di Hollywood» per descrivere la situazione - fumo, esplosioni, spari, caos, elicotteri, trasmissioni radio, i feriti e i morti - ritorna in tutti i testi qui presi in esame, così come in vari altri film o raccolte di testimonianze relative ad altri fronti di occupazione o di guerra, dai Territori Palestinesi durante la Prima o la Seconda Intifada all'operazione Piombo Fuso $(2008)^{52}$. Si tratta di un'immagine che offre diverse possibilità di interpretazione: da un lato è facilmente comprensibile anche dallo spettatore/lettore meno edotto di situazioni militari; dall'altro indica un tentativo di allontanare la realtà della guerra da sé. Lo stesso meccanismo si ritrova anche in Valzer con Bashir e Lebanon, per esempio nel modo con cui i due registi hanno scelto di tenere a distanza la guerra, nel primo per mezzo dell'animazione, e nel secondo attraverso il mirino del cannone. La guerra invece è ben presente in Wasted, che attraverso le parole - e i silenzi - dei testimoni non nasconde nessuno degli orrori; al contrario, richiama l'attenzione su uno degli elementi che ogni narrazione nazionale tende a celare, se non a censurare: lo

\footnotetext{
50 Per un'analisi di testimonianze traumatiche in alcuni recenti film israeliani tra cui Wasted, cfr. M. Simoni, From individual experience to the collective archive, from personal trauma to public memory. Accounts of War and Occupation in Israel, in J. Kohne et al. (eds), The Horrors of Trauma in Film: Violence, Void, Visualization, Cambridge Scholars Publishing, Cambridge, in corso di stampa.

51 A. Isaacs, Lebanon II.

52 Per testimonianze da questi due fronti cfr. rispettivamente i film Testimony di Shlomi Elkabetz (2011) e Concrete di Nurit Kedar (2011) analizzati in M. Simoni, 'From individual experience to the collective archive cit. Cfr. anche Testimonies di Ido Sela (1993) e il documentario To see if I am smiling di Tamar Yarom (2007), che presenta le interviste di alcune soldatesse durante la Seconda intifada (2000-2004).
} 
smembramento e la mutilazione del corpo del soldato. Non a caso, questo terrore torna insistentemente nelle parole dei soldati in tutti i film analizzati qui.

Valzer con Bashir, Lebanon, Beaufort, Wasted e Tredici soldati condividono ben più del teatro di guerra; vi si ritrovano atmosfere molto simili e molto spesso le stesse parole riecheggiano tra un testo e l'altro. In nessuno di questi testi, con la parziale eccezione di Beaufort, ci imbattiamo nella rappresentazione eroica di un protagonista o nella valorizzazione dell'eroe di guerra; come abbiamo visto, questo tipo di rappresentazione - che ha una serie di precedenti letterari importanti in Israele - non compariva neanche nella generazione precedente di film israeliani sul Libano. Emergono invece, in modo diverso a seconda delle atmosfere di ciascun film, tutta una serie di sentimenti che male si adattano a personaggi diretti al fronte o che si ritrovano in guerra: l'incertezza, il terrore, gli attacchi di panico, lo scarso controllo su proprio corpo. Si tratta di sentimenti e situazioni causate da diversi fattori singoli o da una loro combinazione: la giovane età, l'impreparazione psicologica delle reclute e la mancanza di motivazione della maggior parte di loro, l'invisibilità del nemico e la mancanza di una distinzione chiara tra militari e civili, la consapevolezza di non poter contare sul sostegno politico del fronte interno, l'apparente lontananza geografica e temporale dalla vita civile e dai suoi punti di riferimento, personali, familiari o professionali. Questi sentimenti - che compaiono in tutti e quattro i lungometraggi - si inseriscono con difficoltà nella struggente bellezza del paesaggio libanese, siano le piantagioni di banane di Valzer con Bashir, il campo di girasoli con cui si apre e si chiude Lebanon, o i panorami apparentemente infiniti di Beaufort. La paura, che non giocava un ruolo di particolare rilievo nei primi quattro film israeliani sul Libano prodotti tra gli anni ' 80 e '90, è diventata una delle protagoniste della rappresentazione di quella guerra trent'anni dopo. Accanto ad essa, recitano altre muse: la ricerca del rimosso e della memoria, l'elaborazione del trauma personale e il superamento del lutto, che dalla rappresentazione passano alla realtà con le interviste di Wasted.

Fondando la sua analisi su Valzer con Bashir e Lebanon, Raz Yosef ha sostenuto come proprio la prevalenza di questi ultimi elementi nella narrazione indichi che la società israeliana ha ormai imboccato la strada della memoria individuale, distaccandosi in maniera definitiva da quella visione (e memoria) collettiva che la aveva caratterizzata per lungo tempo. Questa individualizzazione della memoria porterebbe anche all'impossibilità di un dibattito nella sfera pubblica e politica, che coinvolga la società, la cultura e le istituzioni. Dopotutto, Valzer con Bashir e Lebanon sono due film strettamente autobiografici, nonché auto-terapeutici. Allargando tuttavia il campo di indagine fino a includere Tredici soldati, Beaufort e Wasted, la prospettiva cambia, anche se neanche il crollo della fortezza (e del mito) di Beaufort ha portato a una qualche forma di dibattito pubblico sulle responsabilità politiche della presenza israeliana in Libano dal 1982 al 2000. Presi assieme tuttavia, questi testi rivelano l'esistenza di un filone culturale-politico che si alimenta della raccolta ed elaborazione di testimonianze di soldati, e che coinvolge la letteratura, il cinema, organizzazioni non governative come Breaking the silence, B'Tselem $\mathrm{e}$ 
in parte Zochrot, alcuni settori del mondo accademico, una certa parte della stampa e via dicendo ${ }^{53}$. Si tratta di racconti e di storie che dialogano tra loro, e che si richiamano l'una con l'altra. Se, anche grazie al lavoro di queste ong, di registi indipendenti e di altri autori, allarghiamo ancora l'obiettivo oltre l'esperienza libanese, possiamo contare su numero crescente di testi, alcuni dei quali trasformati in lungometraggi, che da un lato denunciano le azioni dell'Idf sui vari fronti di guerra; dall'altro riportano le testimonianze anti-eroiche dei soldati che vi hanno combattuto. Oltre ai già citati Testimonies, Testimony e To see if I am smiling, ne è un esempio anche Z32 di Avi Mograbi (2007).

Le opere di argomento libanese - così come anche questi film e/o documentari ultimi citati - hanno una struttura narrativa che riprende una delle idee centrali del lavoro di Cathy Caruth e di altri, e cioè che la testimonianza traumatica - che sia ascoltata direttamente o rielaborata attraverso la finzione letteraria, cinematografica o attraverso tecniche di animazione - sia una fonte preziosa per decostruire alcuni pilastri della narrazione storica ufficiale ${ }^{54}$. Nel caso delle opere prese in esame, sono diversi i miti nazionali che scrittori e registi hanno continuato a demolire, inserendosi nella scia che altri scrittori e registi avevano cominciato a tracciare (Yehoshua, Oz, Kenaz, Gitai, Kovashvili ecc.). Nella maggior parte dei casi si tratta di un'opera di decostruzione che riguarda l'Idf, una delle istituzioni fondanti della società israeliana e dello Stato. I soldati di questi film sono quasi tutti anti-eroi: dal pubblicitario dei film degli anni ' 80 ai soldati asserragliati dentro Beaufort, tra cui alcuni che si fanno intervistare dai media per chiedere che la data dell'evacuazione venga anticipata; hanno quasi tutti paura, tranne quelli che sono morti. La rappresentazione dell'esercito è quella di un esercito che combatte tra i civili come nel 1982, o che combatte un nemico che non vede, come nel 2000, che si perde, che lascia indietro i propri uomini, in cui gli ordini si perdono nella catena di comando, che non spara un colpo da Beaufort. Da queste opere non ne esce meglio la leadership politico-militare; il ben noto filmato dell'arrivo di Begin e Sharon a Beaufort poche ore dopo la conclusione della battaglia (montato come introduzione a Wasted) mostra un primo ministro che, sceso dall'elicottero, commenta la qualità dell'aria di montagna e domanda all'esterrefatto soldato che lo accoglie se il nemico fosse dotato di mitragliatrici. Sono immagini che documentano l'impreparazione della classe politica e militare israeliana del 1982, e il suo distacco dalla realtà. Non si tratta solo di un messaggio di denuncia; a trent'anni di distanza, la letteratura e il cinema sembrano aver indicato una strada su cui la storiografia sta, con ritardo, cominciando a incamminarsi.

\footnotetext{
${ }^{53}$ Per i siti delle ong cfr. www.breakingthesilence.org.il, http://www.btselem.org, http://zochrot. org/en, accesso del 12 novembre 2012.

54 C. Caruth (ed.), Trauma. Explorations in Memory, The Johns Hopkins UP, BaltmoreLondon 1995, p. 4.
} 


\title{
Alla Moneda con Salvador Allende
}

\author{
Gennaro Carotenuto
}

At Moneda with Salvador Allende. The oral sources collected among the persons who were inside La Moneda Palace during the coup against Salvador Allende's government, on $11^{\text {th }}$ September 1973 , facilitate the study of historiographical problems such as the resistance from inside the palace, with the participation of the president himself, and the question of the armed defence of the popular government, in the circumstances of Allende's death. The thesis of the homicide is dismissed, and two different versions of the suicide proposed, that open the way to a series of considerations about how the Concertación reconstructed the figure of Allende.

Key words: Chile, Salvador Allende, Latin America, oral history, socialism Parole chiave: Cile, Salvador Allende, America latina, storia orale, socialismo

Oggetto di questo articolo è l'analisi e l'uso di fonti orali per lo studio della «battaglia della Moneda», l'11 settembre 1973 a Santiago del Cile'. Tale ricerca è effettuata usando la metodologia del racconto di vita ${ }^{2}$, in particolare di 9 testimonianze ${ }^{3}$ raccolte nella regione metropolitana e nella $\mathrm{V}$ regione (Valparaíso) che ruotano intorno alla figura di Salvador Allende e all'ultimo giorno della sua vita. Cinque degli intervistati accompagnarono il presidente nel Palazzo. Tre di essi appartenevano alla scorta del presidente, i Gap, «gruppo amici del presidente», l'apparato del Partito socialista che aveva in

1 Ó. Soto, El último día de Salvador Allende, Aguilar, Santiago 1998; I. González Camus, El día que murió Allende, Cesoc, Santiago 1988.

2 R. Fraser, La Historia Oral como historia desde abajo, «Ayer», 12 (1993), pp. 79-93; Id., La formación de un entrevistador, «Historia y Fuente Oral», 3 (1990), pp. 129-50; A. Portelli, L'uso dell'intervista nella storia orale, in E. Cento-L. Di Ruscio (a cura di), Didattica della storia dell'800 e del '900, Archilab, San Miniato 2005, pp. 58-67; S. Portelli, Storie orali: racconto, immaginazione, dialogo, Donzelli, Roma 2007; Id., Un lavoro di relazioni: osservazioni sulla storia orale, in www.aisoitalia.it/2009/01/un-lavoro-di-relazione; L. Passerini, Storia e soggettività. Le fonti orali, la memoria, La Nuova Italia, Firenze 1985.

${ }^{3}$ Alla prima citazione dell'intervistato se ne inserisce in nota una breve scheda, cui si rimanda nei successivi riferimenti.

«Passato e presente», a. XXXI (2013), n. 88 
carico la sicurezza di Allende, creato subito dopo le elezioni del 4 settembre 1970, non confidando nelle Forze armate.

La composizione della fonte orale, attraverso la registrazione del racconto di vita generato dall'incontro tra storico e intervistato, avviene in forme diverse rispetto all'interrogazione puntuale del protagonista, alla mera intervista giornalistica o ad altri modelli di trascrizione di conversazione. Partire da tale narrazione dialogica per arrivare a trattare un episodio specifico vuol dire scalpellare la materia, come uno scultore fa con il marmo per arrivare a usare solo pochi frammenti del tutto ch'è l'intervista. Questa non verte sull'oggetto specifico sul quale poi s'articola un saggio come questo, ma si origina in un contesto più ampio dove si generano molteplici incontri: quello tra storico e intervistato, con le rispettive soggettività e visioni di mondo; tra il presente e il passato e la storia e il desiderio, ovvero la narrazione del passato come viene raccontato e come avrebbe potuto essere. Così emergono inquietudini portate dentro una vita, problemi politici sottaciuti, la relazione tra intervistato e potere, domande che non hanno trovato risposta o che non sono mai state poste. Il prodotto, spesso raggiunto attraverso molteplici incontri e molte ore di registrazione in luoghi come la casa dell'intervistato, che mettano quest'ultimo in una condizione di agio, viene poi in gran parte eliso. A volte appena un frammento viene utilizzato. Ma da quanto elide lo storico s'arricchisce, potendo trattare il tema specifico partendo dal vissuto - precedente e successivo - della fonte. Credibile o meno, come qualunque altra fonte, oltre agli avvenimenti ci restituisce così anche il significato che i fatti hanno per le soggettività che contribuiscono con lo storico alla composizione del documento orale. Il resto (l'incontro, l'empatia, le incertezze, l'espressività, le emozioni) permette l'individuazione, la comprensione e l'interpretazione di quel frammento, usando la soggettività stessa come strumento di analisi.

Quelle escusse per il saggio in questione sono fonti peculiari nell'ambito della riflessione sulla storia orale. In quanto allendiste, sconfitte dalla storia, messe a tacere nei decenni successivi, imprigionate, esiliate, disprezzate e, con due eccezioni, appartenenti a classi subalterne, rispondono ai canoni della «restituzione di voce» ch'è uno degli obiettivi metodologici primari della storia orale. La loro narrazione è antitetica a quella della dittatura militare ma si discosta anche dalla ricostruzione dominante di parte democratica, così come si è sedimentata nel corso del tempo. Tuttavia, essendo anche nella posizione testimoniale dell'aver vissuto un evento capitale della storia del XX secolo, tale subalternità diviene peculiare, altera, alterità piuttosto. La storiografia non restituisce loro voce ma si giova di questa, partendo da tale alterità sociale, economica e politica rispetto a la historia oficial, per raffinare il quadro interpretativo degli eventi stessi che segnarono la fine del tentativo di realizzare il socialismo per via parlamentare.

Pur concentrandomi sulle fonti interne al campo democratico, nella peculiarità della transizione cilena dopo 17 anni di dittatura militare e un ventennio di democrazia che mantiene intatta l'impalcatura pinochetista dello Stato, tali voci ci restituiscono un quadro dicotomico e quasi manicheo tra differenti 
rappresentazioni degli eventi del periodo di Unidad Popular, del colpo di Stato e della dittatura. Da una parte vi è la narrazione dei socialisti riformati, che si riconoscono nei governi della Concertazione imperniata sull'alleanza tra Partito socialista e Democrazia cristiana. A tale ricostruzione ci si riferisce indirettamente nel testo quando vi fanno riferimento le fonti utilizzate. Dall'altra parte vi sono quelli che possiamo definire allendisti puri e che si rifanno anche umanamente all'eredità dell'esperienza del governo popolare. All'interno di quest'ultimo sottogruppo si collocano le fonti utilizzate. Queste a loro volta ci offrono due possibili evoluzioni di cosa successe all'interno del Palazzo della Moneda l'11 settembre. Una è quella dei collaboratori strettissimi di Allende, in particolare il ministro della Sanità Arturo Jirón ${ }^{4}$ e l'addetto stampa Carlos Jorquera ${ }^{5}$. Sono persone di grande rispettabilità: un famoso giornalista televisivo e il miglior chirurgo del paese ${ }^{6}$. Hanno mantenuto intatti i loro principi allendisti, guardano con pacificato disgusto alla storia cilena post-73 e post-89, ma hanno risolto i loro conti con la storia e sentono che il loro punto di vista, a lungo negato, è finalmente riconosciuto. I Gap, che ci offrono la seconda narrazione degli stessi accadimenti, all'epoca erano giovani militanti socialisti. Oltre che della memoria dei compagni caduti e della lealtà al presidente, si considerano invece custodi di verità scomode, come l'epopea negata della «battaglia della Moneda», quando avrebbero tenuto testa per molte ore all'intero esercito cileno, per antonomasia il meglio armato e addestrato del continente ${ }^{7}$.

La fonte orale dei sopravvissuti della Moneda, alla luce del «futuro anteriore» che li aspettava all'indomani del golpe, diviene fondamento interpretativo di un passato che per noi si arricchisce di ulteriori elementi di approfondimento. I documenti utilizzati non ci offrono già più o non solo la narrazione di quanto in sé sarebbe avvenuto, ma anche l'opportunità di riflettere su come quei fatti siano stati raccontati nel corso degli anni e come, in particolare la pubblicistica, si sia spesso adattata alle contingenze politiche. Così il documento intervista s'inserisce, contrappunta e in alcuni casi scardina narrazioni consolidate. L'ha sempre fatto rispetto alla versione falsa dell'assassinio del presidente, pur a lungo egemone. Continua a farlo problematizzando più che confutando - la rappresentazione del suicidio così com'è passata nel corso degli anni tesa a semplificare, espungere, alcune possibili interpretazioni del gesto che mise fine alla vita di Salvador Allende, la cui immagine è stata sistematicamente ferita da diffamazioni e irrisioni anche rispetto al senso alto della sua morte.

${ }^{4}$ Arturo Jirón Vargas, 1930. Medico, ministro della Sanità sino a fine agosto del 1973. Dopo il golpe è internato nel campo di prigionia dell'isola di Dawson, nell'Antartico cileno, quindi esiliato in Venezuela.

${ }_{5}^{5}$ Carlos Jorquera Tolosa, 1924. Giornalista televisivo, amico personale e capo ufficio stampa di Salvador Allende. Dopo il golpe è internato a Dawson, esiliato in Venezuela.

${ }^{6}$ Ó. Soto, El último día cit., p. 144.

${ }^{7}$ Cfr. P. Manns, Chile: una dictadura militar permanente. 1811-1999, Sudamericana, Santiago 1999; M.R. Stabili, Il Cile cit., pp. 49-57, 181-86. 
Così la specificità esperienziale dei testimoni, in particolare i Gap, rispetto ad attori e ricostruzioni quali quelle offerte dalla stampa e dall'interno del Partito socialista, che non considerano terzi ma interni alla loro storia personale, è conflittuale:

Nella maggioranza delle testimonianze - afferma Manuel Cortés ${ }^{8}$ - si parla del Gap ma in pochi sanno chi eravamo e quanti eravamo ${ }^{9}$. Lo stesso partito socialista non vuol sapere nulla di noi. Noi siamo quella parte della storia che per loro... magari si potesse cancellare. Ed è perciò che ogni volta che apriamo bocca ci tocca metterci in contraddizione con tutto quello che la gente ha scritto.

Helena Aranera Valderrama ${ }^{10}$ sostiene: «i Gap, noi che lavoravamo con il presidente, mai nella vita daremo una testimonianza falsa. Per lealtà. Perché stiamo parlando della vita dei compagni. Della vita del compagno presidente. E dare una falsa testimonianza... mai». Nell'affermazione di Helena vi sono tre aspetti ben noti a chi lavora con fonti orali, in particolare in contesti dove la fonte è sopravvissuta rispetto ad altri, vittime o caduti, ai quali è legata da una forte dinamica di appartenenza. Il primo è un auto-conferimento di autorevolezza, per il quale il testimone ${ }^{11}$ assegna alla propria narrazione un valore in sé che non riconosce ai non presenti. Il secondo punto è che le fonti si sentono sciolte da ogni interesse di parte ${ }^{12}$ per sentirsi invece legate, ed è il terzo aspetto, alla lealtà verso i compagni caduti. Può essere un esercizio retorico, che non esclude aporie della memoria in buona o cattiva fede, ma va considerata la conflittualità necessaria verso l'istituzione - il partito - intorno alla quale è girata la loro esistenza, e la forza etica che in tali fonti lega il passato al presente. I sopravvissuti si sentono obbligati a vivere la vita rappresentando i caduti; un peso, ma anche - ritengono - una legittimazione.

Una volta tratteggiati i caratteri dell'esperienza del governo di Unidad Popular ritengo necessario collocare le fonti attraverso la voce delle stesse. Vi emergono alcuni caratteri autoidealizzanti tanto spesso studiati sia rispetto alla narrazione del proprio passato, sia rispetto alla storia del Cile. Quindi è utile toccare, alla luce dell'esperienza specifica della maggior parte degli intervistati d'aver difeso in armi il presidente, il problema scomodo e poco si-

${ }^{8}$ Manuel Cortés, 1942. Socialista, membro dell'Eln (Esercito di liberazione nazionale che svolgeva funzioni di appoggio per il Partito socialista alla guerriglia del Che in Bolivia), fondatore dei Gap. Combatte la battaglia della Moneda dal ministero dei Lavori pubblici; esiliato, partecipa alla liberazione del Nicaragua.

9 Ó. Soto, El último día cit., pp. 249-52. In una delle più accurate ricostruzioni dell'11, che metodicamente elenca i presenti, l'autore stesso ammette di avere solo un'idea vaga sui Gap presenti.

${ }^{10}$ Helena Aranera Valderrama, 1944. Aiutante della madre, Rosa Valderrama, domestica di casa Allende, subisce il bombardamento della residenza di Tomás Moro, clandestina, si vede per anni rifiutato il lavoro.

${ }^{11}$ Cfr. la prefazione di P. Levi, I sommersi e i salvati, Einaudi, Torino 1986.

12 S. Portelli, La memoria e l'evento. L'assassinio di Luigi Trastulli, «Segno critico», 4 (1980), pp. 115-42. 
stematizzato dalla storiografia della (mancata) difesa armata del Cile popolare. Solo dopo aver dato profondità di campo alle nostre fonti possiamo giungere all' 11 settembre visto dall'interno del Palazzo della Moneda, per riflettere sulle narrazioni della battaglia e infine giungere al racconto del suicidio del presidente. Nello specifico, in particolare la testimonianza del Gap Pablo Zepeda, ci permette di ragionare sul problematico crinale che porta, nei quasi quarant'anni trascorsi, dal considerare inizialmente l'assassinio del presidente come metafora politicamente utile della barbarie del golpe, al ritenere infine la rappresentazione del suicidio come atto di coerenza politica sia come quella più coerente con i fatti, sia come quella più utile ad interpretarli dal presente concertazionista. È uno spunto per riflettere su un'altra battaglia, quella per l'eredità di Salvador Allende nel Cile attuale.

\section{Quando il popolo leggeva Dostoevskij}

Salvador Allende era giunto al governo con la vittoria elettorale del 4 settembre 1970. Nel 1939, ad appena 31 anni, era stato ministro della Sanità nel breve governo del Fronte popolare di Pedro Aguirre Cerda. Dal 1952 aveva accumulato forze candidandosi per quattro volte alla presidenza, fino a vincere nel '70. La coalizione dell'Unidad Popular (UP) era formata dai partiti socialista, comunista, radicale, dal Mapu (sinistra democristiana), tenendo dialetticamente sotto controllo il Mir (movimento sinistra rivoluzionaria) tentato dalla lotta armata. È uno schema politico che ricalca quello dei fronti popolari degli anni '30 ed è un modello unico in un'America latina orientata in quegli anni dal trionfo cubano e dal fochismo guevarista. UP propugnava una «via cilena al socialismo» che operasse una rivoluzione all'interno di un cammino istituzionale, minimizzando il ruolo della violenza ${ }^{13}$.

Nel corso dei tre anni di governo di Unidad Popular fattori tanto endogeni come esogeni contribuirono a determinare l'esito finale nel colpo di stato ${ }^{14}$. Questi ultimi sono ampiamente studiati e risiedono innanzitutto nel ruolo svolto dalle imprese multinazionali, in particolare dell'estrazione del rame nazionalizzate da Allende, e nell'ingerenza statunitense. Più complesse sono le cause endogene del crollo di un regime democratico tra i più stabili del continente. Tra queste vi fu la sottovalutazione e il fallimento del controllo dell'eversione militare. Il governo Allende inizia e si chiosa con due eventi drammatici che concernono le forze armate. Pochi giorni dopo essere stato eletto, l'assassinio del generale costituzionalista e capo di Stato maggiore

${ }^{13}$ S. Allende, El pensamiento politico de Salvador Allende, Quimantú, Santiago 1971; J.A. Vieira Gallo, Transición al socialismo y experiencia chilena, Cesoc, Santiago 1972.

14 M.R. Stabili, Il Cile. Dalla Repubblica liberale al dopo Pinochet (1861-1990), Giunti, Firenze 1991; J. Haslam, The Nixon administration and the death of Allende's Chile, Verso, Londra-New York 2005; J.E. Garcés, Democrazia e controrivoluzione in Cile, Saggiatore, Milano 1977 (ed. or. Democracia y contra-revolución. El problema chileno, Buenos Aires 1974). 
René Schneider, da parte dei neofascisti di Patria e Libertad con l'appoggio di elementi dell'esercito e della Cia, volto a evitare che Allende entrasse in carica, dimostrò che l'eversione interna e internazionale non avrebbe dato tregua. Il 23 agosto 1973 le dimissioni di Carlos Prats (assassinato l'anno successivo a Buenos Aires nell'ambito del Piano Condor ${ }^{15}$ ), da pochi giorni ministro della Difesa oltre che capo di Stato maggiore, costantemente pressato, minacciato, oltraggiato dai suoi sottoposti in quanto generale costituzionale, lasciò campo libero al traditore Augusto Pinochet designato successore dallo stesso Prats. Sul merito Joan Garcés parlò di doppio stato ${ }^{16}$ e il caso cileno fece a lungo riflettere in Italia Enrico Berlinguer ${ }^{17}$ ed ebbe un ruolo esplicito nel far maturare l'idea di compromesso storico con la Democrazia cristiana di Aldo Moro. Sul fronte interno Allende poté contare soprattutto sulla disciplina del Partito comunista. Le spinte dall'interno del suo partito alla radicalizzazione dello scontro risultarono invece un grave fattore di destabilizzazione. Nonostante tutto, in tre anni, il governo Allende realizzò la nazionalizzazione del rame e la maggior parte del programma cantato in campagna elettorale da Víctor Jara. Mantenne inoltre dati macroeconomici positivi nonostante l'inflazione e accumulò forze passando dal 36,6\% del settembre 1970 al 43,5\% nelle elezioni parlamentari del febbraio 1973. Nelle piazze, destra e sinistra si confrontarono a viso aperto, ma di più poterono le trame nere, l'aggiotaggio di beni di prima necessità $\mathrm{o}$, sul fronte opposto, l'occupazione di terreni appoggiata dagli elementi più radicali della maggioranza e dal Mir che contribuirono a spaventare le classi medie e polarizzare la situazione. Le parole di fuoco, in particolare quelle del segretario socialista Carlos Altamirano, gli slogan echeggianti una rivoluzione armata, restarono però tali. Fu chiaro l'11 settembre quando quella violenza, spesso evocata anche dal campo popolare, risultò essere a senso unico.

«Io - Jorquera - sono sempre stato un uomo di sinistra, ma non sono mai stato iscritto a nessun partito. [...] amico del Chicho ${ }^{18}$ [soprannome di Allende] già è un'onorificenza, e inoltre... allendista nelle quattro campagne [elettorali], dal '52 fino al '70». Anche Arturo Jirón si schermisce:

15 S. Calloni, Operación Cóndor: pacto criminal, La Jornada, México D.F. 2006; F. Martorell, Operación Cóndor. El vuelo de la muerte: la coordinación represiva en el Cono Sur, Lom Ediciones, Santiago 1999; N. Mariano, Operación Cóndor: terrorismo de estado en el Cono Sur, Lohlé-Lumen, Buenos Aires 1998.

16 J.E. Garcés, El Estado y los problemas tácticos del gobierno de Allende, Siglo XXI, Città del Messico 1973.

17 R. Nocera-C. Rolle (a cura di), Settantatré. Cile e Italia, destini incrociati, Think Thanks, Napoli 2010; G. Carletti, La sindrome cilena. Il Cile di Allende e la sua influenza sulla stampa di sinistra in Italia, tesi di laurea, Facoltà di Scienze della comunicazione, Università di Macerata, a.a. 2003-04; A. Mulas, Allende e Berlinguer: il Cile dell'Unidad Popular e il compromesso storico, Manni, Lecce 2005; M. Galeazzi, Il Pci e il movimento dei paesi non allineati, FrancoAngeli, Milano 2011, pp. 235-48.

18 C. Jorquera, El Chicho Allende, Ediciones Bat, Santiago 1990. 
Mio padre [il senatore radicale Gustavo] era molto amico di Salvador Allende. [Quando] mi chiamarono [...] ero un professionista con una carriera accademica e assistenziale, importante, no? Per me era un onore essere vicino a un uomo che appoggiavo politicamente e vicinissimo alle mie idee [...] economicamente fu un disastro. Dedicavamo al presidente il tempo della clientela privata e ovviamente non eravamo pagati. Così che economicamente non mi è mai andata male come in quell'epoca. Ma probabilmente non sono mai stato così felice.

I Gap si raccontano come un proletariato cosciente di sé, che viene da lontano e si mostra ancora sicuro di andar lontano. Renato González ${ }^{19}$ è di San Antonio, sulla costa pacifica: «la mia origine è in una famiglia operaia, umile. [Però] ho l'onore - tiene a considerarsi aristocrazia operaia - che mio nonno fu il fondatore del partito socialista nella provincia di Rancagua: Don Juan González». Manuel Cortés, detto Patán (il casinista):

Ho studiato dai salesiani una professione. Sono tecnico elettricista ma non ho mai lavorato come tale per le mie grandi inquietudini sociali. La mia prima militanza fu nell'Eln cileno, Esercito di Liberazione Nazionale, la parte del partito socialista che lavorava con la guerriglia del Che in Bolivia [diretta da Elmo Catalán, socialista e capo ufficio stampa di Allende nella campagna elettorale del 1962, caduto a Cochabamba nel 1970]. Così arrivai fin dall'inizio alla sicurezza personale del presidente. Eravamo i primi: cinque socialisti e cinque del Mir.

Pablo Zepeda20 è figlio di piccoli commercianti del nord: «nel $1973 \mathrm{mi}$ chiamarono per un corso e alla fine fui accettato come scorta. E l'11 settembre ero insieme al presidente nel palazzo della Moneda». Se i ricordi di Mamma Rosa, ultranovantenne, storica domestica degli Allende, sono oramai solo aneddotici e familiari, è sua figlia Helena a puntualizzare: «Sono figlia naturale, mia madre si chiama Rosa Valderrama, mio padre credo che si chiamasse Luís. Tutta qui la famiglia e quindi mamma doveva lavorare». Umilissima è anche la provenienza di Jaime Hernández ${ }^{21}$, autista di Allende: «Sono nato e cresciuto nel Cimitero generale. Nel 1962, finito l'ottavo anno di scuola mi sono iscritto al partito socialista e ho cominciato a lavorare come seppellitore. Non avevo 15 anni e mi assegnarono alla fossa comune. Dovevo sistemare i corpi degli n.n.». Jorquera pensa che «la Rivoluzione cubana ci ha orientato più che altro dal punto di vista morale. Ho ancora in un mio altare

${ }^{19}$ Renato González, 1953. Membro del Gap, combatte nella Moneda ed è tra i tre sopravvissuti della scorta. Si rifugia in Messico e poi a Cuba, che da allora è il centro della sua vita.

${ }^{20}$ Pablo Zepeda, 1951. Membro del Gap, combatte nella Moneda ed è tra i tre unici sopravvissuti della scorta. Quindi espleta funzioni militari per il partito, soprattutto in Bolivia, dove sconta 3 anni di carcere su una condanna di 30. François Mitterrand paga un riscatto per evitare che sia consegnato a Pinochet. Dall' 86 al '98 in esilio a Parigi.

21 Jaime Hernández, 1947. Autista di Allende. L'11 consegna per i Gap armi nel cordone industriale, cade, orribilmente torturato, fugge. Clandestino nel cimitero, privato della cittadinanza cilena, va in esilio in Australia. 
intimo la figura del Che, che ho avuto l'onore di conoscere, per l'impronta etica con la quale ha orientato intere generazioni di giovani latinoamericani». «Alla morte del Che - Renato González - avevo 14 anni e sette giorni. Era una settimana che avevo preso la tessera della gioventù socialista». Ma la via cilena, la via di Allende era diversa, no? «La via armata per il rovesciamento del regime capitalista era forte anche da noi. $\mathrm{E}$ da lì nasce il Mir con il quale condivido amicizie, dibattiti, campeggi. E anche io come adolescente avevo fretta e le armi mi sembravano la via più lineare. Le cose cambiano con Allende... iniziano le discussioni con il Mir e io mi schiero con il presidente». Secondo Manuel: «noi avevamo un'idea chiara del nostro obiettivo e con l'arrivo al governo dell'Unidad popular si apre la possibilità di avanzare nell'istaurazione del socialismo in Cile. [...] Era un progetto di transizione, ma per la prima volta la distribuzione della ricchezza cominciava a essere più equanime». Per Jirón «con il trionfo di UP il popolo alzò la testa [...] la gente leggeva... Dostoevskij! E la cosa più bella era vedere un popolo intero che si svegliava ed esigeva di accedere alla cultura». Il partito di Allende incarna anche per Milton Silva ${ }^{22}$ un'accelerazione:

Io vengo da una famiglia comunista. Mi avvicino al socialismo perché il partito comunista era troppo lento. In quegli anni non avevamo la televisione... tutto succedeva per strada. Anche se uno era scettico poi vedeva realizzarsi il programma: le relazioni con Cuba, col Vietnam, la nazionalizzazione del rame e della banca privata, la riforma agraria. [...] Indipendentemente dagli errori, dai dirigenti, dai sabotaggi, dall'embargo statunitense, dalla disinformazione, l'aggiotaggio... a marzo del ' 73 superammo il $46 \%$ dei voti. Perché le masse erano allendiste, il popolo appoggiava il governo e voleva che il processo continuasse. Così la destra arriva al golpe perché aveva fallito in ogni altro tentativo.

Se Milton osserva la crescita del consenso popolare, l'occhio di Arturo Jirón guarda alla classe media:

Da fine '72 la società cilena si spacca. Io vivevo a Ñuñoa, un quartiere di classe media [a oriente del centro, verso la Cordigliera] dove l'opposizione era poca ma in ospedale e all'università le riunioni che prima facevamo in un grande auditorium alla fine le facevamo nel mio studio. [...] La campagna di terrore della destra era ben condotta. Miei colleghi medici erano convinti di perdere il loro benessere. Era una campagna primitiva... ti leveranno la seconda macchina... ti metteranno qualcuno in casa... ma era efficace. E alcune cose... le occupazioni di terreni, slogan come "avanzare senza trattare", la propaganda armata del Mir, danneggiarono. Così la classe media, che prima appoggiava Allende, fu spaventata talmente che poi appoggiò il golpe. Io ho avuto minacce, una bomba in casa e vi furono rotture dolorose, anche con un mio fratello.

${ }^{22}$ Milton Silva, 1951. Impiegato postale, socialista, entra nel Gap nel 1972 per le sue capacità atletiche e diventa istruttore. L'11 combatte in Tomás Moro. 
Nonostante le molteplici informazioni sull'imminenza del golpe, il Cile continua in quella «condotta autoidealizzante» descritta da Moulián ${ }^{23}$, per la quale rifiuta di prendere atto dell'irreparabilità della violenza nell' aria, il piccolo cabotaggio della propaganda armata del Mir, l'eversione attiva fin dal caso Schneider e alimentata da Henry Kissinger, i neofascisti di Patria y Libertad, quella solo evocata dal segretario socialista Carlos Altamirano. Per Carlos Jorquera: «mai avremmo immaginato che in Cile potesse succedere. Ricordo gente molto seria e benintenzionata che ancora poche ore prima del golpe diceva: "l'esercito cileno non sparerà mai contro il popolo"».

In questo contesto si erge la figura di Allende. Jirón ricorda che «molte volte nell'intimità lui mi diceva "io sono presidente per sei anni. Poi ci saranno elezioni. E se perdiamo andremo a casa". Il suo era un concetto di socialismo democratico, il che non implicava rinunciare a fare profondi cambiamenti in una società così arretrata». Rammenta Jorquera:

Così non si poteva andare avanti e il presidente decise per il plebiscito. È un fatto che agli avversari di UP non piace ricordare. Il plebiscito è un desaparecido tra i tanti. Fui io a scrivere il discorso che Salvador doveva leggere alla nazione [annunciando un referendum sul suo governo]. Lo scrivemmo in tre con il perro Olivares, il primo morto nella Moneda, e Joan Garcés. Il presidente doveva parlare lunedì 10 settembre ${ }^{24}$. Avevo preparato tutto... la scenografia... non trovavo la bandierina cilena che doveva stare sulla scrivania. Fuori c'erano già tutti i camion delle televisioni per trasmettere in diretta nazionale... allora bisognava spostare tanto materiale, non come ora... Era tutto pronto ma Salvador decide di rinviare al 12 su richiesta di Pinochet... problemi interni dell'esercito.

Oggi sappiamo che la richiesta di rinvio era parte della cospirazione.

\section{Il problema della difesa armata del Cile popolare}

Prima di giungere all' 11 settembre è utile sottolineare che uno studio sistematico sulla difesa armata del Cile di UP, che esula dai temi di questo saggio, resta ancora da fare, e non per caso. In dittatura prima e con i governi della Concertazione poi, il dibattito su tale tema è condizionato da considerazioni di opportunità, politiche, personali, giudiziarie che portano alla negazione di un fenomeno che concerne opzioni sconfitte, militarmente fallimentari e politicamente scomode. Se nell'ultimo lustro di dittatura il Partito comunista può rivendicare come di un qualche successo l'azione del proprio

23 T. Moulián, En la brecha. Derechos humanos, críticas y alternativas, Lom, Santiago 2002, pp. 90 ss.

${ }^{24}$ P. Verdugo, Salvador Allende. Como la casa blanca provocó su muerte, El Ateneo, Buenos Aires 2003, pp. 164-68 (trad. it. Salvador Allende. Anatomia di un complotto organizzato dalla Cia, Baldini Castoldi Dalai, Milano 2003). 
braccio armato, il «Frente Patriótico Manuel Rodríguez» (Fpmr), figlio della rivalutazione critica dell'assenza di una politica militare all'epoca di UP, tutto quanto successo prima - la minima resistenza al golpe, i sogni di rivincita armata, i tentativi fochisti del Mir negli anni successivi - fanno preferire l'oblio.

A giustificare tale negazione vi è in primo luogo la propaganda del nemico, goebblesianamente ripetuta ad libitum. «La destra - batte il pugno sul tavolo Carlos Jorquera - parlava di 35.000 soldati cubani armati nel paese! C'era il Plan Zeta, il fantasioso colpo di stato di Allende 25 ! Tutte menzogne per spaventare la classe media!». Già nel 1984 il generale Gustavo Leigh si vanta dell'invenzione del Plan Zeta e dal 1999 migliaia di documenti declassificati negli Usa confermano che l'intera operazione è parte della «guerra psicologica» contro il governo democratico ${ }^{26}$. Così nel Cile della paura prima e dell'impunità poi, negare l'esistenza di una difesa armata del governo popolare vuol dire non prestare il fianco alla propaganda ed evitare di riaprire ferite politiche. Eppure, al tempo di UP, di armi si parlava molto e a sproposito. Sia il Partito socialista, con il suo slogan «avanzare senza trattare», che il Mir («popolo, coscienza, fucile») evocavano lo scontro. Solo il Partito comunista, fermo nel suo «no alla guerra civile», rassicurava Allende. In merito le fonti orali ci restituiscono un frammento degli eventi, spesso sottovalutato. Per Manuel Cortés:

Nel periodo finale i nostri dirigenti non furono all'altezza. Altamirano fu un irresponsabile. I dirigenti militari del partito, quelli che io chiamo "generali della sconfitta", si sono nascosti o erano casualmente - fa il segno delle virgolette - all'estero. Javier Ulloa, che con la Concertazione è poi stato sindaco di Concepción, era il responsabile militare del partito... la mattina stessa del golpe si rifugia nell'ambasciata argentina. Lui era la persona che aveva in carico la difesa militare di tutto il paese!

«Il 9 di settembre - ricorda Helena Aranera Valderrama, in un contesto domestico - Don Salvador disse che Altamirano aveva svegliato il caimano». Jaime Hernández, che 1'11 consegna per i Gap armi nelle fabbriche Fensa e Madensa, grida ancora rabbioso: «uno dei grandi traditori della classe lavoratrice è il segretario generale del mio partito Carlos Altamirano. Lui era lì la mattina dell' $11[\ldots]$ fino al giorno prima era il più rivoluzionario di tutti, a

25 J. Haslam, The Nixon cit., p. 227. Identiche fandonie, 20.000 cubani armati e un autogolpe in arrivo, sono usati in Venezuela per giustificare il fallito golpe contro Hugo Chávez l'11 aprile 2002: cfr. G. Carotenuto, 11 settembre 30 anni dopo. Per uno studio comparato delle reazioni delle masse latinoamericane al golpismo contro governi popolari: dal caso di Perón, ad Allende a Chávez, «Zapruder», 1 (2003), n. 2, pp. 143-47.

26 «El Mercurio», che secondo il Senato statunitense (US Senate, Cover action in Chile 1963-1973, US Government, Washington 1975) riceve 1,5 milioni di dollari dalla Cia per destabilizzare il governo, e G. Vial, El libro blanco del cambio de gobierno en Chile, Lord Cochrane, Santiago 1973, sostengono che UP avesse un arsenale di 45.000 pistole, $12.000 \mathrm{AK}$ e 500 bazooka. 
parole voleva che si armasse il popolo e quando ci vede con le armi impedisce ai lavoratori di difendere il governo». La pretesa di armare il popolo è solo espressione di giochi di potere. «Loro - accusa Manuel Cortés - giocavano all'alta politica. Sostenevano di essere in grado di controllare una parte degli stati maggiori». Per Milton Silva si crea un corto circuito: «i militari costituzionalisti c'erano - fa un lungo elenco verificabile - ma stavano aspettando la risposta popolare. Che non ci fu perché i dirigenti che parlavano di rivoluzione e di armare il popolo lo facevano solo a parole». Per Raúl Blanchet, socialista infiltrato nell'esercito ${ }^{27}$ : «il partito preferiva non vedere i nostri rapporti sulla forza del golpe imminente, sulla crescita del terrorismo neofascista. [...] Preferiva non avere una politica militare puntando sull'idea che il golpe poteva essere arginato dalla spaccatura delle forze armate». Milton mescola memoria e desiderio: «Il 4 settembre [1973] ci fu una manifestazione enorme. Un milione di persone! Tutta questa massa voleva armi. Io mi domando cosa sarebbe successo se queste persone fossero state armate. Avremmo resistito? Sarebbe stato un massacro molto più grande? I golpisti vedendo la resistenza avrebbero ripiegato? I militari costituzionalisti avrebbero avuto la meglio?».

Il tema dell'addestramento all'estero di alcune centinaia di militanti e della reale entità dei presunti arsenali di UP sono tuttora difficili da trattare. Un militante della Sinistra cristiana (IC) mi dettaglia il proprio soggiorno in Cecoslovacchia dove casualmente incontra militanti socialisti del suo quartiere. Un altro testimonia di $150 \mathrm{AK}$ del Mir, nascosti a Concepción e non utilizzati. Piccole crepe, ma personaggi di primo piano come Jacques Chonchol, ministro della riforma agraria, mi negano con forza l'esistenza anche di un solo militante con addestramento militare. Chiosa Renato González: «nessuno può più negare che c'erano persone addestrate all'estero. E non è che ti potevi presentare in Cecoslovacchia individualmente. Dipendeva dai partiti». Jorquera puntualizza: «c'erano compagni molto cari che si facevano chiamare "comandante". Ma non avevano nulla a che vedere col governo». Anche sulle armi Manuel dettaglia:

All'inizio avevamo armamento americano regalato dai cubani, che è quello che si portò via il Mir. Dopo il '72 avevamo armi sovietiche, gli AK, gli RPG7 [anticarro], alcuni Dragonov. Successivamente, tramite il Banco Centrale, si fece un'importazione di armi che invece di andare per la sicurezza delle banche [alle quali era ufficialmente destinata] venne a noi. Così ci arrivarono le pistole Browning, le mitragliatrici Walter che usavamo per la scorta. Poi, per quello che ne so, il partito comunista aveva $300 \mathrm{AK}$ che non furono mai utilizzati, il partito socialista ne aveva 150 e altri 150 li avevamo noi del Gap. Quindi armi ce n'erano. Ma le uniche che furono distribuite furono l'eccedenza di Tomás Moro.

\footnotetext{
${ }^{27}$ Raúl Blanchet, 1951. Infiltrato nell'esercito dall'apparato d'informazione allendista.
} 
Anche dopo il golpe Manuel vive su di un ramo secco della storia del suo partito:

Dal 1974 il partito [socialista] decide, su richiesta di tutta la base, di avere una vera politica militare. [...] Non già per guerriglieri ma corsi in normali accademie militari. Tra socialisti e comunisti ci siamo diplomati in più di 500 cileni in tutte le specialità: truppa, fanteria, carristi, genio, ingegneri delle comunicazioni, radar, armamenti [...]. Nell'offensiva finale a Managua [1979] eravamo più di 180 ufficiali cileni. Io ero consigliere di Eden Pastora. Poi ho passato sette anni e mezzo aspettando e combattendo la Contra in Nicaragua [...] ma secondo i dirigenti in Cile non c'erano mai le condizioni. Solo nell' 87 riuscii a venire qui in missione. Era già cambiato tutto e l'uscita concertata dalla dittatura era già decisa. Già ad Ariccia ${ }^{28}$, in Italia, erano iniziate conversazioni e nell' 86 , sotto gli auspici di Felipe González, il partito socialista si riunisce alla Moncloa con [... i militari e] si decide la transizione, il plebiscito [dell'88] e l'impunità. E quando Pinochet viene arrestato a Londra [1998] i militari iniziano a reclamare il rispetto degli accordi. Nessuno nel partito socialista ha avuto il coraggio di rivelare che accordi firmarono!

Né autogolpe, né guerra civile, né lotta di liberazione. Nemmeno una struttura armata in grado di mettere in salvo decine di persone a rischio, che oggi allungano l'elenco dei desaparecidos. «Noi eravamo - ricorda tuttora vibrante Milton - un incipiente gruppo armato. Così sentii un'impotenza grande per non poter essere da tutte le parti, per non aver salvato vite... per non essere stato nella casa di Neruda. Nella casa di tanti compagni inermi che sono morti senza potersi difendere. E noi avremmo avuto la possibilità di farlo!».

Tirando le somme: i dirigenti, in particolare Altamirano, e il Mir evocavano un inesistente popolo in armi. Militanti addestrati all'estero e fallimentari apparati militari esistevano, ma l'11 non ebbero alcun ruolo e le poche armi restarono nascoste. Quanto esposto nella seconda metà di questo paragrafo conferma che una pur difficile ricognizione sulla difesa militare di UP e sulla lotta alla dittatura sia possibile ma resti scomoda per almeno tre motivi: la mancata salvaguardia delle istituzioni democratiche, la transizione patteggiata con i carnefici, l'eredità politica di Allende.

\section{La battaglia della Moneda?}

«Alle 6.30 - Renato González ha appena montato la guardia nella residenza presidenziale di Tomás Moro - arriva una telefonata per il presidente della

\footnotetext{
${ }^{28}$ Quello riportato è il pensiero e la storia di Manuel Cortés. Non sfugge, ma esula dai temi di questo saggio, la complessità del rinnovamento socialista e il ruolo del Psi, del Pci, dell'eurocomunismo e del compromesso storico. Cfr. T. Moulián, Sobre la teoría de la Renovación: notas introductorias, «Chile-América» 82-83 (1982) p. 14; P. Zaldivar, La vita politica in Cile e i suoi rapporti con l'Italia. 1960-1990, in R. Nocera-C. Rolle (a cura di), Settantatré cit., pp.
} $100 \mathrm{ss}$. 
Repubblica. [...] Commuto la chiamata e [...] chiede che immediatamente venga messa a disposizione la scorta per andare alla Moneda». Su Tomás Moro sono spostati una cinquantina di membri del Gap. Tra di loro c'è Milton Silva: «Bruno, il mio capo, stava partendo verso la Moneda. Era un gruppo di nove compagni. Mi avvicino e dico "vengo con voi". "No, tu resti qui". C'era una certa disciplina militare e quindi... sono tutti desaparecidos». Milton resta e combatte a Tomás Moro dove la battaglia e il bombardamento sono quasi duri come alla Moneda. Mamma Rosa assiste Hortensia Bussi, moglie di Allende. Sua figlia Helena s'improvvisa chirurga estraendo a mani nude le schegge di proiettili ai feriti.

«Non conoscevamo ancora - è Pablo Zepeda a parlare - le dimensioni della sollevazione. Allende entra dalla porta principale con il suo AK in mano, quello che gli aveva regalato Fidel. Sono le 7.30 e le strade si animano e si sentono grida di "Allende il popolo ti difende"». Manuel Cortés, el Patán, è all'Hotel Conquistador [a tre isolati dalla Moneda] con una delegazione di minatori del rame.

Dividevo la stanza con un compagno di Chuqui [Chuquicamata, la più grande miniera di rame a cielo aperto del mondo]. Da buon minatore si alzava alle cinque di mattina. Alle sette torna e mi racconta che le strade sono piene di militari. Arriviamo alla Moneda prima delle 8, entriamo dal garage e mi ordinano di farmi carico delle auto, pronte per partire e di formare una squadra con gli autisti. Meno di dieci minuti dopo comincia la sparatoria.

Uscii di casa - racconta Jorquera - qui nella Piazza Ñuñoa [dove si svolge l'intervista], in macchina. Era un Fiat 1500, una gran auto per quell'epoca. C'era un traffico insolito tanto che chiesi a un capitano dei carabinieri. Mi dice che è meglio non andare. Mi conosceva come vecchio giornalista ma io insisto: "devo andare, è il mio lavoro". Mi risponde, non lo dimenticherò mai: "sotto la sua responsabilità, signor Jorquera".

Óscar Soto ${ }^{29}$ elenca le persone presenti nel palazzo intorno alle 8 di mattina, 84 più 17 detective (poliziotti) che non nomina. Sono membri del governo (17), giornalisti e consiglieri (14), medici (8 più Jirón), familiari e segreteria (12), forze armate (7) e i Gap (26, ma Soto ammette l'approssimazione). Alle 11 di mattina è già uscito il personale non politico, Joan Garcés, cittadino spagnolo, i militari salvo cinque poliziotti, le donne, comprese le figlie Isabel e Beatriz. Fa eccezione Miriam Contreras, la Payita, il grande amore di Allende. «Restano - ricorda il dottor Jirón - una ventina che difendevano la Moneda e una ventina di consiglieri molto vicini al presidente». I primi si muovono liberamente, gli altri sono rifugiati in basso a causa del bombardamento e, con poche eccezioni, tra le quali Jirón, non hanno più accesso al presidente. Appartengono a classi sociali diverse, vivono esperienze diverse, avranno un destino diverso. Dei primi sopravvivono in tre, «Pablo Zepeda,

${ }^{29}$ Ó. Soto, El último día cit., pp. 249-52. 
Juan Noces e io [Renato González]. Gli altri sono eliminati sotto la gestione del Generale [Javier] Palacio. Dalla Moneda sono usciti vivi, io stesso li ho visti e ci sono molti testimoni. [...] Sono stati legati con filo spinato, torturati e assassinati». Del secondo gruppo si salva la maggioranza, pur passando da prigionia ed esilio. Dal racconto di vita di cinque di loro (Jirón, Jorquera, González, Zepeda e Cortés) ci confrontiamo con quanto avviene quella mattina a Santiago. I politici, a lungo ammassati in un sottoscala, non parlano di battaglia. «C'era un gruppo che organizzò la difesa. Noi non avevamo accesso a loro... eravamo abbastanza inetti per difenderci da un attacco» ricorda Jirón. «Io avevo un revolver - parla Jorquera - assolutamente legale. Non ho sparato neanche una pallottola. A chi dovevo sparare? Dov'è... "la battaglia della Moneda"? Io credo che le battaglie siano un'altra cosa». Jirón ha più libertà di movimento: «mi passano una mitragliatrice. La prendo e... realmente era troppo pesante, "che ci faccio io?" Più tardi, al momento del bombardamento, mi passano un casco di acciaio. Ma era enorme... solo [già in prigionia] ho saputo che i militari usano due caschi, uno interno per la testa e quello d'acciaio fuori. Io non ne avevo idea».

Dopo una prima riunione e una serie di chiamate - rammenta Renato González Allende chiede di restare solo con noi Gap. S'informa delle armi e ci descrive la situazione: "questo è un golpe ed è coinvolta la maggior parte dell'esercito. Io resto alla Moneda per una decisione politica. Chi non è d'accordo è libero di uscire perché qui dentro siamo in trappola. Può portare le armi e appoggiarci da fuori”. Insomma... in nessun momento fummo ingannati. [...] Lì ti rendi conto della dimensione reale... avevamo un impegno morale che era la difesa del dirigente e da un momento all'altro la cosa diventa esorbitante. Non sei più la scorta, stai difendendo l'intero processo rivoluzionario. Non difendevamo più una persona ma un simbolo. E non ci fu neanche bisogno di guardarci in faccia: restammo tutti. E per scorno dell'esercito cileno eravamo sedici!

Jirón ricorda che «arrivavano le chiamate, inizialmente [Allende] era preoccupato per Pinochet. Ci furono dialoghi forti con i golpisti». Pablo Zepeda si è già levato la paura della battaglia: «appena arrivo al Palazzo devo correre in bagno. Dopo di quel momento... una cosa normale». Anche Renato, racconta un'esperienza simile: «Nel preambolo avemmo molta paura. Chi dice che non ha paura è un tremendo bugiardo. Quando per la prima volta ci affacciammo dal secondo piano e vediamo carri armati, cannoni, jeep, migliaia di uomini, dicemmo: "qui resistiamo 10 minuti"». «E invece se non fosse stato per l'incendio - segue Manuel Cortés - potevamo continuare. E se avessimo avuto le conoscenze che abbiamo ora dell'arte militare avremmo resistito tre settimane». Forse Patán è un po' miles gloriosus, ma è difficile contestare che «abbiamo resistito otto ore. [...] Un esercito come quello cileno avrebbe dovuto prendere la Moneda in dieci minuti. E invece non sono riusciti a entrare. Hanno dovuto usare i carri armati, l'aviazione, le bombe incendiarie...». 
Marco Antonio de la Parra definisce 1'11 «un giorno pieno di radio»30. La cifra di Arturo Jirón è l'understatement per l'esperienza personale («il presidente mi diceva sempre che io ero politicamente un ignaro»), accompagnata dall'ebbrezza di aver vissuto una vicenda che sa straordinaria: «io ero di fronte al presidente durante il discorso a Radio Magallanes ${ }^{31}$. Ero seduto in un sofà di fronte alla scrivania, dove lui faceva, accovacciato, il suo discorso delle grandi alamedas». Jorquera, da comunicatore, sottolinea che «non è facile dire a braccio quello che Allende disse. Viveva una sorta di trance storico ed ha tenuto un discorso, quello delle "grandi alamedas dove passa l'uomo libero" che resterà come uno dei più grandi discorsi politici nella storia dell'umanità». Arturo ribadisce: «ero impressionato [...] non pensavo alla mia situazione personale. Mi sentivo davvero di fronte alla storia e a una figura di portata straordinaria. Sentivo una profonda ammirazione per un uomo che per aver cercato di cambiare questo paese era praticamente condannato a morte. E lui sapeva che sarebbe morto lì».

«Poco prima del bombardamento mi prese a parte: "monito [scimmietta, è il soprannome di Pablo Zepeda], perché non te ne vai, sei troppo giovane"». Si rifiuta. «Lui mi abbracciò e ritornammo alla battaglia. Poco dopo mi chiese di farmi carico del telefono. Io ricevo la chiamata di Augusto Pinochet. Il presidente mi fece rispondere che non voleva parlare con un vigliacco, fellone e traditore». Alle 11 Augusto Olivares, il direttore della televisione, si toglie la vita. «Col perro eravamo come fratelli. Mi è scappato via. È un ricordo che è puro pianto, gridai, arrivarono Allende, Jirón... ma il perro stava...» la frase di Jorquera si perde in un sospiro. A Pablo il presidente si mostra in tutta la sua fragilità: «Il presidente mi prese a parte e in un corridoio dal lato di Morandé mi afferrò, mise la testa sulla mia spalla e si mise a piangere. Quindi si riprese, tornammo indietro e fece fare un minuto di silenzio per il compagno Olivares. Aspettavamo già il bombardamento».

Nessun partito di Unidad Popular - scandisce le parole Renato González, mentre guardiamo l'oceano dal porto di San Antonio in una gelida mattina australe - ha compiuto il proprio dovere di fronte alla storia. Noi Gap sapevamo che arrivava lo scontro ed eravamo pronti. Organizzammo la difesa della Moneda contemplando il ruolo dei partiti. Era un piano concordato tra i Gap e i responsabili militari di ogni partito. Non hanno rispettato i patti e ci hanno lasciati soli. E siamo ancora in attesa di una spiegazione.

Qual era il piano?

Il Gap difendeva il palazzo. Non era necessario ci fosse più gente. All'esterno ogni partito doveva presidiare un edificio. E invece non si presentano! [...] Arrivò un

${ }^{30}$ M. Rivas-R. Merino (a cura di), Qué hacía yo el 11 de septiembre de 1973?, Lom, Santiago 1997, pp. 65-70.

${ }^{31}$ El último discurso de Salvador Allende, in www.youtube.com/watch?v=g1QJ-y_xUmk>. 
solo compagno, e la storia lo salva: Mario Melo Panadera, il "pelato Melo". Militante socialista, ex-mirista. Non arrivò né il Mir, che era il gruppo ultrà, non arrivarono i socialisti, non arrivarono quelli del Mapu, i radicali, i comunisti. L'11 settembre ci siamo ritrovati soli dentro la Moneda. Di fronte alla storia, i partiti ne dovranno rispondere.

\section{L'unico appoggio esterno viene dagli uomini di Manuel Cortés:}

Eravamo in otto, io, sei autisti e il ragazzo di Chuqui. Saliamo al secondo piano del ministero dei lavori pubblici. Avevamo AK, un RPG7 e una mitragliatrice "punto 30 ", di quelle americane con un aiutante che regge il nastro. Facevamo avanzare i militari e aprivamo il fuoco. Li respingiamo 4-5 volte finché non cambiano tattica. Mandano un carro armato e un franco tiratore che ferisce uno dei nostri. Lì siamo noi a variare finché il negro David non obbliga il franco tiratore a ritirarsi. [...] Se avessi avuto le conoscenze che ho ora... abbiamo commesso un errore grande a metterci al secondo piano. Dovevamo stare tutti al primo piano e con quattro mitragliatrici ai quattro angoli, col tiro radente - fa il segno col braccio come a spazzar via il nemico - avremmo potuto resistere per giorni. Invece io stavo con la punto 30 al terzo e poi al quarto piano! Questo si vede nei film americani ma non serve a nulla!

Per Renato González l'azione dal ministero è la conferma che il piano poteva funzionare. E una parte di questo assume contorni politici rilevanti: «tra le alternative previste c'era l'evacuazione del presidente dalla Moneda ${ }^{32}$. C'è un momento nel quale Allende domanda "com'è la situazione fuori?"». È un dettaglio nel quale il racconto di Renato differisce da quello del Patán: «alle 10 mandai Lalo perché c'era la possibilità di fare uscire Allende verso il Ministero dei lavori pubblici. Avevo fatto delle verifiche e in quel momento era possibile evacuarlo secondo i piani. Lalo tornò dopo 20 minuti. S'era avvicinato ad Allende ma questo l'aveva redarguito: "compagno, torni al suo posto di combattimento". E lui non aveva avuto il coraggio». Riprendiamo il racconto di Renato:

Purtroppo eravamo bloccati. I compagni nel ministero stavano impedendo a due carri armati di entrare da Morandé. Il presidente avrebbe dovuto attraversare la strada ed entrare nei garage. Da lì nelle cantine potevamo portarlo verso una banca con uscita sull'Alameda. E da lì nasconderlo in un luogo leale. E questa è la prova che se i partiti fossero stati ai patti le cose potevano andare diversamente: i compagni del ministero hanno tenuto un fuoco costante che ha sempre impedito al nemico di entrare per Morandé. Abbiamo resistito otto ore, potevamo arrivare alla notte, che ci avrebbe favorito.

Torniamo al Patán: «Alle 11 provo a parlare per telefono per fare un nuovo tentativo per fare uscire il presidente. Tra le cose che mi dissero dalla Moneda c'era che sarebbe arrivata dall'Alameda una colonna armata di 1.500 compagni del Mir». Ma se neanche esistevano 1.500 armi! «Certo che no.

\footnotetext{
32 Il dato mi è stato confermato dallo storico Mario Garcés, già quadro del Mir.
} 
Ma il Mir qualche arma aveva e 95 le avevano portate via a noi dei Gap, tra le quali alcuni cannoni da 57». La guerra e le false notizie, avrebbe chiosato Marc Bloch. Arriva il bombardamento. Jirón sostiene che si salvarono solo per una casualità: «Ci mettemmo in un corridoio nella parte orientale del palazzo. Gli aerei [che provenivano dalla cordigliera, quindi da Est] lanciavano le bombe sul centro del palazzo. Perciò la parte più distrutta è la metà occidentale e noi restammo vivi nella Moneda che bruciava per intero». Il racconto di Pablo Zepeda è affannoso, come se tornasse a mancargli il respiro:

Bombardavano dall'aria mentre dall'esterno, dalla Via Teatinos, ci tiravano lacrimogeni. Era irrespirabile, il caldo, le fiamme, un inferno. Il presidente ci faceva passare le maschere, ci faceva stare lunghi, in basso si respirava meglio. E appena passava il bombardamento riprendevamo le posizioni. Saliamo al secondo piano, già semidistrutto. Lì c'era la galleria con i busti dei presidenti. Ne facemmo una trincea. Il presidente [attivo nella difesa in tutti i racconti] ce ne fece rispettare solo uno.

Proviamo ad indagare ma nessun testimone è in grado di ricordare quale. Probabilmente era José Manuel Balmaceda o Pedro Aguirre Cerda. A Tomás Moulian, che forse non conosce il dettaglio del busto salvato, nella sua Conversazione ${ }^{33}$ piace immaginare comunque che lo spirito di Balmaceda, morto suicida nel 1891 in circostanze politiche non dissimili, accompagni Allende in quelle ore. I Gap al secondo piano sparano anche durante il bombardamento. «Secondo me - commenta Renato González - per ignoranza. Siamo stati i primi cittadini cileni della storia ad aver subito un bombardamento [...] quasi non si parlava. Si lavorava. Le parole non servivano. Se ci incrociavamo ci guardavamo, ci toccavamo con affetto la faccia. Uno sguardo, un gesto... andiamo bene, stiamo bene». Nella parte finale del combattimento i Gap si dividono. Otto, tra i quali Renato, restano sopra. Sono gli otto che continuano a combattere ben oltre la morte del presidente, fino alle 15. Sapranno solo una volta catturati. Gli altri invece, tra i quali Pablo Zepeda, sono col presidente sino alla fine.

\section{Il suicidio come atto di coerenza politica: una lunga gestazione}

Salvador Allende si toglie la vita intorno alle 14. Nonostante sei ore di battaglia, i golpisti non sono capaci di prendere il palazzo difeso da un pugno di uomini. La resa è inevitabile a causa dell'incendio. Si siede su un divano del suo studio. Appoggia sotto il mento la canna dell'AK47 regalatogli da Fidel Castro e con il quale nel corso della mattinata ha combattuto. Preme il grilletto.

Per i testimoni non vi è mai dubbio ma, nonostante ciò, nasce subito la leggenda dell'assassinio. Da Pablo Neruda alla vedova Hortensia Bussi, da

\footnotetext{
${ }^{33}$ T. Moulian, Conversación interrumpida con Allende, Lom, Santiago 1999, p. 30.
} 
Fidel Castro a Gabriel García Márquez ${ }^{34}$, che romanza un improbabile duello finale col Generale Palacio, tutti sentono come l'omicidio, meglio del suicidio, chiosi la barbarie del golpe. Solo nel 2005 José Quiroga, l'unico medico presente alla Moneda ad alimentare tesi alternative, ammette che «era più importante l'aspetto politico che il mondo credesse che Allende fosse stato ucciso dai militari» ${ }^{35}$. È una menzogna, come quella sull'inesistente esercito liberatore al comando del Generale Prats inventata da Radio Mosca. Può prosperare per l'iniziale assenza della voce dei testimoni, sterminati, imprigionati, in clandestinità, quindi minimizzate e per il fatto che - solo casualmente la verità coincidesse con quanto affermava la giunta militare. Ancora nel 2003 l'autorevole giornalista Faride Zerán può presentare quasi come uno scoop il punto di vista del dottor Jirón che considera il suicidio un gesto politico $^{36}$. Nel 2011, in occasione dell'inutile riesumazione della salma, per il quotidiano «El Mercurio» è meritevole di un titolo il fatto che la senatrice Isabel Allende affermasse della morte del padre: «la nostra convinzione è che il presidente Allende prese la decisione di morire come atto di coerenza politica $^{37}$ ». Vengono in mente suicidi politico-testimoniali come quello di Jan Palach a Praga o quello di Cich Quang Duc, il primo monaco buddista vietnamita datosi fuoco a Saigon. È il gesto «di un lottatore sociale, non di un eroe [...] senza vocazione a un martirio che accetta con dolore ${ }^{38}$ » come estremo segno di rispetto per le istituzioni democratiche. Tra le fonti qui utilizzate Arturo Jirón e Pablo Zepeda si dichiarano presenti. Raccontano due suicidi diversi che aprono le porte a difformità interpretative. Le parole di Isabel sul suicidio come atto di coerenza politica possono uscire rafforzate o indebolite a seconda di come se ne soppesino le differenze. Arturo Jirón:

La Moneda era quasi interamente bruciata. Quando è evidente che ogni resistenza è inutile il presidente ci riunisce e dice "ci consegniamo". Si fa una fila, scendevamo uno a uno. Io sono l'ultimo. Già i militari sono nella scala del primo piano. In quel momento si sente una raffica di mitraglietta molto vicina. Uno dei ragazzi del Gap, uno dei direttori, grida: "il presidente è morto". Un grido terribile. Mi giro e vedo il presidente seduto nel divano con il cranio fracassato. Lì mi sorreggono varie persone, perché ero molto turbato e mi rimettono in fila per... consegnarci.

34 G. García Márquez, A ruota libera 1974-1995, Mondadori, Milano 2003, pp. 15-17 (ed. or. Chile, el golpe y los gringos, «Alternativa», n. 1, Bogotà 1974). Un'inchiesta di ampia circolazione ma di infimo valore è R. Rojas, Estos mataron a Allende, Martinez Roca, Barcelona 1974. 08.

${ }^{35}$ E. Labarca, Salvador Allende: Biografia Sentimental, Catalonia, Santiago 2005, pp. 401-

${ }^{36}$ F. Zerán, Arturo Jirón: “el suicidio de Allende fue un acto político”, «Rocinante», 2003, n. 58 .

${ }^{37}$ Nuestra convicción es que el Presidente Allende tomó la decisión de morir como un gesto de coherencia, «El Mercurio», 24 maggio 2011.

38 T. Moulian, Conversación cit., pp. 21-30. 


\section{Pablo Zepeda:}

Continuammo a combattere fino all'una passata. A quel punto il secondo piano era irrespirabile. Stava bruciando tutto. Scendiamo di sotto. Il presidente si siede sul divano. Era circondato dalla scorta. Erano ancora presenti alcuni medici, sicuramente il dottor Bartulín. Si mette il suo fucile tra le ginocchia. L'AK... Se lo appoggia sotto il mento. Lo aveva già fatto due o tre volte. Nessuno gli fece troppo caso perché si sedeva sempre a pensare. Improvvisamente abbassa la mano al grilletto e parte una raffica. Tutto ciò io l'ho visto direttamente. Restammo gelati. Nessuno fino a quel momento aveva immaginato che volesse terminare la sua vita così.

Entrambe le versioni provengono da persone credibili, che hanno legato l'intera esistenza alla lealtà verso il presidente e che non hanno, soprattutto a distanza di molto tempo, alcun interesse d'inquinamento. Jirón condivide la sua versione col dottor Patricio Guijón col quale si ritrova nella dura prigionia antartica a Dawson. Quest'ultimo, sarebbe tornato indietro a raccogliere un ricordo di quel momento storico e così racconta in tutte le testimonianze, coeve e successive ed è ripreso da Soto ${ }^{39}$. Pablo Zepeda, sfuggito alla morte, inizia una lunga odissea che nell' 86 lo vede riscattato dalle carceri boliviane da François Mitterrand. In esilio a Parigi fino al 1998, parla per la prima volta a Madrid davanti a Baltasar Garzón nel 1999. La versione Jirón-Guijón prevale una volta tramontata la tesi dell'omicidio. Quella Zepeda contrasta con un consolidamento della narrazione della Moneda che prescinde dalla testimonianza dei Gap, citandoli a stento.

Le principali differenze tra le due versioni concernono la forma della comunicazione della resa e la presenza o meno di persone in compagnia di Allende. Per noi è importante comprendere la genesi di tali differenze, e il significato di queste sia dal punto di vista esistenziale delle fonti sia da quello politico. La versione Jirón, quella dell'Allende suicida solitario, comporta una ricerca di una compostezza (in qualche modo borghese) nel gesto estremo per il quale cerca privatezza. È una versione dove tutto sembra essere in ordine e dove Allende sembra avere il pieno controllo degli eventi. Il suicidio come gesto politico ne diviene logica conseguenza, inequivoca.

La versione Zepeda è più problematica. Subentra una logica di gruppo per intendere la quale ci aiuta Manuel Cortés: «Io non ero lì ma ho ascoltato tutte le versioni. Anche quella del dottor Guijón che dice che Allende era completamente solo. Il fatto è che Allende non restava mai solo senza scorta. Anche quando voleva parlare di qualcosa di riservato, mandava tutti fuori, ma noi rimanevamo. Allende non poteva restare solo... non aveva autorità sulla sua sicurezza». Revisionando la lunga intervista concessami da Arturo Jirón, questi, come anche Soto, considera scontata (e di per sé non registrata) la presenza dei Gap. Più difficile è dirimere la contraddizione nella decisione di arrendersi. «Allende - Pablo è fermissimo - non ha mai parlato di arrendersi. [...]

${ }^{39}$ O. Soto, El último día cit., pp. 92-98. 
Noi eravamo lì. Io ho tutti i dettagli qui. Dei nostri c'erano Jano, Raúl, Aníbal... del Pelato non mi ricordo. Sono tutti morti». La resa era nelle cose, l'incendio generalizzato e l'aria irrespirabile ed è improbabile che Allende dimenticasse otto dei suoi che continuano a combattere per oltre un'ora. Allende sente una responsabilità paterna verso i Gap che accompagna quella politica. Renato ha 19 anni, Pablo 22, più volte li ha invitati a mettersi in salvo. Forse nel suicidio raccontato da Pablo c'è anche una sorta di liberazione di quei ragazzi dalla responsabilità della vita del presidente: «siete liberi, provate a salvarvi». Ma quella raffica pubblica, soprattutto nella condizione di minorità testimoniale dei Gap, può anche far rientrare dalla finestra interpretazioni faticosamente fatte uscire dalla porta. Può essere reinterpretata e divenire d'impeto, casuale, disperata, perfino vile. È questa la differenza tra la fila di prigionieri che scendono le scale a mani alzate della quale fa parte Arturo Jirón, con Allende che si ricava un ultimo spazio di privatezza, e l'Allende raccontato da Pablo Zepeda, che si uccide a battaglia in corso, col nemico sull'uscio, il fumo dei lacrimogeni che acceca e l'incendio che avvampa. In ogni caso nessun testimone mette in dubbio la sostanza del valore politico del gesto. A quasi 40 anni di distanza erravano quelli che pensavano che l'omicidio fosse perfetta allegoria del golpe e resta l'infamia di quelli che da destra e sinistra hanno irriso al gesto «vigliacco» del «piccolo borghese» cileno.

Siccome ogni tesi deve essere dimostrata, la miglior prova è il sacrificio della vita. Certo che avevamo paura che succedesse quello che poi è successo! Ma non era una paura... come la possiamo chiamare... una paura borghesuccia... No! Il presidente ha sacrificato la vita! Ci hanno offerto aerei, salvacondotti, il Chicho si sarebbe convertito in una grande figura internazionale... ma Salvador ha rifiutato tutto. Chi te lo leva dalla storia ora ${ }^{40}$ ?

\section{Conclusioni}

Dopo essere fuggito da Tomás Moro, Milton Silva si rifugia «da un compagno». Prendiamo lui tra le tante testimonianze del post-golpe. Pablo finisce allo Stadio nazionale, Renato vola subito in esilio, Manuel rivede la madre solo nel 1990, Jaime si nasconde tra le tombe del cimitero dove è nato. «La sera alla televisione vediamo il discorso della giunta sull'estirpare il marxismo. Mi sentivo malissimo, impotente. Il giorno dopo il padrone di casa ci chiede di andar via. Era pericoloso, è vero, ma sentii che ci stava cacciando». Dal «giorno pieno di radio» si passa a un discorso televisivo, dall'accoglienza alla paura. In poche ore è un altro Cile. Un altro Cile che incide così potentemente sull'immaginario dal far percepire come illecita qualunque rappresentazione di paese alternativa ${ }^{41}$.

\footnotetext{
40 Carlos Jorquera.

41 T. Moulian, Chile actual. Anatomía de un mito, Arcis, Santiago 1997.
} 
Il golpe - Manuel - cancellò tutte le conquiste e il neoliberismo con l'agroindustria esportatrice spazzò via i piccoli coltivatori. Oggi abbiamo solo braccianti che hanno perfino perso il desiderio del poter coltivare una terra propria. E così abbiamo una macroeconomia ricchissima e tre milioni di persone che non hanno da mangiare. Questo ci fa pensare che il nostro pensiero socialista resti vigente. Disgraziatamente il nostro partito finora ha solo continuato il progetto neoliberale lasciato dalla dittatura. Oggi i dirigenti stessi del nostro partito sono neoliberali.

Rivendicare la figura di Salvador Allende e allo stesso tempo difendere la privatizzazione dell'educazione o del rame e accettare l'impunità per le violazioni di diritti umani corrisponde alla discrasia che per il Cile attuale Tomás Moulian definisce «trasformismo» ${ }^{42}$. Per arrivarvi è necessario passare attraverso un processo di distillazione che include l'elusione di alcuni dei temi oggetto di questo saggio: la partecipazione in prima persona di Allende alla battaglia; l'uso dell'AK donato da Fidel Castro; la percezione generale dell'11 come una vera battaglia; i dettagli sui piani di difesa e sulla possibilità di uscita del presidente; la denuncia - particolarmente scomoda - dell'assenza o tradimento dei partiti; alcuni dettagli sulle circostante della morte e sulla comunicazione dell'eventuale decisione di resa. Per chi di quei fatti fu testimone e si considera leale alla memoria dei caduti (non sfugge qui il senso coercitivo che talvolta acquisisce tale lealtà), l'oblio diviene funzionale alla piena accettazione, da parte dello stesso partito di Allende, della correlazione di forze, della costituzione pinochetista, tuttora in vigore, del modello neoliberale e dell'impunità per le violazioni dei diritti umani.

Il Psch può rivendicare la figura di Allende come padre nobile o essere accusato di esserne traditore, a seconda di come e da chi l'11 viene raccontato. Per chi lo accompagnò, Allende per essere padre nobile dei governi della Concertazione deve essere tradito. Allende è padre nobile quando è rappresentato come agnello sacrificale di una dittatura che diviene brutalità astratta. Perciò ne vanno occultate le foto - rese pressoché introvabili - dove combatte sdraiato sparando da una finestra del secondo piano della Moneda ${ }^{43}$. Va celato il fatto che combatta con l'AK47 regalatogli da Fidel Castro (la foto di Allende che entra alla Moneda imbracciandolo invece è fin troppo nota), così come sparisce dall'intera storiografia cilena il fatto che nel 1976 il guerrigliero argentino Mario Roberto Santucho cada combattendo con la pistola semiautomatica Mauser regalatagli nel 1972 dal presidente cileno ${ }^{44}$. Sono dettagli che lo restituiscono al suo tempo storico rendendolo indisponibile all'uso pubblico odierno. Perciò i Gap sono scomodi. Sono parte della storia che il Partito socialista non ha più interesse a raccontare perché esplicita la

42 Ivi, pp. 31-80 e, sul trasformismo, 145-47. 89.

${ }^{43}$ Oltre che nelle testimonianze riportate in questo saggio cfr. Ó. Soto, El último día cit., p.

44 M. Seoane, Todo o nada. La historia secreta y la historia pública del jefe guerrillero Mario Roberto Santucho, Planeta, Buenos Aires 1991, pp. 173-74 e 285; mia intervista a Luís Mattini, ex segretario del Prt argentino. 
scelta di Allende che rifiuta la guerra civile ma non cede alla dittatura e non abiura il socialismo.

Quell'Allende è inconciliabile con José Miguel Insulza ${ }^{45}$, che nel 1998, da ministro degli Esteri, si oppone strenuamente al giudice Baltazar Garzón, opera verso il governo britannico contro l'incriminazione e l'arresto di Augusto Pinochet a Londra e non trova pace fino a quando il "paziente inglese" non viene riportato in patria, libero e impunito. Insulza, riportando Pinochet a casa, si comporta come ci si aspetta che la classe dirigente cilena si comporti. È l'irriducibilità di Allende, testimoniata l'11, a stridere con la riconciliazione basata sul dimenticare che, come sempre nella storia cilena ${ }^{46}$, restituisce il potere ai civili garantendo onori e impunità ai militari. Tra tutte le opzioni, guerra civile, esilio, assassinio, il suicidio è l'unica scelta irrimediabile che impedisce una riconciliazione che non passi per l'edulcorazione di Allende.

Lechner e Güell ${ }^{47}$ considerano infondata un'idea quasi cospirativa che informerebbe la transizione, l'impunità, la totale continuità dei governi della Concertazione col modello neoliberale imposto dalla dittatura. Eppure nessuna riconciliazione è possibile senza la totale discontinuità con l'allendismo. La battaglia della memoria intorno ad Allende conferma non una cospirazione, ma la giustezza dell'inquietudine dei superstiti nel vedersi restituito sugli altari un simulacro del presidente che conobbero.

Così Allende è padre nobile col discorso delle grandi alamedas, ma va tradito nel tentativo di stabilire una continuità tra il suo socialismo latinoamericanista, figlio della Seconda Internazionale, e quello riformato, fondomonetarista e interno al «Washington consensus» dei suoi successori, che non questionano l'impalcatura dello stato costruita da Augusto Pinochet e dai Chicago Boys di Milton Friedman e basata sull'autoritarismo ${ }^{48}$. La figura di Salvador Allende è troppo centrale nella storia del Cile democratico e troppo rilevante per quella dell'intera America per essere rinnegata. Ma può restare nell'album di famiglia del partito socialista, solo espungendone i tratti considerati demodé: il socialismo stesso, il latinoamericanismo, l'anti-imperialismo, la solidarietà alle guerriglie, la proprietà pubblica del rame, la giustizia sociale, declinati alla maniera di un dirigente politico che entra in parlamento per la prima volta durante la guerra civile spagnola. Le testimonianze dei superstiti della Moneda denudano tale contraddizione.

45 José Miguel Insulza, 1943. Militante del Mapu, consigliere del ministro degli Esteri di Allende, Clodomiro Almeyda. Confluito nel Psch, negli anni ' 90 fu figura di spicco della diplomazia cilena. Dal 2005 segretario generale dell'Organizzazione degli Stati americani.

${ }^{46}$ B. Loveman-E. Lira, Las suaves cenizas del olvido. Vía chilena de reconciliación política 1814-1932, Lom, Santiago 1999.

${ }^{47}$ N. Lechner-P. Güell, Construcción social de las memorias en la transición chilena, Ssrc, Montevideo 1998.

48 J.J. Brunner, La cultura autoritaria, Flacso, Santiago 1983. 


\title{
Né Est né Ovest: verso una nuova storia del '900 europeo?
}

\author{
Marco Bresciani
}

Neither East nor West: Towards a New History of Twentieth Century Europe? Karl Schlögel's collection of essays deals with some aspects of European twentiethcentury history concerning the past and present of multiethnic towns and areas, especially in Central-Eastern Europe. He focuses on the destruction of the urban civilization during the Iiww, on the forced movements of population in the ethnically mixed regions, on the place of Germany in the twentieth century and its connections with the East. The review discusses whether Schlögel's book conveys a new interpretation of contemporary European history, consistently beyond the East/West paradigm typical of the Cold War.

Key words: Eastern Europe, multiethnic towns, forced movements of population, European history 1914-1991

Parole chiave: Europa orientale, città multietniche, spostamenti forzati di popolazione, storia europea 1914-1991

In una celebre pagina dell'Apologia della storia, Marc Bloch racconta di aver accompagnato Henri Pirenne a Stoccolma:

Appena giunti, mi disse: "Che cosa andiamo a visitare come prima cosa? Sembra che vi sia un Municipio nuovissimo. Cominciamo di lì". Poi, come se volesse prevenire un mio moto di meraviglia, aggiunse: "Se fossi un antiquario, non avrei occhi che per le cose vecchie. Ma io sono uno storico. È per questo che amo la vita"l.

Da questo spirito pare animato il noto storico tedesco dell'Europa centrale e orientale, Karl Schlögel, il quale ha percorso in lungo e in largo lo spazio che era stato dominato dagli Imperi plurinazionali fino al 1917-'18, che fu l'epicentro delle catastrofiche «guerre civili europee» (1914-'45) e che è stato segnato dai regimi comunisti fino al 1989-'91. Raccolta di saggi in parte già pubblicati su riviste internazionali, in parte inediti, la sua ultima opera,

${ }^{1}$ M. Bloch, Apologia della storia, Einaudi, Torino 1998 (ed. or. Paris 1949), p. 36.

«Passato e presente», a. XXXI (2013), n. 88 
Arcipelago Europa. Viaggio nello spirito delle città, offre un florilegio di brillanti intuizioni e di più banali osservazioni, che rispecchia la mescolanza della meditazione storiografica con il reportage giornalistico. Questi saggi sono organizzati all'interno di quattro sezioni (Descrizione dei luoghi come diagnosi del tempo; Stratificazioni temporali dell'Europa; Percorsi di vita, spazi di pensiero; La lunga marcia verso est): loro filo conduttore è un complessivo approccio alla recente storia europea, che merita di essere discusso ${ }^{2}$.

Nell'opera precedente, Leggere il tempo nello spazio, Schlögel aveva già chiarito i suoi strumenti metodologici, proponendo una stimolante riflessione sul problema della complessità dei rapporti tra cronologia e geografia, tra tempo e spazio. Rifacendosi ad una varietà di fonti (dagli indirizzari alle cartografie, dalle mappe e dagli orarie delle ferrovie alle topografie delle città), spiegava come la riconfigurazione dello spazio storico e geopolitico dopo l'89 e il conseguente superamento della dicotomia tra Est e Ovest abbiano definito la necessità di un approccio di tipo nuovo - transnazionale ed europeo - ad una storia non più intesa come somma delle diverse (e per lo più contrapposte) narrazioni nazionali. In particolare, attraverso specifici sistemi di interconnessione tra rapporti spaziali e temporali, denominati «cronotopi», riteneva possibile cogliere «la sincronia dell' asincronico», ossia stratificazioni di esperienze e di memorie che trovano nelle città una particolare cristallizzazione ${ }^{3}$.

«Nel 1989 l'Europa è stata il palcoscenico dei "momenti storici" con i quali è finito il XX secolo e il teatro in cui sono entrati in scena gli attori della nuova Europa» (p. 1). Sviluppando questo approccio, le prime due sezioni di Arcipelago Europa si propongono di ripensare l'esperienza della «nuova Europa», delle sue molteplici identità e dei suoi stratificati retaggi: nella prima (Descrizione dei luoghi come diagnosi del tempo) lo storico muove dal presente al passato, nella seconda (Stratificazioni temporali dell'Europa) viceversa. Il punto di partenza di Schlögel è Marijampolé, cittadina lituana nei pressi del confine polacco, non lontana da quel che è considerato il centro geografico del continente europeo. Attraverso questo eccentrico, ma centralissimo osservatorio diventato luogo di scambio delle automobili di tutto il continente (da Est come da Ovest), lo "storico del presente" segue le traiettorie della «nuova Europa». Infatti, la mobilità e la circolazione sociale garantiscono - ben al di là di trattati ufficiali o dei dibattiti intellettuali - l'integrazione di fatto dell'Europa. «L'Europa, vittima nel XX secolo della smaniosa ricerca di una società etnicamente omogenea, torna a mescolarsi di nuovo» (p. 13). Se le ferrovie contribuirono in modo decisivo a costruire e collegare

2 K. Schlögel, Arcipelago Europa. Viaggio nello spirito delle città, Mondadori, Milano 2011 (ed. or. Marjampole: oder Europas Wiederkehr aus dem Geist der Städte, München 2005). Ringrazio Guido Franzinetti, Niccolò Pianciola e Antonio Ferrara per i loro commenti a questa recensione.

${ }^{3}$ Cfr. K. Schlögel, Leggere il tempo nello spazio. Saggi di storia e geopolitica, Mondadori, Milano 2009 (ed. or. München 2003). 
la società europea della Belle époque (e soprattutto l'Impero russo) - prima di favorirne la distruzione, facendosi strumento di mobilitazione di eserciti e deportazione di popoli -, camion, pullman e compagnie aeree low cost sono oggi i veicoli privilegiati di trasporto e comunicazione nella «nuova Europa». Paradossalmente, però, secondo lo storico tedesco, queste dinamiche unificatrici si rivelano soprattutto in forma frammentaria:

L'Europa della Guerra fredda non esiste più. Dov'erano spazi omogenei - "l'Est", "l'Ovest" - vi sono oggi frammenti, enclave, isole. Secondo alcuni è solo un'opera incompiuta, ma in realtà sono questi i tasselli della nuova Europa. Il rinnovamento assume la forma della disgregazione, almeno in questa fase (p. 57).

Questa «nuova Europa» si manifesta soprattutto nella «cultura polifonica e mista» delle città dell'Europa centrale e orientale, che è stata distrutta nel XX secolo, e che ora per frammenti pare riaffiorare (p. 40). L'interesse di Schlögel per la storia urbana, nutrito di competenze urbanistiche, architettoniche e sociologiche, non è nuovo nella sua vasta produzione: ad esempio, allo splendore artistico e culturale della San Pietroburgo di primo '900 e al terrore politico e sociale nella Mosca degli anni ' 30 ha dedicato due importanti volumi ${ }^{4}$. Nel suo ultimo libro le città multietniche sono concepite come spie o indizi di storie più ampie, e a lungo (spesso tuttora) fraintese o ignorate dalle singole storiografie nazionali. Queste città - che si formarono negli spazi imperiali plurinazionali (asburgico, tedesco, ottomano e russo) di cui riproducevano le stratificate pluralità sociali e nazionali - furono lacerate e spesso distrutte dalla nuova «Guerra dei trent'anni», nella prima metà del XX secolo. Alla loro storia la ricerca ha dedicato una particolare attenzione negli ultimi anni, sotto la sollecitazione degli attuali processi economici e sociali che hanno reso più porosi i confini nazionali ${ }^{5}$. Arcipelago Europa offre una campionatura di realtà urbane per lo più incontrate nel corso dei viaggi del suo autore e colte in un aspetto rivelatore del loro paesaggio, in un passaggio decisivo della loro storia, o in un rapporto peculiare con uno scrittore ad esse legato: per via d'induzione, tende poi a ricostruire problemi più generali, quali i rapporti tra le diverse comunità nazionali negli Imperi plurinazionali, $\mathrm{i}$ conflitti tra città e campagne, le città come laboratori della modernità. Molti dei saggi qui presentati sono esercizi di lettura del «testo» delle città: Pietro-

${ }^{4}$ K. Schlögel, Petersburg: Das Laboratorium der Moderne (1909-1921), Fischer, Frankfurt a. Main 2002; Id., Terror und Traum. Moskau 1937, C. Hanser Verlag, München 2008.

${ }^{5}$ Senza pretesa di completezza, cfr. N. Davies-R. Moorhouse, Microcosmo: l'Europa centrale nella storia di una città, Mondadori, Milano 2005 (ed. or. London 2002); M. Mazower, Salonicco, città di fantasmi. Cristiani, musulmani ed ebrei tra il 1430 e il 1950, Garzanti, Milano 2007 (ed. or. London 2004); C. Mick, Kriegsfahrungen in einer multietnischer Stadt: Lemberg 1914-1947, Harrasowitz, Wiesbaden 2010; C. King, Odessa. Genius and Death in a City of Dreams, WW. Norton, New York 2011; G. Thum, Uprooted. How Breslau became Wrochaw during the Century of Expulsions, Princeton UP, Princeton 2011 (ed. or. Berlin 2003). 
burgo, Czernowitz, Nižnij, Oradea, Brno, Bucarest, Berlino, Mosca, Košice, Budapest offrono esempi di una topografia urbana che aiuta a farci un'idea «di quanto ricca, e probabilmente felice, fosse l'Europa prima delle grandi catastrofi del Novecento» (p. 79).

I due saggi più suggestivi - e forse i più originali di tutto il libro - sono dedicati all' «urbicidio», in cui si intrecciano la storia delle città, l'interpretazione del '900 come epoca della violenza, la funzione di osservatorio privilegiato dell'Europa centrale e orientale. In L'uccisione delle città: l'Europa in guerra e Nuova urbanità nella nuova Europa Schlögel si focalizza sulla distruzione della civiltà urbana europea, che caratterizzò la seconda guerra mondiale, e sul meno violento, ma non meno deleterio processo di ricostruzione, che seguì nel lungo dopoguerra. Il problema del bombardamento delle città tedesche (come Colonia, Amburgo e Dresda) è dunque inserito all'interno di una prospettiva storica europea, particolarmente debitrice della sviluppo della riflessione di Hans Magnus Enzensberger ${ }^{6}$. Nella sua fenomenologia della distruzione della civiltà urbana - materiale oltreché metaforica -, Schlögel prende le mosse dalla necessità di comprendere «la portata della regressione antiurbana del XX secolo che si esprime nell'urbicidio» (p. 166). Quindi, conclude:

L'autodistruzione dell'Europa - e il ruolo particolare dei tedeschi in questa vicenda - si concretizza nella distruzione delle città. L'urbicidio non va visto semplicemente come danno collaterale dell'epoca delle guerre mondiali, ma come centrale punto d'attacco delle forze della violenza e della semplificazione contro quelle complesse formazioni di cultura, compromesso e coesistenza, che sono le città. La "rottura di civiltà" non avviene in astratto, ma in luoghi specifici e in un contesto preciso (p. 169).

Se è vero che l'urbicidio ha visto come primo e sperimentale teatro l'Europa occidentale (l'autore ricorda la distruzione aerea di Coventry o di Rotterdam, ma dimentica quella di Guernica, Madrid e Barcellona), sono però le traumatiche esperienze delle città dell'Europa centrale e orientale - rase al suolo come Varsavia, Breslau o Königsberg, annientate per fame come Leningrado, o svuotate e ripopolate come Wilno o Lwow - a fare del XX secolo «[u]n'epoca di immensa regressione, una serie di catastrofi per la cultura urbana, un secolo di urbicidi» (p. 182).

Posta in controluce rispetto all'apocalittico scenario di morte e distruzione del 1939-'45, la visione idilliaca e irenica delle città multietniche imperiali da parte di Schlögel non appare convincente: i rapporti di forza politici tra le opposte comunità nazionali, affermati in forme estreme nei territori imperiali e post-imperiali, sono diluiti dalle rappresentazioni di tolleranza e convivenza, coltivate da esigue élites sociali e culturali. Queste città furono invece at-

${ }^{6}$ H.M. Enzensberger, L'Europa in macerie. Uno scorcio, in Id., Zig Zag. Saggi sul tempo, il potere e lo stile, Einaudi, Torino 1999, pp. 29-48 (ed. or. Frankfurt am Main 1990). 
traversate da tensioni crescenti tra le diverse comunità nazionali (e religiose), esasperate poi da quella dinamica di guerra e rivoluzione che condusse al crollo degli Imperi plurinazionali tra il 1917 e il 1918. Un indizio di questa lettura si trova nella tendenza ad associare la diversa toponomastica nazionale in una sola sequenza "multiculturalista" (ad esempio, Lemberg/Lwów/ L'vov/L'viv oppure Vilna/Wilno/Vilnius), la quale, trascurando il fatto che di volta in volta fu solo una di queste denominazioni a prevalere, comporta un'attenuazione, se non un vero e proprio fraintendimento, delle aspre competizioni politiche, culturali e sociali tra le diverse identità nazionali. D'altro canto, rispetto a un paradigma interpretativo rigidamente basato sulla svolta del 1989, le successive guerre nei Balcani e nel Caucaso che hanno insanguinato gli anni '90 (e oltre) aprono una contraddizione che Schlögel elude. Non può infatti non meravigliare, nella sua riflessione sull'urbicidio, l'assenza di Vukovar, Sarajevo, Srebrenica, Groznij. Le guerre nella ex-Jugoslavia, come quella in Cecenia, sono menzionate solo nella misura in cui hanno imposto all'agenda storiografica il problema della «pulizia etnica», spingendo a studiare le migrazioni forzate e gli spostamenti di popolazione che hanno caratterizzato la storia europea recente. Al contrario, una più analitica attenzione per i conflitti nazionali nel contesto di disfacimento delle due federazioni comuniste (l'Unione Sovietica e la Jugoslavia) avrebbe consentito di riconoscere, oltre le rotture, i molteplici e sottili fili di continuità che si dipanano intorno al 1989 e che segnano la storia di longue durée dei processi di costruzione statale e nazionale soprattutto (ma non solo) nelle regioni ex-imperiali dell'Europa centrale e orientale ${ }^{7}$.

Alcuni dei temi fin qui trattati sono ripresi in una diversa prospettiva nella terza sezione del libro (Percorsi di vita, spazi di pensiero), dove dall'incrocio di biografie e opere di quattro personaggi che tra loro hanno poco o nulla in comune (Harry Kessler, Erwin Dwinger, Sándor Márai, György Lukács) emerge una nuova topografia storica, caratterizzata dalle «zone di scontro della guerra civile europea» (p. 187).

Infine, nella quarta sezione (La lunga marcia verso est) Schlögel affronta il tema della collocazione della Germania dopo il 1989, nel quadro della storia europea. Il suo principale obiettivo polemico è la storia della Germania di Heinrich August Winkler, basata sull'idea di «un lungo cammino verso Occidente» ${ }^{8}$. Secondo Schlögel, invece, «il futuro della Germania è legato, come in passato, agli Stati e ai popoli dell'Europa orientale» (p. 243): proprio perché il 1989 ha svincolato la Germania dall'“Occidente", è diventato possibile fare i conti con tutti gli aspetti - anche quelli più complicati e dolorosi - del suo passato quale la fuga delle popolazioni tedesche dall'Europa centro-

\footnotetext{
7 È significativo, ad es., che Schlögel non menzioni l'importante riflessione sull'urbicidio da parte dell'architetto serbo, già sindaco di Belgrado, Bogdan Bogdanoviç nel contesto delle guerre jugoslave degli anni ' 90.

${ }^{8}$ H.A. Winkler, Grande storia della Germania. Un lungo cammino verso Occidente, 2 voll., Donzelli, Roma 2004 (ed. or. München 2000).
} 
orientale sotto l'avanzata dell'Armata rossa e la loro espulsione dalla Prussia orientale, dalla Polonia occidentale, dalla Boemia, dalla Romania, dalla Slovenia e dalla Vojvodina nell'immediato dopoguerra. Attraverso questo ampliamento della prospettiva geografica, Schlögel ricolloca il problema della «perdita dell'Est tedesco» (p. 249), prendendo una maggior distanza storica dal «passato che non passa» e al contempo tentando un recupero critico e selettivo dell' Historikerstreit (1986-'87) - il famoso dibattito pubblico e storiografico in cui interpretò un ruolo di primo piano, oltre ad Ernst Nolte, Andreas Hillgruber ${ }^{9}$. La tesi più provocatoria di Hillgruber - tesa ad assumere il punto di vista dei tedeschi del 1944-'45 e ad associare il tramonto dell'ebraismo europeo a quello delle comunità tedesche in Europa orientale - è riproposta in un diverso contesto storiografico. "Quest'opera di distruzione dell'Europa, caratterizzata da una violenza senza precedenti e culminata nell'Olocausto, ebbe come esito il crollo totale della posizione tedesca nell'Europa centro-orientale» (p. 259). A differenza di Hillgruber, Schlögel inserisce lo sterminio degli ebrei di Europa, pur associato alla deportazione e allo sradicamento delle comunità tedesche dell'Europa centro-orientale, all'interno di un più lungo ciclo storico cominciato con la Grande Guerra, con la disgregazione delle costruzioni imperiali plurinazionali e la conseguente competizione per la formazione di nuovi Stati nazionali, culminato nella seconda guerra mondiale, ma protrattosi fino alla fine dell'Unione Sovietica e della Jugoslavia. Forse però, sarebbe stato opportuno precisare che la crisi di quelle comunità tedesche centro-orientali era stata avviata dalle politiche di persecuzione degli ebrei tedeschi (politicamente e culturalmente assimilati), che di quelle comunità erano una componente essenziale (diverso fu il caso degli Ostjuden).

Partendo dallo specifico nesso tra storia tedesca ed Est Europa, Schlögel si misura con il problema più generale delle migrazioni forzate, della «pulizia etnica», della «rivoluzione sociodemografica» che sconvolse la topografia sociale e nazionale dell'Europa durante e dopo la seconda guerra mondiale. Da questo punto di vista, l'Est Europa è stato «una sorta di immensa stazione di scambio» (p. 246). Pur ricordando l'importanza di opere pionieristiche come Europe on the Move di Eugene M. Kulischer (1948) e Population Transfers in Europe di Joseph B. Schechtman (due volumi, riguardanti il 1939-'45 e il 1945-'55, rispettivamente datati 1946 e 1962), lo storico tedesco pone l'accento sugli studi che, sull'onda delle guerre balcaniche e caucasiche degli anni '90, si sono concentrati in forma analitica e sistematica su deportazioni e spostamenti di popolazione. In questo modo, trascura una significativa messe di opere che avevano collocato queste dinamiche all'interno di problemi più generali, quali la definizione dei confini e la formazione dei regimi post-bel-

9 A. Hillgruber, Il duplice tramonto. La frantumazione del "Reich" tedesco e la fine dell'ebraismo europeo, il Mulino, Bologna 1990 (ed. or. Berlin 1986); Id., La distruzione dell'Europa. La Germania e l'epoca delle guerre mondiali (1914-1945), il Mulino, Bologna 1992 (ed. or. Berlin 1988). 
lici. D'altro canto, sottovaluta gli effetti distorcenti che l'insistenza sulla «pulizia etnica» può comportare a livello interpretativo, riducendo alla dimensione "etnica" quel grumo spesso indissolubile di fattori politici, sociali e nazionali che hanno modellato tutta l'esperienza della storia europea e che hanno segnato in forma particolarmente acuta i processi di costruzione statale nelle aree plurinazionali, fin dalla lunga vigilia della prima guerra mondiale ${ }^{10}$.

In numerosi passaggi di Arcipelago Europa, il presente si oppone al passato recente, e si ricollega al passato più remoto. Così, in contraddizione con la lezione di Bloch prima evocata, il presente non solo suggerisce le domande, ma detta anche le risposte allo storico, spingendolo a un atteggiamento anacronistico verso il passato. È una delle possibili, e forse la più grave, delle deformazioni prospettiche a cui può condurre quella «storia del tempo presente» che è stata teorizzata da un altro noto viaggiatore dell'Europa centrale, Timothy Garton Ash. Non a caso, la stessa riflessione di Schlögel può essere considerata un frutto maturo del dibattito (più politico che scientifico) intorno alla Mitteleuropa, che si sviluppò tra la fine degli anni '70 e la metà degli anni ' 80 e che non di rado gravitò intorno ad una nostalgica evocazione del «mondo di ieri» (ispirata da Stefan Zweig) ${ }^{11}$.

Il '900 di Schlögel, che si chiude con il 1989, si apre con il 1914, con quella Grande Guerra definita come la «Urkatastrophe europea» del XX secolo (p. 79). Se questa periodizzazione è stata canonizzata dal Secolo breve di Eric Hobsbawm, ben diverse appaiono però le implicazioni che ne trae lo storico tedesco. Tra i due termini cronologici, il 1989 e il 1914 - evocati implicitamente da frequenti espressioni come «ritorno al punto di partenza», «ritorno alla normalità», o «ritorno alla complessità» - si viene infatti a creare un corto circuito. Il periodo precedente il 1914 e quello successivo al 1989 sembrano privi di quelle contraddizioni e di quei conflitti che invece segnarono - in forme estreme - l'arco centrale del secolo. Da un lato, non si presenta alcun tentativo di connettere all'interno di cicli storici più lunghi il crollo degli Imperi nel 1917-'18 con le rivoluzioni del 1848 in Europa centrale, o con quella del 1905 nell'Impero russo. Dall'altro, non si compie alcuno sforzo di comparare il riassetto europeo successivo alla Grande Guerra con le trasformazioni geopolitiche del 1989-'91 e il loro lascito di conflitti e instabilità. In questo modo, il '900 finisce per apparire come una parentesi, entro cui si è svolta «sostanzialmente una storia di violenza» (p. 257). Da questo punto di vista, Schlögel sviluppa in modo coerente un approccio in parte già

${ }^{10}$ Cfr. H.H. Hahn-E. Hahnova, Alte Legenden und neue Besuche des "Ostens". Über Norman M. Naimarks Geschchtsbilder, «Zeitschrift fūr Geschchtswissenschaft», 54 (2006), n. 7-8, pp. 687-700, recensione a N. Naimark, La politica dell'odio. La pulizia etnica nell'Europa contemporanea, Laterza, Roma-Bari 2002 (ed. or. Cambridge 2001). Cenni critici in questo senso anche in A. Ferrara-N. Pianciola, L'età delle migrazioni forzate. Esodi e deportazioni in Europa 1853-1953, il Mulino, Bologna 2012, pp. 13-38.

${ }^{11}$ Cfr. G. Franzinetti, Mitteleuropa in East-Central Europe from Helsinki to EU Accession (1975-2004), «European Journal of Social Thought», 11 (2008), pp. 219-35. 
adottato da Mark Mazower. Tuttavia, a differenza de Le ombre dell'Europa, Arcipelago Europa, che tende ad identificare il vecchio continente con le esperienze traumatiche delle sue regioni centrali e orientali, soffre di quella distorsione retrospettiva che isola il problema della violenza dalle culture e dalle prospettive politiche che l'hanno giustificata ed alimentata. Questa narrazione complessiva del ' 900 s'inscrive in quell'orizzonte interpretativo ampiamente diffuso nell'ultimo trentennio - che assume prevalentemente il punto di vista delle vittime ${ }^{12}$.

Ripensando la tradizione geopolitica tedesca (soprattutto Friedrich Ratzel e Karl Lamprecht) alla luce dell' ispirazione di Walter Benjamin, di Carl Schorske e di Reinhardt Koselleck, Schlögel ha sviluppato un innovativo approccio storico «topograficamente sensibile», che ha imposto la necessità di coordinare consapevolmente il tempo con lo spazio ${ }^{13}$. E un approccio che ha trovato sponde diverse nella storiografia più recente. Timothy Snyder ha fatto dello spazio compreso tra il Baltico e il Mar Nero (le «terre di sangue») l'osservatorio privilegiato per comprendere le dinamiche totalitarie, le interazioni e i conflitti tra Hitler e Stalin ${ }^{14}$. Più in generale, Dan Diner e Tony Judt invece hanno scelto una città dell'Europa centrale o orientale come punto d'osservazione per inquadrare le vicende contemporanee del vecchio continente, troppo spesso identificato con la sua metà "occidentale". Per raccontare il '900 Diner si è immaginato sulla famosa scalinata del porto d'Odessa (immortalata da Sergej Eizenštein), spettatore delle tragiche convulsioni dei territori multietnici imperiali dell'Europa centro-orientale, a partire dalla rivoluzione russa del 1905. Judt si è raccontato viaggiatore sulle pensiline della Westbahnhof di Vienna nell'autunno del 1989, per ripensare, a ritroso, gli incroci densi di storia tra le diverse vie dell'Europa centrale e mettere così in discussione il tradizionale confine tra Est e Ovest nel dopoguerra ${ }^{15}$.

Schlögel ritiene che solo con il superamento dell' «elemento binario e dicotomico» della logica inerente alla guerra fredda, si sono create le condizioni per la «formazione di un nuovo quadro di rifermento, di un nuovo spazio discorsivo paneuropeo, transfrontaliero e transnazionale» (p. 251). Arcipelago

12 Cfr. T. Judt, L'età dell'oblio, Laterza, Roma-Bari 2009 (ed. or. New York 2008), pp. 325; E. Traverso, Il secolo armato. Interpretare le violenze del Novecento, Feltrinelli, Milano 2012, pp. 168-195. Il riferimento è ovviamente a M. Mazower, Le ombre dell'Europa. Democrazie e totalitarismi nel XX secolo, Garzanti, Milano 2000 (ed. or. London 1998).

${ }^{13}$ K. Schlögel, History "takes place". Problems of a Topographically Sensitive Historiography, paper presentato al seminario "Why People Like to Live in Cities" (The Hague, 10 novembre 2009), www.studentencorps.com/urbansciencesdenhaag/Karl\%20Schlogel\%20 Den\%20Haag\%202009.pdf.

${ }^{14}$ Cfr. T. Snyder, Terre di sangue. L'Europa nella morsa di Hitler e Stalin, Rizzoli, Milano 2011 (ed. or. New York 2010).

15 Cfr. D. Diner, Raccontare il Novecento: una storia politica, Garzanti, Milano 2001, pp. 11-14 (ed. or. München 1999) e T. Judt, Dopoguerra. Una storia d'Europa dal 1945 a oggi, Mondadori; Milano 2007, pp. 3-4 (ed. or. London 2005). Al suo approccio "topografico" alla storia del dopoguerra europeo Judt fa cenno in The Memory Chalet, The Penguin Press, New York 2010, pp. $70-71$ (trad. it. Lo chalet della memoria, Laterza, Roma-Bari 2011). 
Europa offre dunque un efficace antidoto alla tentazione del Sonderweg (tedesco ma non solo): sulla necessità di una storia europea, refrattaria e irriducibile agli imperativi e ai confini delle storiografie nazionali, converge con la riflessione di Norman Davies ${ }^{16}$. Tuttavia, pur cercando di sfuggire alla logica dicotomica della guerra fredda, lo stesso Schlögel però finisce per privilegiare le esperienze dell'Europa centrale e orientale. Se la guerra fredda aveva portato a identificare indebitamente l'Europa occidentale con l'Europa tout court, il post-guerra fredda ha indotto a concentrarsi oltremisura sul punto di vista dell'Europa orientale per la comprensione del secolo scorso ${ }^{17}$. Sarebbe forse necessario accantonare definitivamente l'idea di una prospettiva geografica "privilegiata" (che siano "l'Est" o "l'Ovest", variamente intesi) e ripensare i problemi della storia contemporanea entro una prospettiva europea comparata, insieme policentrica e unitaria, capace di ricostruire interazioni e conflitti su diversi livelli (locali, nazionali e continentali) e di riconoscerne i rapporti con le aree extraeuropee ${ }^{18}$. Tuttavia, la lettura di Arcipelago Europa conferma che la guerra fredda, che sul piano geopolitico e ideologico si è chiusa tra l' 89 e il ' 91 , ha lasciato un'eredità intellettuale duratura, che arriva fino ai giorni nostri: il muro di Berlino continua ad attraversare le menti di non pochi europei, proiettando la sua ombra ben al di là della sua caduta.

${ }^{16}$ N. Davies, Europe East\&West, Pimlico, London 2007. Nel suo ultimo libro, Davies introduce gli «Stati esistenti» all'interno di una lunghissima storia europea di «Stati estinti» (Vanished Kingdoms. The Rise and Fall of States and Nations, Allen Lane, London 2011).

17 Per la persistente accezione di «Europa orientale» nel post-guerra fredda e per i suoi usi anacronistici cfr. M. Confino, Reinventing the Enlightenment: Western Images of Eastern Realities in the Eighteenth Century, «Canadian Slavonic Papers/Revue Canadienne des slavistes», 36 (1994), n. 3-4, pp. 505-22 e G. Franzinetti, The Idea and the Reality of Eastern Europe, «History of European Ideas», 34 (2006), n. 4, pp. 361-68: entrambi discutono criticamente L. Wolff, Inventing Eastern Europe: The Map of Civilization on the Mind of the Enlightenment, Stanford UP, Stanford 1994.

${ }_{18}$ Cfr. T. Judt (with T. Snyder), Thinking the Twentieth Century, W. Heinemann, London 2012, pp. 282-83. 
Copyright $(\odot$ FrancoAngeli

N.B: Copia ad uso personale. È vietata la riproduzione (totale o parziale) dell'opera con qualsiasi mezzo effettuata e la sua messa a disposizione di terzi, sia in forma gratuita sia a pagamento. 


\title{
Fumetti nella storia, storia nei fumetti
}

\author{
a cura di Roberto Bianchi
}

\section{Michel Porret (sous la direction de) \\ Objectif bulles. \\ Bande dessinée \& histoire \\ Georg, Genève 2009, pp. 302, ill.}

In uno dei pochi saggi disponibili sulla storia della storiografia del fumetto in Italia - pubblicato in occasione del centenario del «Corriere dei Piccoli», la cui nascita continua a essere considerata, da molti, il termine a quo per la comparsa dei fumetti nella penisola, con una periodizzazione rigida e oramai obsoleta - , si sottolinea l'assenza di un'aggiornata analisi delle caratteristiche, delle peculiarità, delle trasformazioni e delle ricadute sociali del fumetto nell'Italia contemporanea: una storia ancora «tutta da scrivere» (Fabio Gadducci-Matteo Stefanelli, La storiografia del fumetto in Italia. Tradizioni e strategie culturali, in Il secolo del fumetto. Lo spettacolo a strisce nella società italiana 1908-2008, a cura di Sergio Brancato, Tunué, Latina 2008, p. 130).

Se è relativamente più ampia la letteratura sui periodici per l'infanzia e la gioventù che pubblicavano anche fumetti, sulle biografie di singoli autori e personaggi, come sulle genealogie di scuole e stili artistici, o sul ruolo pedagogico, sui linguaggi e sulla lingua della Nona arte (definizione resa celebre da Francis Lacassine, Pour un 9e art. La bande dessinée, Union général d'éditions, Paris
1971), in Italia mancano tuttora riconosciuti e consolidati punti di riferimento storiografici sull'uso del fumetto nell'ambito della ricerca scientifica, magari persino capaci di connettere metodi e strumenti di analisi delle varie discipline al fine di usare meglio le potenzialità di una fonte preziosa per lo studio di società, cultura, processi di alfabetizzazione, forme di propaganda, linguaggi del mondo contemporaneo. Anche per questo, si ritiene utile passare in rassegna una serie di testi recenti che - con approcci, chiavi di lettura e risultati diversi - esaminano o le forme di rappresentazione del passato nei fumetti, oppure la storia e le origini dei fumetti e del loro mercato editoriale.

È vero che negli ultimissimi anni si è iniziata ad abbozzare una nuova storiografia del fumetto anche in Italia e che da alcune esperienze emergono segnali importanti; penso alla nuova collana "Nerbiniana", attiva da un anno, volta peraltro a definire e distinguere il genere fumetto all'interno di una produzione di «stampa periodica per l'infanzia e la gioventù» relativamente vasta; $o$ alla rivista «Signs-Studies in Graphic Narratives», pubblicata a Pisa con un'attenzione peculiare all'importanza dei supporti e delle tecniche di produzione dei fumetti, per giovani e adulti, nel corso del tempo; o a qualche attività avviata da tempo, anche sul piano didattico, in alcuni atenei ita- 
liani. Eppure è ancora utile gettare uno sguardo oltre i confini nazionali per incontrare percorsi di indagine e riflessioni che fino a oggi hanno trovato poca eco nel nostro paese, a dispetto dei numerosi e appassionati dibattiti che animano tanti blog, siti internet, riviste, fiere e incontri organizzati in decine di province italiane da chi del fumetto fa il proprio mestiere, senza però infrangere la distanza col mondo della ricerca storica e dell'università, salvo poche e lodevoli eccezioni.

Il volume curato da Michel Porret ordinario di storia moderna all'Università di Ginevra - rappresenta sicuramente un buon punto di riferimento per lo studio della storia culturale del fumetto (bande dessinée: $B D$ ) dalla fine dell' 800 ai giorni nostri, un prodotto letterario che (nella sua versione moderna, meglio precisare) è effettivamente esploso col XX secolo, così come il cinema, pur avendo una lunga storia alle spalle - che però in quest'opera non è presa in considerazione. Il curatore adotta, infatti, una periodizzazione determinata dal legame, ritenuto discriminante con la produzione precedente, tra fumetti e diffusione di massa della stampa periodica.

A lungo svalorizzata da censori e moralisti, sempre pronti a opporre la $B D$ alla "grande letteratura", l'ascesa del fumetto si intreccia indissolubilmente con quella della stampa di età industriale e dei periodici per la gioventù e l'infanzia, almeno fino agli anni '70 del '900. Sulla scia dei romanzi popolari d'avventura dell'800 spiega Porret in una solida e ben leggibile introduzione dal titolo intrigante: La bande dessinée éprouve l'historie (pp. 11-41) -, la $B D$ declina in modo originale una «mistica moderna» legata alla figura romantica e individualista dell'avventuriero nelle sue diverse vesti: militare, scienziato, esploratore, marinaio, globetrotter, sportivo, detective o giornalista d'inchiesta, comunque quasi sempre difensore dei deboli e raddrizzatore dei torti.

Prodotto culturale di consumo di massa, se il fumetto contemporaneo è espres- sione di un'arte e di un artigianato sorti in ambienti urbani in coincidenza con l'industrializzazione della cultura, l'emergere della stampa popolare e le grandi tirature dei giornali nella belle époque (p. 13), oggi quello del fumetto mondiale rappresenta un mercato dalle dimensioni colossali, con cifre d'affari che, se appaiono importanti per i paesi francofoni dove nel 2003 gli editori Dupuis, Dargaud e Glénat dichiaravano tra i 50 e i 75 milioni di euro per la sola $B D-$, sono maggiori per il mercato nordamericano (pur in flessione da qualche tempo) e decisamente notevoli per quello asiatico: $\mathrm{mi}$ risulta che, sempre nel 2003, gli editori giapponesi Kodansha e Shogakukan sfioravano vette di 1.500 e 1.400 milioni di euro grazie ai loro manga. Il caso italiano è, ora, ben diverso in termini di cifre d'affari, per quanto da sempre animato da autori, promotori e editori dai forti legami internazionali; ma il volume di Porret centra l'attenzione su esperienze di altri paesi.

Per immergersi nella vasta prateria della $B D$, il curatore ha raccolto undici contributi di autori francofoni (otto svizzeri, equamente ripartiti tra le Università di Ginevra e Losanna, e tre francesi) che concorrono a ripensare la storia culturale del fumetto, offrendo approcci e punti di vista anche variegati. Sono ricerche che spaziano dalle storie «d'infanzia» di un secolo fa del losannese Alexandre Steinlen (1859-1923), esaminate da Philippe Kaenel, alla lunga fila di fumetti dedicati al Gesù storico, riletti da Alain Boillat. Dalla debordante presenza di medioevo nei fumetti, analizzata da Alain Corbellari (già autore di un'ampia e ormai superata bibliografia sul tema («Études de Lettres», 1, 2001), alle rappresentazioni del carcere nelle $B D$ tra le due guerre mondiali, interpretate da Frédéric Chauvaud. Dalle tensioni tra "Spagna ufficiosa" e "Spagna ufficiale" nei tebeo durante il franchismo (Viviane Alary), alla Shoah nei fumetti (Yan Schubert). Dalle rappresentazioni del collaborazionismo 
nelle avventure di Asterix (Sébastien Farré) a quelle della guerra nucleare nei fumetti francesi post 1945 (M. Porret); fino allo specifico caso dei fumetti di Tintin, letti per linee interne (le rappresentazioni delle rivoluzioni latinoamericane nell'opera di Hergé, esaminate da Philippe Marguerat) o per le ricadute nell'ambito del collezionismo e della produzione editoriale (Olivier Roche), per chiudere con l'analisi di un'opera del 1991 di Alan Moore (From Hell, studiato da Danielle Chaperon).

Dall'insieme dei saggi, spesso corredati di illustrazioni a colori, emerge una forte attenzione a interpretare l'immaginario del fumetto come specchio (sappiamo che F. Lacassin parlava della $B D$ come miroir du monde, allo stesso modo di ogni altra produzione intellettuale) di quel contesto socioculturale di produzione e diffusione dei fumetti che ne condiziona le figure, gli archetipi, i codici narrativi, le rappresentazioni raffigurate, piuttosto che a leggere il fumetto come forma d'arte dotata di una peculiare storia della sua grammatica e dei suoi temi interni. Quasi a riaprire implicitamente una discussione cara a Ernst Gombrich mezzo secolo fa - però centrata, all'epoca, sulle arti figurative -, l'équipe guidata da Porret mostra i legami imprescindibili tra l'immaginario della $B D$ e l'ambiente di produzione e diffusione di albi e periodici. Il contesto sociopolitico «formatta la storicità culturale» della $B D$ (pp. 21-22). I fumetti, insomma, sono figli del loro tempo.

Eppure, va aggiunto che pur trattandosi di un prodotto che deve vendere, figlio della società di massa e di un mercato dominato da colossi dell'editoria, il fumetto contemporaneo resta un'arte povera che a prezzi relativamente contenuti può rappresentare con immagini, margini e parole, scenari e mondi infiniti, con una libertà diversa rispetto a quella concessa - per esempio - a cinema o televisione, e con una grammatica interna, propria, dotata di una storia peculiare, non sempre strettamente determinata dal contesto di produzione e dalle imposizioni mercantili. Lo stile dei fumetti, insomma, è un indicatore problematico dello "spirito di un'epoca". Un rilevatore importante, ma sempre da interpretare; pena il rischio di ridurlo a espressione automatica di trasformazioni sociali, culturali, economiche.

L'apparato bibliografico che correda ogni saggio (prevalentemente basato su testi francofoni e anglosassoni) arricchisce un'importante opera collettanea che, se non esaurisce il tema, come dichiarano gli autori, ha anche il pregio di mostrare e analizzare la Storia come oggetto dei fumetti e come solo uno dei volti della storia dei fumetti, evocando le rappresentazioni del mondo come inesauribile fonte d'ispirazione realistica e al contempo drammatica per l'immaginario sociale della $B D$.

Roberto Bianchi

\section{Patrick Gaumer}

Dictionnaire mondial de la BD

Larousse, Paris 2010, pp. 954+96, ill.

Frutto di un lavoro imponente, della stratificazione, del riesame e dell'arricchimento di voci e richiami già comparsi nelle edizioni precedenti - 1994, 1998 e 2004, le prime redatte da Patrick Gaumer (ex libraio, ora saggista e docente universitario) col compianto Claude Moliterni (fumettista, figura centrale del $\mathrm{co}$ micdom internazionale e, tra l'altro, cofondatore dei festival di Lucca e Angoulême), con le sue 2.200 voci e 1.200 illustrazioni il dizionario si presenta come un'enciclopedia che intende offrire un rinnovato strumento di informazione critica globale sui diversi aspetti della storia e della produzione odierna dei fumetti.

Come si legge nella breve premessa, o negli interventi e nelle interviste rilasciate da Gaumer (in parte rintracciabili su internet), l'opera non si dichiara esaustiva e non discende solo da intenti divul- 
gativi, ma da considerazioni di tipo culturale e, per certi aspetti, di tipo storiografico. In particolare, questa nuova edizione del dizionario differisce dalle precedenti, oltre che per alcune correzioni, per tre aspetti: la maggior attenzione verso i "pionieri" del fumetto moderno come verso i protagonisti delle più recenti produzioni "indipendenti"; il grande spazio concesso ai manga (probabilmente sollecitato anche dall'invasione dei fumetti giapponesi nell'Esagono, registrata negli ultimi vent'anni: la quindicina di titoli tradotti nel 1993, adesso va moltiplicata per cento); il tentativo di offrire una cornice effettivamente completa dell'attuale mondialisation della produzione di $B D$.

In realtà, le assenze e le lacune interne al quadro disegnato dai testi che compongono il dizionario - ovviamente numerose - forse non sono sempre condivisibili; d'altronde, una vera e propria enciclopedia completa ed esaustiva dei fumetti mondiali resta ancora da scrivere. Eppure questo dizionario è una miniera di informazioni gradevole da leggere e facile da consultare, grazie anche all'indice analitico collocato alla fine del volume. Autori e autrici, promotori e organizzatori culturali, albi e personaggi, termini tecnici, club e associazioni, periodici e serie sono presentati in ordine alfabetico, con numerosi richiami interni tra una voce e l'altra.

Particolarmente interessante è l'inserto a colori Chic Planète, composto di un centinaio di pagine e collocato al centro del volume, dove in ordine alfabetico sono suddivise le aree geografiche, e in special modo linguistiche, di presenza e diffusione dei fumetti nel mondo. Ognuno dei 25 capitoli è articolato in vari paragrafi dedicati a specifici gruppi di paesi della relativa area, o a momenti, fasi, tendenze e protagonisti che hanno segnato la storia del fumetto in una determinata zona: Africa, Germania, Argentina, Australia e Nuova Zelanda, Belgio francofono, Brasile, Cina, Corea del Sud,
Danimarca, Spagna, Stati uniti d'America, Finlandia, Fiandre e Paesi Bassi, Francia, Gran Bretagna, Islanda, Italia, Giappone, Messico, Norvegia, Portogallo, Québec, Svezia, Svizzera tedesca, Svizzera romanda.

Lo spazio esaminato è dunque molto ampio, con aperture orizzontali che hanno l'ambizione di offrire un quadro globale, e schemi discorsivi verticali che sintetizzano e suddividono la storia del fumetto per aree linguistiche, nazionali, territoriali. Si tratta quindi di uno strumento assi utile, che traccia le coordinate generali per orientarsi tra bande dessinée, strip cartoon o comic, manga, manhua e manhwa, banda desenhada o quadrinho, historieta o tebeo, fumetti, romanzi a fumetti e graphic novel e che rispecchia il progressivo, crescente métissage culturel che ha segnato il mondo del fumetto nell'ultimo secolo e che, per certi versi, potrebbe tendere a istituire un canone del fumetto mondiale.

Nonostante la ricchezza dell'opera, e le fatiche per realizzarla, il prodotto risente inevitabilmente di uno sguardo che dal dinamico punto zero di Parigi guarda il mondo circostante. Ad esempio, le 10 pagine dell'importante e innovativo capitolo su l'Afrique - scritto a quattro mani assieme a Christophe Cassiau-Haurie, già autore di saggi sulla $B D$ in Africa e nell'Oceano Indiano - riguarda quasi esclusivamente l'area francofona del continente, dove, scrivono gli autori, la storia della $B D$ contemporanea realizzata da non europei si aprì nel 1958 sulle pagine di «Antilope», rivista del Congo belga. La produzione africana è letta per grandi aggregati. Si guarda quindi alle vicende dell'Afrique centrale (Repubblica democratica del Congo, Repubblica del Congo, Ciad, Repubblica Centrafricana, Repubblica Equatoriale, Burundi, Camerun, Ruanda, Gabon) e dell'Afrique de l'ouest (con i pochi autori conosciuti del Sahel e un'attenzione più rivolta alle recenti produzioni in Benin, Burkina $\mathrm{Fa}-$ so, Mali, Senegal - patria di Goorgoor- 
lou, uno dei personaggi più famosi del continente -, e Costa d'Avorio, dove spiccano le avventure di Aya di Yopougon, tradotte anche in italiano, fino ad arrivare ai fumetti di Gibuti e a quelli del Madagascar, il cui punto di svolta fu rappresentato dalla prima $B D$ malgascia, $N y$ Ombalahibesamo, pubblicata all'indomani dell'indipendenza e dedicata ad Andrianampoinimerina). Quindi si risale al Magreb, con le diverse vicende dei fumetti in Marocco (anche in lingua berbera), Algeria (segnate dallo stillicidio di autori, uccisi o scomparsi, e dalla chiusura di molti periodici negli anni '90) e Tunisia. Solo poche righe finali sono riservate alla produzione africana anglofona, facendo riferimento alla presenza della rivista sudafricana «BitterKomix» al Festival di Angoulême, per un grande e variegato continente i cui fumetti si diffondono più grazie a internet che alla carta stampata.

Colpiscono, inoltre, alcune disomogeneità nei criteri adottati per mostrare le peculiarità storiche della $B D$ nelle varie aree esaminate. Ad esempio, per descrivere il complesso mondo del fumetto cinese si parte dai nianhua, e in particolare dalle stampe a colori d'epoca Ming e Qing, per poi rievocare la popolarizzazione del termine manhua tra le due guerre mondiali e giungere alle tendenze attuali, distinguendo puntualmente, in paragrafi separati, fra la tradizione fumettistica dominante nella Repubblica popolare e le originalità di Hong Kong. Invece, per ricostruire le vicende del fumetto italiano è proposta una storia di breve periodo, tutta novecentesca, la cui genesi è individuata nel dicembre 1908 , col primo numero del solito «Corriere di Piccoli». Sta di fatto che anche molti autori e prodotti italiani sono citati nell'inserto a colori e nell'insieme del dizionario, come il vercellese Angelo Agostini, considerato da molti il "padre fondatore" del moderno fumetto brasiliano negli anni '60 dell' 800 , o come l'irrequieto e geniale Andrea Pazienza. Per entrambi, e per altri, forse sarebbe stato utile riservare specifiche voci biografiche, come quella dedicata a Gian Luigi Bonelli, cui non fa però seguito un articolo per il figlio Sergio Bonelli (comunque citato sette volte nel volume).

Ma piuttosto che dedicarsi a una caccia alle assenze o agli squilibri nazionali, in un'opera generosa e costruita con intelligenza, giova usare le oltre mille pagine del volume per conoscere intrecci, peculiarità, situazioni e percorsi che hanno intessuto i tanti fili del fumetto contemporaneo. Un mondo complesso, articolato, ancora in gran parte da scoprire e da capire per gli storici; che muove passioni e crea miti, si presta a strumentalizzazioni propagandistiche e a banalizzazioni didattiche, ha a che fare con interessi economici rilevanti, coinvolge nella lettura milioni di persone, mostra una notevole capacità di adattamento al trasformarsi delle tecniche per la produzione artistica e dei supporti per il consumo dei fumetti, su carta o schermi di varie forme e dimensioni.

Roberto Bianchi

Thierry Smolderen

Naissances de la bande dessinée.

De William Hogarth à Winsor

McCay

Les Impressions Nouvelles,

[Bruxelles] 2009, pp. 143, ill.

Il belga Thierry Smolderen, sceneggiatore di bande dessinée e docente preso la École des Beaux-Arts di Angoulême, è uno dei maggiori esperti di quella che oggi gli americani indicano come Victorian Age del fumetto, e che si riferisce agli anni anteriori al 1896. Inoltre, come ispiratore del sito web Coconino World, organizzatore di mostre e divulgatore, è uno dei principali animatori nel dibattito attuale sulle origini del medium. In sintonia con le recenti acquisizioni offerte dagli studi, l'A. fa muovere il suo saggio più recente dal presupposto che la storia del fumetto affondi le proprie radi- 
ci nelle stampe popolari del ' 700 , emerga come forma consapevole nella prima metà del secolo successivo e si imponga come industria culturale di massa nell'ultimo quarto dell'800 in Gran Bretagna, Francia e Germania, fino al boom americano negli anni che precedono il secolo breve.

Tale sintesi si basa su un ripensamento della storia del fumetto, che negli ulti$\mathrm{mi}$ anni ha cambiato in profondità i propri strumenti di analisi e le proprie premesse. Gli anni '60 del secolo scorso avevano infatti visto nascere, fra Stati Uniti ed Europa, una critica specializzata che, impegnata nella ricostruzione di un repertorio di personaggi e autori, ma anche di pubblicazioni, assegnava un esplicito ruolo discriminante alla industrializzazione dei processi culturali nella società americana, identificando l'origine del genere nel 1896, con l'apparizione di un balloon nelle storie del personaggio Yellow Kid. Questa genealogia era legata anche al recupero nostalgico del fumetto anni '30, caratteristico del periodo, e affondava le radici in alcuni lavori pionieristici: in particolare The Comics di Coulton Waugh (The MacMillan Company, New York 1947), esplicito punto di riferimento per almeno il primo decennio di studi nel settore.

Ciò non toglie che anche all'epoca fossero conosciuti gli antecedenti del «romanzo grafico»: due esempi legati al ginevrino Rodolphe Töpffer, oggi riconosciuto come uno dei padri del medium nella prima metà dell' 800 , sono stati l'antologia curata da Ellen Weiss (Enter: The comics, University of Nebraska Press, Lincoln 1965) e l'edizione Garzanti delle opere apparsa nel 1973. Ma la stessa qualifica di "precursori" del fumetto, usata spesso dalla critica, sminuiva il ruolo di questi autori, implicitamente invitando a tralasciarne le esperienze (cfr. Fabio Gadducci e Matteo Stefanelli, La storiografia del fumetto in Italia. Tradizione e strategie culturali, in Il secolo del fumetto. Lo spettacolo a strisce nella società italiana 1908-2008, a cura di Sergio Brancato, Tunuè, Latina 2008, pp. 106-30).

Un punto di svolta si può assegnare all'uscita del volume di David Kunzle sulla History of comic strips (University of California Press, Berkeley 1973) che ha il pregio di identificare temi e tecniche di narrazione per immagini presenti sin dalle origini nelle stampe popolari. Lo scatto successivo si può far risalire al 1996: il centenario del balloon di Yellow Kid offre l'occasione per una decisa ripulsa del "mito delle origini", esemplificata da una collezione di saggi curata da Charles Dierick e Pascal Lefèvre (Forging a new medium, Vub UP, Bruxelles 1998), la cui prefazione A Timeline, 1800-1914 già identifica i limiti temporali, e dunque metodologici, dell'operazione. Da allora, una generazione di studiosi ha approfondito gli spunti di lavoro emersi dagli interventi raccolti nel volume, mentre sono stati riportati alla luce molti materiali "dimenticati", anche grazie a mailing list come PlatinumAgeComics e a siti web come Early Comics di Andy Bleck (konkykru.com).

Coerente con questo assunto, Smolderen passa in rassegna quelli che appaiono i nuovi capisaldi del genere, dalle stampe di William Hogarth nella seconda metà del ' 700 agli album di Töpffer, ai successivi sviluppi europei e americani veicolati dalla stampa periodica, per terminare con una ricognizione su due autori ritenuti capitali nel percorso del fumetto, gli americani Arthur Burdett Frost e Winsor McKay. A supporto del proprio argomentare l'A. rende disponibile nel volume, profusamente illustrato, un'ampia serie di testimonianze grafiche di difficile reperibilità. Fra queste sono compresi anche materiali eterogenei, quali i repertori ottocenteschi di espressioni facciali al servizio degli attori, che l'analisi di Smolderen recupera alla storia del $m e$ dium.

Il volume non presenta comunque una ricostruzione storica lineare. Al contra- 
rio, la sua riflessione parte da un assunto ben diverso, indicato nel titolo dalla scelta del plurale naissances: l'idea di molteplici nascite, di più momenti storici che hanno contribuito a definire quella forma indicata oggi come "fumetto", e che nel corso dello sviluppo si sono certo intrecciati, ma anche reciprocamente elisi. Se vogliamo, proprio questa intuizione rappresenta il punto più stimolante del volume: l'A. mette in guardia dall'adottare negli studi storici sul fumetto una definizione diacronica del genere, utilizzandola come discrimine nel tentativo di identificare quali siano state le opere che ne hanno influenzato la formazione. Il difetto delle ricostruzioni troppo mirate è emerso con chiarezza per esempio negli scorsi anni '70 e '80, quando la riflessione semiologica aveva identificato nella nuvoletta la caratteristica fondante del fumetto, con il risultato di rendere inintelligibili larghi segmenti della sua evoluzione, in particolare per quel che riguarda la realtà italiana, dominata nei primi decenni del '900 dagli ottonari del «Corriere dei Piccoli». Di fronte a questo, l'intuizione di Smolderen, ulteriormente elaborata e resa esplicita in un saggio più recente (A chapter on methodology, «Signs-Studies in Graphic Narratives», suppl. 2, 2010), rappresenta senza alcun dubbio il contributo metodologico più rilevante dell' $A$. al campo di studi.

Come c'è da attendersi, tuttavia, non tutte le interpretazioni che il volume offre sono da considerarsi assodate. Questo è particolarmente vero per i capitoli su Töppfer, che l'A. considera un fondatore «riluttante» del medium: lungi dall'esser frutto dell'impegno di un convinto assertore delle possibilità espressive della narrazione disegnata, i lavori dell'artista ginevrino rappresenterebbero un'ironica presa di distanza dal concetto stesso di "azione progressiva", dunque sequenziale, che a metà ' 700 era stata propagandata da Gotthold Lessing come tecnica stilistica d'elezione per la poesia. Opinione ribadita da Smolderen anche in Töpffer
Reloaded. A revaluation of Töpffer invention («Signs-Studies in Graphic Narratives», 2, 2011, pp. 53-62), e che ha dato origine a un lungo scambio epistolare dello stesso con Thierry Groensteen, che da tempo rivendica invece la consapevolezza propositiva dell'invenzione töpfferiana (come nel saggio con Benoit Peteers, Töpffer. L'invention de la bande dessinée, Hermann, Paris 1994).

La struttura leggera del volume non permette di approfondire tutti i temi che vengono proposti, ma è certo una scelta dell'A. quella di aver lasciato in ombra l'influenza che ha avuto nell'evoluzione del fumetto lo sviluppo tecnologico dei mezzi di comunicazione. Un esempio riguarda il concetto stesso di nuvoletta: espediente grafico che, lungi dall'essere un'invenzione di fine ' 800 , è ben presente già nelle stampe popolari inglesi. Smolderen dedica all' argomento un breve capitolo, quasi un inciso nello svolgimento della sua analisi, rielaborando un precedente intervento (Of labels, loops, and bubbles: Solving the historical puzzle of the speech balloon, «Comic Art», 8, 2006, pp. 90-112). La sua proposta di distinzione fra "etichetta", priva di una componente dialogica, e vera e propria "nuvoletta", reintrodotta a fine '800 come strumento per rendere la scansione spaziale e temporale degli avvenimenti, per quanto affascinante, non spiega però compiutamente la presenza di usi esplicitamente dialogici nelle stampe settecentesche. Lascia inoltre in disparte l'influenza che la riproduzione litografica, la tecnica per eccellenza nei periodici ottocenteschi, ha avuto nel far preferire al balloon l'inserimento tipografico del testo in calce alle vignette.

Il volume si presenta dunque, e lo stesso A. lo ammette, come una preliminare mappatura del campo, una sorta di "lavori in corso": ed è, in quanto tale, uno dei contributi più promettenti e stimolanti di questi ultimi anni. Lo scopo della ricerca di Smolderen, ovvero l'identificazione per il fumetto di una de- 
finition with an historical domain, ha il pregio di restituire una componente temporale allo sviluppo del medium, calandolo nella temperie culturale degli anni che ne hanno visto la nascita (o le nascite) e la diffusione, in tal modo rendendolo infine "materia" di Storia.

Fabio Gadducci

Vincent Marie-Historial de la Grande Guerre (sous la direction de)

La Grande Guerre dans la bande dessinée de 1914 à aujourd'hui

5 Continents Editions, Milano 2009, pp. 111, ill.

\section{Fabiana Loparco}

I bambini e la guerra.

Il Corriere dei Piccoli e il primo

conflitto mondiale (1915-1918)

Nerbini, Firenze 2011, pp. 204

Studiare la storia delle rappresentazioni della Grande Guerra nei fumetti prodotti in Italia e Francia significa confrontarsi con una tipologia di fonti il cui studio ha seguito percorsi e prodotto risultati diversi fra i due paesi.

Oltralpe il rapporto tra bande dessinée e storia, la storia nella $B D$ e della $B D$, sono stati oggetto di molte ricerche condotte da storici - francesi o di area francofona -, tanto che si può parlare di una presenza della $B D$ nella storiografia. In Italia questa presenza è assai più rarefatta. Il tema è stato preso in considerazione da semiologi, linguisti, pedagogisti, studiosi delle comunicazioni di massa, con approcci utili ma privi tuttora di una capacità di sintesi e dialogo tra diverse discipline. In particolare, la storiografia ha sottovalutato a lungo fonti che invece possono dirci molto sui processi di costruzione delle forme di propaganda bellica, sui tentativi e gli esiti dei progetti di nazionalizzazione delle masse, sui rapporti tra Stato, mercato editoriale e società mobilitate o smobilitate nei dopoguerra del '900. Va detto che le ricerche più recenti sull'infanzia e la gioventù, sulla nazionalizzazione del popolo bambino, su periodici come il «Corriere dei Piccoli» o sul rapporto tra fumetti e fascismo, indicano una decisa controtendenza. Eppure, per fare un esempio, nelle 14 "isole tematiche" della ricca, affascinante e, in tutta evidenza, impegnativa mostra Fare gli italiani. 150 anni di storia nazionale, realizzata a Torino nel 2011 (cfr. i contributi di Massimo Baioni e Maurizio Bertolotti in «Passato e presente», 86, 2012, pp. 83-93 e 95-105), si poteva trovare di tutto; ma non si riusciva a scovare nemmeno un fumetto.

Questa differenza di statuto per i fumetti fra i due paesi era stata evidente anche in occasione del $90^{\circ}$ anniversario della prima guerra mondiale. Se in Francia furono realizzate mostre, pubblicati cataloghi, organizzati convegni ed eventi sulle rappresentazioni della guerra nei fumetti, in Italia mostre, cataloghi ed eventi - che pure ci furono - ebbero una dimensione più modesta, comunque non paragonabile alle iniziative organizzate, per esempio, all'Historial de la Grande Guerre de Péronne dove, per dire, nell'estate $2009 \mathrm{fu}$ invitato Jacques Tardi, uno dei più importanti e discussi autori di $B D$ sul tema.

La diversità è dovuta a vari fattori che qui non si possono esaminare, in ogni caso non dipendenti dalle storiografie nazionali sulla Grande Guerra, ma piuttosto dal più generale livello di considerazione per i fumetti da parte delle comunità scientifiche e dalle caratteristiche dei diversi mercati editoriali di fumetti e $B D$. È probabile che questo squilibrio tenderà finalmente a colmarsi in vista del centenario del 1914-18, come sembrano indicare notizie che giungono da alcuni blog di autrici e autori italiani, e sapendo di tesi e ricerche in corso, condotte da studiosi il più delle volte giovani. Una distanza che il volume di Fabiana Loparco, come la mostra trentina su La Grande guerra del Corriere dei Piccoli 19141919 - curata da Nicola Spagnolli e inaugurata mentre sto scrivendo - contribuiscono a ridurre. 
Il lavoro di Loparco gravita intorno all'analisi di fumetti, rubriche, testi e racconti pubblicati dal «Corriere dei Piccoli» tra l'estate 1914 e l'inizio del 1919 , in un libro che - citando spesso, a giusto titolo, Antonio Gibelli - si apre con una descrizione del panorama di giornalini patriottici impegnati nella nazionalizzazione dell'infanzia a cavallo tra '800 e '900, per poi dedicare un intero capitolo al "Corrierino" durante la guerra di Libia. Nel più ampio capitolo centrale, il terzo, sono lette le pagine del periodico negli anni della mobilitazione bellica, con un paragrafo per ognuna delle saghe illustrate prodotte tra l'intervento e la disfatta di Caporetto (Schizzo, Luca Takko e Gianni, Italino, Tofoletto pancia vuota, l'epistolario a Franz Joseph, Abetino e Didì, l'eroina "al femminile"), da artisti come Antonio Rubino, Attilio Mussino, Gustavo Rosso, Mario Mossa de Murtas. Più breve il quarto capitolo, dedicato agli stretti rapporti fra gli autori del settimanale, il Servizio P e i giornali di trincea nell'ultimo anno di guerra, prima di quello finale sul «Corriere dei Piccoli»e la pace, che getta uno sguardo anche sull'immediato dopoguerra. Nonostante alcune sviste - ad esempio, poteva essere ricordato il legame, evidenziato da $\mathrm{N}$. Spagnolli nella mostra citata, tra l'esecuzione di Cesare Battisti, illustrata da Achille Beltrame sulla copertina della «Domenica del Corriere» il 30 luglio 1916 , e la distruzione della forca che doveva impiccare il vincente Italino nell'episodio del 22 ottobre 1916 -, nel complesso si tratta di una ricerca lodevole, basata sulla rielaborazione della tesi di laurea, che pone alcuni punti fermi importanti e apre prospettive per successivi approfondimenti.

Il volume curato da Vincent Marie è invece una raccolta di saggi prodotti in occasione delle mostre Tardi e Mobilisation générale! 14-18 dans la bande dessinée, tenute all'Historial nel 2009. Contributi sulle pubblicazioni francesi più recenti (dalle opere di Tardi a Le Coeur des Batailles di Jean-David Morvan e Igor Kordey) o più distanti nel tempo (Bécassine e Les Pieds Nickelés, all'epoca comparse su «La Semaine de Suzette» e «L'Épatant», poi raccolte in albi), lette con cura e aggiornati strumenti analitici, si alternano a utili riflessioni sul percorso storico di iconografie, mitologie, forme di rappresentazione (ad esempio del "nemico") presenti nelle $B D$, prima di dedicare spazio, con due interventi, ai fumetti tedeschi e anglosassoni. Va segnalato che il libro si chiude con una bibliografia dei fumetti sulla Grande Guerra pubblicati tra 1914 e 2009 , che avrebbe potuto essere uno strumento utile. Esplicitamente non esaustivo, l'elenco è comunque troppo lacunoso e organizzato in modo discutibile. Non si riesce a capire, ad esempio, perché la prima sezione riunisca assieme i fumetti franco-belgi con quelli italiani - la seconda e la terza sezione sono dedicate, rispettivamente, a una decina di prodotti anglosassoni e a 4 tedeschi -, suddividendo i 114 titoli prescelti in sottosezioni cronologiche, con periodizzazioni dipendenti dalla produzione francofona (oltre ad alcune di Pratt, ho rintracciato nell'elenco opere di appena 4 autori italiani: D'Antonio, Micheluzzi, Tacconi e Toppi, nelle loro traduzioni francesi).

In ogni caso, le chiavi di lettura e le informazioni fornite dai saggi risultano molto utili, e si possono anche confrontare con quelle pubblicate in altre opere sul fumetto francese (penso, ad esempio, al numero monografico su Tardi di «Sociétés \& represéntations», n. 29, 2010, a cura di Bertrand Tillier, o al volume di Bruno Denéchère e Luc Révillon, 14-18 dans la bande dessinée. Images de la Grande Guerre de Forton à Tardi, Cheminements, Turquant 2008).

Inizia, dunque, a essere possibile tracciare un'analisi comparativa sulle forme di rappresentazione della Grande Guerra tra $\mathrm{i}$ due paesi, anche se resta da fare molta ricerca su periodici e albi non ancora pesi in considerazione. Agli ele- 
menti comuni tra la produzione francese e italiana, che inducono a individuare una vera e propria nascita dell'Europa proprio con la prima grande mobilitazione morale e intellettuale dell'infanzia nella sfera politica europea (cfr. A. Gibelli, Il popolo bambino, Einaudi, Torino 2005, p. 41; S. Audouin-Rouzeau, La Guerre des enfants 1914-1918, Armand Colin, Paris 1993, p. 186), vanno però affiancate e lette criticamente le importanti differenze tra i due paesi. Anche guardando ai soli anni del conflitto, non possiamo ignorare le ricadute sulla produzione artistica e sul mercato editoriale della minore nazionalizzazione delle masse italiane rispetto a quelle francesi, al momento di un intervento tanto contrastato quanto voluto solo da una parte del paese.

Anche per questo è importante analizzare il «Corriere dei Piccoli», assai diffuso fin dagli anni ' 10 e probabilmente più letto di libri come Cuore o Pinocchio (stando ai dati forniti da M. Fiore, $L a$ lettura dei "piccoli". Un'indagine del 1912, «La Fabbrica del Libro», 1, 2005, pp. 33-38, relativi al caso di Bologna). Anche perché le sue pagine imposero un modello, una tipologia di fumetti che in Italia avrebbe dominato a lungo e che già durante la guerra si riversò nei giornali di trincea, assieme all'esperienza di una generazione di fumettisti trasferita direttamente dai giornalini alle redazioni de «La Tradotta» o delle altre decine di periodici concepiti per soldati poco alfabetizzati e ancor meno nazionalizzati.

Eppure non basta esaminare il «Corrierino» o «La Semaine de Suzette» se vogliamo capire i percorsi che, attraverso i fumetti "classici" post crisi del 1929 e i periodici di metà ' 900 , portano al profondo rinnovamento della prima guerra mondiale nei fumetti tra gli anni '60 e '70, prima di giungere alle complessità e alle varietà di temi e forme di rappresentazione grafica adesso presenti nelle $B D$ dei due paesi, a cent'anni di distanza dal grande conflitto che segnò una cesura irreversibile anche per la Nona arte.

Roberto Bianchi

Giovanna Ginex (a cura di)

Corriere dei Piccoli.

Storie, fumetto e illustrazione

per ragazzi

Skira, Milano 2009, pp. 318, ill.

Renata Lollo (a cura di)

Il «Corriere dei Piccoli» in un secolo

di riviste per ragazzi

Atti del convegno (Università

Cattolica del Sacro Cuore, Milano,

28 marzo 2008)

Vita e Pensiero, Milano 2009, pp. 170, ill.

Entrambi i libri sono frutto delle celebrazioni per il centenario della nascita del «Corriere dei Piccoli», avvenuta a Milano il 27 dicembre 1908; un avvenimento importante sia per la storia della letteratura per l'infanzia, sia per quella del fumetto. I due volumi - letture imprescindibili per comprendere la storia del celebre giornalino per l'infanzia raccolgono un'ampia serie di saggi dedicati a molteplici e diversi aspetti del «Corrierino»: l'analisi delle illustrazioni, dei singoli personaggi, della direzione della rivista e delle politiche editoriali, dei vari collaboratori e disegnatori concorrono a costruire un quadro organico che restituisce a lettori e lettrici di oggi le atmosfere e lo stile di lavoro che regnavano nella redazione del «Corriere dei Piccoli».

Il libro curato da Giovanna Ginex nasce da una mostra organizzata dalla "Fondazione Corriere della Sera" di Milano ed è sorretto da una corposa galleria d'immagini reperite presso l'importante archivio del «Corriere della sera». Di notevole livello è il saggio della curatrice che, con un accurato uso delle lettere e di altri materiali presenti nell'archivio della Fondazione, traccia un quadro inedito e approfondito della genesi del gior- 
nalino (dagli albori, con la collaborazione di Paola Lombroso Carrara e l'uso dei cliché inglesi e americani), fino a descrivere dettagliatamente le collaborazioni dei principali illustratori e i loro rapporti, non sempre idilliaci, con la redazione. $\mathrm{Al}$ contempo, i contributi di Antonio Faeti, Giulio Cesare Cucciolini, Gianni Brunoro e Silvia Magistrali tracciano la storia del periodico con sguardo attento e onnicomprensivo, dal primo numero del 27 dicembre 1908 all'ultimo del 15 agosto 1995. Va detto che questi autori si soffermano soprattutto sull'analisi delle illustrazioni, elemento di modernità, nonché di indiscutibile fascino e attrazione grazie all'uso di colori sgargianti, alla magistralità del tratto e alla briosità dei temi trattati, senza però dimenticare di fornire anche analisi e descrizioni dei personaggi comparsi sul giornale prima e dopo la seconda guerra mondiale. Interessante il saggio di Antonello Negri e Marta Sironi, che mette in relazione la storia del «Corriere dei Piccoli» con quella, ancora in gran parte da scoprire, della stampa periodica per l'infanzia nata in Italia sul finire dell'800: il giornale antesignano per eccellenza fu, infatti, «Il Giornale per i Bambini» fondato a Roma nel 1881 da Ferdinando Martini, primo esempio del connubio "istruire divertendo", che sarebbe stato fatto proprio anche dal «Corriere dei Piccoli» sotto la direzione di Silvio Spaventa Filippi.

Il volume si chiude con tre testimonianze. La prima, di Grazia Nidasio, presenta una straordinaria carrellata di direttori che hanno tenuto vivo per ben 87 anni il periodico: 16 direttori e un «fantasma», scrive la Nidasio, che non dimentica di ben illustrare l'importantissimo e misconosciuto lavoro svolto da Paola Lombroso Carrara per ideare e dar vita al giornalino di via Solferino. Ritratti brevi e incisivi, con quei protagonisti tratteggiati nei loro aspetti professionali e umani a volte con un filo di ironia e nostalgico ricordo, tracciando il quadro di una redazione mutevole e statica allo stesso tem- po, capace di innovarsi e aprirsi alle novità non mancando, però, di rispettare le proprie "tradizioni" (come altro interpretare, ad esempio, la «richiamata alle matite» fatta da Mosca negli anni del dopoguerra a Rubino o Tofano?). Con Alfredo Castelli, invece, si entra in contatto con quel «Corriere dei Ragazzi» al centro, ancora oggi, di giudizi contrastanti. Frutto di uno spirito innovatore che nei primi anni '70 spinse la redazione a cercare di rivolgersi non più solo ai bambini ma anche agli adolescenti, il nuovo periodico si aprì alla pubblicazione di tavole e storie rimaste poi nella memoria (come Corto Maltese di Hugo Pratt, Valentina Mela Verde della stessa Nidasio o i Caroselli del Calimero di Sergio Toppi), e presentò ai lettori, con un certo coraggio, racconti dedicati a temi e problemi di attualità, vicende e personaggi "scomodi" o complessi, prima ritenuti poco adatti alla fruizione di un pubblico infantile, ma che invece il "Corriere dei Ragazzi» poté proporre agli adolescenti, instaurando con essi un rapporto paritario e costruttivo. Un ultimo ricordo è affidato ad Alfredo Barberis, il quale ripercorre il suo lavoro come direttore del nuovo «Corrierino» descrivendo con delicatezza e affetto staff e personaggi (in particolare la celebre Pimpa e il «terrone» Gennarino Tarantella) e mostrando come il giornale sia stato luogo di transizione per artisti come Alberto Moravia, Mino Milani o Tiziano Sclavi.

Il libro della Lollo invece raccoglie i contributi presentati al convegno organizzato presso l'Università Cattolica di Milano il 28 marzo 2008 per celebrare il centenario del «Corrierino». Il volume si apre con un intervento di Giorgio Chiosso, capace di fornire il quadro storicopubblicistico entro cui nacque e si sviluppò l'iniziativa del «Corriere dei Piccoli», attraverso uno sguardo attento al ruolo della stampa per maestri e allievi. Segue un'analisi dei due personaggi che più determinarono il carattere del «Corriere dei Piccoli»: Paola Lombroso Carrara e 
Silvio Spaventa Filippi. Alla Lombroso, in particolare, sono dedicati due saggi che ne tracciano l'importante attività al «Corrierino»; Alberto Carli ne ricostruisce il profilo biografico, presentandola come una donna straordinaria, attenta alle tradizioni ma aperta alle innovazioni, conscia del proprio ruolo di educatrice dell'infanzia in nome di un processo di rinnovamento di tutta la società. Lo stesso spirito innovativo-educativo, d'altronde, ben emerge nel saggio di Lorenzo Cantatore dove si fa luce sulla corrispondenza tenuta da Paola Lombroso sulle pagine del «Corrierino» con lo pseudonimo di Zia Mariù: una corrispondenza volta a instaurare un rapporto vivo con il suo pubblico di lettori, convinta che il motto "insegnare divertendo" fosse l'unica strada percorribile per educare e nazionalizzare le giovani generazioni.

Sabrina Fava, invece, approfondisce la figura di Spaventa Filippi; uomo colto e discreto, fu il primo in Italia a presentare racconti e fumetti provenienti da paesi stranieri dando al giornale un respiro più ampio e meno provinciale, accostando agli apporti internazionali quelli dei migliori illustratori e scrittori italiani del periodo, sullo sfondo di un progetto culturale che coniugava fantasia ed esigenze educative, il bisogno di svago e divertimento dei lettori con la formazione di una solida coscienza civile e nazionale. Fu Spaventa Filippi che, nel 1917, spinse per la creazione del signor Bonaventura, disegnato da Sergio Tofano e fatto rivivere nelle pagine del libro dal saggio di Ilaria Filograsso. Popolarissimo e amatissimo, il signor Bonaventura divenne presto simbolo dell'Italia povera che sognava la ricchezza, maschera delle aspirazioni e apprensioni di una fiduciosa umanità.

L'unico sguardo al «Corriere dei Piccoli» in età "avanzata" è fornito dal contributo di Fausto Colombo, che ripercorre le tappe della nascita del personaggio di Valentina Mela Verde, comparsa sul «Corrierino» nel 1968 dalla matita di
Grazia Nidasio e trasmigrato, poi, sul «Corriere dei Ragazzi» nel 1972. Personaggio innovativo, Valentina è una ragazza comune che vive le novità del suo tempo: il pacifismo, le contestazioni, i viaggi, i primi innamoramenti. Divenne simbolo di una nuova generazione di ragazze pronte al rinnovamento, per quanto la Nidasio rimanesse sempre attenta a contenere i fermenti innovatori entro l'alveo sicuro del nucleo familiare, ancora presentato come l'unico luogo al cui interno - precisa Colombo - «valesse ancora la pena di crescere» (p. 139).

Infine, il saggio di Mirella Ferrari, dedicato alla storia della stampa periodica per ragazzi sviluppatasi nell'ambito dell'Università Cattolica di Milano, è in grado di richiamare nomi ed esperienze che ben dimostrano come la lezione del «Corriere dei Piccoli» sia stata un modello imprescindibile, un punto di riferimento per tutta la stampa successiva.

Nel loro insieme, i due volumi riescono a dare un taglio critico e scientifico a un argomento come quello dei fumetti e della stampa periodica per l'infanzia spesso bistrattato, lasciato ai margini del mondo accademico e letterario perché ritenuto troppo "infantile" e "massificato". Il convegno, la mostra, le pubblicazioni sono invece il segno di cambiamenti importanti, nel solco di una sempre più massiccia serie di studi multidisciplinari realizzati da studiosi e studiose che concorrono a dare consistenza e dignità a un ambito di ricerca di indubbio rilievo.

Fabiana Loparco

Fabio Gadducci-Leonardo Gori-

Sergio Lama

Eccetto Topolino.

Lo scontro culturale tra il fascismo

e il fumetto

Nicola Pesce Editore, Roma 2011,

pp. 431, ill.

La novità di questo volume non sta tanto nel tema trattato, già oggetto di un lungo ed intenso dibattito all'interno del- 
la storia del fumetto italiano a partire dagli anni ' 70 del secolo scorso (qui arricchito di numerosi nuovi elementi e di vere e proprie rivelazioni), quanto nel metodo d'indagine utilizzato dagli autori. La storia del fumetto, infatti, almeno nel nostro paese, è stata per lungo tempo appannaggio più di consumati collezionisti e appassionati cultori della materia che non degli storici, nonostante incursioni più $\mathrm{o}$ meno episodiche siano state tentate da parte di studiosi provenienti da varie specialità (come la storia dell'editoria e della stampa, la storia dei processi culturali e mediatici, la storia dell'illustrazione o la storia della letteratura per l'infanzia). Una storia, quella del fumetto italiano, scritta dunque per lo più da "non professionisti", con evidenti per quanto giustificabili lacune metodologiche, dal mancato confronto con la più ampia letteratura scientifica alla sostanziale incapacità di sottoporre le fonti a un'esegesi accurata e rigorosa. Ma questa non è stata l'unica conseguenza negativa di questo "malcostume storiografico", che proprio a causa del ruolo primario assunto da appassionati e collezionisti, per loro stessa natura interessati alla storia (o forse più riduttivamente alle vicende) delle testate, degli autori e dei personaggi più celebri - ha provocato una sensibile polarizzazione degli studi intorno agli "anni d'oro" del fumetto, ovvero quegli anni ' 30 che videro il dilagare in tutto il paese di albi e giornaletti i cui eroi prediletti provenivano nella loro quasi totalità d'oltreoceano e che proprio per questo erano stati sottoposti a una rigida censura da parte dell'esterofobo regime fascista, divenendo così introvabili pezzi da collezione. Per questo motivo, in definitiva, «lo scontro culturale tra fascismo e fumetti» oggetto del presente volume è già stato analizzato da molti altri autori (mi limiterò qui a fare riferimento a La stampa periodica per ragazzi: da "Cuore" a Charlie Brown di G. Genovesi (Guanda, Parma 1972), a Il fascismo a fumetti di C. Carabba (Guaral- di, Firenze 1973) e a Fumetto alalà: $i$ comics italiani d'avventura durante il fascismo di G. Pazienti e R. Traini (Comic Art, Roma 1986), i quali hanno alimentato nel corso del tempo un'inesauribile sequela di rimandi incrociati gli uni con gli altri, contribuendo a perpetuare errori, luoghi comuni e pretesi arcani che questo volume contribuisce in parte a destituire definitivamente di ogni fondamento. Uno di questi arcani è l'eccetto Topolino che gli autori utilizzano come titolo per la loro opera: la citazione è tratta da una circolare emanata nel 1938 dal ministro della Cultura popolare Dino Alfieri, con la quale si aboliva una volta per tutte il materiale fumettistico d'importazione straniera «fatta eccezione per le creazioni di Walt Disney, che si distaccano dalle altre per il loro valore artistico e per la sostanziale moralità». Sulle reali ragioni di questa eccezione già in molti si sono interrogati nel recente passato; uno fra tutti Francesco De Giacomo, la cui nota intervista a Romano Mussolini (qui integralmente riprodotta) aveva contribuito nel 1995 a rilanciare il banalizzante mito - già accreditato negli ambienti fumettistici - di un duce bambinescamente infatuato di Topolino e delle produzioni Disney, anche sulla scia del forte ascendente su di lui esercitato dai figli Vittorio (che aveva eseguito nel 1937 un trionfale viaggio negli States, durante il quale aveva conosciuto anche Walt Disney) e Annamaria, che era un'assidua lettrice di «Topolino» e che come ricordava nel 1958 Rachele nelle sue memorie - durante la sua malattia aveva ricevuto da Walt Disney in persona un Mickey Mouse gigante (F. De Giacomo, Quando il Duce salvò Topolino, «IF. Immagine e Fumetti», 4, 1995, pp. $8-15$; l'intervista ebbe una notevole eco all'epoca e i suoi contenuti furono ripresi anche dal «Corriere della sera» e «la Repubblica», $1^{\circ}$ ottobre 1995 ).

Gli autori di questo volume tornano a interrogarsi su quell'eccezione, ritenuta paradigmatica delle complesse vicende 
editoriali legate alla straordinaria diffusione dei fumetti americani nell'Italia degli anni '30, fornendo altre risposte, meno legate all'umore del duce (che pure - come testimonia anche una recente biografia di Walt Disney curata da $\mathrm{M}$. Barrier - ricevette personalmente i fratelli Disney a Palazzo Venezia nell'estate 1935 ed era personalmente legato all'editore americano Hearst, al quale faceva capo il King Features Syndicate, che deteneva i diritti di riproduzione di tutti i fumetti americani) e più agli enormi interessi economici di una casa editrice come Mondadori, saldamente legata al regime, che aveva acquisito i diritti per la riproduzione delle pubblicazioni disneyane in Italia. La chiave di volta che ha consentito a questo studio di svelare le reali ragioni di quell'eccezione è stata la "scoperta" presso la Fondazione «Franco Fossati» a Milano e lo spoglio analitico delle carte private di Guglielmo Emanuel, corrispondente dell'agenzia di stampa americana International News Service e rappresentante per l'Italia del King Features Syndicate. Le carte di Emanuel, infatti, combinate con quelle reperite presso l'archivio storico della Arnoldo Mondadori Editore a Milano e le altre - istituzionali - conservate in parte nell'archivio della Prefettura di Cremona presso il locale Archivio di Stato e in parte nell' archivio del ministero della Cultura popolare presso l'Archivio centrale dello Stato a Roma, hanno consentito agli autori di documentare efficacemente il complesso intreccio di interessi economici che aveva permesso al «Topolino» della Mondadori di uscire indisturbato nelle edicole tra il 1938 e il 1942, mentre la censura fascista si abbatteva implacabile sugli altri periodici a fumetti, così come anche di gettare nuova luce sulle dinamiche editoriali che avevano sovrinteso alla pacifica invasione dei fumetti americani a partire dalla prima metà degli anni '30, relativamente alla quale sembrava ormai che davvero poco rimanesse da dire.
Eppure questo volume, che costituisce in qualche misura la prova indiscutibile della maturità storiografica alla quale è giunta (o sa giungere) la storia del fumetto oltre che della competenza raggiunta da storici "non professionisti", lascia ancora in ombra alcuni aspetti dello «scontro culturale tra fascismo e fumetti». Per l'ennesima volta, infatti, si insiste qui più sulle ragioni - ormai acclarate - della violenta campagna persecutoria del fascismo nei confronti dei comics americani e sul "disagio" dimostrato nei confronti della stessa da editori e commercianti che vedevano lesi i propri interessi in virtù della loro pretesa adesione ai valori dell'etica fascista, piuttosto che sugli esiti che quella campagna stessa riuscì effettivamente a produrre tra i lettori (divennero essi "più fascisti"?, oppure si disaffezionarono progressivamente a un regime che boicottava le avventure dei loro eroi?). Nonostante che in calce al volume sia riportata una pratica appendice documentaria con le circolari emanate dal ministero della Cultura popolare in materia di stampa per ragazzi tra il 1941 e il 1943, rimane ancora da chiarire il ruolo, il funzionamento e i risultati effettivamente raggiunti dall'Ufficio di revisione della letteratura giovanile (istituito nel 1942 presso il Minculpop, con apposite commissioni distaccate a livello provinciale presso tutte le prefetture) nella lotta contro la «stampa nociva», che durante il suo primo anno di vita aveva approvato senza osservazioni il $35 \%$ delle opere ad esso sottoposte, accolto con opportune modifiche il $34 \%$ e respinto il restante $31 \%$ (Bonifica della letteratura giovanile, «I diritti della scuola», n. 8, 10 dicembre 1942, pp. 140-41). Poter leggere oggi i fumetti - o anche solo i loro storyboard - che il fascismo non volle far leggere ai propri "figli", ovvero avere accesso agli "scarti" del procedimento censorio, infatti, consentirebbe di disvelare ulteriormente il quadro dello «scontro culturale tra fascismo e fumetti».

Juri Meda 
Vicent Sanchis

Franco contra Flash Gordon.

La censura franquista aplicada a les publicacions infantils i juvenils (1936-1977)

Edicions Tres i Quatre, Valencia 2009, pp. 231, ill.

Lo sviluppo della saggistica spagnola dedicata al fumetto ricorda da vicino quello della contemporanea esperienza italiana. Si fonda attraverso gruppi di appassionati nel corso degli anni '60 - fra i quali citiamo almeno, per l'influenza avuta nel nostro paese, Luis Gasca (collaboratore di «Comics Club 104», prima rivista amatoriale in Italia dedicata al fumetto) e Roman Gubern, fondamentale da noi nel settore degli studi semiotici applicati al fumetto -, e matura con la produzione del testo "canonico" di Antonio Martin, Historia del comic español, 1875-1939 (Editorial Gustavo Gili, Barcelona 1978), che al passo con la storiografia di quegli anni offre una ricostruzione di lungo periodo. La riflessione attraversa un momento di stanca negli anni ' 80 , dal quale si riprende nel corso del decennio successivo, quando il fumetto comincia a essere oggetto d'indagine sia come fenomeno editoriale che culturale, fino ad arrivare alla divulgazione colta del percorso storico dei fumetti, come mostra la recente serie Del tebeo al manga: 12 volumi riccamente illustrati, pubblicati dalla filiale locale della Panini e curati dal critico Antoni Guiral.

Il volume di Sanchis, dunque, si inserisce in un rinnovato interesse per la storia dell'editoria a fumetti affrontando lo specifico della censura franchista nelle pubblicazioni per l'infanzia e la gioventù, ma includendo, in realtà, anche il periodo monarchico e repubblicano. Per esemplificare il portato delle politiche governative, l'A. sceglie come cartina di tornasole Flash Gordon, personaggio che, in virtù della sua lunga permanenza sul mercato (fra prime edizioni e ristampe) si presta per capire sia le modalità di esecuzione che le spinte ideologiche del- la legislazione imposta alla stampa per ragazzi durante la dittatura.

L'avventura editoriale di Gordon attraversa quasi un quarantennio di storia spagnola. La prima traduzione avviene nel 1936-38, in piena guerra civile, sul settimanale «Aventurero», ispirato all" «Avventuroso». Quasi una copia, in effetti, del periodico italiano: lo edita la barcellonese Hispano Americana de Ediciones, che è in realtà la filiale spagnola della casa editrice Vecchi, protagonista di primo piano dell'avvento del fumetto moderno in Italia, che cerca di bissare il successo del periodico pubblicato a Firenze da Nerbini. La stessa casa editrice pubblica due serie di albi ( 9 nel 1942-44 e ulteriori 18 a fine decennio) che includono tutto il periodo classico del personaggio. Questa edizione è poi riproposta ed estesa dal 1959 per la madrilena Dólar, mentre infine un'edizione "critica" appare negli anni ' 70 per la Buru Lan. La successione delle pubblicazioni scandisce, in effetti, il varo delle varie leggi franchiste sulla stampa, che riflettono anche i mutati scenari internazionali, e il volume alterna capitoli che descrivono il dettaglio della legislazione con altri che discutono come questa si rifletta sulle differenti edizioni.

Aldilà della coincidenza temporale, però, il personaggio non si piega esattamente alle tesi dell'A. In particolare, Sanchis giudica negativamente la riedizione del 1959, ritenuta sostanzialmente identica alla precedente, mentre le maglie della censura avrebbero in linea di principio potuto permettere una riedizione cronologica delle tavole originali; un fatto probabilmente dovuto più a necessità tecniche di riutilizzo dei cliché - e alla difficoltà di reperire i materiali oltreoceano in anni che ancora non avevano assistito al ritorno di interesse per $\mathrm{i}$ personaggi classici del fumetto - , che a fattori di politica editoriale o culturale. Ciononostante, l'analisi puntuale delle modifiche richieste ed effettivamente implementate negli albi della Hispano 
Americana evidenzia la costante attenzione del regime all'eccessivo erotismo e in generale ai possibili attentati alla morale, lasciando in secondo piano eventuali considerazioni politiche, come testimonia la pubblicazione senza tagli, in piena guerra, dell'avventura gordoniana riguardante la proclamazione della repubblica sul pianeta Mongo. Ancora, le puntuali domande del censore riguardo all'edizione del 1971, sempre disattese (senza alcuna conseguenza pratica) dall'editore, esemplificano il cambio di passo che si stava imponendo nella società civile di quegli anni.

Il volume ha il merito di enucleare quanto di specifico nella legislazione sulla stampa riguardava le pubblicazioni per l'infanzia e la gioventù, e di iniziare una ricostruzione dei criteri e dei meccanismi attraverso i quali si esplicava l'azione della censura franchista su di esse. Ancora, l'A. sottolinea come tale azione abbia influenzato, soprattutto negli anni '50, lo sviluppo di questo settore editoriale, dettando spesso temi e formati: un argomento che elabora ulteriormente nel suo Tebeos mutilados. La censura franquista contra Editorial Bruguera (Ediciones B, Barcelona 2010). Al tempo stesso, Sanchis non tratta puntualmente le fonti, a cominciare da quelle bibliografiche; basando il lavoro su precedenti ricostruzioni, quelle di Martin e di Justino Sinova sulla stampa franchista, per cui non è sempre facile distinguere il suo contributo, tranne che per le puntuali analisi degli interventi censori su Gordon.

Va detto che il volume contiene varie imprecisioni fattuali sulle pubblicazioni italiane anteguerra, dunque su argomenti assodati nella pubblicistica (una ricostruzione puntuale in Ernesto G. Laura, Gli anni de L'Avventuroso, Nerbini, Firenze 1997), anche se non inficiano la ricostruzione generale. Soprattutto, è la correlazione fra interventi della censura fascista e pubblicazioni spagnole che sembra non reggere, non tenendo conto del modo di approvvigionarsi dei materiali originali da parte della Vecchi. La Hispano Americana non utilizzava i canali italiani, né ristampava le pubblicazioni nerbiniane, ma ricorreva direttamente al distributore europeo, la parigina Opera Mundi, e questo rendeva meno significativi nel contesto spagnolo gli effetti sullo stampato della politica censoria fascista contro i comics americani, se non al limite come fonte di ispirazione. È in apparenza un'imprecisione comune ad altre ricostruzioni, e una puntuale descrizione di queste dinamiche è comunque successiva al volume in oggetto (cfr. Fabio Gadducci, Leonardo Gori, Sergio Lama, Eccetto Topolino. Lo scontro culturale tra fascismo e fumetti, Npe, Roma 2011).

Per quel che riguarda gli studi italiani, il volume offre degli interessanti spunti di lavoro. Anzitutto va ricordato che, a differenza che in Spagna, nel nostro paese la censura sulla stampa per ragazzi fu preventiva solo durante la seconda guerra mondiale, quando la produzione straniera era ormai al bando. Nel nostro paese dunque non esistono documenti che testimoniano l'intervento diretto del censore, e ben pochi anche per la produzione autoctona, la maggiore eccezione per il personaggio Dick Fulmine (cfr. Juri Meda, Sbirro e prefetto... Fascista perfetto, «Schizzo», 12, 2002, pp. 5-25). La testimonianza di tale documentazione può quindi aiutarci a intuire le ragioni delle politiche adottate in Italia nel corso degli anni ' 30 , e perfino a meglio inquadrarle nel discorso dello stato totalitario, dato che le prime leggi franchiste sulla stampa richiamano esplicitamente quelle messe in atto solo alcuni anni prima dai regimi fascista e nazista. Anche se, a giudicare dagli interventi imposti sulle avventure di Flash Gordon, la censura spagnola non sembra essere portatrice di alcun elemento xenofobo, caratteristico invece dell'intervento fascista, che arriverà a bandire la stessa forma "fumetto", ovvero l'uso delle nuvolette, come una barbara invenzione americana. 
Soprattutto, questo studio aiuta a gettare nuova luce sulla figura di Lotario Vecchi, pioniere italiano della narrativa popolare, il cui impero editoriale, che si estendeva fino al Sud America, attende ancora di essere esplorato con attenzione (oltre a Eccetto Topolino, esiste solo una monografia scritta da un appassionato: Ezio Ferraro, Lotario Vecchi editore, «Comics», 14, 1974). Dal volume emerge il profilo di una figura in chiaroscuro, che in Spagna agisce in piena conformità alle regole, nonostante la patente di antifascista e il controllo occhiuto al quale lo sottoponeva il regime italiano. Un editore, comunque, che avrebbe continuato a svolgere un ruolo fondamentale per l'esportazione dei fumetti italiani tra gli anni '40 e '50 (da Tex a Pecos Bill, da Pantera Bionda a «Grand Hotel»: cfr. Los tebeos de posguerra, Multiversa, Madrid 2010), e la cui opera ancora oggi attende di essere riscoperta.

Fabio Gadducci

\section{Giorgio Vecchio \\ L'Italia del Vittorioso \\ Ave, Roma 2011,pp. 245, ill.}

Il volume si inserisce a pieno titolo nell'alveo delle recenti ricerche che stanno faticosamente facendo emergere, dal limbo in cui erano relegate, quelle pubblicazioni cattoliche educative per l'infanzia a lungo trascurate dal mondo della ricerca. Vecchio ripercorre le vicende del «Vittorioso» dalla sua fondazione nel 1937, voluta dalla Anonima Veritas Editrice (Ave), al 1966, anno della fusione di questo settimanale col periodico «Vera Vita» che dette origine al «Vitt», una nuova testata che, a dispetto del nome, si discostava alquanto dal «Vittorioso» delle origini.

Il percorso del «Vittorioso», considerato una delle principali imprese culturali popolari del '900, viene incastonato all'interno delle vicende più generali che segnarono un trentennio di storia italiana e del mondo cattolico, e viene analizzato incrociando la lettura del periodico con la documentazione conservata presso l'Archivio dell'Istituto Paolo VI per la storia dell'Azione cattolica e del movimento cattolico e l'Archivio dell'Azione cattolica. Va detto che l'opera si discosta da lavori precedenti anche perché, forse per la prima volta, la storia del settimanale viene strettamente intrecciata con quella dell'Azione cattolica e dell'editrice Ave: sorta ufficialmente nel 1935 con lo scopo di pubblicare libri e periodici ispirati ai princìpi cristiani e distinta dalla già esistente Studium, una casa editrice più legata agli ambienti intellettuali e in particolare alla Fuci, la federazione degli universitari cattolici.

Fu Luigi Gedda, presidente della Gioventù italiana di Azione cattolica (Giac), a promuovere la pubblicazione di un «giornale illustrato per ragazzi», destinato a garantire un'ampia diffusione - a differenza de «L'Aspirante», un periodico dell'Azione cattolica volto essenzialmente alla formazione interna dei suoi membri -, e a contrapporsi alla stampa laica, giudicata responsabile di un pericoloso "slittamento morale" per bambini e adolescenti. Primo direttore responsabile del nuovo foglio e animatore dell'operazione fu don Francesco Regretti; un gran battage pubblicitario e la mobilitazione sia delle sezioni Aspiranti sia dei gruppi di "bonstampisti" (che si occupavano della diffusione delle pubblicazioni cattoliche, la cosiddetta "buona stampa"), accompagnarono in tutta Italia l'uscita del primo numero, il 9 gennaio 1937. La grafica lasciava ancora a desiderare; le storie proposte erano decisamente poco avvincenti; il tono generale della pubblicazione rifletteva chiaramente il clima dell'Italia fascista; tuttavia, il settimanale adottò fin da subito la tecnica del fumetto, sfidando la diffidenza generale, e quella particolare del mondo cattolico, nei confronti di questa modalità espressiva.

«Il Vittorioso» si affidò a disegnatori e narratori italiani, non soltanto per una 
scelta autarchica legata alle direttive del regime, ma anche per una precisa finalità educativa, quella di una formazione integrale della persona attraverso i princìpi e i valori di un cattolicesimo militante. L'A. ricostruisce, infatti, l'affiancarsi e il succedersi di due generazioni di illustratori che traghettarono il periodico dal ventennio fascista all'Italia dell'immediato dopoguerra e della ricostruzione. Il settimanale rappresentò una sorta di palestra per disegnatori e sceneggiatori di fumetti come Sebastiano Craveri, Curt Caesar (Kurt Kaiser), Raffaele Paparella o Gianluigi Bonelli ai quali, nel dopoguerra, si affiancarono artisti come Benito Jacovitti, Franco Caprioli, Gianni De Luca, Ruggero Giovannini, Roudolph (Raul Traverso), Renata Ghelardini De Barba, Lino Landolfi, per citare i più conosciuti. Giustamente, Vecchio concede un particolare rilievo a Jacovitti che, appena diciassettenne, avviò una collaborazione col periodico che presto lo fece diventare, nonostante l'alto livello dei colleghi, il «dominus incontrastato» del giornale. Numero dopo numero Jacovitti affinò la sua matita, fino a giungere alla realizzazione dei suoi tipici paginoni zeppi di figure e situazioni esilaranti, e alla realizzazione di personaggi che riscossero grande successo, da Pippo, Pertica e Palla a Tex Revolver, anticipazione del celebre Cocco Bill.

Nelle annate del «Vittorioso» troviamo storie a sfondo biblico o con un'ambientazione ai tempi delle persecuzioni cristiane, cineromanzi storici, d'avventura o ispirati al neorealismo, racconti umoristici a nuvolette: un insieme di opere che testimoniano la volontà di riempire i fumetti di contenuti formativi adatti al mondo cattolico, così come le varie rubriche che completavano il giornale cercando di aprirsi al dialogo con i giovani lettori. Queste avrebbero acquistato maggiore importanza tra gli anni ' 50 e ' 60 , spaziando dalla filatelia all'angolo della posta e, finalmente, ritagliando anche uno spazio, per quanto margi- nale, alle ragazze. In quegli anni, l'aumento del numero di pagine consentì la pubblicazione di racconti più lunghi, firmati da Piero Bargellini, Giuseppe Fanciulli, Eros Belloni, Vittorio Emanuele Bravetta, Alberto Manzi.

L'attenzione del «Vittorioso» ai diversi sport rifletteva le trasformazioni sociali del paese. Nella seconda metà degli anni ' 40 a farla da padrone era il ciclismo, con il Giro d'Italia seguito tappa dopo tappa e con la "leggendaria" sfida tra Fausto Coppi e Gino Bartali, campione cattolico per eccellenza e "amico" del «Vittorioso». Anche l'alpinismo, considerato dal mondo cattolico un simbolo di elevazione spirituale, trovava ampio spazio fra le pagine del periodico che celebravano gli «eroi della montagna», come Compagnoni, Lacedelli o Bonatti. Il calcio si sarebbe fatto spazio solo a partire dagli anni '50, ma la pubblicazione di copertine con fotografie di squadre della Serie A tra il 1959 e il 1961 preannunciava, secondo l'A., un calo di fantasia e di cura nella fattura del periodico.

Col nuovo decennio si aprì, in effetti, la fase conclusiva del «Vittorioso». La parabola del periodico subì tensioni e vicende che attraversavano tanto l'Ave quanto la Giac, impegnata a distinguersi maggiormente da una casa editrice madre che sembrava ormai agonizzante. Paradossalmente, proprio la ripresa delle attività della casa editrice coincise con la crisi irreversibile del settimanale. Il mondo stava cambiando rapidamente e persino la presidenza della Giac auspicò per «Il Vittorioso» una profonda modifica strutturale «per farne un giornale che sia diretto ai giovani della nuova Scuola dell'obbligo affiancandoli nella loro ascesa culturale». Se, da un lato, la qualità dei fumetti calava, dall'altro si cercò di aprire le pagine del giornale a temi di attualità, dando più spazio ai problemi sociali, alla politica italiana o alle questioni internazionali. Tuttavia, il tentativo di intercettare umori ed esigenze di una generazione di giovani che si appre- 
stava a vivere l'esplosione del ' 68 , portò il periodico a porre in primo piano gli artisti in voga e gli astri nascenti di una televisione che sempre più ipnotizzava $\mathrm{i}$ ragazzi. Il tentativo fallì. Nell'ultimo numero del 1966, il capo redattore Domenico Volpi dette l'addio ai propri lettori. Il primo gennaio 1967 «Il Vittorioso» veniva sostituito dal «Vitt»: un giornale diverso che, come sottolinea l'A., appartiene a un'altra storia.

Integrano efficacemente il volume le riproduzioni di numerose copertine e di otto storie complete, ciascuna appartenente ad autori e a generi differenti; si tratta di una selezione che, dalle prime pagine del 1937 ai primi numeri del «Vitt» di trent'anni dopo, permette al lettore di saggiare concretamente la storia e la parabola del «Vittorioso», offrendo la possibilità di rintracciare visivamente quanto qui argomentato, ma anche di riassaporare vecchie storie o vecchi fumetti che ancora oggi ci divertono e troviamo avvincenti.

\section{Ilaria Mattioni}

David Hajdu

Maledetti fumetti!

Come la grande paura

per i "giornaletti" cambiò

la società statunitense

prefazione di Roberto Giammanco

postfazione di Matteo Sanfilippo

Tunué, Latina 2010, pp. 453

(ed. or. The Ten-Cent Plague:

The Great Comic-Book Scare

and How It Changed America, Farrar,

Straus and Giroux, New York 2008)

In molti paesi del mondo occidentale, ma non solo, tra gli anni ' 40 e '50, davanti al proliferare di pubblicazioni a fumetti di diverso genere (bellici, western, fantascientifici, polizieschi, horror), lette soprattutto da un pubblico di bambini e adolescenti, ci fu una vera e propria caccia alle streghe volta a limitarne la circolazione. Allarmanti furono le prese di posizione di psicologi, educatori e peda- gogisti, numerose le iniziative giuridiche e politiche, le proposte di legge per arginare quello che veniva visto come un virus che metteva in pericolo la salute mentale di bambini e adolescenti.

I fumetti erano considerati i diretti responsabili della crescente delinquenza minorile, della sessualità sfrenata e del sadismo dei lettori, indotti a comportamenti violenti per imitare le gesta dei loro eroi di carta. Accusati peraltro di propaganda comunista o diffusione di valori fascisti, i fumetti erano considerati nemici della lettura, in quanto la presenza delle immagini e di brevi dialoghi - concitati, spesso sgrammaticati e ortograficamente errati - distoglieva i ragazzi dalle "buone letture", con gravi ricadute per un "buon apprendimento".

Tale ondata antifumettistica e moralizzatrice - che favorì in molti casi il varo di codici e leggi in difesa della gioventù dalla cattiva stampa - interessò paesi come l'Italia (cfr. Juri Meda, Stelle e Strips. La stampa a fumetti italiana tra americanismo e antiamericanismo (1935-1955), Eum, Macerata 2007), le due Germanie (Stephan Buchloh, Pervers, jugendgefährdend, staatsfeindlich. Zensur in der Ära Adenauer als Spiegel des gesellschaftlichen Klimas, Campus-Verl, Frankfurt am Main 2002; Gerd Lettkemann-Michael F. Scholz, Schuldig ist schließlich jeder... Der Comics besitzt verbreitet oder nicht einziehen, Steinchen für Steinchen Verlag, Berlin 1994), la Francia (Richard I. Jobs, Tarzan under Attack: Youth, Comics and Cultural Reconstruction in Postwar France, «French Historical Studies», 4, 2003, pp. 687725), l'Austria (Matthew Paul Berg, Schmutz, Schund and Sittliche Gefährdung: The Politics of Children's Morality Legislation in Early Postwar Austria, "German Studies Review», 1998, n. 3, pp. 447-67) nonché gli Stati Uniti.

Negli Usa, alla metà degli anni '40, gli albi a fumetti vendevano ogni settimana tra gli 80 e i 100 milioni di copie; albi che poi magari passavano per le ma- 
ni di amici dell'acquirente, spingendo verso l'alto il numero di persone che entravano in contatto con questi prodotti. Alimentati dagli stessi temi di cui si erano nutriti e continuavano a nutrirsi romanzo popolare e cinema noir, molti dei successi di mercato di fine anni '40 e dei primi anni ' 50 narravano storie dell'orrore, di crimini in ambienti dove regnava il vizio e la lussuria. Per un certo periodo, la pubblicazione di questi nuovi giornaletti crebbe in maniera incontrollata, senza quelle regolamentazioni che invece riguardavano, ad esempio, cinema, radio o televisione.

Tali letture - giudicate spudorate, disinibite, romantiche quanto criminose costituirono il terreno di scontro e di campagne proibizioniste e morali. Comunità di cittadini e parrocchie organizzarono iniziative anti fumetto inscenando processi contro personaggi dei comics; in alcune scuole $\mathrm{i}$ fumetti furono persino messi al rogo. Numerose iniziative legislative a livello statale o municipale bandirono o limitarono la vendita di questi giornaletti, portando quasi all'annientamento dell'industria del comic book.

$\mathrm{Va}$ ricordato che i fumetti moderni, sin dalla loro prima apparizione sui supplementi domenicali dei quotidiani statunitensi a fine ' 800 , erano stati etichettati - per usare le parole di Ralph Bergengren pubblicate sull' «Atlantic Monthly» nell'agosto 1906 - come letture «per persone estremamente stupide», «una vicenda di vergogna e degrado per la nazione». Nel 1940, il noto scrittore per ragazzi Sterling North sul «Daily News» aveva definito l'editoria a fumetti come un «fungo velenoso». Nel 1954, lo psichiatra di origine tedesca Fredric Vertham, nel suo trattato che divenne un manifesto per la campagna antifumettistica dell'epoca, Seduction of the innocent. The influence of comic books on today's youth, sostenne che i fumetti erano dannosi soprattutto per i più giovani e che costituivano la causa primaria della delinquenza minorile. «I think Hitler was a beginner compared to the comic-book industry», disse Wertham nell'aprile del 1954, durante un'audizione, trasmessa anche in Tv, organizzata dalla Sottocommissione senatoriale per lo studio e l'analisi della criminalità giovanile.

Questa campagna moralizzatrice travolse nei primi anni '50 il fumetto Usa; lasciò in vita solo 250 delle 650 testate già attive e fu cavalcata da politici, anche a fini elettorali, come il senatore democratico del Tennessee Estes Kefauver, e il senatore repubblicano del New Jersey Robert C. Hendrickson.

La vicenda è raccontata, più che ricostruita, in questo volume di stile giornalistico; un lavoro basato soprattutto su 150 testimonianze di disegnatori, editori, autori e redattori, soprattutto di area newyorkese, colpiti direttamente o interessati dalla campagna antifumettistica che coincise cronologicamente con la seconda Red Scare fomentata dal senatore Joseph McCarthy, con il risultato che gli autori di fumetti furono spesso additati come persone sgradite al pari dei comunisti. Le interviste, come sottolinea Matteo Sanfilippo nella postfazione, restituiscono anche la storia di una porzione di un mondo editoriale, spesso dimenticato dalla storia dell'editoria, e di un vasto proletariato della carta stampata composto da disegnatori, il più delle volte giovanissimi, desiderosi di creare storie e personaggi sempre nuovi.

Il volume è quindi soprattutto un corposo, e talvolta eccessivamente ricco di dettagli sulle biografie e le tecniche degli autori, repertorio documentario che l'A. usa giornalisticamente additando i "cattivi" di questa caccia alle streghe, Wertham in primis, non tenendo conto di altri protagonisti di quella campagna moralizzatrice, come i quotidiani e i periodici che pubblicavano anch'essi comics e che volevano quindi togliere dal mercato la concorrenza degli albi a fumetti, né talvolta cogliendo le sfumature nelle posizioni antifumettistiche più ragionate $\mathrm{e}$ meno scandalistiche, come quelle avan- 
zate nel 1945 dallo studioso Walter J. Ong nelle aule universitarie.

Oltre alle testimonianze, Hajdu cita articoli pubblicati su «New York Times», «Chicago Daily Tribune», «The News», «Los Angeles Times», «The Washington Post», integrandoli con notizie tratte da fonti istituzionali, citate però nelle note senza precisi riferimenti archivistici, salvo generiche indicazioni che richiamano 1'Archivio Robert C. Hendrickson presso la Bird Library della Syracuse University, i documenti riguardanti la Senate Subcommittee on Juvenile Delinquency presieduta da Hendrickson e la Senate Special Committee to Investigate Organized Crime in Interstate Commerce presieduta da Kefauver, conservati presso i National Archives di Washington, indicati nelle note solo come «archivi nazionali», rendendo quindi non immediatamente identificabile la collocazione archivistica.

Va però sottolineato, per l'edizione italiana, l'utile intervento editoriale e metodologico del curatore Marco Pellitteri, che ha aggiunto informazioni sugli autori citati, integrando dati e riferimenti bibliografici mancanti nell'edizione originale per deliberata scelta dell'autore. Date queste carenze di ordine metodologico, il volume costituisce quindi non un punto di arrivo ma di partenza per una ricerca, magari transnazionale, su una campagna censoria non estranea a interessi di tipo politico nel contesto della guerra fredda.

Nicola Spagnolli

Isabelle Doré-Rivé-Guy Krivopissko

(sous la direction de)

Traits résistants.

La Résistance dans la bande

dessinée de 1944 à nos jours

Libel, Lyon 2011, pp. 183, ill.

Il volume, pubblicato in occasione della mostra omonima organizzata nel 2011 presso il Centre d'Histoire de la Résistance et de la Déportation de Lyon, in collaborazione col Musée de la Resistance nationale di Champigny-sur-Marne, raccoglie contributi di storici, giornalisti e bibliotecari che con approcci diversi concorrono ad analizzare le rappresentazioni della Resistenza francese nei fumetti, dagli anni '40 ai giorni nostri.

Riccamente illustrata, arricchita da una lista delle fonti e da una sintetica bibliografia su Bande dessinée $(B D)$ e Resistenza, l'opera si presenta con un carattere ibrido, alternando saggi che analizzano puntualmente singole opere o collane, con articoli di taglio divulgativo, contributi concepiti per archivisti o appassionati della materia, riproduzioni di opere inedite, interviste. L'assenza di una solida introduzione non aiuta a dare rilievo ai temi, ai problemi, alle chiavi interpretative che questo lavoro collettaneo intende affrontare; purtroppo, la pur puntuale premessa dei curatori (pp. 4-5), e soprattutto il rapido intervento introduttivo di Laurent Douzou, contemporaneista dell'Institut d'études politiques de Lyon (La Résistance, une histoire dont on peut rendre compte?, pp. 7-10), non sembrano colmare questa lacuna. In compenso, il contributo dell'archivista Xavier Aumage (Il était une fois la Résistance dans la bande dessinée, pp. 1257), commissario della mostra, offre un quadro generale per ricostruire l'evoluzione delle rappresentazioni della Resistenza nella $B D$, e delle diverse tipologie di fumetti, dagli anni della guerra in poi.

L'analisi è di tipo cronologico e tematico; prende le mosse dal periodo dell'occupazione quando, tra 1940 e 1944, sia la propaganda vichysta sia quella tedesca usarono i fumetti più popolari, o ne crearono di inediti, per consolidare il nuovo sistema di potere, presentare in modo idilliaco il Service du travail obligatoire e screditare l'immagine dei resistenti, disegnati come banditi sanguinari assoldati da stranieri, ebrei, comunisti. Se è vero che anche la forzatamente ben più fragile stampa clandestina usava i fumetti come arma di propa- 
ganda e denuncia, lo squilibrio dei mezzi a disposizione per le diverse forze che si fronteggiavano costringe, oggi, gli studiosi a ricercare con fatica negli archivi le poche tracce giunte fino a noi di questa produzione di contrabbando, volta a ribaltare la propaganda di regime e a sostenere il maquis, all'epoca ancora presentato come un luogo distante e misterioso.

Fu piuttosto tra la Liberazione e il 1947 che opere a fumetti, poi divenute celebri, concorsero a rendere popolare il termine maquis (p. 25). In quella fase la $B D$, spesso riproducendo modelli di narrazione già consolidati, contribuì - spiega Aumage - a cristallizzare un archetipo del resistente destinato a durare a lungo, presentato come espressione migliore di una Francia priva di fratture interne, unita nella lotta contro l'oppressione tedesca; un eroe collocato nella linea di discendenza del pantheon dei grandi uomini della storia nazionale, talvolta rappresentato da figure come il colonnello Fabien, il generale Leclerc, de Gaulle o Guy Môquet (pp. 18, 167). È quindi nei fumetti di metà anni ' 40 che sono individuati i temi più ricorrenti nella produzione di $B D$ sulla Resistenza francese: da quello dell'unità nazionale forgiata nella lotta, e che sovrasta ogni differenza interna all'Esagono, al tema della violenza e della lotta armata, le cui rappresentazioni nei fumetti furono costrette a modificarsi in modo significativo, o a sparire del tutto, col varo della legge del 16 luglio 1949 sul controllo e la censura delle pubblicazioni destinate ai giovani.

Solo in una fase successiva, e col rinnovamento che attraversò pure il mondo dei fumetti nei primi anni ' 70 , nella $B D$ sulla Resistenza si sarebbero iniziati ad affrontare anche temi più difficili e impegnativi, come la deportazione e il collaborazionismo, le fratture interne al fronte antifascista, le relazioni di genere. Il dato, in qualche modo, trova conferma anche negli altri contributi del volume. Penso, ad esempio, all'intervento di $\mathrm{Au}$ - mage sui fumetti più recenti (Concevoir une bande dessinée sur la Résistance); all'intervista rilasciata da Stéphane Lavallois (autore di un'opera che intreccia memorie di famiglia e contesto storico: La résistance du sanglier, Futuropolis 2008); ai due articoli di Sylvain Lasage: il primo dedicato al ruolo degli editori francesi e belgi, e agli elementi di continuità e di frattura del mercato editoriale tra il periodo dell'occupazione e il dopoguerra, l'altro centrato sull'analisi delle diverse tipologie di pubblicazione e sulle conseguenze, di lunga durata, della legge del 1949.

Forse, il contributo più interessante è offerto da Jean-Pierre Mercier, interamente dedicato a La bête est morte! La guerre mondiale chez les animaux, uno splendido fumetto a colori pubblicato in due fascicoli di grande formato nel 1944-1945 e, a seguito dell'immediato successo editoriale, riedito più volte fino ai giorni nostri. Disegnato con maestria da Edmond-François Calvo su testi di Victor Dancette, concepito alla vigilia della Liberazione, il fumetto - riprendendo tradizioni consolidate - trasferisce in un universo di animali la guerra con, ad esempio, i tedeschi rappresentati da lupi, gli italiani da iene, i giapponesi da scimmie, gli inglesi da bulldog, i sovietici da orsi, i partigiani da cicogne e così a seguire. Col suo dispositivo narrativo canonico (la storia si apre col racconto di un nonno reduce e mutilato di guerra ai nipotini), i suoi silenzi (solo in due piccole vignette si fa allusione alla sorte degli ebrei, ed è quasi assente ogni riferimento a Vichy o alla collaborazione) e la sua retorica (esercito forte e nazione unita sono «la certitude de la Grandeur»), l'opera è considerata uno dei maggiori e più longevi esempi di propaganda della Resistenza francese. Assai interessanti sono le informazioni fornite da Mercier riguardo alle biografie degli autori, e in particolare su quella di Dancette che, col suo «fervore patriottico e gaullista», all'uscita della guerra cercava di cancel- 
lare il suo recente passato di autore di testi petainisti su libri illustrati, volti a esaltare l'ardore della «splendida gioventù» arruolata nella milizia e pubblicati da Éditions G.P., la stessa casa editrice de La bête est morte! (p. 63).

La riproduzione, alle pp. 162-65 del volume, dell'inedita biografia di Oberdan Chiesa, antifascista toscano ucciso nel 1944 , realizzata una trentina di anni fa dal milanese Guido Crepax e ora conservata nel Musée de la Résistance nationale (L'attentato, 4 tav.), se ha poco a che fare con un catalogo dedicato ai fumetti francesi, dà modo al lettore di capire rapidamente quanto si siano modificati i temi e gli stili dei fumetti nel corso della seconda metà del ' 900 , non raramente in sintonia con l'evoluzione del dibattito storiografico.

Roberto Bianchi

René Mounajed

Geschichte in Sequenzen.

Über den Einsatz

von Geschichtscomics

im Geschichtsunterricht

Peter Lang, Frankfurt am Main 2009, pp. 301

Dietrich Grünewald (Hrsg.)

Struktur und Geschichte

der Comics.

Beiträge zur Comicforschung

Christian A. Bachmann Verlag,

Bochum und Esse 2010, pp. 326

La Gesellschaft für Comicforschung (Gfcf), costituitasi in associazione nel 2005, ha come principale interesse quello di occuparsi di fumetto, soprattutto dell'aera tedesca, con metodo scientifico attraverso un approccio multidisciplinare. Presieduta da Dietrich Grünewald, docente presso l'Institut für Kunstwissenschaft dell'Università di CoblenzaLandau, la Gfcf conta tra i propri membri accademici di diversi ambiti, come Bernd Dolle Weinkauff, docente di storia del fumetto alla Goethe Universität di
Francoforte nonché responsabile dell'archivio dell'Institut für Jugendbuchforschung, Michael F. Scholz, docente di storia moderna alla Gotland University e René Mounajed, insegnante e già collaboratore dell'Università di Göttingen per lo studio della didattica della storia. Dal 2005 la Gfcf edita i Deutsche Comicforschung (pubblicati a Hildesheim dalla Sackmann und Hörndl), annuari che raccolgono contributi, corredati di apparati iconografici, riguardanti la storia del fumetto, soprattutto tedesco, sotto diverse prospettive: storia della stampa periodica, della cultura di massa e dei media, della propaganda, dell'illustrazione e della semiotica del fumetto. La Gfcf ha poi organizzato presso Coblenza tre convegni riguardanti la struttura e la storia del fumetto in Germania, la ricerca sul fumetto nell'ambito dei cultural studies, come campo interdisciplinare e come oggetto di ricerca sociale.

Il volume Struktur und Geschichte der Comics, curato da Grünewald, raccoglie una selezione di interventi presentati ai tre convegni. Il risultato è un'eterogenea quanto vasta indagine sullo stato dell'arte della ricerca tedesca sui diversi aspetti di questo media, con contributi di buon livello anche se non sempre innovativi rispetto a quanto già pubblicato sull'argomento. E questo il caso dei saggi di Grünewald (pp. 11-31), di Marianne Krichel (pp. 33-46) e Stephen Packard (pp. 47-65), i quali svolgono delle analisi di ordine storico e semiotico sul linguaggio del fumetto rifacendosi soprattutto ai contributi di Will Eisner (1917-2005; autore che ha definito la narrazione nei comics come «arte sequenziale», che procede mediante sequenze narrative costituite da immagini e testi strettamente collegati tra di loro: Comic and Sequential Art, Poor House, Guerneville 1985), e di Scott McCloud, autore di un saggio che analizza a fumetti il linguaggio dei comics (Understanding Comics, Tundra Publishing, Northampton 1993).

I contributi più interessanti e innovativi si occupano però della narrazione sto- 
rica attraverso il fumetto e del fumetto come fonte per la ricerca storica. Oliver Näpel (pp. 99-127), utilizzando un modello teorico di analisi dei meccanismi di costruzione dell'immagine del diverso, compie un rapido, e superficiale, excursus storico della dicotomia identità/alterità realizzata iconograficamente attraverso la stereotipizzazione del diverso, l'utilizzo della (ri)costruzione storica e delle rappresentazioni mediali della storia, dall'antica pittura vascolare greca sino ai fumetti e ai film. Hubert Mittler (pp. 155-77) analizza l'immagine del medioevo e i suoi stereotipi, ad esempio la figura del cavaliere, nelle serie a fumetti Prince Valiant dell'americano Harold Foster, pubblicata anche in Germania - per la prima volta nel 1939 e successivamente nel 1950 - e in quella del belga Hermann Huppen Les Tours de Bois-Maury (1984-1994), pubblicata anche in Germania con il titolo Die Türme von Bos-Maury (Carlsen, Amburgo 1986-1994).

Se Detlev Gohrbandt (pp. 179-98) ricostruisce la storia delle versioni inglesi del fortunato libro illustrato per ragazzi Struwwelpeter (1845) dello scrittore tedesco Heinrich Hoffmann, e in particolare delle versioni satiriche pubblicate durante le due guerre mondiali in funzione rispettivamente antitedesca e antinazista, Michael F. Scholz (pp. 199-217) ripropone la sua tesi del fumetto come fonte per la ricerca storica, già abbozzata molti anni fa (Comics: eine neue historische Quelle?, «Zeitschrift für Geschichtswissenschaft», 11, 1990, pp. 1004-10), secondo la quale i comics rappresentano, per diffusione e ruolo nel sistema dei media e nella vita quotidiana, una fonte non solo per la storia dell'iconografia ma anche per lo studio della cultura di massa; una fonte imprescindibile per la storia culturale e sociale del '900. La tesi è sostenuta attraverso un'analisi del caso della Repubblica Democratica Tedesca, dove le riviste a fumetti e la stampa periodica furono utilizzate dalla direzione della Sed e dalle organizzazioni giovanili (Freje Deutsche Jugend, Junge Pioniere) per costruire una pedagogia socialista attraverso racconti, storie a fumetti o illustrate, celebrative del socialismo, della lotta antinazista da parte dell'Armata rossa o dei risultati raggiunti dall'Urss in campo aerospaziale con il lancio dello Sputnik.

Ralf Palandt (pp. 219-55) ricostruisce invece il difficile percorso, tra censure e modifiche, del racconto del nazionalsocialismo attraverso il fumetto nella Repubblica federale tedesca. Difficoltà dovuta soprattutto alle leggi, a partire da quella sulla diffusione di testi pericolosi per la gioventù del 1953 - sul cui rispetto vigilava un'apposita Commissione federale istituita l'anno successivo, o il codice penale tedesco -, che vietavano la riproduzione della svastica e di altri simboli nazisti all'interno di pubblicazioni o rappresentazioni. Il caso più emblematico di questa legislazione, volta a reprimere qualsiasi rigurgito nazionalsocialista, fu il processo per propaganda nazionalsocialista svoltosi a Sonneberg nel 1995 nei confronti di due editori tedeschi che avevano inserito una svastica sul manifesto pubblicitario della traduzione tedesca del libro Maus, un'opera a fumetti scritta dall'americano Art Spiegelman dove l'A. racconta la vita del padre, polacco di religione ebraica deportato ad Auschwitz, mettendo in scena il processo di redazione della storia attraverso espedienti narrativi originali e atipici che aprirono al fumetto spazi fino ad allora inesplorati.

René Mounajed (pp. 129-54) affronta un tema più ampiamente sviluppato in Geschichte in Sequenzen. Über den Einsatz von Geschichtscomics im Geschichtsunterricht, ovvero il fumetto storico, le sue diverse declinazioni e il suo potenziale dal punto di vista della didattica della storia. Come qualsiasi altro linguaggio narrativo, infatti, anche il fumetto si è misurato con il racconto storico con diversi sottogeneri qui individuati. In 
quello definito Geschichts-Fantasiecomics il contesto storico viene usato solo come sfondo per ambientare narrazioni favolistiche prive di verosimiglianza. Altre volte (Geschichts-Sachcomics) il fumetto ha il fine di esporre e raccontare fatti, eventi e personaggi storici nella maniera più oggettiva possibile, al limite del didascalico. Inoltre, e siamo alla categoria Geschichts-Romancomics, il fumetto si è occupato di storia costruendo narrazioni fittizie basate però su solidi, documentati e talvolta dichiarati riferimenti storici. Il fumetto a sfondo storico, infine, è servito come strumento di propaganda e indottrinamento politico $(\mathrm{Ge}-$ schichts-Propagandacomics).

Nel quinto capitolo di Geschichte in Sequenzen l'A., rifacendosi in particolare agli studi di Michael Sauer, docente di didattica della storia all'Università di Göttingen, approfondisce la tesi circa il potenziale didattico del fumetto per l'apprendimento della storia, potenzialità limitata solo al Geschichts-Romancomics e al Geschichts-Sachcomics, soprattutto dal punto di vista dello sviluppo delle competenze riguardanti la scienza storica, l'appropriazione di concetti appartenenti alla disciplina, la capacità di orientarsi nella storia, la competenza narrativa (intesa come efficacia nel costruire una narrazione storica fondata), la capacità di saper decostruire un racconto storico contestualizzandolo, analizzando i caratteri dell'autore e le finalità del testo, la filosofia della storia trasmessa o adottata, nonché la competenza nel settore dei media, intesa come acquisizione di un vocabolario specifico, di una capacità interpretativa del funzionamento e del linguaggio del fumetto.

Rispetto ad altre opere sul tema penso ai lavori di Gerald Munier (Geschichte im Comic. Aufklärung durch Fiktion? Über Möglichkeiten und Grenzen des historisierenden Autorencomics der Gegenwart, Unser Verlag, Hannover 2000) e Christine Gundermann (Jenseits von Asterix. Comics im Geschichtsunter- richt, Wochenschau Verlag, Schwalbach/Ts 2007) -, questo volume ha il pregio di non fermarsi all'aspetto teorico, presentando (cap. VI) indagini sul campo svolte o da altri studiosi o condotte dall'A. in Germania, ad esempio su alcune ottave classi del ginnasio (alunni di 14 anni) o su insegnanti di vari istituti scolastici. In questo modo, si fornisce un inedito quadro sulla ricezione del fumetto presso i giovani lettori e sul punto di vista degli insegnanti riguardo al possibile uso del fumetto nella didattica della storia. Dall'indagine condotta dall'A. su 290 docenti risulta infatti, anche piuttosto prevedibilmente, che gli insegnanti di età compresa tra i 20-30 anni, diversamente dai colleghi dai 51 anni in poi, apprezzano i fumetti e considerano il loro utilizzo nella didattica della storia positivo dal punto di vista della motivazione degli studenti alla collaborazione in classe, dell'aumento di conoscenza della storia nonché dello sviluppo di competenze specifiche. Rimane però il problema, osserva l'A. che in appendice offre una ricca bibliografia di fumetti a sfondo storico di diversi paesi divisi per epoca d'ambientazione - preistoria, età del bronzo e del ferro, storia antica, storia medievale, età moderna ed età contemporanea per un totale di 350 titoli - e catalogati per sottogeneri, che i docenti, a parte Asterix e Maus, spesso non conoscono il mondo del fumetto.

La proposta dell'A., pertanto, può essere considerata una buona traccia di lavoro per gli insegnanti di storia che vogliono intraprendere questa strada. Proposta che, però, avrebbe richiesto ulteriori indicazioni metodologiche qui non fornite per quanto riguarda, ad esempio, l'attività didattica di decostruzione di un fumetto storico in modo da insegnare agli studenti come individuare e analizzare le intenzioni dell' autore, dell'editore nonché i riferimenti iconografici e bibliografici utilizzati.

Nicola Spagnolli 
Simona Bassano di Tufillo

Piccola storia dei Peanuts.

Le strisce più famose d'America

tra arte, cultura e linguaggio

Donzelli, Roma 2010, pp. 201

Quando il 2 ottobre 1950 comparvero per la prima volta sulla stampa quotidiana statunitense le strisce di un nuovo fumetto chiamato Peanuts, pochi potevano prevedere lo sfolgorante successo che avrebbero avuto nell'immaginario collettivo. Basti pensare a come la coperta di Linus sia diventata un'espressione usata nel mondo odierno entrando persino nel linguaggio specializzato della psichiatria. È indubbio che tale successo sia in gran parte merito dell'inventore dei Peanuts, Charles Monroe Schulz, rimasto sempre l'unico autore delle vignette fino alla sua scomparsa nel 2000.

Il libro di Simona Bassano di Tufillo, dichiaratamente appassionata di Charlie Brown, Snoopy, Lucy e degli altri protagonisti del fumetto, cerca non tanto di ripercorrere i cinquant'anni di strisce quotidiane dei Peanuts, quanto di scavare tra le tante storie disegnate da Schulz per riuscire a coglierne le grandi capacità espressive. La storia e le sue rappresentazioni restano quindi sullo sfondo di un lavoro che mira a dare dignità artistica all'opera di Schulz - letta sulla base di teorie estetiche, filosofiche e semiologiche -, ma che può risultare utile anche per ricostruire un tassello significativo di storia culturale del secondo '900, oltre che di storia dei fumetti.

L'A., discostandosi dalle teorie di Umberto Eco - che definì i Peanuts dei «piccoli mostri», resi tali dall'introspezione delle brutture del mondo adulto e usando gli strumenti della semiotica, ricorda che uno dei motivi che forse spiega il successo di questi fumetti risiede nel meccanismo di immedesimazione profonda che può scattare in ogni lettore e lettrice verso i principali personaggi delle strisce: Charlie Brown con la sua sfortuna, Linus e le sue nevrosi, Snoopy con la sua fantasia sfrenata, Lucy e il suo pessimo carattere. Si tratta di pregi e difetti che albergano dentro ognuno di noi - spiega l'A. -, e che le strisce di Schulz ci fanno rivivere, non come rapporto metaforico tra l'infanzia e l'età adulta, ma come relazione tra mondo e individuo, utilizzando i protagonisti dei Peanuts e le loro riflessioni sull'esistenza, sul significato della vita, sul rapporto tra l'ambiente circostante e le nostre sensibilità.

È così che si spiegano i dialoghi con le foglie o con i fiocchi di neve che caratterizzano le vignette di Schulz. La natura assume un significato esemplare perché rappresenta una serie di fenomeni sui quali l'uomo si è sempre interrogato. I Peanuts sfruttano gli eventi naturali per porsi domande più o meno metafisiche sul mondo e sull'interdipendenza che esiste tra l'essere umano e l'ambiente. Il fatto che tutto ciò avvenga per bocca di personaggi bambini, seppur originali rispetto ai loro coetanei, rende queste vignette piccoli capolavori, spesso capaci di provocare effetti spiazzanti nei lettori. Non sempre, infatti, le riflessioni di Lucy o la passione di Schroeder per Beethoven risultano di immediata comprensione.

Questo, però, non deve portarci a pensare che i personaggi che Schulz presenta posseggano caratteristiche tanto anomale da renderli assolutamente irreali. Se prendiamo come esempio il cane Snoopy, infatti, non c'è quel processo antropomorfico tipico dei prodotti Disney, nei cui fumetti gli animali perdono le loro caratteristiche precipue e incorporano desideri e aspirazioni umane. Snoopy può sognare di essere l'asso del volo della prima guerra mondiale in lotta contro il "Barone Rosso", usa la macchina per scrivere, filosofeggia sul ruolo della "razza canina" nella società ma, alla fine, resta sempre un cane che litiga con il gatto dei vicini e il cui momento clou della giornata rimane l'ora di cena; dorme in una cuccia, ha una ciotola, gioca con il proprio padrone come tutti i cani del mondo. 
È in questa apparente semplicità che l'A. individua il successo dei Peanuts, che nel tempo ha portato quasi a una serializzazione di alcuni avvenimenti del fumetto, arrivando a comporre un universo proprio al cui interno convivono oggetti pensanti come la scuola frequentata dalla piccola Sally, la sorellina di Charlie Brown, o l'albero mangia aquiloni: elementi che solo apparentemente potrebbero far sembrare quest'opera come destinata ai bambini. Attraverso azioni reiterate nel corso del tempo, in realtà Schulz cerca di far emergere alcune peculiarità del suo lavoro e dei suoi personaggi. La sfortuna di Charlie Brown, ad esempio, non deve portarci alla commiserazione del bambino, incapace di vincere anche una sola partita di baseball, bensì tratteggia l'ostinazione e la fiducia negli altri che il personaggio incarna. La stessa Lucy, sempre scorbutica, appare un personaggio positivo - spiega l'A. anche quando si diletta nel far cadere Charlie Brown a terra, spostando col ditino il pallone da football all'ultimo momento prima del calcio da fermo tentato dal bambino con la testa tonda (altra scena reiterata ogni autunno).

Il fumetto di Schulz è principalmente famoso e apprezzato non per i disegni ipercinetici cui gran parte dei fumetti statunitensi ci ha abituato, né per le scene di azione. L'eterna lotta per il possesso della famosa coperta tra Linus e Snoopy, o l'immancabile partita di baseball persa da Charlie Brown, svolgono un ruolo di cornice all'elemento caratteristico che regge i Peanuts: i dialoghi. La capacità espressiva dei disegni, spesso minimali, infatti, si sposa perfettamente con quelle conversazioni brevi e graffianti che fanno emergere l'individualismo come uno dei tratti tipici della società statunitense secondo Schulz. Ma non si tratta di un individualismo composto di misantropia, opulenza, benessere e indifferenza verso il mondo circostante, bensì di un elemento che domina il piccolo universo al cui interno tendiamo a rinchiuderci, con le nostre piccole ossessioni, cercando comunque di leggere e magari criticare il mondo esterno. Nelle vignette dei Peanuts incontriamo, più che la descrizione di singoli eventi, brevi monologhi che offrono visioni e interpretazioni del reale. Piccoli mondi chiusi presentati attraverso un ricco cast di bambini e animali dai tratti originali che riescono, in poche vignette, a strapparci qualche sorriso e, più profondamente, a donarci punti di vista originali, capaci di innescare riflessioni sui più svariati argomenti. Questo è tanto più vero se guardiamo le vignette di Schulz come tanti piccoli fotogrammi di un lungometraggio, dove ogni scena rappresenta un punto di vista dell'autore esternato attraverso le sue creature di carta. È un aspetto su cui insiste l'A., invitando a leggere le tante storie dei Peanuts come un corpus omogeneo, per quanto modificato nel corso del tempo, anche in modo rilevante: dal tratto, ai dialoghi, ai temi affrontati.

Cinquant'anni di Peanuts hanno accompagnato gli Usa dalla presidenza Eisenhower a quella di Bush jr., riuscendo a mantenere freschezza e acume. Schulz è morto e non ha concesso a nessun altro il diritto di poter disegnare e raccontare le storie delle sue creature. Un "egoismo" che ci ricorda quanto il connubio tra l'autore e l'opera sia stato intenso e completo. Intenso come può oggi esserlo una lettura completa delle sue tante vignette: strumento utile per capire aspetti non secondari di un pezzo di '900 segnato da trasformazioni sociali e politiche profonde.

Antonio Lenzi 
Copyright $(\odot$ FrancoAngeli

N.B: Copia ad uso personale. È vietata la riproduzione (totale o parziale) dell'opera con qualsiasi mezzo effettuata e la sua messa a disposizione di terzi, sia in forma gratuita sia a pagamento. 
Hanno collaborato a questo numero:

\author{
Aldo Agosti \\ Dipartimento di studi storici, Torino \\ aldo.agosti@yahoo.it
}

\section{Eszter Bartha}

Department of Eastern European History, Eötvös Loránd University, Budapest barthaeszter@hotmail.com

\section{Roberto Bianchi}

Dipartimento di storia, archeologia, geografia, arti e spettacolo, Firenze roberto.bianchi@unifi.it

\section{Marco Bresciani}

Dipartimento di civiltà e forme del sapere, Pisa

brescia77@yahoo.com

\section{Gennaro Carotenuto}

Dipartimento di studi umanistici,

Macerata

gcarotenuto@unimc.it

\section{Marcel Farinelli}

Departamento de humanidades, Universitat Pompeu Fabra, Barcelona marcel.farinelli@upf.edu

\section{Eric Foner}

History Department, Columbia

University, NYC

ef17@columbia.edu

Paolo Fonzi

Dipartimento di lettere e beni culturali, Napoli 2, S.M. Capua Vetere (CE) casafon@hotmail.com

Daria Frezza

Università di Siena

bicocchi.clem@dada.it

Fabio Gadducci

Dipartimento di informatica, Pisa gadducci@cli.di.unipi.it

\section{Ulrich Herbert}

Albert-Ludwigs-Universität, Friburg ulrich.herbert@geschichte.uni-freiburg.de

\section{Fabiana Loparco}

Dottorato di ricerca in History of Education, Macerata fabianaloparco@libero.it

Antonio Lenzi

Dottore di ricerca in Storia dei partiti e dei movimenti politici, Urbino antoniolenzi@hotmail.it

Alessandra Lorini

Università di Firenze

loriniflorence@yahoo.it

\section{Ilaria Mattioni}

Università cattolica S. Cuore, Milano

Ilaria.Mattioni@unicatt.it

\section{Juri Meda}

Dipartimento di scienze della formazione, dei beni culturali e del turismo, Macerata juri.meda@unimc.it

Hans Mommsen

Ruhr-Universität, Bochum

Hans.Mommsen@t-online.de

\section{Marcella Simoni}

Dipartimento di studi sull'Asia e sull'Africa mediterranea, Venezia msimoni@unive.it

Nicola Spagnolli

Dottore di ricerca in Storia contemporanea, Verona nicola.spagnolli@gmail.com

Michael Wildt

Humboldt Universität, Institut für Geschichtswissenschaften, Berlin michael.wildt@geschichte.hu-berlin.de 


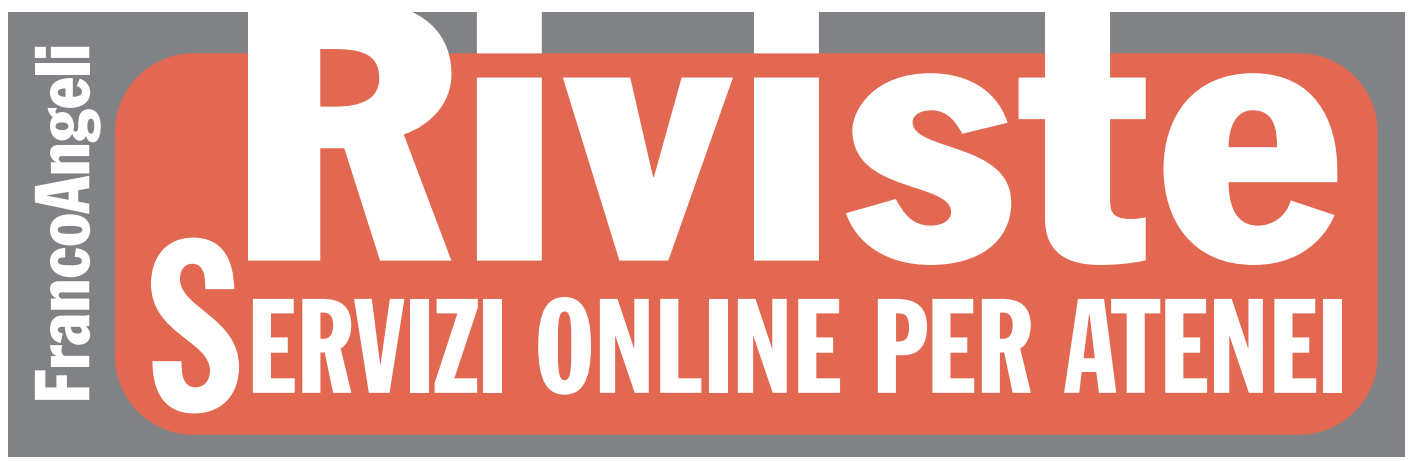

Dal 2013 oltre 80 riviste FrancoAngeli sottoscrivibili per gli Atenei in versione online con diritto d'accesso perpetuo al corrente e a tutti gli arretrati disponibili.

Tramite un'unica licenza è possibile accedere:

- alle versioni in formato digitale delle annate in corso;

- a tutte le annate arretrate (a partire dal 2000, se disponibili) in formato digitale;

- con diritto d'accesso perpetuo.

Le modalità di accesso consentono:

- la ricerca (per autore, per titolo, full-text);

- la visualizzazione del documento (corrispondente a quello dell'edizione cartacea).

Come facoltà opzionali sono previsti:

- diritti di accesso da remoto tramite proxy server;

- diritti di effettuare copia e incolla (download) ad uso personale.

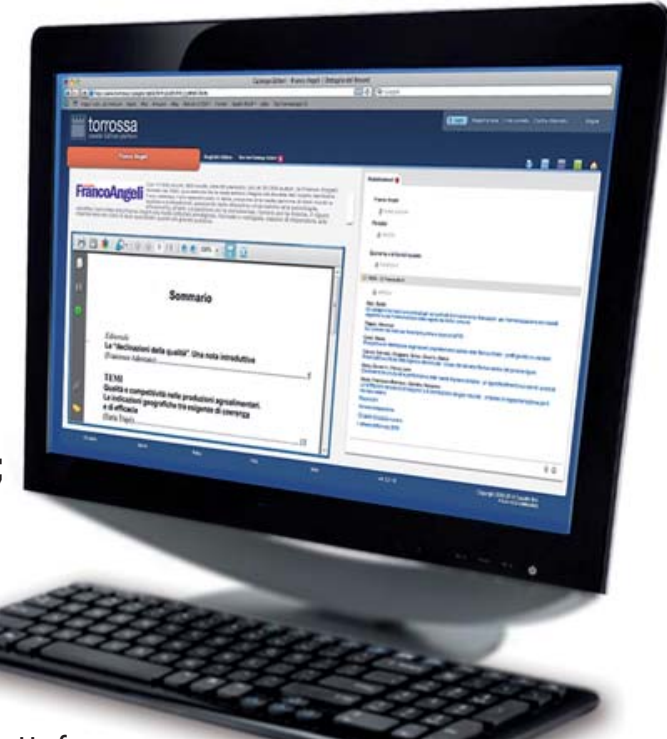

La fruizione di tutti i contenuti avviene tramite la piattaforma

\section{Torrossa - Casalini Full Text Platform}
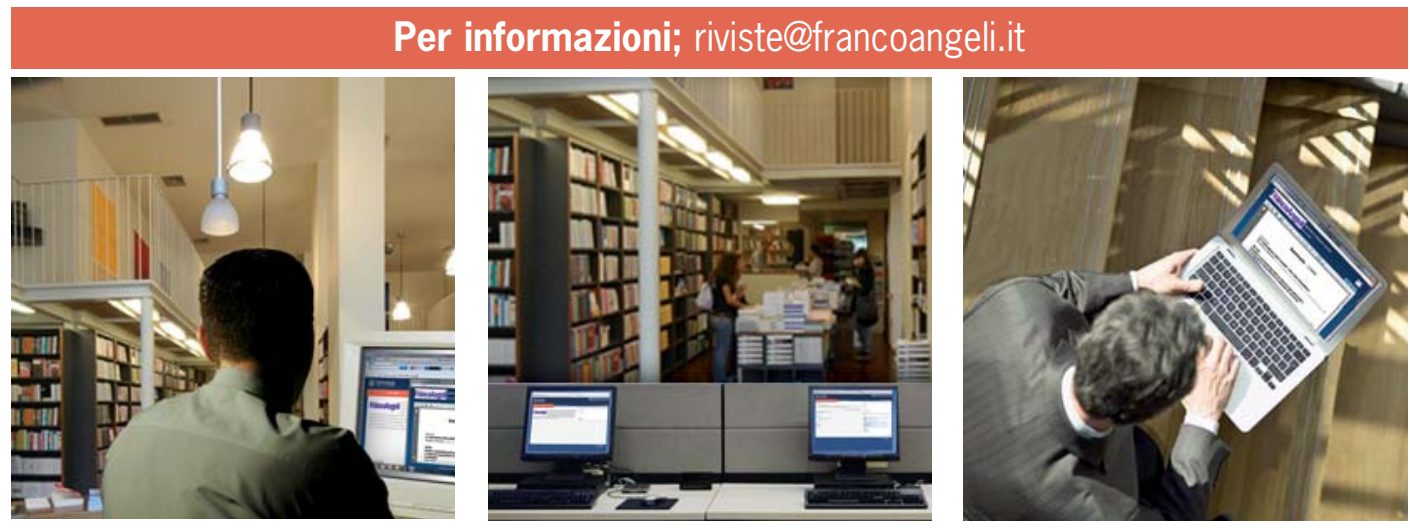

Copyright (C) FrancoAngeli

N.B: Copia ad uso personale. È vietata la riproduzione (totale o parziale) dell'opera con qualsiasi mezzo effettuata e la sua messa a disposizione di terzi, sia in forma gratuita sia a pagamento. 